\title{
A Stochastic Approximation ECM Algorithm for Misspecified Multivariate Probit Models
}

by

\author{
Abel Dasylva \\ A Thesis submitted to \\ the Faculty of Graduate Studies and Research \\ in partial fulfilment of \\ the requirements for the degree of \\ Master of Science \\ Ottawa-Carleton School of Mathematics and Statistics \\ Department of Mathematics and Statistics \\ Carleton University \\ Ottawa, Ontario, Canada \\ December 2009
}

Copyright (C)

2009 - Abel Dasylva 
Library and Archives

Canada

Published Heritage

Branch

395 Wellington Street Ottawa ON K1A ON4 Canada
Bibliotheque et

Archives Canada

Direction du

Patrimoine de l'édition

395, rue Wellington Ottawa ON K1A ON4

Canada
Your file Votre référence

ISBN: 978-0-494-64438-6

Our file Notre référence

ISBN: 978-0-494-64438-6
NOTICE:

The author has granted a nonexclusive license allowing Library and Archives Canada to reproduce, publish, archive, preserve, conserve, communicate to the public by telecommunication or on the Internet, loan, distribute and sell theses worldwide, for commercial or noncommercial purposes, in microform, paper, electronic and/or any other formats.

The author retains copyright ownership and moral rights in this thesis. Neither the thesis nor substantial extracts from it may be printed or otherwise reproduced without the author's permission.
AVIS:

L'auteur a accordé une licence non exclusive permettant à la Bibliothèque et Archives Canada de reproduire, publier, archiver, sauvegarder, conserver, transmettre au public par télécommunication ou par l'Internet, prêter, distribuer et vendre des thèses partout dans le monde, à des fins commerciales ou autres, sur support microforme, papier, électronique et/ou autres formats.

L'auteur conserve la propriété du droit d'auteur et des droits moraux qui protège cette thèse. $\mathrm{Ni}$ la thèse ni des extraits substantiels de celle-ci ne doivent être imprimés ou autrement reproduits sans son autorisation.
In compliance with the Canadian Privacy Act some supporting forms may have been removed from this thesis.

While these forms may be included in the document page count, their removal does not represent any loss of content from the thesis.
Conformément à la loi canadienne sur la protection de la vie privée, quelques formulaires secondaires ont étè enlevés de cette thèse.

Bien que ces formulaires aient inclus dans la pagination, il n'y aura aucun contenu manquant. 
The undersigned recommend to the Faculty of Graduate Studies and Research acceptance of the Thesis

\title{
A Stochastic Approximation ECM Algorithm for Misspecified Multivariate Probit Models
}

\author{
Submitted by Abel Dasylva \\ in partial fulfilment of the requirements for the degree of \\ Master of Science
}

S. Sinha, Supervisor

D. Cheung, Department Chair

Carleton University

2009 


\section{Abstract}

Multivariate probit models are used to study clustered data with binary and continuous responses. In these models, random effects are often assumed to follow a normal distribution. However this assumption is difficult to verify in practice, resulting in potential misspecification. Misspecification may be a serious problem for maximum likelihood fitting, which is commonly used in the estimation of generalized linear mixed models. A possible solution is to model random effects by a normal mixture, in the so-called heterogeneity model, and to apply an EM algorithm for estimating fixed and random effects parameters. Similar algorithms were proposed in previous studies, which suffered from being slow to converge.

In this work, it is shown that misspecification has a severe impact on ML estimates in correlated probit models with continuous and binary responses, when clusters belong to two latent classes that significantly differ in their random effects.

Also a Stochastic Approximation ECM algorithm is proposed for fitting the heterogeneity model, and its performance is studied through simulations. 


\section{Acknowledgments}

I would like to thank Professor Sinha for inspiring this work. I am greatly indebted to him for his supervision and support. 


\section{Table of Contents}

$\begin{array}{ll}\text { Abstract } & \text { iii }\end{array}$

Acknowledgments $\quad$ iv

Table of Contents $\quad$ v

List of Tables $\quad$ viii

Abbreviations $\quad$ ix

1 Introduction $\quad 1$

1.1 Statement of the problem .................... 8

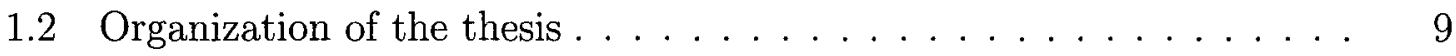

2 Generalized Linear Models $\quad 10$

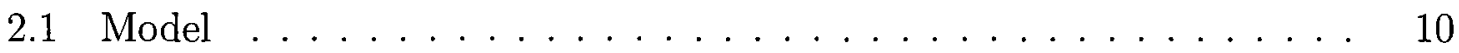

2.2 Estimation . . . . . . . . . . . . . . . . 11

2.2.1 Maximum likelihood .................. 11

2.2.2 Maximum Quasi-Likelihood (MQL) . . . . . . . . . . 13

3 Generalized Linear Mixed Models $\quad 14$

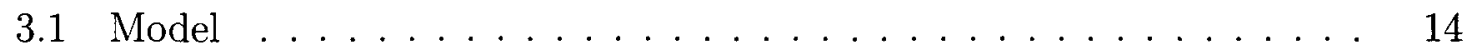

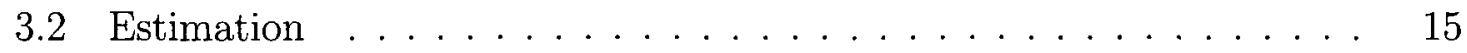


3.2.1 ML estimation . . . . . . . . . . . . . . . . 15

3.2.2 ML estimation as a missing data problem $\ldots \ldots \ldots \ldots . . \ldots 17$

3.2.3 Generalized Estimating Equations . . . . . . . . . . . . . . 19

3.2.4 Penalized Quasi Likelihood (PQL) . . . . . . . . . . 20

3.3 The EM algorithm and its variants $\ldots \ldots \ldots \ldots \ldots \ldots \ldots$

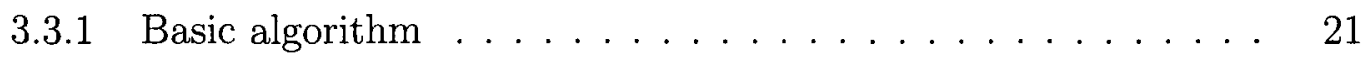

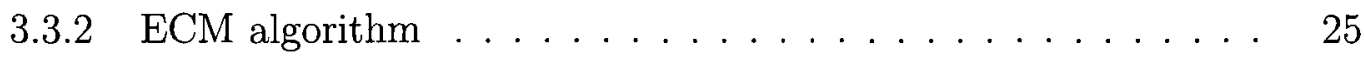

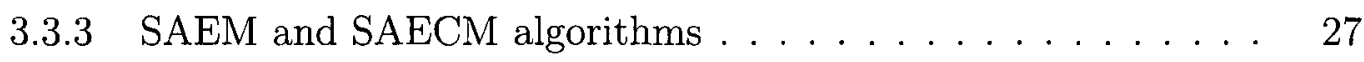

4 Multivariate probit models $\quad 36$

4.1 Random effects with a normal distribution $\ldots \ldots \ldots \ldots$

4.1 .1 Complete data . . . . . . . . . . . . . . . 39

4.1 .2 Observed data . . . . . . . . . . . . . . . 41

4.2 Random effects according to a normal mixture . . . . . . . . 41

4.2 .1 Complete data . . . . . . . . . . . . . . . . . 42

4.2 .2 Observed data $\ldots \ldots \ldots \ldots \ldots \ldots \ldots \ldots \ldots$

5 SAECM algorithms 46

5.1 Random effects with a normal distribution $\ldots \ldots \ldots \ldots$. . . . 47

5.1 .1 E step . . . . . . . . . . . . . . 47

$5.1 .2 \quad \mathrm{M}$ step $\ldots \ldots \ldots \ldots \ldots \ldots \ldots \ldots \ldots \ldots$

5.1 .3 Gibbs sampler . . . . . . . . . . . . . . . 49

5.2 Random effects following a normal mixture $\ldots \ldots \ldots \ldots \ldots 1$

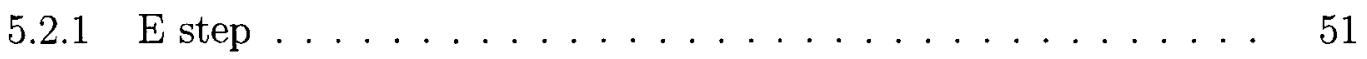

$5.2 .2 \quad$ M step . . . . . . . . . . . . . . 53

5.2 .3 Gibbs sampler $\ldots \ldots \ldots \ldots \ldots \ldots \ldots \ldots \ldots$ 
$\begin{array}{llr}6 & \text { Simulations } & \mathbf{5 7}\end{array}$

6.1 Random effects with a normal distribution . . . . . . . . . . 59

6.2 Random effects following a normal mixture . . . . . . . . . . . 61

6.2 .1 Misspecified model ................. 62

6.2 .2 Correct model ..................... 62

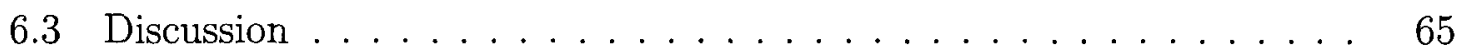

7 Conclusion $\quad 66$

$\begin{array}{ll}\text { List of References } & 68\end{array}$

$\begin{array}{ll}\text { Appendix A Matrix theory } & 71\end{array}$

A.1 Partitioned and patterned matrices . . . . . . . . . . 71

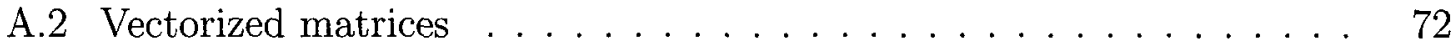

A.3 Differentiation . . . . . . . . . . . . . . 72

$\begin{array}{ll}\text { Appendix B Complete data information matrix } & 75\end{array}$

$\begin{array}{ll}\text { Appendix C R code } & 83\end{array}$ 


\section{List of Tables}

6.1 Parameters values when the random effects have a normal distribution. 60

6.2 MLE statistics for fixed effects and random effects parameters, in a correctly specified model with normal random effects, $K=100$ and $n=2,4 \ldots \ldots \ldots \ldots \ldots \ldots \ldots \ldots \ldots \ldots \ldots \ldots \ldots \ldots \ldots \ldots \ldots$

6.3 MLE statistics of fixed effets and random effects, in a misspecified model with random effects following a two-components normal mixture but fitted assuming normal random effects, with $K=100$ and $n=2,4$.

6.4 MLE statistics of fixed effets and random effects, in a correctly specified model with random effects following a two-components normal mixture, with $K=100$ and $n=2,4 \ldots \ldots \ldots \ldots$

6.5 MLE statistics for mixture components, in a correctly specified model with random effects following a two-components normal mixture, with $K=100$ and $n=2,4 \ldots \ldots \ldots \ldots \ldots \ldots \ldots$ 


\section{Abbreviations}

ECM Expectation Conditional Maximization

EM Expectation Maximization

GEE Generalized Estimating Equation

GLM Generalized Linear Model

GLMM Generalized Linear Mixed Model

ML Maximum Likelihood

MLE Maximum Likelihood Estimate

MQL Maximum Quasi-Likelihood

SA Stochastic Approximation

SAECM Stochastic Approximation Expectation Conditional Maximization

SAEM Stochastic Approximation Expectation Maximization 


\section{Chapter 1}

\section{Introduction}

Generalized Linear Mixed Models (GLMMs) provide convenient models for experiments with correlated or repeated measurements. The features of these models are as follows [1]. Each cluster $i=1, \ldots, K$ is characterized by an unknown vector of random effects $\mathbf{U}_{i}$, which are assumed to be independent and identically distributed across all the clusters. The random effect $\mathbf{U}_{i}$ affects all the $n_{i}$ observations $\mathbf{Y}_{i 1}, \ldots, \mathbf{Y}_{i n_{i}}$ within cluster $i$, thereby generating the intra-cluster correlation. Conditional on $\mathrm{U}_{i}$, the responses $\mathbf{Y}_{i 1}, \ldots, \mathbf{Y}_{i n_{i}}$ are assumed to be independent according to some distribution in the exponential family. The model may also be expressed by a relationship between the conditional mean of the response given the random effects, and the covariates for the fixed and random effects:

$$
E\left[\mathbf{Y}_{i j} \mid \mathbf{U}_{i}=\mathbf{u}_{i}\right]=\boldsymbol{\mu}_{i}, \quad g\left(\boldsymbol{\mu}_{i}\right)=\mathbf{x}_{i}^{\top} \boldsymbol{\beta}+\mathbf{z}_{i}^{\top} \mathbf{u}_{i}
$$

where $g$ is a link function, $\boldsymbol{\beta}$ is a vector of regression coefficients, $\mathbf{X}_{i}$ and $\mathbf{Z}_{i}$ are known vectors. The goal is to make inferences about the regression coefficients $\boldsymbol{\beta}$ and the parameters of the random effects distribution.

Although GLMMs are used for analyzing clustered data, fixed effects parameters may be estimated using marginal models that assume independent Generalized Linear 
Models for the different responses, i.e.

$$
E\left[\mathbf{Y}_{i j}\right]=\boldsymbol{\mu}_{i}, \quad g\left(\boldsymbol{\mu}_{i}\right)=\mathbf{x}_{i}^{\top} \boldsymbol{\beta}
$$

Marginal models treat random effects parameters as nuisance parameters. The widely used Generalized Estimating Equations (GEEs) provide consistent estimators of regression coefficients, even when the covariance matrix is poorly characterized [2]. Also the obtained estimates are robust to misspecified random effects. They may be interpreted as population-averaged estimators. However marginal models have many limitations and they are not always appropriate [3]. For example, in longitudinal clinical studies, it is essential to model the patient-treatment interaction over time, and to account for the correlation between the repeated measurements for each patient. Marginal models are also less general than conditional models, since a marginal model may be derived from each conditional model while the converse is not true $[3,4]$. In fact certain marginal models may altogether preclude the existence of any underlying conditional model [3].

In conditional models, parameters are usually estimated based on the maximum likelihood. Maximum likelihood estimation is a well established procedure. Under mild regularity conditions, it produces consistent and efficient estimators. However it requires a perfect knowledge of the parametric distribution from which the data is drawn. In practice, even in the simplest GLMMs, i.e. Linear Mixed Models (LMMs), it is difficult to verify assumptions about the distribution of random effects based on their empirical Bayes estimates, due to shrinkage [5].

When models are misspecified, the standard asymptotic theory of ML estimation no longer applies. This has many negative consequences. For example ML estimates may no longer be consistent or efficient. Instead, MLEs provide consistent estimators 
of the paramaters values that minimize the Kullback-Leibler distance between the assumed family of distributions, and the actual distribution of the data. H. White has provided a rigorous proof of this result along with the following heuristic argument [6]. Assume iid observations $\mathbf{Y}_{i}$ according to a density $g$, and a family of densities $f(., \boldsymbol{\theta})$ not containing the actual density $g$, and it is inaccurately assumed that the data are sampled from $f(., \boldsymbol{\theta})$. The MLE $\widehat{\boldsymbol{\theta}}$ minimizes $-\sum_{i=1}^{m} \log f\left(\mathbf{y}_{i}, \boldsymbol{\theta}\right)$, which in the large sample case (by the law of large numbers) may be approximated as follows:

$$
-\sum_{i=1}^{m} \log f\left(\mathbf{y}_{i}, \boldsymbol{\theta}\right) \approx-m E\left[\log f\left(\mathbf{Y}_{1}, \boldsymbol{\theta}\right)\right]=-m \int \log f(\mathbf{y}, \boldsymbol{\theta}) g(\mathbf{y}) d \mathbf{y}
$$

Therefore $\widehat{\boldsymbol{\theta}}$ provides a good estimator of $\boldsymbol{\theta}^{*}$ that minimizes the Kullback-Leibler distance defined as $D(g \| f(., \boldsymbol{\theta})):=\int \log f(\mathbf{y}, \boldsymbol{\theta}) g(\mathbf{y}) d \mathbf{y}-H(g)$, where $H$ is the entropy of $g$.

In the context of misspecification, misleading results are obtained from tests that rely on the standard asymptotic theory of MLEs, e.g. the Likelihood Ratio Test.

Many authors have discussed the impact of misspecified random effects on ML estimation in GLMMs. Other forms of misspecification have been studied as well. However our focus is on misspecification of the distribution of random effects, which will be simply referred to as misspecification. In the past, the consensus was that misspecifying random effects had little impact on fixed effects parameters, but potentially a large impact on random effects parameters [7]. That opinion was motivated by studies on specific GLMMs, and it has justified the assumption of normal random effects in many cases. For example M. Neuhaus et al. have studied mixed effects logistic models [8-10]. They showed that misspecification caused small bias in the MLEs of fixed effects parameters, and it did not significantly affect standard error estimates. In the same models, Heagerty and Kurland showed that marginal regression parameters are even less sensitive to misspecification [11]. Also, for linear mixed 
models Verbeke and Lesaffre have proved that the MLEs of fixed effects parameters remained consistent with an asymptotic normal distribution, even when the random effects did not have a normal distribution [12].

To obtain better estimates of random effects, Verbeke and Lesaffre have proposed the heterogeneity model, an LMM where random effects follow an homoschedastic normal mixture [5]. The heterogeneity model provides a flexible way to fit different distributions of random effects, and to account for the omission of influential categorical variates in the model. EM algorithms [5] and modified Newton-Raphson algorithms [13] were used to compute ML estimates, and the number of mixture components were estimated using likelihood ratio tests, goodness of fit [5] and Akaike's Information Criteria (AIC) [13].

In recent studies, Agresti et al. [14] and S. Litiere et al. have shown that misspecified random effects may significantly impact fixed effect parameters $[15,16]$. Agresti et al. observed that misspecification dramatically reduced the efficiency of variance estimators, especially when the random effects were generated according to a discrete two-point distribution, and fitted according to the normal assumption [14]. S. Litiere et al. reported that misspecifiaction affected estimators of fixed-effect parameters, and type I error for tests of their significance, especially when the data could be modeled by a latent class model with a large variance component $[15,16]$. In a latent class model, subjects (clusters) belong to two or more classes with widely different characteristics of their random effects. However, the class of each subject is not observed.

In their work, Agresti et al. presented three examples where misspecified random effects affected the estimation of fixed and random effects parameters, in the case of a GLMM with a logistic link and univariate responses. The GLMM was given by the 
following equation:

$$
Y_{i j} \mid U_{i}=u_{i} \sim \operatorname{Bernoulli}\left(\mu_{i}\right), \quad \operatorname{logit}\left(\mu_{i}\right)=\beta+u_{i}, i=1, \ldots, K
$$

Data were generated with random-effects following different distributions, including normal, uniform, exponential, binary, and degenarate at one point. Then estimates of $\beta$ and $\sigma^{2}=\operatorname{var}\left(u_{i}\right)$ were obtained, using a nonparametric algorithm [17], and using different parametric assumptions for the distribution of random effects, e.g. a normal distribution. The nonparametric algorithm fitted the model based on a discrete random effects distribution, with a given number of mass points at unspecified locations. Agresti et al. have observed that misspecification had the greatest impact, in the latent class model, where the random effects had a symmetric two-point discrete distribution and they were fitted according to the normal assumption. The impact of the misspecification was mostly felt on the estimated variance, and it increased with the number of clusters, the cluster size and the size of the variance component, up to an order of magnitude. However, in most cases the estimated regression component was not sgnificantly affected except in one case. Motivated by those results, Agresti et al. suggested practical guidelines for fitting models involving random effects. A preliminary analysis should determine whether the data is best modeled by a latent class model, where subjects (clusters) belong to two or more classes with different characteristics [18]. Also, models should be fitted using both a parametric method and a nonparametric method. Results of such studies should be interpreted with caution when significant differences occur.

S. Litiere et al. gave another example of logistic GLMM where misspecified random effects had a large impact on Type I error [15]. The GLMM was used in a longitudinal study to measure the effect of Risperidone, an anti-psychotic agent used in the treatment of chronic schizophrenia. One hundred and twenty-eight patients were included 
in the clinical trial, and randomly assigned either to a treatment group or a control group. In the treatment group, each patient was given a daily dose of risperidone from 4 to $6 \mathrm{mg}$, for eight weeks. The mental condition of each patient was assessed at the begining of the study and after $1,2,4,6$, and 8 weeks, according to the Clinical Global Impression (CGI), a 7-grade subjective measure. The CGI scale was converted to a binary variable $y_{i j}$ by setting $y_{i j}=1$ if patient $i$ was classified as normal to mildly ill on the $j$-th measurement, and otherwise setting $y_{i j}=0$. The resulting data were fitted using the following logistic model and assuming normal random effects, where $Z_{i}=0$ for patients in the control group and $Z_{i}=1$ for patients in the treatment group.

$$
\operatorname{logit}\left(\mu_{i j}\right)=\beta_{0}+\beta_{1} Z_{i}+\beta_{2} t_{j}+u_{i}
$$

The estimated variance was quite large (above 20), a distinctive feature of that work. Simulation results have shown that for such large random effects, any misspecification potentially had a dramatic impact on type I error for tests of significance. The type I error for testing whether $\beta_{0}=0$ was significantly increased by misspecified random effects even when their variance was small (e.g. $\sigma^{2}=1$ ). The impact on the type I error for testing $\beta_{1}=0$ was affected to a lesser extent, and it was not always increased by misspecification In both cases, the impact of misspecification became more pronounced when the number of patients and the variance of random effects increased. However for fixed effects that were not included in the random effects structure (e.g. in randomized clinical trials), S. Litiere et al. showed that their tests of significance were not affected by misspecification.

In the frequentist perspective, different solutions have been suggested to mitigate misspecification in general GLMMs, including a fully nonparametric approach suggested by Aitkin [17], and the heterogeneity model suggested by Verbeke and Lesaffre [5]. 
The nonparametric method models the random effects according to a discrete distribution with a given number of mass points at unspecified locations. In large models with many random effects, nonparametric solutions are computer intensive and their parameters are not always identifiable. In the heterogeneity model, random effects are modeled by a mixture of normal distributions, a choice that offers many advantages. First mixture parameters may be estimated using EM algorithms as in missing data poblems [19]. Second in the specific case of multivariate probit models, the complete data ML equations correspond to an LMM. Therefore they have a simple expression. However fitting the heterogeneity model may be numerically challenging, especially when the number of mixture components is large.

S. Litiere et al. have used the heterogeneity model to analyze the data from the clinical trial on risperidone, described above [16]. To avoid any overparametrization, they modified the logistic model as

$$
\operatorname{logit}\left(\mu_{i j}\right)=\beta_{1} Z_{i}+\beta_{2} t_{j}+u_{i}
$$

where $u_{i} \sim \pi_{1} N\left(\mu_{1}, d\right)+\left(1-\pi_{1}\right) N\left(\mu_{2}, d\right)$ for a two-component mixture (where $\left.\beta_{0}=\pi_{1} \mu_{1}+\pi_{2} \mu_{2}\right)$, and $u_{i} \sim \pi_{1} N\left(\mu_{1}, d\right)+\pi_{2} N\left(\mu_{2}, d\right)+\left(1-\pi_{1}-\pi_{2}\right) N\left(\mu_{3}, d\right)$ for a three conponent mixture (where $\beta_{0}=\pi_{1} \mu_{1}+\pi_{2} \mu_{2}+\pi_{3} \mu_{3}$ ). In simulations, the heterogeneity model generally provided better estimates than those obtained by assuming normal random effects. However, the convergence of the proposed EM algorithm was slow and the resulting algorithm was not practical.

In this work, a new algorithm is proposed for fitting the heterogeneity model. It is based on an extension of a Stochastic Approximation Expected Conditional Maximization (SAECM) algorithm originally proposed by R. Gueorguieva and A. Agresti 
[20]. The original algorithm has provided an effective way of estimating the parameters of a multivariate probit models with continuous and discrete responses, and with normal random effects. Its main advantage has been its faster rate of convergence compared to a conventional ECM algorithm proposed by Chan et al. [21]. The use of stochastic approximations has many advantages [22]. First it replaces computer intensive Expectation steps with simpler stochastic approximations steps. Second it makes more effective uses of random samples generated during the computation. $\mathrm{R}$. Gueorguieva and A. Agresti have compared their algorithm to a conventional ECM procedure and shown that the former converged in a larger number of iterations but in less time. They also gave an example where the SAECM procedure converged while convergence was hard to obtain with the ECM algorithm of Chan et al. [21]. The newly proposed algorithm is an extension of the SAECM procedure to accomodate random effects distributed according to a normal mixture. Simulations show that this extension is effective in reducing the MLE bias resulting from misspecified random effects, including in latent class models with large random effects and severe polarization between classes. The same results also show that contrary to the opinion expressed in [20] misspecification may significantly impact ML estimates in correlated probit models with continuous and binary responses.

\subsection{Statement of the problem}

The heterogeneity model has been proposed to reduce the impact of misspecified random effects in multivariate probit models. However its application has been limited by the lack of an effective ML fitting procedure. A new SAECM procedure is proposed for this purpose. 


\subsection{Organization of the thesis}

The remaining chapters are organized as follows. Chapters 2 and 3 provide an overview of Generalized Linear Models (GLMs) and Generalized Linear Mixed Models (GLMMs), including algorithms to estimate the parameters in such models. More emphasis is placed on the description of the EM algorithm and its variants, including the ECM, SAEM and SAECM algorithms. Chapter 4 presents the heterogeneity model and derives its ML equations including the score and observed information matrix, computed according to Louis's missing information principle [23]. Chapter 5 describes the proposed SAECM algorithm for the heterogeneity model. Chapter 6 presents simulation results. Chapter 7 presents the conclusion of this thesis. 


\section{Chapter 2}

\section{Generalized Linear Models}

GLMMs are best described as extensions of GLMs and by reference to them. This chapter provides a brief overview of GLMs to serve as a basis for the next chapter on GLMMs. The material is drawn from [1].

\section{$2.1 \quad$ Model}

Generalized Linear Models are an extension of linear models for the analysis of independent observations $y_{1}, \ldots, y_{n}$ that do not necessarily follow a normal distribution. The model is characterized by the following components:

- The distributions of individual observations: assumed to be in the exponential family and of the (canonical) form

$$
f_{Y_{i}}\left(y_{i}\right)=\exp \left(\frac{y_{i} \gamma_{i}-b\left(\gamma_{i}\right)}{\tau^{2}}-c\left(y_{i}, \tau\right)\right) .
$$

- The link function $g$ that relates the mean of individual observations to a linear predictor

$$
E\left[y_{i}\right]=\mu_{i}, \quad g\left(\mu_{i}\right)=\mathbf{x}_{i}^{\top} \boldsymbol{\beta},
$$


where $\mathbf{x}_{i}$ is a vector of explanatory variables and $\boldsymbol{\beta}$ is a vector of regression coefficients.

- A linear predictor of the form $\mathbf{x}_{i}^{\top} \boldsymbol{\beta}$.

Examples of link functions include the folllowing:

- The identity link: $g(\mu)=\mu$

- The log-link: $g(\mu)=\log \mu$

- The log-log link: $g(\mu)=\log (-\log (1-\mu))$

- The logistic link: $g(\mu)=\log \left(\frac{\mu}{1-\mu}\right)$

- The probit link: $g(\mu)=\Phi^{-1}(\mu)$

Linear models correspond to GLMs with normal observations and the identity link.

\subsection{Estimation}

The regression coefficients are the main interest of the estimation procedure.

\subsubsection{Maximum likelihood}

The ML estimators are obtained by maximizing the log-likelihood which has the following expression:

$$
\log L(\boldsymbol{\beta} ; \mathbf{y})=\sum_{i=1}^{n} \frac{y_{i} \gamma_{i}-b\left(\gamma_{i}\right)}{\tau^{2}}-\sum_{i=1}^{n} c\left(y_{i}, \tau\right)
$$

It can be shown that

$$
\frac{\partial \log L(\boldsymbol{\beta} ; \mathbf{y})}{\partial \boldsymbol{\beta}}=\frac{1}{\tau^{2}} \mathbf{X}^{\top} \mathbf{W} \boldsymbol{\Delta}(\mathbf{y}-\boldsymbol{\mu}),
$$


where

$$
\begin{aligned}
& \mathbf{W}=\left[\begin{array}{ccc}
w_{1} & & (0) \\
& \ddots & \\
(0) & & w_{n}
\end{array}\right], \boldsymbol{\Delta}=\left[\begin{array}{ccc}
g_{\mu}\left(\mu_{1}\right) & & (0) \\
& \ddots & \\
& & \\
(0) & & g_{\mu}\left(\mu_{n}\right)
\end{array}\right], \mathbf{y}=\left[\begin{array}{c}
y_{1} \\
\vdots \\
y_{n}
\end{array}\right], \boldsymbol{\mu}=\left[\begin{array}{c}
\mu_{1} \\
\vdots \\
\mu_{n}
\end{array}\right], \\
& w_{i}=\frac{1}{v\left(\mu_{i}\right) g_{\mu}\left(\mu_{i}\right)^{2}}, \quad v\left(\mu_{i}\right)=\frac{\partial^{2} b\left(\gamma_{i}\right)}{\partial \gamma_{i}^{2}}, \quad g_{\mu}\left(\mu_{i}\right)=\frac{\partial g\left(\mu_{i}\right)}{\partial \mu_{i}}
\end{aligned}
$$

and

$$
E\left[y_{i}\right]=\mu_{i}=\frac{\partial b\left(\gamma_{i}\right)}{\partial \gamma_{i}}, \quad \operatorname{var}\left(y_{i}\right)=\tau^{2} \frac{\partial^{2} b\left(\gamma_{i}\right)}{\partial \gamma_{i}^{2}}
$$

Also

$$
\frac{\partial^{2} \log L(\boldsymbol{\beta} ; \mathbf{y})}{\partial \boldsymbol{\beta} \partial \boldsymbol{\beta}^{\top}}=-\frac{1}{\tau^{2}} \mathbf{X}^{\top} \mathbf{W} \boldsymbol{\Delta} \frac{\partial \boldsymbol{\mu}}{\partial \boldsymbol{\beta}^{\top}}+\frac{1}{\tau^{2}} \mathbf{X}^{\top} \frac{\partial \mathbf{W} \boldsymbol{\Delta}}{\partial \boldsymbol{\beta}^{\top}}(\mathbf{y}-\boldsymbol{\mu})
$$

The MLE may be computed iteratively using the Newton-Raphson technique as follows:

$$
\boldsymbol{\beta}^{(m+1)}=\boldsymbol{\beta}^{(m)}+\left(\mathbf{X}^{\top} \mathbf{W X}\right)^{-1} \mathbf{X}^{\top} \mathbf{W} \boldsymbol{\Delta}(\mathbf{y}-\boldsymbol{\mu})
$$

The same equation may be written as a function of the working variate $\mathbf{t}$ defined as

$$
\mathbf{t}^{(m)}:=\mathbf{X} \boldsymbol{\beta}^{(m)}+\boldsymbol{\Delta}(\mathbf{y}-\boldsymbol{\mu})
$$

Then

$$
\boldsymbol{\beta}^{(m+1)}=\left(\mathbf{X}^{\top} \mathbf{W} \mathbf{X}\right)^{-1} \mathbf{X}^{\top} \mathbf{W} \mathbf{t}^{(m)}
$$

The large sample variance of the MLE $\hat{\boldsymbol{\beta}}$ is obtained as

$$
\operatorname{var}_{\infty}(\hat{\boldsymbol{\beta}})=\tau^{2}\left(\mathbf{X}^{\top} \mathbf{W X}\right)^{-1}
$$




\subsubsection{Maximum Quasi-Likelihood (MQL)}

The method of maximum quasi-likelihood is used when the actual distribution of the observations is not known, except for the mean-variance relationship. This knowledge is specified in the form of a function $v\left(\mu_{i}\right)$ such that the variance of $y_{i}$ is proportional to $v\left(\mu_{i}\right)$ where $E\left[y_{i}\right]=\mu_{i}$. The quasi-likelihood is defined as $\sum_{i=1}^{n} Q_{i}\left(\mu_{i} ; y_{i}\right)$, where

$$
Q_{i}\left(\mu_{i} ; y_{i}\right)=\int_{y_{i}}^{\mu_{i}} \frac{y_{i}-t}{\tau^{2} v(t)} d t
$$

The MQL estimator of $\boldsymbol{\beta}$ solves the MQL equation

$$
\frac{\partial}{\partial \beta} \sum_{i=1}^{n} Q_{i}\left(\mu_{i} ; y_{i}\right)=\mathbf{0}
$$

which is equivalent to the ML estimating equation under the exponential family (Equation (2.3)). Under appropriate regularity conditions, the MQL estimator $\hat{\boldsymbol{\beta}}$ is asymptotically normal with mean $\boldsymbol{\beta}$ and covariance matrix $\tau^{2}\left(\mathrm{X}^{\top} \mathbf{W X}\right)^{-1}$. 


\section{Chapter 3}

\section{Generalized Linear Mixed Models}

The material of Sections 3.1 and 3.2 is mostly drawn from [1] with references to original articles where appropriate. The content of Section 3.3 is drawn from articles on the EM algorithm and its different variants: ECM, SAEM and SAECM.

\subsection{Model}

Generalized Linear Mixed Models are an extension of GLMs to model correlated or repeated observations. Observations are distributed across many clusters. Responses in the same cluster are correlated while responses across different clusters are assumed independent. Let $K$ denote the number of independent clusters, $n_{i}$ the number of (univariate) observations in cluster $i, \mathbf{u}_{i}$ the vector of zero-mean random effects and $y_{i j}$ the $j$-th observation in the cluster. Conditional on $\mathbf{u}_{1}, \ldots, \mathbf{u}_{K}$, the observations $y_{i j}$ are assumed to follow a GLM as described in the previous chapter. The conditional mean of $y_{i j}$ given $u_{i}$ has the form

$$
E\left[y_{i j} \mid \mathbf{u}_{i}\right]=\mu_{i}, \quad g\left(\mu_{i}\right)=\mathbf{x}_{i}^{\top} \boldsymbol{\beta}+\mathbf{z}_{i}^{\top} \mathbf{u}_{i}
$$


where $g$ is a known 1-1 link function, $\mathbf{x}_{i}$ and $\mathbf{z}_{i}$ are known design matrices respectively for the fixed and random effects, and $\boldsymbol{\beta}$ is a vector of regression coefficients. The random effects $\mathbf{u}_{1}, \ldots, \mathbf{u}_{K}$ are assumed iid, according to some density $f_{\mathbf{u}}$ in the exponential family.

A GLMM may include multivariate observations in each cluster. In this case the link function $g$ is also multivariate. Linear Mixed Models form an important subclass of GLMMs. They are specified as follows:

$$
E\left[\mathbf{Y}_{i j} \mid \mathbf{u}_{i}\right]=\mathbf{x}_{i}^{\top} \boldsymbol{\beta}+\mathbf{z}_{i}^{\top} \mathbf{u}_{i}
$$

where $\mathbf{Y}_{i j}$ is the $j$-th vector of responses for cluster $i$.

\subsection{Estimation}

In GLMMs, the goal is the estimation of the regression coefficients, and the parameters characterizing the distribution of random effects. To this end two approaches are mostly used including solutions based on Generalized Estimating Equations (GEEs) and solutions based on the maximum likelihood principle. Another approach based on the concept of conditional lilekihood is also described in [1].

\subsubsection{ML estimation}

The log-likelihood has a more complex expression compared to the GLM case:

$$
\log L(\boldsymbol{\beta}, \mathbf{D} ; \mathbf{y})=\sum_{i=1}^{K} \log \left(\int f_{\mathbf{u}}(\mathbf{u}) \prod_{j=1}^{n_{i}} \exp \left(\frac{y_{i j} \gamma_{i}(\mathbf{u})-b\left(\gamma_{i}(\mathbf{u})\right)}{\tau^{2}}-c\left(y_{i j}, \tau\right)\right) d \mathbf{u}\right)
$$


After some algebra, the score has the form

$$
\frac{\partial \log L(\boldsymbol{\beta}, \mathbf{D} ; \mathbf{y})}{\partial \boldsymbol{\beta}}=\mathbf{X}^{\top} E\left[\mathbf{W}^{*} \mid \mathbf{y}\right] \mathbf{y}-\mathbf{X}^{\top} E\left[\mathbf{W}^{*} \boldsymbol{\mu} \mid \mathbf{y}\right]
$$

where the expectation is taken with respect to the conditional distribution of $\mathbf{u}$ given $\mathbf{y}$ and

$$
\begin{aligned}
& \mathbf{X}=\left[\begin{array}{c}
\mathbf{x}_{1}^{\top} \otimes \mathbf{1}_{n_{1} \times 1} \\
\vdots \\
\mathbf{x}_{K}^{\top} \otimes \mathbf{1}_{n_{K} \times 1}
\end{array}\right], \mathbf{y}=\left[\begin{array}{c}
\mathbf{y}_{1} \\
\vdots \\
\mathbf{y}_{K}
\end{array}\right], \quad \mathbf{y}_{i}=\left[\begin{array}{c}
\mathbf{y}_{i 1} \\
\vdots \\
\mathbf{y}_{i n_{i}}
\end{array}\right], \boldsymbol{\mu}=\left[\begin{array}{c}
\mu_{1} \mathbf{1}_{n_{1} \times 1} \\
\vdots \\
\mu_{K} \mathbf{1}_{n_{K} \times 1}
\end{array}\right] \\
& \mathbf{W}^{*}=\left[\begin{array}{ccc}
\frac{1}{\tau^{2} v\left(\mu_{1}\right) g_{\mu}\left(\mu_{1}\right)} \mathbf{I}_{n_{1}} & (0) \\
& \ddots & \\
(0) & & \frac{1}{\tau^{2} v\left(\mu_{K}\right) g_{\mu}\left(\mu_{K}\right)} \mathbf{I}_{n_{K}}
\end{array}\right]
\end{aligned}
$$

Therefore $\boldsymbol{\beta}$ is estimated from the following ML equation

$$
\mathbf{X}^{\top} E\left[\mathbf{W}^{*} \mid \mathbf{y}\right] \mathbf{y}-\mathbf{X}^{\top} E\left[\mathbf{W}^{*} \boldsymbol{\mu} \mid \mathbf{y}\right]=\mathbf{0}
$$

Assuming that the random effects distribution also depends on some parameter $\mathbf{D}$, we have

$$
\frac{\partial \log L(\boldsymbol{\beta}, \mathbf{D} ; \mathbf{y})}{\partial \mathbf{D}}=E\left[\frac{\partial \log f_{\mathbf{u}}(\mathbf{u})}{\partial \mathbf{D}} \mid \mathbf{y}\right]
$$

The parameter $\mathbf{D}$ is estimated from the following $\mathrm{ML}$ equation

$$
E\left[\frac{\partial \log f_{\mathbf{u}}(\mathbf{u})}{\partial \mathrm{D}} \mid \mathbf{y}\right]=0
$$


Solving the ML equations numerically poses great challenges because of integration over the random effects. Numerically, high-dimensional integrals may be estimated through different methods including Gauss-Hermite quadrature (see [1]) and Expectation-Maximization algorithms.

The problem of ML estimation in GLMMs may also be discussed from the perspective of missing data.

\subsubsection{ML estimation as a missing data problem}

The major difficulty in GLMMs is that the observed data $\mathbf{Y}$ depends on the random effects $\boldsymbol{U}$ that are not directly observed, while the parameters $\boldsymbol{\psi}$ (including $\boldsymbol{\beta}$ ) to be estimated are associated with the distribution of $(\mathbf{Y}, \mathbf{U})$. Let $\mathbf{W}$ denote the complete data including both $\mathbf{Y}$ and $\mathbf{U}$ and any other relevant random variable. Let $f_{\mathbf{w}}(\mathbf{w} ; \boldsymbol{\psi})$ denote the density for the complete data, $f_{\mathbf{y}}(\mathbf{y} ; \boldsymbol{\psi})$ the density for the observed data, and $\mathscr{R}(\mathbf{y})$ the set of complete data for which the observed data is $\mathbf{y}$ with a nonzero probability. The score and information matrices for the observed data may be obtained from the conditional expectation of the score and information matrices for the complete data, given the observations. T. Louis has provided the following simple derivation of this result [23].

$$
f_{\mathbf{Y}}(\mathbf{y} ; \psi)=\int_{\mathscr{R}(\mathbf{y})} f_{\mathbf{w}}(\mathbf{w} ; \boldsymbol{\psi}) d \mathbf{w}
$$

In the specific case of GLMMs, it simply amounts to an integration with respect to the random effects as

$$
f_{\mathbf{Y}}(\mathbf{y} ; \boldsymbol{\psi})=\int_{\mathscr{R}(\mathbf{y})} f_{\mathbf{y}, \mathbf{u}}(\mathbf{y}, \mathbf{u} ; \boldsymbol{\psi}) d \mathbf{u}
$$


Let $\log L_{c}(\boldsymbol{\psi} ; \mathbf{w})$ denote the $\log$-likelihood for the compelete data, and $\log L(\boldsymbol{\psi} ; \mathbf{y})$ the log-likelihood for the observed data.

$$
\begin{aligned}
\frac{\partial \log L(\boldsymbol{\psi} ; \mathbf{y})}{\partial \boldsymbol{\psi}} & =\left[\int_{\mathscr{R}(\mathbf{y})} f_{\mathbf{w}}(\mathbf{w} ; \boldsymbol{\psi}) d \mathbf{w}\right]^{-1} \int_{\mathscr{R}(\mathbf{y})} \frac{\partial f_{\mathbf{w}}(\mathbf{w} ; \boldsymbol{\psi})}{\partial \boldsymbol{\psi}} d \mathbf{w} \\
& =\left[\int_{\mathscr{R}(\mathbf{y})} f_{\mathbf{w}}(\mathbf{w} ; \boldsymbol{\psi}) d \mathbf{w}\right]^{-1} \int_{\mathscr{R}(\mathbf{y})} \frac{\partial \log L_{c}(\boldsymbol{\psi} ; \mathbf{w})}{\partial \boldsymbol{\psi}} f_{\mathbf{w}}(\mathbf{w} ; \boldsymbol{\psi}) d \mathbf{w} \\
& =E\left[\frac{\partial \log L_{c}(\boldsymbol{\psi} ; \mathbf{w})}{\partial \boldsymbol{\psi}} \mid \mathbf{Y}=\mathbf{y}\right]
\end{aligned}
$$

The observed information matrix is obtained as follows:

$$
\begin{aligned}
\frac{\partial^{2} \log L(\boldsymbol{\psi} ; \mathbf{y})}{\partial \boldsymbol{\psi} \partial \boldsymbol{\psi}^{\top}}= & {\left[\int_{\mathscr{R}(\mathbf{y})} f_{\mathbf{w}}(\mathbf{w} ; \boldsymbol{\psi}) d \mathbf{w}\right]^{-1} \int_{\mathscr{R}(\mathbf{y})} \frac{\partial^{2} f_{\mathbf{w}}(\mathbf{w} ; \boldsymbol{\psi})}{\partial \boldsymbol{\psi} \partial \boldsymbol{\psi}^{\top}} d \mathbf{w}-} \\
& E\left[\frac{\partial \log L_{c}(\boldsymbol{\psi} ; \mathbf{w})}{\partial \boldsymbol{\psi}} \mid \mathbf{Y}=\mathbf{y}\right] E\left[\frac{\partial \log L_{c}(\boldsymbol{\psi} ; \mathbf{w})}{\partial \boldsymbol{\psi}} \mid \mathbf{Y}=\mathbf{y}\right]^{\top}
\end{aligned}
$$

However

$$
\begin{aligned}
& {\left[\int_{\mathscr{R}(\mathbf{y})} f_{\mathbf{w}}(\mathbf{w} ; \boldsymbol{\psi}) d \mathbf{w}\right]^{-1} \int_{\mathscr{R}(\mathbf{y})} \frac{\partial^{2} f_{\mathbf{w}}(\mathbf{w} ; \boldsymbol{\psi})}{\partial \boldsymbol{\psi} \partial \boldsymbol{\psi}^{\top}} d \mathbf{w}=} \\
& {\left[\int_{\mathscr{R}(\mathbf{y})} f_{\mathbf{w}}(\mathbf{w} ; \boldsymbol{\psi}) d \mathbf{w}\right]^{-1} \int_{\mathscr{R}(\mathbf{y})} \frac{1}{f_{\mathbf{w}}(\mathbf{w} ; \boldsymbol{\psi})^{2}}\left(f_{\mathbf{w}}(\mathbf{w} ; \boldsymbol{\psi}) \frac{\partial^{2} f_{\mathbf{w}}(\mathbf{w} ; \boldsymbol{\psi})}{\partial \boldsymbol{\psi} \partial \boldsymbol{\psi}^{\top}}-\right.} \\
& \left.\left\{\frac{\partial f_{\mathbf{w}}(\mathbf{w} ; \boldsymbol{\psi})}{\partial \boldsymbol{\psi}}\right\}\left\{\frac{\partial f_{\mathbf{w}}(\mathbf{w} ; \boldsymbol{\psi})}{\partial \boldsymbol{\psi}}\right\}^{\top}\right) f_{\mathbf{w}}(\mathbf{w} ; \boldsymbol{\psi}) d \mathbf{w}+ \\
& {\left[\int_{\mathscr{R}(\mathbf{y})} f_{\mathbf{w}}(\mathbf{w} ; \boldsymbol{\psi}) d \mathbf{w}\right]^{-1} \int_{\mathscr{R}(\mathbf{y})}\left\{\frac{1}{f_{\mathbf{w}}(\mathbf{w} ; \boldsymbol{\psi})} \frac{\partial f_{\mathbf{w}}(\mathbf{w} ; \boldsymbol{\psi})}{\partial \boldsymbol{\psi}}\right\} \times} \\
& \left\{\frac{1}{f_{\mathbf{w}}(\mathbf{w} ; \boldsymbol{\psi})} \frac{\partial f_{\mathbf{w}}(\mathbf{w} ; \boldsymbol{\psi})}{\partial \boldsymbol{\psi}}\right\}^{\top} f_{\mathbf{w}}(\mathbf{w} ; \boldsymbol{\psi}) d \mathbf{w}= \\
& E\left[\frac{\partial^{2} \log L(\boldsymbol{\psi} ; \mathbf{w})}{\partial \boldsymbol{\psi} \partial \boldsymbol{\psi} \boldsymbol{\psi}^{\top}}-\left\{\frac{\partial \log L_{c}(\boldsymbol{\psi} ; \mathbf{w})}{\partial \boldsymbol{\psi}}\right\}\left\{\frac{\partial \log L_{c}(\boldsymbol{\psi} ; \mathbf{w})}{\partial \boldsymbol{\psi}}\right\}^{\top} \mid \mathbf{Y}=\mathbf{y}\right] .
\end{aligned}
$$


Therefore

$$
\begin{aligned}
\frac{\partial^{2} \log L(\boldsymbol{\psi} ; \mathbf{y})}{\partial \boldsymbol{\psi} \partial \boldsymbol{\psi}^{\top}}= & E\left[\frac{\partial^{2} \log L_{c}(\boldsymbol{\psi} ; \mathbf{w})}{\partial \boldsymbol{\psi} \partial \boldsymbol{\psi}^{\top}}-\left\{\frac{\partial \log L_{c}(\boldsymbol{\psi} ; \mathbf{w})}{\partial \boldsymbol{\psi}}\right\}\left\{\frac{\partial \log L_{c}(\boldsymbol{\psi} ; \mathbf{w})}{\partial \boldsymbol{\psi}}\right\}^{\top} \mid \mathbf{Y}=\mathbf{y}\right]- \\
& E\left[\frac{\partial \log L_{c}(\boldsymbol{\psi} ; \mathbf{w})}{\partial \boldsymbol{\psi}} \mid \mathbf{Y}=\mathbf{y}\right] E\left[\frac{\partial \log L_{c}(\boldsymbol{\psi} ; \mathbf{w})}{\partial \boldsymbol{\psi}} \mid \mathbf{Y}=\mathbf{y}\right]^{\top}
\end{aligned}
$$

The ML equations are then as follows:

$$
E\left[\frac{\partial \log L_{c}(\boldsymbol{\psi} ; \mathbf{w})}{\partial \boldsymbol{\psi}} \mid \mathbf{Y}=\mathbf{y}\right]=\mathbf{0}
$$

Equations (3.7) and (3.9) may be obtained from Equation (3.18) by setting $\boldsymbol{\psi}=$ $\left(\boldsymbol{\beta}, \tau^{2}, \mathbf{D}\right), \mathbf{w}=(\mathbf{y}, \mathbf{u})$ and considering

$$
f_{\mathbf{w}}(\mathbf{w} ; \boldsymbol{\psi})=f_{\mathbf{U}}(\mathbf{u} ; \mathbf{D}) \prod_{i=1}^{K} \prod_{j=1}^{n_{i}} \exp \left(\frac{y_{i j} \gamma_{i}\left(\mathbf{u}_{i}, \boldsymbol{\beta}\right)-b\left(\gamma_{i}\left(\mathbf{u}_{i}, \boldsymbol{\beta}\right)\right)}{\tau^{2}}-c\left(y_{i j}, \tau\right)\right)
$$

ML equations of missing data problems (i.e. Equation (3.18)) are usually solved iteratively with the EM algorithm [19].

\subsubsection{Generalized Estimating Equations}

The GEE approach has been introduced by Liang and Zeger [2]. It is based on the assumption that within each cluster, individual observations are independent and follow a marginal Generalized Linear Model as given by Equations (2.1) and (2.2) Then, $\boldsymbol{\beta}$ is estimated based on the GLM ML equation given by Equation (2.3) Liang and Zeger have shown the consistency of this estimator under appropriate regularity conditions.

They have also described an iterative procedure to jointly estimate the variancecovariance parameters and the regression coefficients $\boldsymbol{\beta}$ with greater efficiency, using the concept of working correlation matrix. The working correlation matrix describes 
the structure of the correlation matrix. When all clusters have an identical size $n$, the working correlation matrix is a known function $R(\boldsymbol{\alpha})$ of unknown parameters $\boldsymbol{\alpha}$ to be estimated. In the independence case, $R(\boldsymbol{\alpha})$ is simply the identity matrix. Other examples are given in [2]. Under appropriate regularity conditions, the knowledge of $R\left(\right.$ ) suffices to obtain consistent estimates of $\boldsymbol{\beta}$, and of the variance components $\tau^{2}$ and $\boldsymbol{\alpha}$. The estimation procedure uses

- $\hat{\boldsymbol{\alpha}}$ a $K^{1 / 2}$-consistent estimator of $\boldsymbol{\alpha}$ when both $\boldsymbol{\beta}$ and $\tau^{2}$ are known

- $\hat{\tau}^{2}$ a $K^{1 / 2}$-consistent estimator of $\tau^{2}$ when both $\boldsymbol{\beta}$ and $\boldsymbol{\alpha}$ are known

- The General Estimating Equations given in [2]. These equations are an extension of Equation (2.3).

The GEE approach requires few distributional assumptions about random effects. As a result it is robust to misspecified random effects distributions. This strength is also a disadvantage for other uses of GLMMs, such as prediction in longitudinal studies.

\subsubsection{Penalized Quasi Likelihood (PQL)}

In this approach, the parameters are estimated iteratively based on an approximating Linear Mixed Model. The approximating LMM is obtained from Equation 3.7, based on a first-order Taylor-series expansion of $\mu_{i}, g_{\mu}\left(\mu_{i}\right)$ and $\mathbf{W}^{\star}(\mathbf{u})$ around $\mathbf{0}$, the mean of the random effects $\mathbf{u}_{i}$. The PQL approach does not always produce good estimates. Problems have been observed with binary data in small clusters. 


\subsection{The EM algorithm and its variants}

\subsubsection{Basic algorithm}

In this section we borrow the notation of Section 3.2.2. The goal is to compute the solutions of Equation (3.18). The general idea of the EM algorithm is as follows [19]. For convenience, define the function

$$
Q\left(\boldsymbol{\psi}^{\prime}, \boldsymbol{\psi} ; \mathbf{y}\right):=\frac{\int_{\mathscr{R}(\mathbf{y})} \log f_{\mathbf{w}}\left(\mathbf{w} ; \boldsymbol{\psi}^{\prime}\right) f_{\mathbf{w}}(\mathbf{w} ; \boldsymbol{\psi}) d \mathbf{w}}{\int_{\mathscr{R}(\mathbf{y})} f_{\mathbf{w}}(\mathbf{w} ; \boldsymbol{\psi}) d \mathbf{w}}
$$

With a slight abuse of notation we may also write $Q\left(\boldsymbol{\psi}^{\prime}, \boldsymbol{\psi} ; \mathbf{y}\right)=$ $E\left[\log f_{\mathbf{w}}\left(\mathbf{w} ; \boldsymbol{\psi}^{\prime}\right) \mid \mathbf{Y}=\mathbf{y}, \boldsymbol{\psi}\right]$ At iteration $r \geq 1$ the EM algorithm selects $\boldsymbol{\psi}^{(r)}$ to maximize $Q\left(\boldsymbol{\psi}, \boldsymbol{\psi}^{(r-1)} ; \mathbf{y}\right)$.

Suppose $f_{\mathrm{w}}$ belongs to the curved exponential family, i.e.

$$
f_{\mathbf{w}}(\mathbf{w} ; \boldsymbol{\psi})=\frac{1}{a(\boldsymbol{\psi})} \exp \left(\mathbf{c}(\boldsymbol{\psi})^{\top} \mathbf{T}(\mathbf{w})\right)
$$

for some scalar function $a()$ and some vector functions $\mathbf{c}()$ and $\mathbf{T}()$. Then iteration $r$ of the EM algorithm comprises of two steps:

1. E step: compute the following conditional expectation:

$$
E\left[\mathbf{T}(\mathbf{w}) \mid \mathbf{Y}=\mathbf{y}, \boldsymbol{\psi}^{(r)}\right]:=\frac{\int_{\mathscr{R}(\mathbf{y})} \mathbf{T}(\mathbf{w}) f_{\mathbf{w}}\left(\mathbf{w} ; \boldsymbol{\psi}^{(r)}\right) d \mathbf{w}}{\int_{\mathscr{R}(\mathbf{y})} f_{\mathbf{w}}\left(\mathbf{w} ; \boldsymbol{\psi}^{(r)}\right) d \mathbf{w}} .
$$

2. $M$ step: select $\boldsymbol{\psi}^{(r+1)}$ to maximize

$$
Q\left(\boldsymbol{\psi}, \boldsymbol{\psi}^{(r)} ; \mathbf{y}\right)=-\log a(\boldsymbol{\psi})+\mathbf{c}(\boldsymbol{\psi})^{\top} E\left[\mathbf{T}(\mathbf{w}) \mid \mathbf{Y}=\mathbf{y}, \boldsymbol{\psi}^{(r)}\right]
$$


The EM algorithm takes an even simpler form when $f_{\mathrm{w}}$ has a regular form, i.e.

$$
f_{\mathbf{w}}(\mathbf{w} ; \boldsymbol{\psi})=\frac{1}{a(\boldsymbol{\psi})} d(\mathbf{w}) \exp \left(\boldsymbol{\psi} \mathbf{T}(\mathbf{w})^{\top}\right)
$$

In this case $\mathbf{T}(\mathbf{w})$ is a vector of sufficient statistics for $\boldsymbol{\psi}$, and iteration $r$ has the following form [19]:

1. E step: estimate $\mathbf{T}(\mathbf{w})$ by

$$
\mathbf{T}^{(r)}=E\left[\mathbf{T}(\mathbf{w}) \mid \mathbf{Y}=\mathbf{y}, \boldsymbol{\psi}^{(r)}\right]
$$

2. $M$ step: determine $\boldsymbol{\psi}^{(r+1)}$ to satisfy

$$
\int \mathbf{T}(\mathbf{w}) f_{\mathbf{w}}(\mathbf{w} ; \boldsymbol{\psi}) d \mathbf{w}=\mathbf{T}^{(r)}
$$

Dempster et al. [19] have given sufficient conditions for the convergence of the sequence $\boldsymbol{\psi}^{(0)}, \boldsymbol{\psi}^{(1)}, \ldots$ to a limit, for a general class of EM algorithms which they have called Generalized EM (GEM) algorithms. A GEM algorithm is defined as follows:

Definition 1 Consider an algorithm that computes a sequence $\boldsymbol{\psi}^{(0)}, \boldsymbol{\psi}^{(1)}, \ldots$ of estimates of the parameters $\boldsymbol{\psi}$ based on the observed data $\mathbf{y}$, and a mapping $\mathbf{M}(.,$.$) such$ that

$$
\boldsymbol{\psi}^{(r+1)}=\mathbf{M}\left(\boldsymbol{\psi}^{(r)}, \mathbf{y}\right)
$$

Then the algorithm is a GEM if

$$
Q\left(\mathbf{M}\left(\boldsymbol{\psi}^{(r)}, \mathbf{y}\right), \boldsymbol{\psi}^{(r)} ; \mathbf{y}\right) \geq Q(\boldsymbol{\psi}, \boldsymbol{\psi} ; \mathbf{y})
$$

for all $\mathbf{y}$ and $\boldsymbol{\psi}$ in $\Omega$ the parameter space. 
Conditions for the convergence of GEM algorithms are given in the following theorem [19]:

Theorem 1 Suppose that $\boldsymbol{\psi}^{(r)}$ for $r=0,1,2, \ldots$ is an instance of a GEM algorithm such that

1. the sequence $L\left(\boldsymbol{\psi}^{(r)} ; \mathbf{y}\right)$ is bounded and

2.

$$
Q\left(\boldsymbol{\psi}^{(r+1)}, \boldsymbol{\psi}^{(r)} ; \mathbf{y}\right)-Q\left(\boldsymbol{\psi}^{(r)}, \boldsymbol{\psi}^{(r)} ; \mathbf{y}\right) \geq \lambda\left(\boldsymbol{\psi}^{(r+1)}-\boldsymbol{\psi}^{(r)}\right)\left(\boldsymbol{\psi}^{(r+1)}-\boldsymbol{\psi}^{(r)}\right)^{\top}
$$

for all $r$, for some scalar $\lambda>0$

Then the sequence $\boldsymbol{\psi}^{(r)}$ converges to some $\boldsymbol{\psi}^{*}$ in the closure of $\Omega$ the parameter space.

When $f_{\mathbf{w}}$ has a regular exponential form, Dempster et al. [19] have provided the following heuristic justification that each limit point of the EM algorithm coincides with a stationary point of the observed likelihood. Suppose the EM algorithm converges to a limit $\boldsymbol{\psi}^{*}$, then according to Equations (3.25) and (3.26), it satisfies the following equation:

$$
\frac{\int_{\mathscr{R}(\mathbf{y})} \mathbf{T}(\mathbf{w}) f_{\mathbf{w}}\left(\mathbf{w} ; \boldsymbol{\psi}^{*}\right) d \mathbf{w}}{\int_{\mathscr{R}(\mathbf{y})} f_{\mathbf{w}}\left(\mathbf{w} ; \boldsymbol{\psi}^{*}\right) d \mathbf{w}}=\int \mathbf{T}(\mathbf{w}) f_{\mathbf{w}}\left(\mathbf{w} ; \boldsymbol{\psi}^{*}\right) d \mathbf{w}
$$

However, we also have

$$
\frac{\partial \log L(\boldsymbol{\psi} ; \mathbf{y})}{\partial \boldsymbol{\psi}}=-\int \mathbf{T}(\mathbf{w}) f_{\mathbf{w}}\left(\mathbf{w} ; \boldsymbol{\psi}^{*}\right) d \mathbf{w}+\frac{\int_{\mathscr{R}(\mathbf{y})} \mathbf{T}(\mathbf{w}) f_{\mathbf{w}}\left(\mathbf{w} ; \boldsymbol{\psi}^{*}\right) d \mathbf{w}}{\int_{\mathscr{R}(\mathbf{y})} f_{\mathbf{w}}\left(\mathbf{w} ; \boldsymbol{\psi}^{*}\right) d \mathbf{w}}
$$


Indeed

$$
\begin{aligned}
\log L(\boldsymbol{\psi} ; \mathbf{w}) & =\log \left(\int_{\mathscr{R}(\mathbf{y})} d(\mathbf{w}) \exp \left(\boldsymbol{\psi} \mathbf{T}(\mathbf{w})^{\top}\right) d \mathbf{w}\right)-\log (a(\boldsymbol{\psi})) \\
& =\log \left(\int_{\mathscr{R}(\mathbf{y})} d(\mathbf{w}) \exp \left(\boldsymbol{\psi} \mathbf{T}(\mathbf{w})^{\top}\right) d \mathbf{w}\right)-\log \left(\int d(\mathbf{w}) \exp \left(\boldsymbol{\psi} \mathbf{T}(\mathbf{w})^{\top}\right) d \mathbf{w}\right)
\end{aligned}
$$

Therefore

$$
\begin{aligned}
\frac{\partial \log L(\boldsymbol{\psi} ; \mathbf{w})}{\partial \boldsymbol{\psi}} & =\frac{\int_{\mathscr{R}(\mathbf{y})} d(\mathbf{w}) \exp \left(\boldsymbol{\psi} \mathbf{T}(\mathbf{w})^{\top}\right) \mathbf{T}(\mathbf{w}) d \mathbf{w}}{\int_{\mathscr{R}(\mathbf{y})} d(\mathbf{w}) \exp \left(\boldsymbol{\psi} \mathbf{T}(\mathbf{w})^{\top}\right) d \mathbf{w}}-\frac{\int d(\mathbf{w}) \exp \left(\boldsymbol{\psi} \mathbf{T}(\mathbf{w})^{\top}\right) \mathbf{T}(\mathbf{w}) d \mathbf{w}}{\int d(\mathbf{w}) \exp \left(\boldsymbol{\psi} \mathbf{T}(\mathbf{w})^{\top}\right) d \mathbf{w}} \\
& =\frac{\int_{\mathscr{R}(\mathbf{y})} d(\mathbf{w}) \exp \left(\boldsymbol{\psi} \mathbf{T}(\mathbf{w})^{\top}\right) \mathbf{T}(\mathbf{w}) d \mathbf{w}}{\int_{\mathscr{R}(\mathbf{y})} d(\mathbf{w}) \exp \left(\boldsymbol{\psi} \mathbf{T}(\mathbf{w})^{\top}\right) d \mathbf{w}}-\int d(\mathbf{w}) \exp \left(\boldsymbol{\psi} \mathbf{T}(\mathbf{w})^{\top}\right) \mathbf{T}(\mathbf{w}) d \mathbf{w}
\end{aligned}
$$

Q.E.D.

The EM algorithm requires the evaluation of conditional expectations in each E step. In large and complex GLMMs,it means computing high-dimensional integrals. Montecarlo methods are commonly used to evaluate such integrals. Even then, the resulting algorithm may be too slow for any practical application. The algorithm may be further accelerated by stochastic approximation steps leading to SAEM algorithms. The M step may be another source of difficulty, especially when it is itself iterative. In some cases, a faster algorithm may be obtained by replacing the maximization with a sequence of conditional maximizations steps as in the ECM algorithm. These different improvements are discussed in the following sections. 


\subsubsection{ECM algorithm}

The ECM algorithm was proposed by X. Meng and D. Rubin [24]. It is a variant of the EM algorithm, which replaces the M-step by many simpler constrained maximization steps. In each constrained maximization step, the likelihood is increased. The ECM algorithm belongs to the family of GEM algorithms described in the previous section. In a simple example of ECM algorithm, each constrained-maximization step maximizes the function $Q(.,$.$) with respect to one coordinate at a time the other coordi-$ nates being fixed to their values in the previous step. Suppose $\boldsymbol{\psi}$ is a $S \times 1$ vector of parameters. In iteration $r+1$, the $\mathrm{M}$ step is divided into $S$ constrained-maximization steps. Let $\boldsymbol{\psi}^{(r+1, s)}$ denote the estimate in the $s$-th constrained maximization steps. Also denote by $\psi_{s}$ the $s$-th coordinate of $\boldsymbol{\psi}$ and by $\boldsymbol{\psi}_{(s)}$ the vector comprising of the other coordinates (excluding $\psi_{s}$ ). The $\mathrm{M}$ step is as follows.

1. Select $\boldsymbol{\psi}^{(r+1,1)}$ to maximize $Q\left(\boldsymbol{\psi}, \boldsymbol{\psi}^{(r)} ; \mathbf{y}\right)$ subject to $\boldsymbol{\psi}_{(1)}=\boldsymbol{\psi}_{(1)}^{(r)}$

2. For $s=2, \ldots, S$, select $\boldsymbol{\psi}^{(r+1, s)}$ to maximize $Q\left(\boldsymbol{\psi}, \boldsymbol{\psi}^{(r)} ; \mathbf{y}\right)$ subject to $\boldsymbol{\psi}_{(s)}=$ $\psi_{(s)}^{(r+1, s-1)}$

3. Set $\boldsymbol{\psi}^{(r+1)}=\boldsymbol{\psi}^{(r+1, S)}$

In the above example, each constrained maximization is a simpler one-dimensional maximization problem.

A more general formulation of the algorithm considers arbitrary constraints given by a family of vector functions $\mathbf{g}_{1}(), \ldots, \mathbf{g}_{S}()$, where $S$ need not coincide with the dimension of the parameter vector $\boldsymbol{\psi}$. In this case the $\mathrm{M}$ step has the following form:

1. Select $\boldsymbol{\psi}^{(r+1,1)}$ to maximize $Q\left(\boldsymbol{\psi}, \boldsymbol{\psi}^{(r)} ; \mathbf{y}\right)$ subject to $\mathrm{g}_{1}(\boldsymbol{\psi})=\mathrm{g}_{1}\left(\boldsymbol{\psi}^{(r)}\right)$

2. For $s=2, \ldots, S$, select $\boldsymbol{\psi}^{(r+1, s)}$ to maximize $Q\left(\boldsymbol{\psi}, \boldsymbol{\psi}^{(r)} ; \mathbf{y}\right)$ subject to $\mathrm{g}_{s}(\boldsymbol{\psi})=$ $\mathrm{g}_{s}\left(\boldsymbol{\psi}^{(r+1, s-1)}\right)$ 
3. Set $\boldsymbol{\psi}^{(r+1)}=\boldsymbol{\psi}^{(r+1, S)}$

The preceding example is the special case $\mathbf{g}_{s}(\boldsymbol{\psi})=\boldsymbol{\psi}_{(s)}$. By construction, the ECM algorithm is a GEM.

The stationary points of the ECM algorithm are also stationary points of the observed log-likelihood when the transformations $\mathbf{g}_{1}, \ldots, \mathbf{g}_{S}$ are space-filling [24]. Meng and Rubin have provided a formal but technical definition of space-filling transformations (see [24] for details). Informally, space-filling transformations do not constrain the search in all possible directions of the original parameters space $\Omega$. When the transformations are continuously differentiable, the transformations are space-filling if the following simple sufficient condition is met.

Sufficient condition for space filling transformations: Suppose that for all $s=1, \ldots, S$ the gradient $\nabla \mathbf{g}_{s}(\boldsymbol{\psi})=\frac{\partial \mathbf{g}_{s}(\boldsymbol{\psi})}{\partial \boldsymbol{\psi}}$ is of full rank, wen $\boldsymbol{\psi}$ is in the interior of the parameter space $\Omega$, and let $J_{s}(\boldsymbol{\psi})$ denote the linear space spanned by the column vector of the gradient. Then the transformations are space filling when the following condition holds for all $\boldsymbol{\psi}$ in the interior of the parameter space.

$$
\bigcap_{s=1}^{S} J_{s}(\boldsymbol{\psi})=\{\mathbf{0}\}
$$

The following theorem was proved by Meng and Rubin [24].

Theorem 2 Suppose a space-filling family of functions $\mathbf{g}_{1}, \ldots, \mathbf{g}_{S}$, and a unique solution for each constrained maximization step. Then all limits points of any ECM sequence $\left\{\boldsymbol{\psi}^{(r)}, r=0,1, \ldots\right\}$ are sationary points of the observed log-likelihood.

The ECM sequence converges to the global maximum of the observed log-likelihood under additional conditions given in the following corollary (see [24]).

Corollary 1 Suppose that the observed log-likelihood is unimodal in $\boldsymbol{\psi}$ in the parameter space $\Omega$, with a single stationary point $\boldsymbol{\psi}^{*}$. Then any ECM sequence 
$\left\{\boldsymbol{\psi}^{(r)}, r=0,1, \ldots\right\}$ converges to $\boldsymbol{\psi}^{*}$ if the family $\mathbf{g}_{1}, \ldots, \mathbf{g}_{S}$ is space filling and either of the following two conditions are met:

1. Each constrained maximization step has a unique solution

2. $\frac{\partial Q\left(\boldsymbol{\psi}^{\prime}, \boldsymbol{\psi} ; \mathbf{y}\right)}{\partial \boldsymbol{\psi}^{\prime}}$ is continuous in both $\boldsymbol{\psi}$ and $\boldsymbol{\psi}^{\prime}$, and $\nabla \mathbf{g}_{s}$ is continuous for all $s=$ $1, \ldots, S$.

The need for the space filling condition may be heuristically justified as follows. Let $\boldsymbol{\lambda}^{(r, s)}$ the vector of lagrange multipliers associated with the $s$-th constrained maximization in iteration $r$. Under appropriate regularity conditions, we have

$$
\begin{aligned}
& \left.\frac{\partial Q\left(\boldsymbol{\psi}, \boldsymbol{\psi}^{(r)}, \mathrm{y}\right)}{\partial \boldsymbol{\psi}}\right|_{\boldsymbol{\psi}=\boldsymbol{\psi}^{(r+1,1)}}+\nabla \mathrm{g}_{1}\left(\boldsymbol{\psi}^{(r+1,1)}\right) \boldsymbol{\lambda}^{(r+1,1)}=0 . \\
& \left.\frac{\partial Q\left(\boldsymbol{\psi}, \boldsymbol{\psi}^{(r)}, \mathbf{y}\right)}{\partial \boldsymbol{\psi}}\right|_{\boldsymbol{\psi}=\boldsymbol{\psi}^{(r+1, s)}}+\nabla \mathrm{g}_{s}\left(\boldsymbol{\psi}^{(r+1, s)}\right) \boldsymbol{\lambda}^{(r+1, s)}=0,2 \leq s \leq S .
\end{aligned}
$$

Suppose $\boldsymbol{\psi}^{*}$ is a limit point of the ECM sequence. Then there exist lagrange-multiplier vectors $\boldsymbol{\lambda}_{1}, \ldots, \boldsymbol{\lambda}_{S}$ such that

$$
\left.\frac{\partial Q\left(\boldsymbol{\psi}, \boldsymbol{\psi}^{*}, \mathbf{y}\right)}{\partial \boldsymbol{\psi}}\right|_{\boldsymbol{\psi}=\boldsymbol{\psi}^{*}}+\nabla \mathbf{g}_{s}\left(\boldsymbol{\psi}^{*}\right) \boldsymbol{\lambda}_{s}=\mathbf{0}, \quad 1 \leq s \leq S
$$

The above equation implies $\left.\frac{\partial Q\left(\boldsymbol{\psi}, \boldsymbol{\psi}^{*}, \mathbf{y}\right)}{\partial \boldsymbol{\psi}}\right|_{\boldsymbol{\psi}=\boldsymbol{\psi}^{*}} \in \bigcap_{s=1}^{S} J_{s}\left(\boldsymbol{\psi}^{*}\right)$. When $\bigcap_{s=1}^{S} J_{s}\left(\boldsymbol{\psi}^{*}\right)=$ $\{0\}$, we have

$$
\left.\frac{\partial \log L(\boldsymbol{\psi} ; \mathrm{y})}{\partial \boldsymbol{\psi}}\right|_{\boldsymbol{\psi}=\boldsymbol{\psi}^{*}}=\left.\frac{\partial Q\left(\boldsymbol{\psi}, \boldsymbol{\psi}^{*}, \mathrm{y}\right)}{\partial \boldsymbol{\psi}}\right|_{\boldsymbol{\psi}=\boldsymbol{\psi}^{*}}=\mathbf{0}
$$

Hence $\boldsymbol{\psi}^{*}$ is a stationary point of the observed log-likelihood.

\subsubsection{SAEM and SAECM algorithms}

For complex models, intensive computations are needed to evaluate conditional expectations in the E-step. Stochastic approximation (SA) addresses this problem. It 
may be applied to the EM algorithm resulting in an SAEM procedure or to the ECM algorithm resulting in a SAECM procedure. Delyon et al. have described these algorithms and analysed their properties in details [22]. A summary of this work is provided in the following paragraphs.

SAEM algorithm: for a more general description, let $\mathbf{y}$ denote the observed data, $\mathbf{w}$ the complete data. In the previous paragrah, we had $\mathbf{w}=(\mathbf{y}, \mathbf{u})$. It is assumed that the complete data is distributed according to the density ${ }^{1} f_{\mathrm{w}}(\mathbf{w} ; \boldsymbol{\psi})$, depending on the vector of parameters $\boldsymbol{\psi}$ in $\Omega$. The density $f_{\mathrm{w}}$ is assumed to belong to the curved exponential family, i.e. it is of the form $f_{\mathbf{w}}(\mathbf{w} ; \boldsymbol{\psi})=\exp (-a(\boldsymbol{\psi})+\langle\mathbf{c}(\boldsymbol{\psi}), \mathbf{T}(\mathbf{w})\rangle)$, where $\langle.,$.$\rangle denotes the scalar product { }^{2}$. Also let $\mathscr{R}(\mathbf{y})$ denote the set of complete data for which the observed data is $\mathbf{y}$ with non-zero probability. Define

$$
Q(\boldsymbol{\psi}, \mathbf{t})=-a(\boldsymbol{\psi})+\langle\mathbf{c}(\boldsymbol{\psi}), \mathbf{t}\rangle
$$

For a given $\mathbf{t} \in \mathscr{T}$ some open subset of $\mathbb{\|} \mathbf{I}^{m}$, define $\widehat{\boldsymbol{\psi}}(\mathbf{t})$ such that

$$
Q(\widehat{\boldsymbol{\psi}}(\mathbf{t}), \mathbf{t}) \geq Q\left(\boldsymbol{\psi}^{\prime}, \mathbf{t}\right), \quad \forall \boldsymbol{\psi}^{\prime} \in \Omega
$$

Under regularity conditions

$$
\frac{\partial Q(\widehat{\boldsymbol{\psi}}(\mathbf{t}), \mathbf{t})}{\partial \boldsymbol{\psi}}=\mathbf{0}, \quad \forall \mathbf{t} \in \mathscr{T}
$$

Define $\widehat{\mathbf{t}}(\boldsymbol{\psi} ; \mathbf{y})=E[\mathrm{~T}(\mathbf{w}) \mid \mathbf{Y}=\mathbf{y}, \boldsymbol{\psi}]$. Let $\boldsymbol{\psi}^{*}$ denote the MLE of $\boldsymbol{\psi}$ and define $\mathbf{t}^{*}:=\widehat{\mathbf{t}}\left(\boldsymbol{\psi}^{*} ; \mathbf{y}\right)$. Under regularity conditions

$$
\frac{\partial Q\left(\boldsymbol{\psi}^{*}, \mathbf{t}^{*}\right)}{\partial \boldsymbol{\psi}}=\mathbf{0}, \text { and } \mathbf{t}^{*}-E\left[\mathbf{T}(\mathbf{w}) \mid \mathbf{Y}=\mathbf{y}, \boldsymbol{\psi}^{*}\right]=\mathbf{0}
$$

\footnotetext{
${ }^{1}$ Delyon et al. consider a more general formulation were the density $f_{\mathrm{w}}$ is defined with respect to a $\sigma$-finite Borel measure $\mu$ on $\underline{\| l^{l}}$

${ }^{2}$ For example, in $\mathbb{\| \mathbf { R } ^ { m \times n }}$ define $\langle\mathbf{A}, \mathbf{B}\rangle:=\operatorname{tr}\left(\mathbf{A}^{\top} \mathbf{B}\right)=\operatorname{tr}\left(\mathbf{B} \mathbf{A}^{\top}\right)$
} 
The first equation corresponds to the M-step, while the second equation corresponds to the E step. Knowledge of $\mathbf{t}^{*}$ is also sufficient to characterize the MLE through the mapping $\boldsymbol{\psi}^{*}=\widehat{\boldsymbol{\psi}}\left(\mathbf{t}^{*}\right)$. Therefore $\mathbf{t}^{*}$ is characterized by the following equation.

$$
\mathbf{t}^{*}=E\left[\mathbf{T}(\mathbf{w}) \mid \mathbf{Y}=\mathbf{y}, \widehat{\psi}\left(\mathbf{t}^{*}\right)\right]
$$

The idea of $\mathrm{SA}$ is to solve the above equation iteratively by computing a sequence $\mathbf{T}^{0}, \mathbf{T}^{(1)}, \ldots$, converging to the estimate $\mathbf{t}^{*}$. This sequence is computed according to the following equation where $\left\{\gamma_{k}\right\}_{k \geq 0}$ is a positive sequence such that $\sum_{k=1}^{\infty} \gamma_{k}=\infty$.

$$
\mathbf{T}^{(k+1)}=\mathbf{T}^{(k)}+\gamma_{k}\left[\mathbf{T}\left(\mathbf{W}_{k+1}\right)-\mathbf{T}^{(k)}\right]
$$

where $\mathbf{Z}_{k+1}$ is a random variable drawn independently of the past, according to the conditional distribution of the complete data given the observed data and as if $\boldsymbol{\psi}^{(k)}$ were the value of the distribution parameter $\boldsymbol{\psi}$. Then $\boldsymbol{\psi}^{(k)}=\widehat{\boldsymbol{\psi}}\left(\mathbf{T}^{(k)}\right)$ also defines a sequence of estimates of $\boldsymbol{\psi}^{*}$. We also have

$$
\mathbf{T}^{(k+1)}=\mathbf{T}^{(k)}+\gamma_{k} \mathbf{h}\left(\mathbf{T}^{(k)}\right)+\gamma_{k} \mathbf{E}_{k}
$$

where $\mathbf{h}\left(\mathbf{T}^{(k)}\right)=E\left[\mathbf{T}(\mathbf{w}) \mid \mathbf{Y}=\mathbf{y}, \widehat{\boldsymbol{\psi}}\left(\mathbf{T}^{(k)}\right)\right]-\mathbf{T}^{(k)}=\gamma_{k}^{-1}\left(E\left[\mathbf{T}^{(k+1)} \mid \mathbf{T}^{(k)}\right]-\mathbf{T}^{(k)}\right)$ is the mean field and $\mathbf{E}_{k}=\mathbf{T}\left(\mathbf{W}_{k+1}\right)-E\left[\mathbf{T}(\mathbf{w}) \mid \mathbf{Y}=\mathbf{y}, \widehat{\boldsymbol{\psi}}\left(\mathbf{T}^{(k)}\right)\right]$ is a zero mean random variable (also called random excitation [22]).

Let $\log L(\boldsymbol{\psi} ; \mathbf{y})$ denote the observed data log-likelihood and define $V(\mathbf{t})=$ $-\log L(\widehat{\boldsymbol{\psi}}(\mathbf{t}) ; \mathbf{y})$. Under regularity conditions (e.g. the mapping $\mathbf{t} \mapsto \widehat{\boldsymbol{\psi}}(\mathbf{t})$ must be continuously differentiable a sufficient number of times), the mean field $\mathbf{h}()$ satisfies the following important property [22]:

$$
\left\langle\frac{\partial V(\mathbf{t})}{\partial \mathbf{t}}, \mathbf{h}(\mathbf{t})\right\rangle \leq 0, \forall \mathbf{t} \in \mathscr{T} .
$$


Therefore when $k$ is sufficiently large, we have

$$
\begin{aligned}
-E\left[\log L\left(\boldsymbol{\psi}^{(k+1)} ; \mathbf{y}\right) \mid \mathbf{T}^{(k)}\right]+\log L\left(\boldsymbol{\psi}^{(k)} ; \mathbf{y}\right) & =E\left[V\left(\mathbf{T}^{(k+1)}\right) \mid \mathbf{T}^{(k)}\right]-V\left(\mathbf{T}^{(k)}\right) \\
& =\left|\frac{\partial V\left(\mathbf{T}^{(k)}\right)}{\partial \mathbf{t}}, \mathbf{h}\left(\mathbf{T}^{(k)}\right)\right\rangle+r_{k} \\
& \leq 0 .
\end{aligned}
$$

where $r_{k}$ is a small remainder. This means that at each iteration the log-likelihood is increased on average.

Delyon et al. have studied the convergence of the SAEM algorithm in the following setting [22]:

C.1 The parameter space $\Omega$ is an open subset of $\mathbb{H}^{p}$

C.2 The function $\mathbf{T}()$ is a Borel function on $\mathbb{\| 2} l$ taking its values in an open subset $\mathscr{T}$ of $\| \mathbb{R}^{m}$

C.3 The convex hull of $\mathbf{T}\left(\| \mathbb{R}^{l}\right)$ is included in $\mathscr{T}$

C. $4 \int_{\|\left|\mathbb{R}^{\mid}\right|}|\mathrm{T}(\mathbf{w})| f_{\mathrm{w}}(\mathbf{w} ; \boldsymbol{\psi}) d \mathbf{w}<\infty$

C.5 The functions $a()$ and $\mathbf{c}()$ are twice-continuously differentiable on $\Omega$

C.6 The observed log-likelihood is continuously differentiable on $\Omega$ and

$$
\frac{\partial}{\partial \boldsymbol{\psi}} \int f_{\mathbf{w}}(\mathbf{w} ; \boldsymbol{\psi}) d \mathbf{w}=\int \frac{\partial f_{\mathbf{w}}(\mathbf{w} ; \boldsymbol{\psi})}{\partial \boldsymbol{\psi}} d \mathbf{w}
$$

C.7 The function $\mathbf{t} \mapsto \widehat{\psi}(\mathbf{t})$ exists and is continuously differentiable on $\mathscr{T}$

In this context, Delyon et al. have proved the following theorem regarding the convergence of the SAEM algorithm. 
Theorem 3 With probability 1, the sequence $\left\{\boldsymbol{\psi}^{(k)}\right\}_{k \geq 1}$ and $\left\{\mathbf{T}^{(k)}\right\}_{k \geq 1}$ respectively converge to $\boldsymbol{\psi}^{*}$ and $\mathbf{t}^{*}$ that are fixed points of the EM mapping equations, i.e.

$$
\boldsymbol{\psi}^{*}=\widehat{\boldsymbol{\psi}}\left(\widehat{\mathbf{t}}\left(\boldsymbol{\psi}^{*} ; \mathbf{y}\right)\right) \text {, and } \mathbf{t}^{*}=\widehat{\mathbf{t}}\left(\widehat{\boldsymbol{\psi}}\left(\mathbf{t}^{*}\right) ; \mathbf{y}\right)
$$

provided the following conditions are met:

C.8 For all $k>0, \gamma_{k} \in[0,1], \sum_{k=1}^{\infty} \gamma_{k}=\infty$ and $\sum_{k=1}^{\infty} \gamma_{k}^{2}<\infty$

C.9 The observed log-likelihood is $m$ ( $m$ is the dimension of $\mathbf{T}(\mathbf{w})$ ) times continuously differentiable

C.10 The mapping $\mathbf{t} \rightarrow \boldsymbol{\psi}(\mathbf{t})$ is $m$ times continuously differentiable

C.11 Assuming that the random variables $\mathbf{T}^{(0)}, \mathbf{W}_{1}, \mathbf{W}_{2}, \ldots$ are defined on the same probability space, let $\mathscr{F}_{n}$ denote the $\sigma$-filed generated by $\mathbf{T}^{(0)}, \mathbf{W}_{1}, \mathbf{W}_{2}, \ldots, \mathbf{W}_{n}$. For all positive Borel function $\phi$

$$
E\left[\phi\left(\mathbf{W}_{k+1}\right) \mid \mathscr{F}_{k}\right]=\frac{\int_{\mathscr{R}(\mathbf{y})} \phi(\mathbf{w}) f_{\mathbf{w}}\left(\mathbf{w} ; \boldsymbol{\psi}^{(k)}\right) d \mathbf{w}}{\int_{\mathscr{R}(\mathbf{y})} f_{\mathbf{w}}\left(\mathbf{w} ; \boldsymbol{\psi}^{(k)}\right) d \mathbf{w}}
$$

C.12 For all $\boldsymbol{\psi} \in \Omega, \int_{\mathscr{R}(\mathbf{y})}\|\mathrm{T}(\mathbf{w})\|^{2} f_{\mathbf{w}}(\mathbf{w} ; \boldsymbol{\psi}) d \mathbf{w}<\infty$

C.13 The covariance function $\Gamma(\boldsymbol{\psi}):=\operatorname{Cov}_{\boldsymbol{\psi}}(\mathbf{T}(\mathbf{w}))$ is continuous w.r.t $\boldsymbol{\psi}$.

C.14 The closure of $\left\{\mathbf{T}^{(k)}\right\}_{k \geq 1}$ is a compact subset of $\mathscr{T}$ w.p.1

C.15 The stationary points of the observed log-likelihood are isolated, i.e. any compact susbset of $\Omega$ contains only a finite number of such points

In the above theorem, an important necessary condition for convergence is that the sequence $\left\{\mathbf{T}^{(k)}\right\}_{k \geq 1}$ must have a compact closure (condition C.14 in the theorem). It is not always satisfied and it may be sometimes difficult to check. In this case a truncated 
SAEM algorithm may be used, where the sequence is appropriately truncated (with an explicit stabilization device) as follows. Let $\left\{\mathscr{K}_{n}\right\}_{n \geq 0}$ be a sequence of compact subsets of $\mathscr{T}$ such that

$$
\mathscr{K}_{n} \subset \operatorname{int}\left(\mathscr{K}_{n+1}\right) \text {, and } \bigcup_{n=0}^{\infty} \mathscr{K}_{n}=\mathscr{T}
$$

where $\operatorname{int}\left(\mathscr{K}_{n+1}\right)$ is the interior of the set $\mathscr{K}_{n+1}$. Let $\left\{\mathbf{T}_{0}^{(k)}\right\}_{k \geq 1}$ be an arbitrary sequence of random variables taking their values in $\mathscr{K}_{0}$ :

$$
\begin{aligned}
& \widetilde{\mathbf{T}}^{(k+1)}=\mathbf{T}^{(k)}+\gamma_{k}\left[\mathbf{T}\left(\mathbf{W}_{k+1}\right)-\mathbf{T}^{(k)}\right] . \\
& \text { If } \widetilde{\mathbf{T}}^{(k+1)} \in \mathscr{K}_{n_{k}}\left\{\begin{array}{c}
\mathbf{T}^{(k+1)}=\widetilde{\mathbf{T}}^{(k+1)} \\
\boldsymbol{\psi}_{k+1}=\widehat{\boldsymbol{\psi}}\left(\mathbf{T}_{k+1}\right) \\
n_{k+1}=n_{k}
\end{array}\right. \\
& \text { If } \widetilde{\mathbf{T}}^{(k+1)} \notin \mathscr{K}_{n_{k}}\left\{\begin{array}{c}
\mathbf{T}^{(k+1)}=\mathbf{T}_{0}^{(k+1)} \\
\boldsymbol{\psi}_{k+1}=\widehat{\boldsymbol{\psi}}\left(\mathbf{T}_{k+1}\right) \\
n_{k+1}=n_{k}+1
\end{array}\right.
\end{aligned}
$$

Theorem 4 The sequence $\left\{\boldsymbol{\psi}^{(k)}\right\}_{k \geq 1}$ converges to some proper maximizer $\boldsymbol{\psi}^{*}$ of the observed log-likelihood provided that conditions C.8-C.11 and C.14 are satistifed in addition to the following conditions:

C.16 For every stationary point $\boldsymbol{\psi}^{*} \in \mathscr{L}$ the following matrices are positive definite

$$
E_{\boldsymbol{\psi}^{*}}\left[\left\{\frac{\partial Q\left(\boldsymbol{\psi}^{*}, \mathbf{T}(\mathbf{w})\right)}{\partial \boldsymbol{\psi}}\right\}^{\top} \frac{\partial Q\left(\boldsymbol{\psi}^{*}, \mathbf{T}(\mathbf{w})\right)}{\partial \boldsymbol{\psi}}\right], \text { and } \frac{\partial^{2} Q\left(\boldsymbol{\psi}^{*}, E_{\boldsymbol{\psi}^{*}}[\mathbf{T}(\mathbf{W})]\right)}{\partial \boldsymbol{\psi} \partial \boldsymbol{\psi}^{\top}}
$$


C.17 For some $\alpha>0, E_{\boldsymbol{\psi}}\left[\|\mathbf{T}(\mathbf{w})\|^{2+\alpha}\right]<\infty$ for all $\boldsymbol{\psi} \in \Omega$

C.18 The mapping $\boldsymbol{\psi} \mapsto \Gamma(\psi)=\operatorname{Cov}_{\boldsymbol{\psi}}(\mathbf{T}(\mathbf{w}))$ is continuous

C.19 The minimum eigenvalue of $\boldsymbol{\Gamma}(\boldsymbol{\psi})$ is bounded away from zero in any compact subset of $\mathscr{K} \in \Omega$

Suppose in addition that for some $\alpha \in(1 / 2,1)$

$$
\lim _{k \rightarrow \infty} k^{\alpha} \gamma_{k}=\gamma^{*}, \text { and } \frac{\gamma_{k}}{\gamma_{k+1}}=1+O\left(k^{-1}\right)
$$

and define

$$
\overline{\boldsymbol{\psi}}_{k+1}=\overline{\boldsymbol{\psi}}_{k}+k^{-1}\left(\boldsymbol{\psi}^{(k)}-\overline{\boldsymbol{\psi}}_{k}\right)
$$

Then

$$
\sqrt{n}\left(\overline{\boldsymbol{\psi}}_{n}-\boldsymbol{\psi}^{*}\right) \text { ㄲ}\left(\lim _{n \rightarrow \infty}\left\|\boldsymbol{\psi}_{n}-\boldsymbol{\psi}^{*}\right\|=0\right) \stackrel{d}{\rightarrow} \text { ill }\left(\lim _{n \rightarrow \infty}\left\|\boldsymbol{\psi}_{n}-\boldsymbol{\psi}^{*}\right\|=0\right) N\left(\mathbf{0}, \boldsymbol{\Sigma}^{*}\right)
$$

where

$$
\boldsymbol{\Sigma}^{*}:=\left[\frac{\partial^{2} l\left(\boldsymbol{\psi}^{*} ; \mathbf{y}\right)}{\partial \boldsymbol{\psi} \partial \boldsymbol{\psi}^{\top}}\right]^{-1}\left[\frac{\partial^{2} l\left(\boldsymbol{\psi}^{*} ; \mathbf{y}\right)}{\partial \boldsymbol{\psi} \partial \boldsymbol{\psi}^{\top}}-\frac{\partial^{2} Q\left(\boldsymbol{\psi}^{*}, \mathbf{t}^{*}\right)}{\partial \boldsymbol{\psi} \partial \boldsymbol{\psi}^{\top}}\right]\left[\frac{\partial^{2} l\left(\boldsymbol{\psi}^{*} ; \mathbf{y}\right)}{\partial \boldsymbol{\psi} \partial \boldsymbol{\psi}^{\top}}\right]^{-1}
$$

and $l(\boldsymbol{\psi} ; \mathbf{y})$ is the observed log-likelihood.

SAECM algorithm: the SA procedure is different for an ECM algorithm. Consider the following ECM procedure for the same missing data problem (as discussed in the SAEM case). In the E-step, compute

$$
\mathbf{T}^{(k+1)}=E\left[\mathbf{T}(\mathbf{w}) \mid \mathbf{Y}=\mathbf{y}, \boldsymbol{\psi}^{(k)}\right]
$$

In the M-step compute $\boldsymbol{\psi}^{(r+1)}$ as follows. Consider $S$ transformations $\mathbf{g}_{1}, \ldots, \mathbf{g}_{S}$ 
1. Select $\boldsymbol{\psi}^{(k+1,1)}$ to maximize $Q\left(\boldsymbol{\psi}, \mathbf{T}^{(k+1)}\right)$ subject to $\mathbf{g}_{1}(\boldsymbol{\psi})=\mathbf{g}_{1}\left(\boldsymbol{\psi}^{(k)}\right)$

2. For $s=2, \ldots, S$, select $\boldsymbol{\psi}^{(k+1, s)}$ to maximize $Q\left(\boldsymbol{\psi}, \mathbf{T}^{(k+1)}\right)$ subject to $\mathbf{g}_{s}(\boldsymbol{\psi})=$ $\mathbf{g}_{s}\left(\boldsymbol{\psi}^{(k+1, s-1)}\right)$

3. Set $\boldsymbol{\psi}^{(k+1)}=\boldsymbol{\psi}^{(k+1, S)}$

With SA, only the E step changes as follows:

$$
\mathbf{T}^{(k+1)}=\mathbf{T}^{(k)}+\gamma_{k}\left[\mathbf{T}\left(\mathbf{W}_{k+1}\right)-\mathbf{T}^{(k)}\right]
$$

where $\mathbf{Z}_{k+1}$ is a random variable drawn independently of the past, according to the conditional distribution of the complete data given the observed data and as if $\boldsymbol{\psi}^{(k)}$ were the value of the distribution parameter $\boldsymbol{\psi}$.

The following theorem gives conditions for the convergence of the SAECM algorithm (see Delyon et al. [22])

Theorem 5 Let $\mathscr{L}$ is the set of stationary points of the observed log-likelihood. With probability 1,

$$
\lim _{k \rightarrow \infty} d\left(\mathbf{T}^{(k)},\left\{\mathbf{t} \in \mathscr{T}: \frac{\partial V(\mathbf{t})}{\partial \mathbf{t}}=\mathbf{0}\right\}\right)=0
$$

and

$$
\lim _{k \rightarrow \infty} d\left(\boldsymbol{\psi}^{(k)}, \mathscr{L}\right)=0
$$

provided conditions C.1-C.6, C.8-C.14 are satisfied in addition to the following conditions:

C.20 For all $\mathbf{t} \in \mathscr{T}$ the function $\boldsymbol{\psi} \mapsto Q(\boldsymbol{\psi} ; \mathbf{t})$ has unique global maximum $\widehat{\boldsymbol{\psi}}(\mathbf{t})$ in $\Omega$ C.21 For $s=1, \ldots, S$ define the function $\psi^{(s)}: \mathscr{T} \times \Omega \rightarrow \Omega$ such that $\boldsymbol{\psi}^{(s)}\left(\mathbf{T}^{(k)}, \boldsymbol{\psi}^{(k)}\right)=$ $\boldsymbol{\psi}^{(k+1, s)}$. There exists a function $C: \mathscr{T} \times \Omega \rightarrow[0, \infty)$ and a function $\rho: \mathscr{T} \rightarrow$ $[0,1)$ such that

$$
\left\|\boldsymbol{\psi}^{(s)}(\mathbf{t}, \boldsymbol{\psi})-\boldsymbol{\psi}(\mathbf{t})\right\| \leq C(\mathbf{t} ; \boldsymbol{\psi}) \rho(\mathbf{t})^{s}
$$


In the above theorem, the Lyapunov function $\mathrm{V}()$ is defined as in the SAEM case. Also for some point $x$ and a set $A$ of a normed space, $d(x, A):=\inf \{d(x, y): y \in A\}$ denotes the distance of point $x$ to $A$.

As in the SAEM case, the SAECM iteration may be modified according to Equation (3.56) and Equation (3.57) to guarantee that the sequence $\left\{\mathbf{T}^{(k)}\right\}_{k \geq 1}$ has a compact closure. 


\section{Chapter 4}

\section{Multivariate probit models}

In the literature, the term multivariate probit models usually refers to GLMMs, where within each cluster, individual measurements involve multiple correlated binary responses, and a probit link function relates the mean of each response to the random effect and explanatory variables for the cluster. In this work, the term (multivariate probit models) refers to a larger class of models, which include GLMMs with the following characteristics for each measurement:

- Correlated responses

- At least one binary response with the probit link

- Zero or more continuous responses with the identity link

Gueorguieva and Agresti have described a multivariate latent response model, where the actual responses are obtained by thresholding a subset of the responses of an underlying Linear Mixed Model (LMM) [20]. In this model it is convenient to assume a normal distribution of random effects, because the ML equations have a simple form based on Louis's missing information principle. This advantage extends to the case where the random effects are distributed according to a mixture of normal distributions. 
The aim of this chapter is to obtain expressions for the ML equations, score and observed information matrix when the random effects follow a normal distribution or a normal mixture. To simplify the presentation, the detailed derivations have been postponed to Appendix B, and the expressions are given for the restricted model where each repeated measurement involves a continuous response and a binary response. The results may be extended to an arbitrary number of responses in a straightforward manner. Another reason for working with the resctricted model is the comparison with previous work on the same model by R. Gueorguieva and A. Agresti [20].

\subsection{Random effects with a normal distribution}

Consider an experiment with $K$ clusters. For cluster $i$ define the following:

- $n_{i}$ denote the number of measurements

- $\mathbf{u}_{i}$ the $s \times 1$ random effect assumed to be independent $N(\mathbf{0}, \boldsymbol{\Sigma})$

- $\mathbf{x}_{i}$ the $(q / 2) \times 1$ vector of covariates for the fixed effects

- $\mathbf{z}_{i}$ the vector of coefficients for the random effect,

- $\mathbf{y}_{i j}^{*}$ and $\mathbf{y}_{i j}$ respectively the $2 \times 1$ latent response and observed response vectors for the $j$-th repeated measurement.

- $\boldsymbol{\epsilon}_{i j}$ the random error in the model, for the $j$-th repeated measurement, and assumed to be independent $N\left(\mathbf{0}, \boldsymbol{\Sigma}_{e}\right)$ 
Typically $\boldsymbol{\Sigma}$ and $\boldsymbol{\Sigma}_{e}$ are functions of variance components. For example, the covariance of the errors may have the following form:

$$
\boldsymbol{\Sigma}_{e}:=\boldsymbol{\Sigma}_{e}\left(\sigma_{e}, \rho_{e}\right)=\left[\begin{array}{cc}
\sigma_{e}^{2} & \rho_{e} \sigma_{e}^{2} \\
\rho_{e} \sigma_{e}^{2} & \sigma_{e}^{2}
\end{array}\right]
$$

where $\sigma_{e}^{2}$ is the variance of the random errors, and $\rho_{e}$ is the correlation between the random errors for the continuous and discrete response. Let $\boldsymbol{\beta}$ denote the $q \times 1$ vector of regression coefficients. For convenience define $\mathbf{1}_{n \times m}$ as the $n \times m$ matrix with all ones, $\mathbf{I}_{n}$ the $n \times n$ identity matrix and $\mathbf{x}_{i j}:=\mathbf{x}_{i}^{\top} \otimes \mathbf{I}_{2}$. Let us define

$$
\begin{aligned}
& \mathbf{y}_{i j}^{*}=\left[\begin{array}{c}
y_{i j 1}^{*} \\
y_{i j 2}^{*}
\end{array}\right]=\mathbf{x}_{i j} \boldsymbol{\beta}+\mathbf{z}_{i j} \mathbf{u}_{i}+\boldsymbol{\epsilon}_{i j} . \\
& \mathbf{y}_{i j}=\left[\begin{array}{l}
y_{i j 1} \\
y_{i j 2}
\end{array}\right]=\left[\begin{array}{c}
y_{i j 1}^{*} \\
I\left\{y_{i j 2}^{*}>0\right\}
\end{array}\right],
\end{aligned}
$$

where $\mathbf{y}_{i j}$ is the observed data. For additional convenience, define

$$
\mathbf{Y}_{i}^{*}=\left[\begin{array}{c}
Y_{i 1}^{*} \\
\vdots \\
Y_{i n_{i}}^{*}
\end{array}\right], \quad \mathbf{Y}_{i}=\left[\begin{array}{c}
Y_{i 1} \\
\vdots \\
Y_{i n_{i}}
\end{array}\right], \quad \mathbf{Y}^{*}=\left[\begin{array}{c}
\mathbf{Y}_{1}^{*} \\
\vdots \\
\mathbf{Y}_{n}^{*}
\end{array}\right], \quad \mathbf{Y}=\left[\begin{array}{c}
\mathbf{Y}_{1} \\
\vdots \\
\mathbf{Y}_{n}
\end{array}\right], \quad \mathbf{u}=\left[\begin{array}{c}
\mathbf{u}_{1} \\
\vdots \\
\mathbf{u}_{n}
\end{array}\right]
$$

For fixed $\mathbf{y}_{i j}^{*}$ and $\mathbf{u}_{i}$ define the following function of $\boldsymbol{\beta}$ :

$$
\boldsymbol{\epsilon}_{i j}(\boldsymbol{\beta}):=\mathbf{y}_{i j}^{*}-\mathbf{x}_{i j} \boldsymbol{\beta}-\mathbf{z}_{i j} \mathbf{u}_{i}
$$


Hence the symbol $\boldsymbol{\epsilon}_{i j}$ will either refer to the random vector associated with measurement errors or to the function defined above, depending on the context. Define the complete data as $\left(\mathbf{Y}^{\star}, \mathbf{u}\right)$. For the complete data and observed data ML equations have been previously obtained by R. Gueorguieva and A. Agresti in [20]. For the complete data, expressions for the score and observed information matrix have been obtained by McCulloch in some special cases [1]. However these results are not suffciently general for our model.

The derivation of score and observed information quickly becomes tedious. The task is greatly facilitated by proper conventions for matrix differentiation as suggested by T. Kollo in [25]. These conventions are summarized in Appendix A, to which the reader is referred. The details are provided in Appendix B

\subsubsection{Complete data}

Let $\boldsymbol{\psi}$ denote the vector of parameters that include $\boldsymbol{\beta}, \boldsymbol{\Sigma}$ and $\boldsymbol{\Sigma}_{e}$. The complete data log-likelihood has the following expression:

$$
\log L_{c}\left(\boldsymbol{\psi} ; \mathbf{u}, \mathbf{y}^{*}\right)=-\frac{1}{2} \sum_{i=1}^{K} \sum_{j=1}^{n_{i}} \log \left|\boldsymbol{\Sigma}_{e}\right|-\frac{1}{2} \sum_{i=1}^{K} \sum_{j=1}^{n_{i}} \boldsymbol{\epsilon}_{i j}^{\top} \boldsymbol{\Sigma}_{e}^{-1} \boldsymbol{\epsilon}_{i j}-\frac{1}{2} \sum_{i=1}^{K} \log |\boldsymbol{\Sigma}|-\frac{1}{2} \sum_{i=1}^{K} \mathbf{u}_{i}^{\top} \boldsymbol{\Sigma}^{-1} \mathbf{u}_{i}
$$

The score for the complete data may be computed from the following expressions:

$$
\begin{aligned}
& \frac{\partial \log L_{c}}{\partial \boldsymbol{\beta}}=\sum_{i=1}^{K} \sum_{j=1}^{n_{i}} \mathbf{x}_{i j}^{\top} \boldsymbol{\Sigma}_{e}^{-1} \boldsymbol{\epsilon}_{i j} \\
& \frac{\partial \log L_{c}}{\partial \boldsymbol{\Sigma}}=-\frac{1}{2} \sum_{i=1}^{n}\left\{\operatorname{vec}\left(\left\{\boldsymbol{\Sigma}^{-1}\right\}^{\top}\right)-\left(\boldsymbol{\Sigma}^{-1} \otimes \boldsymbol{\Sigma}^{-1}\right)\left(\mathbf{u}_{i} \otimes \mathbf{u}_{i}\right)\right\} \\
& \frac{\partial \log L_{c}}{\partial \boldsymbol{\Sigma}_{e}}=-\frac{1}{2} \sum_{i=1}^{K} \sum_{j=1}^{n_{i}}\left\{\operatorname{vec}\left(\left\{\boldsymbol{\Sigma}_{e}^{-1}\right\}^{\top}\right)-\left(\boldsymbol{\Sigma}_{e}^{-1} \otimes \boldsymbol{\Sigma}_{e}^{-1}\right)\left(\boldsymbol{\epsilon}_{i j} \otimes \boldsymbol{\epsilon}_{i j}\right)\right\}
\end{aligned}
$$


To compute the MLEs, it is convenient to also observe the following:

$$
\begin{aligned}
& \frac{\partial \log L_{c}}{\partial \boldsymbol{\Sigma}^{-1}}=-\frac{1}{2} \sum_{i=1}^{n}\left[\operatorname{vec}\left(\{\boldsymbol{\Sigma}\}^{\top}\right)-\mathbf{u}_{i} \mathbf{u}_{i}^{\top}\right] \\
& \frac{\partial \log L_{c}}{\partial \boldsymbol{\Sigma}_{e}^{-1}}=-\frac{1}{2} \sum_{i=1}^{K} \sum_{j=1}^{n_{i}}\left[\operatorname{vec}\left(\left\{\boldsymbol{\Sigma}_{e}\right\}^{\top}\right)-\boldsymbol{\epsilon}_{i j} \boldsymbol{\epsilon}_{i j}^{\top}\right]
\end{aligned}
$$

Hence the MLE equations

$$
\begin{aligned}
\widehat{\boldsymbol{\Sigma}} & =\frac{1}{K} \sum_{i=1}^{K} \mathbf{u}_{i} \mathbf{u}_{i}^{\top} \\
\widehat{\boldsymbol{\beta}} & =\left(\sum_{i=1}^{K} \sum_{j=1}^{n_{i}} \mathbf{x}_{i j}^{\top} \widehat{\boldsymbol{\Sigma}}_{e}^{-1} \mathbf{x}_{i j}\right)^{-1}\left[\sum_{i=1}^{K} \sum_{j=1}^{n_{i}} \mathbf{x}_{i j}^{\top} \widehat{\boldsymbol{\Sigma}}_{e}^{-1}\left(\mathbf{y}_{i j}^{*}-\mathbf{z}_{i j} \mathbf{u}_{i}\right)\right] \\
\widehat{\boldsymbol{\Sigma}}_{e} & =\frac{1}{N} \sum_{i=1}^{K} \sum_{j=1}^{n_{i}} \boldsymbol{\epsilon}_{i j}(\widehat{\boldsymbol{\beta}}) \boldsymbol{\epsilon}_{i j}(\widehat{\boldsymbol{\beta}})^{\top}
\end{aligned}
$$

where $N=n_{1}+\ldots+n_{K}$.

The Fisher information matrix may be obtained from the following expressions:

$$
\begin{aligned}
& \frac{\partial^{2} \log L_{c}}{\partial \boldsymbol{\Sigma} \partial \boldsymbol{\Sigma}^{\top}}=\frac{1}{2} \sum_{i=1}^{K}\left\{\boldsymbol{\Sigma}^{-1} \otimes \boldsymbol{\Sigma}^{-1}-\right. \\
& {\left[\left\{\mathbf{K}_{s, s}\left(\boldsymbol{\Sigma}^{-1} \otimes \boldsymbol{\Sigma}^{-1}\right)\right\} \otimes\left\{\operatorname{vec}\left(\boldsymbol{\Sigma}^{-1}\right)\right\}^{\top}+\left\{\operatorname{vec}\left(\boldsymbol{\Sigma}^{-1}\right)\right\}^{\top} \otimes \boldsymbol{\Sigma}^{-1} \otimes \boldsymbol{\Sigma}^{-1}\right]\left(\mathbf{I}_{s} \otimes \mathbf{K}_{s, s} \otimes \mathbf{I}_{s}\right)} \\
& \left.\left[\operatorname{vec}\left(\mathbf{b}_{i} \mathbf{b}_{i}^{\top}\right) \otimes \mathbf{I}_{s^{2}}\right]\right\} . \\
& \qquad \frac{\partial^{2} \log L_{c}}{\partial \boldsymbol{\beta} \partial \boldsymbol{\beta}^{\top}}=-\sum_{i=1}^{K} \sum_{j=1}^{n_{i}} \mathbf{x}_{i j}^{\top} \boldsymbol{\Sigma}_{e}^{-1} \mathbf{x}_{i j} . \\
& \frac{\partial^{2} \log L_{c}}{\partial \boldsymbol{\Sigma}_{e} \partial \boldsymbol{\Sigma}_{e}^{\top}}=\frac{1}{2} \sum_{i=1}^{K} \sum_{j=1}^{n_{i}}\left\{\boldsymbol{\Sigma}_{e}^{-1} \otimes \boldsymbol{\Sigma}_{e}^{-1}-\right. \\
& {\left[\left\{\mathbf{K}_{r, r}\left(\boldsymbol{\Sigma}_{e}^{-1} \otimes \boldsymbol{\Sigma}_{e}^{-1}\right)\right\} \otimes\left\{\operatorname{vec}\left(\boldsymbol{\Sigma}_{e}^{-1}\right)\right\}^{\top}+\left\{\operatorname{vec}\left(\boldsymbol{\Sigma}_{e}^{-1}\right)\right\}^{\top} \otimes\left(\boldsymbol{\Sigma}_{e}^{-1} \otimes \boldsymbol{\Sigma}_{e}^{-1}\right)\right]\left(\mathbf{I}_{r} \otimes \mathbf{K}_{r, r} \otimes \mathbf{I}_{r}\right)} \\
& \left.\left[\operatorname{vec}\left(\boldsymbol{e}_{i j} \boldsymbol{e}_{i j}^{\top}\right) \otimes \mathbf{I}_{r}{ }^{\prime}\right]\right\} .
\end{aligned}
$$


where $\mathbf{K}_{s, s}$ and $\mathbf{K}_{r, r}$ are communtation matrices defined in Appendix A. Also

$$
\frac{\partial^{2} \log L_{c}}{\partial \boldsymbol{\Sigma}_{e} \partial \boldsymbol{\beta}_{e}^{\top}}=-\frac{1}{2}\left(\boldsymbol{\Sigma}_{e}^{-1} \otimes \boldsymbol{\Sigma}_{e}^{-1}\right)\left(\mathbf{K}_{r, r}+\mathbf{I}_{r^{2}}\right)\left(\sum_{i=1}^{K} \sum_{j=1}^{n_{i}} \mathbf{x}_{i j}^{\top} \otimes \boldsymbol{\epsilon}_{i j}\right)
$$

\subsubsection{Observed data}

For the observed data, the ML equations, score and observed information matrix are obtained from the complete expressions according to the missing information principle as described in Section 3.2.2. In this case the ML equations are as follows:

$$
\begin{aligned}
\widehat{\boldsymbol{\Sigma}} & =\frac{1}{K} \sum_{i=1}^{K} E\left[\mathbf{u}_{i} \mathbf{u}_{i}^{\top} \mid \mathbf{Y}=\mathbf{y}, \widehat{\boldsymbol{\psi}}\right] \\
\widehat{\boldsymbol{\beta}} & =\left(\sum_{i=1}^{K} \sum_{j=1}^{n_{i}} \mathbf{x}_{i j}^{\top} \widehat{\boldsymbol{\Sigma}}_{e}^{-1} \mathbf{x}_{i j}\right)^{-1}\left[\sum_{i=1}^{K} \sum_{j=1}^{n_{i}} \mathbf{x}_{i j}^{\top} \widehat{\boldsymbol{\Sigma}}_{e}^{-1} E\left[\mathbf{y}_{i j}^{*}-\mathbf{z}_{i j} \mathbf{u}_{i} \mid \mathbf{Y}=\mathbf{y}, \widehat{\boldsymbol{\psi}}\right]\right] . \\
\widehat{\boldsymbol{\Sigma}}_{e} & =\frac{1}{N} \sum_{i=1}^{K} \sum_{j=1}^{n_{i}} E\left[\boldsymbol{\epsilon}_{i j}(\widehat{\boldsymbol{\beta}}) \boldsymbol{\epsilon}_{i j}(\widehat{\boldsymbol{\beta}})^{\top} \mid \mathbf{Y}=\mathbf{y}, \widehat{\boldsymbol{\psi}}\right] .
\end{aligned}
$$

\subsection{Random effects according to a normal mixture}

The model of the previous section is easily extended to the case where the random effects distribution is an $m$ mixture of Gaussian. For each cluster $i$, consider $\mathbf{c}_{i}=$ $\left[c_{i 1} \ldots c_{i m}\right]^{\top}$ such that $c_{1}, \ldots, c_{n}$ are iid according to a multinomial distribution with parameters $(1, \mathbf{p})$ where $\mathbf{p}=\left(p_{1}, \ldots, p_{m}\right)$ is an $m$-tuple of probabilities adding up to 1. Conditional on $c_{i k}=1$, the random effect $\mathbf{u}_{i}$ is distributed according to $N\left(\boldsymbol{\mu}_{k}, \mathbf{D}\right)$ where $E\left[\mathbf{u}_{i}\right]=\mathbf{0}$. The need for zero-mean random effects, i.e. $E\left[\mathbf{u}_{i}\right]=\mathbf{0}$, imposes the constraint $p_{1} \boldsymbol{\mu}_{1}+\ldots+p_{m} \boldsymbol{\mu}_{m}=\mathbf{0}$ that either leads to a complicated expression for the complete data likelihood (where $\boldsymbol{\mu}_{m}$ is expressed as a function of the other means), or to a problem of constrained maximization involving lagrange multipliers. Instead it is more advantageous to re-parametrize the problem as suggested by S. Litiere et al. [16] as follows. 
- Define $\mathbf{v}_{i}=\mathbf{u}_{i}+\left[\begin{array}{l}\beta_{10} \\ \beta_{20}\end{array}\right]$, i.e. the sum of the random effect and the intercept terms.

- Define $\boldsymbol{\beta}^{\prime}$ as the vector of regression coefficients excluding the intercept terms.

In the new parametrization, $\mathbf{v}_{i}$ plays the role of $\mathbf{u}_{i}$ and $\boldsymbol{\beta}^{\prime}$ that of $\boldsymbol{\beta}$. This is equivalent to considering a model with null intercept but where random effects are allowed to have a non-zero mean. With this understanding, and to simplify the presentation, the same notation is used for the random effects and regression coefficients, i.e. $\mathbf{u}_{i}$ and $\boldsymbol{\beta}$. Let $\boldsymbol{\beta}_{0}=\left[\begin{array}{ll}\beta_{10} & \beta_{20}\end{array}\right]^{\top}$ denote the vector of intercept parameters. In the new parametrization, we have

$$
\boldsymbol{\beta}_{0}=E\left[\mathbf{u}_{i}\right]=\sum_{k=1}^{m} p_{k} \boldsymbol{\mu}_{k} .
$$

The variance $\boldsymbol{\Sigma}$ of the random effects has the expression

$$
\boldsymbol{\Sigma}=\mathbf{D}+\sum_{k=1}^{m} p_{k} \boldsymbol{\mu}_{k} \boldsymbol{\mu}_{k}^{\top}-\boldsymbol{\beta}_{0} \boldsymbol{\beta}_{0}^{\top}
$$

\subsubsection{Complete data}

Define $\mathbf{c}=\left[\begin{array}{llll}\mathbf{c}_{1} & \ldots & \mathbf{c}_{n}\end{array}\right]^{\top}$. In this case, the complete data is defined as $\left(\mathbf{Y}^{\star}, \mathbf{u}, \mathbf{c}\right)$ and $\boldsymbol{\psi}$ the vector of parameters includes $\boldsymbol{\beta}, \boldsymbol{\Sigma}_{e}, \mathbf{D}, p_{1}, \ldots, p_{m}, \boldsymbol{\mu}_{1}, \ldots, \boldsymbol{\mu}_{m}$. The log-likelihood is as follows:

$$
\begin{aligned}
\log L_{c}\left(\boldsymbol{\psi} ; \mathbf{u}, \mathbf{c}, \mathbf{y}^{*}\right)= & -\frac{1}{2} \sum_{i=1}^{K} \sum_{j=1}^{n_{i}} \log \left|\boldsymbol{\Sigma}_{e}\right|-\frac{1}{2} \sum_{i=1}^{K} \sum_{j=1}^{n_{i}} \boldsymbol{\epsilon}_{i j}^{\top} \boldsymbol{\Sigma}_{\boldsymbol{e}}^{-1} \boldsymbol{\epsilon}_{i j}-\frac{1}{2} \sum_{i=1}^{K} \sum_{k=1}^{m} c_{i k} \log |\mathbf{D}|- \\
& \frac{1}{2} \sum_{i=1}^{K} \sum_{k=1}^{m} c_{i k}\left(\mathbf{u}_{i}-\boldsymbol{\mu}_{k}\right)^{\top} \mathbf{D}^{-1}\left(\mathbf{u}_{i}-\boldsymbol{\mu}_{k}\right)+ \\
& \sum_{i=1}^{K}\left[\sum_{k=1}^{m-1} c_{i k} \log p_{k}+c_{i m} \log \left(1-p_{1}-\ldots-p_{m-1}\right)\right]
\end{aligned}
$$


The score equations are

$$
\begin{aligned}
& \frac{\partial \log L_{c}}{\partial \boldsymbol{\beta}}=\sum_{i=1}^{K} \sum_{j=1}^{n_{i}} \mathbf{x}_{i j}^{\top} \boldsymbol{\Sigma}_{e}^{-1} \boldsymbol{\epsilon}_{i j} . \\
& \frac{\partial \log L_{c}}{\partial \boldsymbol{\Sigma}_{e}}=-\frac{1}{2} \sum_{i=1}^{K} \sum_{j=1}^{n_{i}}\left\{\operatorname{vec}\left(\left\{\boldsymbol{\Sigma}_{e}^{-1}\right\}^{\top}\right)-\left(\boldsymbol{\Sigma}_{e}^{-1} \otimes \boldsymbol{\Sigma}_{e}^{-1}\right)\left(\boldsymbol{\epsilon}_{i j} \otimes \boldsymbol{\epsilon}_{i j}\right)\right\} \\
& \frac{\partial \log L_{c}}{\partial \mathbf{D}}=-\frac{1}{2} \sum_{i=1}^{K} \sum_{k=1}^{m} c_{i k}\left\{\operatorname{vec}\left(\left\{\mathbf{D}^{-1}\right\}^{\top}\right)-\left(\mathbf{D}^{-1} \otimes \mathbf{D}^{-1}\right)\left[\left(\mathbf{u}_{i}-\boldsymbol{\mu}_{k}\right) \otimes\left(\mathbf{u}_{i}-\boldsymbol{\mu}_{k}\right)\right]\right\} . \\
& \frac{\partial \log L_{c}}{\partial \boldsymbol{\mu}_{k}}=\sum_{i=1}^{K} c_{i k} \mathbf{D}^{-1}\left(\mathbf{u}_{i}-\boldsymbol{\mu}_{k}\right), k=1, \ldots, m . \\
& \frac{\partial \log L_{c}}{\partial p_{k}}=-\frac{1}{p_{m}}\left(\sum_{i=1}^{K} c_{i m}\right)+\frac{1}{p_{k}}\left(\sum_{i=1}^{K} c_{i k}\right), k=1, \ldots, m-1 .
\end{aligned}
$$

Also

$$
\frac{\partial \log L_{c}}{\partial \mathbf{D}^{-1}}=-\frac{1}{2} \sum_{i=1}^{K} \sum_{k=1}^{m} c_{i k}\left[\operatorname{vec}\left(\{\mathbf{D}\}^{\top}\right)-\left(\mathbf{u}_{i}-\boldsymbol{\mu}_{k}\right)\left(\mathbf{u}_{i}-\boldsymbol{\mu}_{k}\right)^{\top}\right]
$$

The information matrix may be obtained from the following equations.

$$
\begin{aligned}
& \frac{\partial^{2} \log L_{c}}{\partial \mathbf{D} \partial \mathbf{D}^{\top}}=\frac{1}{2} \sum_{i=1}^{K} \sum_{k=1}^{m} c_{i k}\left\{\mathbf{D}^{-1} \otimes \mathbf{D}^{-1}-\right. \\
& {\left[\left\{\mathbf{K}_{s, s}\left(\mathbf{D}^{-1} \otimes \mathbf{D}^{-1}\right)\right\} \otimes\left\{\operatorname{vec}\left(\mathbf{D}^{-1}\right)\right\}^{\top}+\left\{\operatorname{vec}\left(\mathbf{D}^{-1}\right)\right\}^{\top} \otimes \mathbf{D}^{-1} \otimes \mathbf{D}^{-1}\right]\left(\mathbf{I}_{s} \otimes \mathbf{K}_{s, s} \otimes \mathbf{I}_{s}\right)} \\
& \left.\left[\operatorname{vec}\left(\left(\mathbf{u}_{i}-\boldsymbol{\mu}_{k}\right)\left(\mathbf{u}_{i}-\boldsymbol{\mu}_{k}\right)^{\top}\right) \otimes \mathbf{I}_{s^{2}}\right]\right\} .
\end{aligned}
$$




$$
\begin{aligned}
& \frac{\partial^{2} \log L_{c}}{\partial \boldsymbol{\mu}_{k} \partial \boldsymbol{\mu}_{k}^{\top}}=-\sum_{i=1}^{K} c_{i k}\left(\mathbf{D}^{-1}\right)^{\top} . \\
& \frac{\partial^{2} \log L_{c}}{\partial \mathbf{D} \partial \boldsymbol{\mu}_{k}^{\top}}=-\left[\mathbf{D}^{-1} \otimes\left(\mathbf{D}^{\top}\right)^{-1}\right] \mathbf{K}_{s, s} \sum_{i=1}^{K} c_{i k}\left\{\mathbf{I}_{s} \otimes\left(\mathbf{u}_{i}-\boldsymbol{\mu}_{k}\right)\right\} . \\
& \frac{\partial^{2} \log L_{c}}{\partial p_{k}^{2}}=-\frac{1}{p_{m}^{2}}\left(\sum_{i=1}^{K} c_{i m}\right)-\frac{1}{p_{k}^{2}}\left(\sum_{i=1}^{K} c_{i k}\right), k=1, \ldots, m-1 . \\
& \frac{\partial^{2} \log L_{c}}{\partial p_{k} \partial p_{\ell}}=-\frac{1}{p_{m}^{2}}\left(\sum_{i=1}^{K} c_{i m}\right), 1 \leq k<\ell \leq m-1 .
\end{aligned}
$$

The other second order derivatives are the same as in the case of Gaussian random effects.

\subsubsection{Observed data}

The score for the observed data $\mathrm{Y}$ is given by the following equations

$$
\begin{aligned}
& \frac{\partial \log L(\boldsymbol{\psi} ; \mathbf{y})}{\partial \boldsymbol{\beta}}=\sum_{i=1}^{K} \sum_{j=1}^{n_{i}} \mathbf{x}_{i j}^{\top} \boldsymbol{\Sigma}_{e}^{-1} E\left[\boldsymbol{\epsilon}_{i j} \mid \mathbf{Y}=\mathbf{y}\right] \\
& \frac{\partial \log L(\boldsymbol{\psi} ; \mathbf{y})}{\partial \boldsymbol{\Sigma}_{e}}=-\frac{1}{2} \sum_{i=1}^{n} \sum_{j=1}^{n_{i}}\left\{\operatorname{vec}\left(\left\{\boldsymbol{\Sigma}_{e}^{-1}\right\}^{\top}\right)-\left(\boldsymbol{\Sigma}_{e}^{-1} \otimes \boldsymbol{\Sigma}_{e}^{-1}\right) E\left[\boldsymbol{\epsilon}_{i j} \otimes \boldsymbol{\epsilon}_{i j} \mid \mathbf{Y}=\mathbf{y}\right]\right\}
\end{aligned}
$$

$$
\begin{aligned}
& \frac{\partial \log L(\boldsymbol{\psi} ; \mathbf{y})}{\partial \mathbf{D}}= \\
& -\frac{1}{2} \sum_{i=1}^{K} \sum_{k=1}^{m} E\left[c_{i k}\left\{\operatorname{vec}\left(\left\{\mathbf{D}^{-1}\right\}^{\top}\right)-\left(\mathbf{D}^{-1} \otimes \mathbf{D}^{-1}\right)\left(\left(\mathbf{u}_{i}-\boldsymbol{\mu}_{k}\right) \otimes\left(\mathbf{u}_{i}-\boldsymbol{\mu}_{k}\right)\right)\right\} \mid \mathbf{Y}=\mathbf{y}\right]
\end{aligned}
$$

$$
\begin{aligned}
& \frac{\partial \log L(\boldsymbol{\psi} ; \mathbf{y})}{\partial \boldsymbol{\mu}_{k}}=\sum_{i=1}^{K} \mathbf{D}^{-1} E\left[c_{i k}\left(\mathbf{u}_{i}-\boldsymbol{\mu}_{k}\right) \mid \mathbf{Y}=\mathbf{y}\right], k=1, \ldots, m \\
& \frac{\partial \log L(\boldsymbol{\psi} ; \mathbf{y})}{\partial p_{k}}=-\frac{1}{p_{m}}\left(\sum_{i=1}^{K} E\left[c_{i m} \mid \mathbf{Y}=\mathbf{y}\right]\right)+\frac{1}{p_{k}}\left(\sum_{i=1}^{K} E\left[c_{i k} \mid \mathbf{Y}=\mathbf{y}\right]\right), k=1, \ldots, m-1
\end{aligned}
$$


ML equations for the observed data are as follows:

$$
\frac{\partial \log L}{\partial \boldsymbol{\beta}}=\mathbf{0}, \quad \frac{\partial \log L}{\partial \boldsymbol{\Sigma}_{e}^{-1}}=\mathbf{0}, \quad \frac{\partial \log L}{\partial \mathbf{D}^{-1}}=\mathbf{0}, \quad \frac{\partial \log L}{\partial \boldsymbol{\mu}_{k}}=\mathbf{0}, \quad \frac{\partial \log L}{\partial p_{k}}=0
$$

Hence the following MLEs are obtained.

$$
\begin{aligned}
& \widehat{\boldsymbol{\beta}}=\left(\sum_{i=1}^{K} \sum_{j=1}^{n_{i}} \mathbf{x}_{i j}^{\top} \widehat{\boldsymbol{\Sigma}}_{e}^{-1} \mathbf{x}_{i j}\right)^{-1}\left[\sum_{i=1}^{K} \sum_{j=1}^{n_{i}} \mathbf{x}_{i j}^{\top} \widehat{\boldsymbol{\Sigma}}_{e}^{-1} E\left[\left(\mathbf{y}_{i j}^{*}-\mathbf{z}_{i j} \mathbf{u}_{i}\right) \mid \mathbf{Y}=\mathbf{y}, \widehat{\boldsymbol{\psi}}\right]\right] \\
& \widehat{\boldsymbol{\Sigma}}_{\boldsymbol{e}}=\frac{1}{N} \sum_{i=1}^{K} \sum_{j=1}^{n_{i}} E\left[\boldsymbol{\epsilon}_{i j}(\widehat{\boldsymbol{\beta}}) \boldsymbol{\epsilon}_{i j}(\widehat{\boldsymbol{\beta}})^{\top} \mid \mathbf{Y}=\mathbf{y}, \widehat{\boldsymbol{\psi}}\right] \\
& \widehat{p}_{m}=\left(1+\sum_{k=1}^{m-1} \frac{\sum_{i=1}^{K} E\left[c_{i k} \mid \mathbf{Y}=\mathbf{y}, \widehat{\boldsymbol{\psi}}\right]}{\sum_{i=1}^{K} E\left[c_{i m} \mid \mathbf{Y}=\mathbf{y}, \widehat{\boldsymbol{\psi}}\right]}\right)^{-1} \\
& \widehat{p}_{k}=\widehat{p}_{m}\left(\frac{\sum_{i=1}^{K} E\left[c_{i k} \mid \mathbf{Y}=\mathbf{y}, \widehat{\boldsymbol{\psi}}\right]}{\sum_{i=1}^{K} E\left[c_{i m} \mid \mathbf{Y}=\mathbf{y}, \widehat{\boldsymbol{\psi}}\right]}\right), k=1, \ldots, m-1 \\
& \widehat{\mathbf{D}}=\left(\sum_{i=1}^{K} \sum_{k=1}^{m} E\left[c_{i k} \mid \mathbf{Y}=\mathbf{y}, \widehat{\boldsymbol{\psi}}\right]\right)^{-1}\left(\sum_{i=1}^{K} \sum_{k=1}^{m} E\left[c_{i k}\left(\mathbf{u}_{i}-\widehat{\boldsymbol{\mu}}_{k}\right)\left(\mathbf{u}_{i}-\widehat{\boldsymbol{\mu}}_{k}\right)^{\top} \mid \mathbf{Y}=\mathbf{y}, \widehat{\boldsymbol{\psi}}\right]\right) \\
& \widehat{\boldsymbol{\mu}}_{k}=\left(\sum_{i=1}^{K} E\left[c_{i k} \mid \mathbf{Y}=\mathbf{y}, \widehat{\boldsymbol{\psi}}\right]\right)^{-1}\left(\sum_{i=1}^{K} E\left[c_{i k} \mathbf{u}_{i} \mid \mathbf{Y}=\mathbf{y}, \widehat{\boldsymbol{\psi}}\right]\right)
\end{aligned}
$$

The observed information matrix is obtained from the results for the complete data through Louis's formula given in Section 3.2.2.

The above ML equations are fixed-point equations since by definition $\hat{\psi}$ is a function of all the parameters including $\hat{\boldsymbol{\beta}}, \hat{\boldsymbol{\Sigma}}_{e}, \mathrm{D}, \hat{\boldsymbol{\mu}}_{1}, \ldots, \hat{\boldsymbol{\mu}}_{m}$ and $\hat{p}_{1}, \ldots, \hat{p}_{m}$. Therefore, they can only be solved iteratively. 


\section{Chapter 5}

\section{SAECM algorithms}

The SAECM directly follows from the ML equations described in the previous chapter. For some function $g\left(\mathbf{y}^{*}, \mathbf{u}, \mathbf{c}\right)$ of the complete data, it is convenient to denote by $\hat{E}_{r+1}\left[g\left(\mathbf{y}^{*}, \mathbf{u}, \mathbf{c}\right) \mid \mathbf{Y}=\mathbf{y}, \boldsymbol{\psi}^{(r)}\right]$, the estimate of $E\left[g\left(\mathbf{y}^{*}, \mathbf{u}, \mathbf{c}\right) \mid \mathbf{Y}=\mathbf{y}, \boldsymbol{\psi}^{(r)}\right]$ obtained in the $r+1$-th iteration of the SA procedure. The estimate is computed iteratively according to the following equations

$$
\begin{aligned}
\hat{E}_{1}\left[g\left(\mathbf{y}^{*}, \mathbf{u}, \mathbf{c}\right) \mid \mathbf{Y}=\mathbf{y}, \boldsymbol{\psi}^{(0)}\right]= & \frac{1}{S} \sum_{s=1}^{S} g\left(\mathbf{y}_{s}^{*(1)}, \mathbf{b}_{s}^{(1)}, \mathbf{c}_{s}^{(1)}\right) \\
\hat{E}_{r+1}\left[g\left(\mathbf{y}^{*}, \mathbf{u}, \mathbf{c}\right) \mid \mathbf{Y}=\mathbf{y}, \boldsymbol{\psi}^{(r)}\right]= & \left(1-w_{r+1}\right) \hat{E}_{r}\left[g\left(\mathbf{y}^{*}, \mathbf{u}, \mathbf{c}\right) \mid \mathbf{Y}=\mathbf{y}, \boldsymbol{\psi}^{(r-1)}\right]+ \\
& w_{r+1} g\left(\mathbf{y}^{*(r+1)}, \mathbf{b}^{(r+1)}, \mathbf{c}^{(r+1)}\right), r \geq 1
\end{aligned}
$$

where

- $\left\{w_{r}\right\}_{r \geq 1}$ is a selected sequence of nonincreasing positive weights such that $\sum_{r=1}^{\infty} w_{r}=\infty$ and $\sum_{r=1}^{\infty} w_{r}^{2}<\infty$. Typically $w_{r}=r^{\alpha}$ where $\alpha \in(1 / 2,1]$.

- $\left(\mathbf{y}_{s}^{*(1)}, \mathbf{b}_{s}^{(1)}, \mathbf{c}_{s}^{(1)}\right)$ are iid according to the conditional distribution of the complete data given the observed data and assuming that the parameter vector is $\boldsymbol{\psi}^{(0)}$ (the inital parameter vector).

- $\left(\mathbf{y}^{*(r+1)}, \mathbf{b}^{(r+1)}, \mathbf{c}^{(r+1)}\right)$ is drawn from the conditional distribution of the complete 
data given the observed data, and assuming that the parameter vector is $\psi^{(r)}$.

For some functions $a$ and $d$ of the parameters define

$$
\begin{aligned}
\hat{E}_{r+1}\left[a(\boldsymbol{\psi})+d(\boldsymbol{\psi}) g\left(\mathbf{y}^{*}, \mathbf{u}, \mathbf{c}\right) \mid \mathbf{Y}=\mathbf{y}, \boldsymbol{\psi}^{(r)}\right]:= & a\left(\boldsymbol{\psi}^{(r)}\right)+ \\
& d\left(\boldsymbol{\psi}^{(r)}\right) \hat{E}_{r+1}\left[g\left(\mathbf{y}^{*}, \mathbf{u}, \mathbf{c}\right) \mid \mathbf{Y}=\mathbf{y}, \boldsymbol{\psi}^{(r)}\right]
\end{aligned}
$$

\subsection{Random effects with a normal distribution}

When random effects are normal, the ML equations may be solved using the SAECM procedure proposed by R. Gueorguieva and A. Agresti [20], which we now describe. At each iteration, it generates data from the conditional distribution of the complete data given the observed data using a Gibbs sampler. The E step, M step and Gibbs sampler are subsequently described in details.

\subsubsection{E step}

In the $\mathrm{E}$ step, estimates of the following conditional expectations are updated:

$$
\begin{aligned}
& -E\left[\mathbf{u}_{i} \mid \mathbf{Y}=\mathbf{y}, \boldsymbol{\psi}\right] \\
& -E\left[\mathbf{u}_{i} \mathbf{u}_{i}^{\top} \mid \mathbf{Y}=\mathbf{y}, \boldsymbol{\psi}\right] \\
& -E\left[\mathbf{y}_{i j}^{*}-\mathbf{z}_{i j} \mathbf{u}_{i} \mid \mathbf{Y}=\mathbf{y}, \boldsymbol{\psi}\right] \\
& -E\left[\left(\mathbf{y}_{i j}^{*}-\mathbf{z}_{i j} \mathbf{u}_{i}\right)\left(\mathbf{y}_{i j}^{*}-\mathbf{z}_{i j} \mathbf{u}_{i}\right)^{\top} \mid \mathbf{Y}=\mathbf{y}, \boldsymbol{\psi}\right]
\end{aligned}
$$


These updates are done according to the following equations:

$$
\begin{aligned}
& \hat{E}_{r+1}\left[\mathbf{u}_{i} \mid \mathbf{Y}=\mathbf{y}, \boldsymbol{\psi}^{(r)}\right]= w_{r+1} \mathbf{u}_{i}^{(r+1)}+ \\
&\left(1-w_{r+1}\right) \hat{E}_{r}\left[\mathbf{u}_{i} \mid \mathbf{Y}=\mathbf{y}, \boldsymbol{\psi}^{(r-1)}\right] \\
& \hat{E}_{r+1}\left[\mathbf{u}_{i} \mathbf{u}_{i}^{\top} \mid \mathbf{Y}=\mathbf{y}, \boldsymbol{\psi}^{(r)}\right]= w_{r+1} \mathbf{u}_{i}^{(r+1)}\left\{\mathbf{u}_{i}^{(r+1)}\right\}^{\top}+ \\
&\left(1-w_{r+1}\right) \hat{E}_{r}\left[\mathbf{u}_{i} \mathbf{u}_{i}^{\top} \mid \mathbf{Y}=\mathbf{y}, \boldsymbol{\psi}^{(r-1)}\right] \\
& \hat{E}_{r+1}\left[\mathbf{y}_{i j}^{*}-\mathbf{z}_{i j} \mathbf{u}_{i} \mid \mathbf{Y}=\mathbf{y}, \boldsymbol{\psi}^{(r)}\right]= w_{r+1}\left[\mathbf{y}_{i j}^{*(r+1)}-\mathbf{z}_{i j} \mathbf{u}_{i}^{(r+1)}\right]+ \\
&\left(1-w_{r+1}\right) \hat{E}_{r}\left[\mathbf{y}_{i j}^{*}-\mathbf{z}_{i j} \mathbf{u}_{i} \mid \mathbf{Y}=\mathbf{y}, \boldsymbol{\psi}^{(r-1)}\right] \\
& \hat{E}_{r+1}\left[\left(\mathbf{y}_{i j}^{*}-\mathbf{z}_{i j} \mathbf{u}_{i}\right)\left(\mathbf{y}_{i j}^{*}-\mathbf{z}_{i j} \mathbf{u}_{i}\right)^{\top} \mid \mathbf{Y}=\mathbf{y}, \boldsymbol{\psi}^{(r)}\right]= \\
& w_{r+1}\left[\mathbf{y}_{i j}^{*(r+1)}-\mathbf{z}_{i j} \mathbf{u}_{i}^{(r+1)}\right]\left[\mathbf{y}_{i j}^{*(r+1)}-\mathbf{z}_{i j} \mathbf{u}_{i}^{(r+1)}\right]^{\top}+ \\
&\left(1-w_{r+1}\right) \hat{E}_{r}\left[\left(\mathbf{y}_{i j}^{*}-\mathbf{z}_{i j} \mathbf{u}_{i}\right)\left(\mathbf{y}_{i j}^{*}-\mathbf{z}_{i j} \mathbf{u}_{i}\right)^{\top} \mid \mathbf{Y}=\mathbf{y}, \boldsymbol{\psi}^{(r-1)}\right]
\end{aligned}
$$

\subsubsection{M step}

In the $\mathrm{M}$ step the parameter estimates are updated as follows:

$$
\begin{aligned}
& \boldsymbol{\Sigma}^{(r+1)}=\frac{1}{K} \sum_{i=1}^{K} \hat{E}_{r+1}\left[\mathbf{u}_{i} \mathbf{u}_{i}^{\top} \mid \mathbf{Y}=\mathbf{y}, \boldsymbol{\psi}^{(r)}\right] \\
& \boldsymbol{\Sigma}_{e}^{(r+1)}=\frac{1}{N} \sum_{i=1}^{K} \sum_{j=1}^{n_{i}} \hat{E}_{r+1}\left[\mathbf{e}_{i j}\left(\boldsymbol{\beta}^{(r)}\right) \mathbf{e}_{i j}\left(\boldsymbol{\beta}^{(r)}\right)^{\top} \mid \mathbf{Y}=\mathbf{y}, \boldsymbol{\psi}^{(r)}\right] \\
& \boldsymbol{\beta}^{(r+1)}=\left(\sum_{i=1}^{K} \sum_{j=1}^{n_{i}} \mathbf{x}_{i j}^{\top}\left\{\boldsymbol{\Sigma}_{e}^{(r+1)}\right\}^{-1} \mathbf{x}_{i j}\right)^{-1}\left(\sum_{i=1}^{K} \sum_{j=1}^{n_{i}} \mathbf{x}_{i j}^{\top}\left\{\boldsymbol{\Sigma}_{e}^{(r+1)}\right\}^{-1} \hat{E}_{r+1}\left[\left(\mathbf{y}_{i j}^{*}-\mathbf{z}_{i j} \mathbf{u}_{i}\right) \mid \mathbf{Y}=\mathbf{y}, \boldsymbol{\psi}^{(r)}\right]\right)
\end{aligned}
$$




\subsubsection{Gibbs sampler}

The goal is to generate samples from the conditional distribution of $\left(\mathbf{u}_{i}, \mathbf{Y}_{i}^{*}\right)$ given the observed data $\mathbf{Y}_{i}=\mathbf{y}_{i}$. The vector $\mathbf{Y}_{i}^{*}$ has a truncated normal distribution given the observed data $\mathbf{Y}_{i}=\mathbf{y}_{i}$, while the random effects $\mathbf{u}_{i}$ have a normal distribution given $\mathbf{Y}_{i}^{*}$. Let $\mathbf{u}_{i}^{(r)}$ and $\mathbf{y}_{i}^{*(r)}$ denote the samples drawn from the conditional distribution of the complete data at iteration $r$. The sampler operates according to the following procedure:

a) Initialization: For each cluster $i$, select any $\mathbf{u}_{i}^{(0)}$ and $\mathbf{y}^{*(0)}$ compatible with the observed data $\mathbf{y}_{i}$

b) Iteration $r$ : for each cluster $i$

1. Sampling of the latent responses: Use a Gibbs sampler for truncated normal distributions to draw $\mathbf{y}_{i}^{*(r)}$ based on $\mathbf{y}_{i}^{*(r-1)}$ and the current parameter values $\boldsymbol{\psi}^{(r-1)}$

2. Sampling random effects: Select $\mathbf{u}_{i}^{(r)}$ based on the conditional distribution of the random effects given $\mathbf{Y}_{i}^{*}=\mathbf{y}_{i}^{*(r)}$ and the current parameter values $\psi^{(r-1)}$

\section{Sampling from a truncated normal distribution}

A generic Gibbs sampler is constructed as follows. Let $\mathbf{Y}^{*}=\left[\begin{array}{c}\mathbf{Y}_{1}^{*} \\ \mathbf{Y}_{2}^{*}\end{array}\right]$ denote a normal random vector, where $\mathbf{Y}_{1}^{*}$ and $\mathbf{Y}_{2}^{*}$ are respectively $p \times 1$ and $q \times 1$ random vectors. For a $q$ dimensional vector $\mathbf{v}$, and $i=1, \ldots, q$, define $\mathbf{v}_{(i)}=\left(v_{1}, \ldots, v_{i-1}, v_{i+1}, \ldots, v_{q}\right)$, the vector of all coordinates of $\mathbf{v}$ except the $i$-th one. Finally define $\mathbf{Y}=\left[\begin{array}{c}\mathbf{Y}_{1} \\ \\ \mathbf{Y}_{2}\end{array}\right]$, where 
$\mathbf{Y}_{1}=\mathbf{Y}_{1}^{*}$ and $Y_{2 i}=I\left\{Y_{2 i}^{*}>0\right\}$ for $i=1, \ldots, q$. For $i=1, \ldots, q$,

$$
\operatorname{Pr}\left(Y_{2 i}^{*(r+1)}=y_{2 i}^{*(r+1)}\right)=\operatorname{Pr}\left(Y_{2 i}^{*}=y_{2 i}^{*(r+1)} \mid \mathbf{Y}=\mathbf{y}, \mathbf{Y}_{2(i)}^{*}=\mathbf{y}_{2(i)}^{*(r+1, i+1)}\right)
$$

where $\mathbf{y}_{2(i)}^{*(r+1, i+1)}=\mathbf{y}_{2(i)}^{*(r+1,(i-1)+1)}$.

At the $r$-th iteration, $\mathbf{Y}_{2}^{*(r)}$ is generated. When $r$ becomes large, $\mathbf{Y}_{2}^{*(r)}$ converges to the desired conditional distribution.

Detailed expressions for the conditional probabilities are as follows. Consider $\mathbf{y}$ and $\mathbf{y}_{2}^{*}$ such that $\{\mathbf{Y}=\mathbf{y}\} \cap\left\{\mathbf{Y}_{2}^{*}=\mathbf{y}_{2}^{*}\right\} \neq \varnothing$, and let $\mathbf{y}^{*}=\left[\begin{array}{l}\mathbf{y}_{1} \\ \mathbf{y}_{2}^{*}\end{array}\right]$. For $i=1, \ldots, q$, let $\mu_{2 i}\left(\mathbf{y}_{1}^{*}, \mathbf{y}_{2(i)}^{*}\right)$ and $\sigma_{2 i}\left(\mathbf{y}_{1}^{*}, \mathbf{y}_{2(i)}^{*}\right)$ respectively denote the conditional mean and variance of $Y_{2 i}^{*}$ given $\mathbf{Y}_{1}^{*}=\mathbf{y}_{1}^{*}, \quad \mathbf{Y}_{2(i)}^{*}=\mathbf{y}_{2(i)}^{*}$. Also let $\Phi$ denote the normal cdf.

$$
\begin{gathered}
\operatorname{Pr}\left(Y_{2 i}^{*}=y_{2 i}^{*} \mid \mathbf{Y}=\mathbf{y}, \mathbf{Y}_{2(i)}^{*}=\mathbf{y}_{2(i)}^{*}\right)=I\left\{y_{2 i}^{*}>0\right\} \frac{\Phi\left(\frac{\mathbf{y}_{2 i}^{*}-\mu_{2 i}}{\sigma_{2 i}}\right)-\Phi\left(-\frac{\mu_{2 i}}{\sigma_{2 i}}\right)}{1-\Phi\left(-\frac{\mu_{2 i}}{\sigma_{2 i}}\right)}+ \\
I\left\{y_{2 i}^{*} \leq 0\right\} \frac{\Phi\left(\frac{\mathbf{y}_{2 i}^{*}-\mu_{2 i}}{\sigma_{2 i}}\right)}{\Phi\left(-\frac{\mu_{2 i}}{\sigma_{2 i}}\right)} .
\end{gathered}
$$

\section{Sampling of random effects}

The vectors $\mathbf{Y}_{i}^{*}$ and $\mathbf{u}_{i}$ have a joint normal distribution as follows:

$$
\left(\begin{array}{c}
\mathbf{Y}_{i}^{*} \\
\mathbf{u}_{i}
\end{array}\right) \sim N\left(\left(\begin{array}{c}
\mathbf{X}_{i} \boldsymbol{\beta} \\
\mathbf{0}
\end{array}\right),\left(\begin{array}{rr}
\mathbf{Z}_{i} \boldsymbol{\Sigma} \mathbf{Z}_{i}^{\top}+\boldsymbol{\Sigma}_{E_{i}} & \mathbf{Z}_{i} \boldsymbol{\Sigma} \\
\mathbf{\Sigma} \mathbf{Z}_{i}^{\top} & \boldsymbol{\Sigma}
\end{array}\right)\right)
$$


where

$$
\mathbf{X}_{i}=\left[\begin{array}{c}
\mathbf{x}_{i 1} \\
\vdots \\
\mathbf{x}_{i n_{i}}
\end{array}\right], \quad \mathbf{Z}_{i}=\left[\begin{array}{c}
\mathbf{z}_{i 1} \\
\vdots \\
\mathbf{z}_{i n_{i}}
\end{array}\right], \quad \mathbf{Y}_{i}^{*}=\left[\begin{array}{c}
\mathbf{Y}_{i 1}^{*} \\
\vdots \\
\mathbf{Y}_{i n_{i}}^{*}
\end{array}\right]
$$

Therefore

$$
\mathbf{u}_{i} \mid \mathbf{y}_{i}^{*} \sim N\left(\boldsymbol{\Sigma}_{B_{i}}\left(\mathbf{y}_{i}^{*}-\mathbf{X}_{i} \boldsymbol{\beta}\right), \boldsymbol{\Sigma}-\boldsymbol{\Sigma}_{B_{i}} \mathbf{Z}_{i} \boldsymbol{\Sigma}\right)
$$

where

$$
\begin{gathered}
\boldsymbol{\Sigma}_{E_{i}}=\boldsymbol{\Sigma}_{e} \otimes \boldsymbol{I}_{n_{i}}=\left[\begin{array}{ccc}
\boldsymbol{\Sigma}_{e} & \ldots & 0 \\
\vdots & \ddots & \vdots \\
0 & \ldots & \boldsymbol{\Sigma}_{e}
\end{array}\right] . \\
\boldsymbol{\Sigma}_{B_{i}}=\boldsymbol{\Sigma} \mathbf{Z}_{i}^{\top}\left(\mathbf{Z}_{i} \boldsymbol{\Sigma} \mathbf{Z}_{i}^{\top}+\boldsymbol{\Sigma}_{E_{i}}\right)^{-1} .
\end{gathered}
$$

\subsection{Random effects following a normal mixture}

An extended SAECM algorithm is presented for random effects that follow a normal mixture. Although some of the equations look similar to the case of normal random effects, the parametrization of Section 4.2 is assumed.

\subsubsection{E step}

In the $\mathrm{E}$ step, the complete data is drawn from its conditional distribution given the observed data and estimates of the following conditional expectations are updated:

$$
\begin{aligned}
& -E\left[c_{i k} \mid \mathbf{Y}=\mathbf{y}, \boldsymbol{\psi}\right] \\
& -E\left[c_{i k} \mathbf{u}_{i} \mid \mathbf{Y}=\mathbf{y}, \boldsymbol{\psi}\right]
\end{aligned}
$$




$$
\begin{aligned}
& -E\left[c_{i k} \mathbf{u}_{i} \mathbf{u}_{i}^{\top} \mid \mathbf{Y}=\mathbf{y}, \boldsymbol{\psi}\right] \\
& -E\left[\mathbf{y}_{i j}^{*}-\mathbf{z}_{i j} \mathbf{u}_{i} \mid \mathbf{Y}=\mathbf{y}, \boldsymbol{\psi}\right] \\
& -E\left[\left(\mathbf{y}_{i j}^{*}-\mathbf{z}_{i j} \mathbf{u}_{i}\right)\left(\mathbf{y}_{i j}^{*}-\mathbf{z}_{i j} \mathbf{u}_{i}\right)^{\top} \mid \mathbf{Y}=\mathbf{y}, \boldsymbol{\psi}\right]
\end{aligned}
$$

These updates are done according to the following equations:

$$
\begin{aligned}
& \hat{E}_{r+1}\left[c_{i k} \mid \mathbf{Y}=\mathbf{y}, \boldsymbol{\psi}^{(r)}\right]=w_{r+1} c_{i k}^{(r+1)}+\left(1-w_{r+1}\right) \hat{E}_{r}\left[c_{i k} \mid \mathbf{Y}=\mathbf{y}, \boldsymbol{\psi}^{(r-1)}\right] \\
& \hat{E}_{r+1}\left[c_{i k} \mathbf{u}_{i} \mid \mathbf{Y}=\mathbf{y}, \boldsymbol{\psi}^{(r)}\right]=w_{r+1} c_{i k}^{(r+1)} \mathbf{u}_{i}^{(r+1)}+ \\
& \left(1-w_{r+1}\right) \hat{E}_{r}\left[c_{i k} \mathbf{u}_{i} \mid \mathbf{Y}=\mathbf{y}, \boldsymbol{\psi}^{(r-1)}\right] \\
& \hat{E}_{r+1}\left[c_{i k} \mathbf{u}_{i} \mathbf{u}_{i}^{\top} \mid \mathbf{Y}=\mathbf{y}, \boldsymbol{\psi}^{(r)}\right]=w_{r+1} c_{i k}^{(r+1)} \mathbf{u}_{i}^{(r+1)}\left\{\mathbf{u}_{i}^{(r+1)}\right\}^{\top}+ \\
& \left(1-w_{r+1}\right) \hat{E}_{r}\left[c_{i k} \mathbf{u}_{i} \mathbf{u}_{i}^{\top} \mid \mathbf{Y}=\mathbf{y}, \boldsymbol{\psi}^{(r-1)}\right] \\
& \hat{E}_{r+1}\left[\mathbf{y}_{i j}^{*}-\mathbf{z}_{i j} \mathbf{u}_{i} \mid \mathbf{Y}=\mathbf{y}, \boldsymbol{\psi}^{(r)}\right]=w_{r+1}\left[\mathbf{y}_{i j}^{*(r+1)}-\mathbf{z}_{i j} \mathbf{u}_{i}^{(r+1)}\right]+ \\
& \left(1-w_{r+1}\right) \hat{E}_{r}\left[\mathbf{y}_{i j}^{*}-\mathbf{z}_{i j} \mathbf{u}_{i} \mid \mathbf{Y}=\mathbf{y}, \boldsymbol{\psi}^{(r-1)}\right] \\
& \hat{E}_{r+1}\left[\left(\mathbf{y}_{i j}^{*}-\mathbf{z}_{i j} \mathbf{u}_{i}\right)\left(\mathbf{y}_{i j}^{*}-\mathbf{z}_{i j} \mathbf{u}_{i}\right)^{\top} \mid \mathbf{Y}=\mathbf{y}, \boldsymbol{\psi}^{(r)}\right]= \\
& w_{r+1}\left[\mathbf{y}_{i j}^{*(r+1)}-\mathbf{z}_{i j} \mathbf{u}_{i}^{(r+1)}\right]\left[\mathbf{y}_{i j}^{*(r+1)}-\mathbf{z}_{i j} \mathbf{u}_{i}^{(r+1)}\right]^{\top}+ \\
& \left(1-w_{r+1}\right) \hat{E}_{r}\left[\left(\mathbf{y}_{i j}^{*}-\mathbf{z}_{i j} \mathbf{u}_{i}\right)\left(\mathbf{y}_{i j}^{*}-\mathbf{z}_{i j} \mathbf{u}_{i}\right)^{\top} \mid \mathbf{Y}=\mathbf{y}, \boldsymbol{\psi}^{(r-1)}\right] \\
& \hat{E}_{r+1}\left[c_{i k}\left(\mathbf{u}_{i}-\boldsymbol{\mu}_{k}^{(r+1)}\right)\left(\mathbf{u}_{i}-\boldsymbol{\mu}_{k}^{(r+1)}\right)^{\top} \mid \mathbf{Y}=\mathbf{y}, \boldsymbol{\psi}^{(r)}\right]= \\
& \hat{E}_{r+1}\left[c_{i k} \mathbf{u}_{i} \mathbf{u}_{i}^{\top} \mid \mathbf{Y}=\mathbf{y}, \boldsymbol{\psi}^{(r)}\right]+ \\
& \hat{E}_{r+1}\left[c_{i k} \mid \mathbf{Y}=\mathbf{y}, \boldsymbol{\psi}^{(r)}\right] \boldsymbol{\mu}_{k}^{(r+1)}\left\{\boldsymbol{\mu}_{k}^{(r+1)}\right\}^{\top}-\hat{E}_{r+1}\left[c_{i k} \mathbf{u}_{i} \mid \mathbf{Y}=\mathbf{y}, \boldsymbol{\psi}^{(r)}\right]\left\{\boldsymbol{\mu}_{k}^{(r+1)}\right\}^{\top}- \\
& \boldsymbol{\mu}_{k}^{(r+1)} \hat{E}_{r+1}\left[c_{i k} \mathbf{u}_{i} \mid \mathbf{Y}=\mathbf{y}, \boldsymbol{\psi}^{(r)}\right]^{\top}
\end{aligned}
$$




$$
\begin{aligned}
& \hat{E}_{r+1}\left[\mathbf{e}_{i j}\left(\boldsymbol{\beta}^{(r)}\right) \mathbf{e}_{i j}\left(\boldsymbol{\beta}^{(r)}\right)^{\top} \mid \mathbf{Y}=\mathbf{y}, \boldsymbol{\psi}^{(r)}\right]=\hat{E}_{r+1}\left[\left(\mathbf{y}_{i j}^{*}-\mathbf{z}_{i j} \mathbf{u}_{i}\right)\left(\mathbf{y}_{i j}^{*}-\mathbf{z}_{i j} \mathbf{u}_{i}\right)^{\top} \mid \mathbf{Y}=\mathbf{y}, \boldsymbol{\psi}^{(r)}\right]+ \\
& \mathbf{x}_{i j} \boldsymbol{\beta}^{(r)}\left\{\boldsymbol{\beta}^{(r)}\right\}^{\top} \mathbf{x}_{i j}^{\top}-\hat{E}_{r+1}\left[\left(\mathbf{y}_{i j}^{*}-\mathbf{z}_{i j} \mathbf{u}_{i}\right) \mid \mathbf{Y}=\mathbf{y}, \boldsymbol{\psi}^{(r)}\right]\left\{\boldsymbol{\beta}^{(r)}\right\}^{\top} \mathbf{x}_{i j}^{\top}- \\
& \mathbf{x}_{i j} \boldsymbol{\beta}^{(r)} \hat{E}_{r+1}\left[\left(\mathbf{y}_{i j}^{*}-\mathbf{z}_{i j} \mathbf{u}_{i}\right) \mid \mathbf{Y}=\mathbf{y}, \boldsymbol{\psi}^{(r)}\right]^{\top}
\end{aligned}
$$

\subsubsection{M step}

In the $M$ step the parameter estimates are updated as follows:

$$
\begin{aligned}
p_{m}^{(r+1)} & =\left(1+\sum_{k=1}^{m-1} \frac{\sum_{i=1}^{K} \hat{E}_{r+1}\left[c_{i k} \mid \mathbf{Y}=\mathbf{y}, \boldsymbol{\psi}^{(r)}\right]}{\sum_{i=1}^{K} \hat{E}_{r+1}\left[c_{i m} \mid \mathbf{Y}=\mathbf{y}, \boldsymbol{\psi}^{(r)}\right]}\right)^{-\mathbf{1}} \\
p_{k}^{(r+1)}= & p_{m}^{(r+1)}\left(\frac{\sum_{i=1}^{K} \hat{E}_{r+1}\left[c_{i k} \mid \mathbf{Y}=\mathbf{y}, \boldsymbol{\psi}^{(r)}\right]}{\sum_{i=1}^{K} \hat{E}_{r+1}\left[c_{i m} \mid \mathbf{Y}=\mathbf{y}, \boldsymbol{\psi}^{(r)}\right]}\right), k=1, \ldots, m-1 \\
\mathbf{D}^{(r+1)}= & \frac{\sum_{i=1}^{K} \sum_{k=1}^{m} \hat{E}_{r+1}\left[c_{i k}\left(\mathbf{u}_{i}-\boldsymbol{\mu}_{k}^{(r+1)}\right)\left(\mathbf{u}_{i}-\boldsymbol{\mu}_{k}^{(r+1)}\right)^{\top} \mid \mathbf{Y}=\mathbf{y}, \boldsymbol{\psi}^{(r)}\right]}{\sum_{i=1}^{K} \sum_{k=1}^{m} \hat{E}_{r+1}\left[c_{i k} \mid \mathbf{Y}=\mathbf{y}, \boldsymbol{\psi}^{(r)}\right]} \\
\boldsymbol{\mu}_{k}^{(r+1)}= & \frac{\sum_{i=1}^{K} \hat{E}_{r+1}\left[c_{i k} \mathbf{u}_{i} \mid \mathbf{Y}=\mathbf{y}, \boldsymbol{\psi}^{(r)}\right]}{\sum_{i=1}^{K} \hat{E}_{r+1}\left[c_{i k} \mid \mathbf{Y}=\mathbf{y}, \boldsymbol{\psi}^{(r)}\right]} \\
\boldsymbol{\Sigma}_{e}^{(r+1)} & \frac{1}{N} \sum_{i=1}^{K} \sum_{j=1}^{n_{i}} \hat{E}_{r+1}\left[\mathbf{e}_{i j}\left(\boldsymbol{\beta}^{(r)}\right) \mathbf{e}_{i j}\left(\boldsymbol{\beta}^{(r)}\right)^{\top} \mid \mathbf{Y}=\mathbf{y}, \boldsymbol{\psi}^{(r)}\right] \\
\boldsymbol{\beta}^{(r+1)}= & \left(\sum_{i=1}^{K} \sum_{j=1}^{n_{i}} \mathbf{x}_{i j}^{\top}\left\{\boldsymbol{\Sigma}_{e}^{(r+1)}\right\}^{-1} \mathbf{x}_{i j}\right)^{-1}\left(\sum_{i=1}^{K} \sum_{j=1}^{n_{i}} \mathbf{x}_{i j}^{\top}\left\{\boldsymbol{\Sigma}_{e}^{(r+1)}\right\}^{-1} \hat{E}_{r+1}\left[\left(\mathbf{y}_{i j}^{*}-\mathbf{z}_{i j} \mathbf{u}_{i}\right) \mid \mathbf{Y}=\mathbf{y}, \boldsymbol{\psi}^{(r)}\right]\right)
\end{aligned}
$$

\subsubsection{Gibbs sampler}

Given $c_{i k}=1$ and $\mathbf{Y}_{i}=\mathbf{y}_{i}, \mathbf{Y}_{i}^{*}$ is distributed according to a truncated multivariate normal mixture. Given $c_{i k}=1$ and $\mathbf{Y}_{i}^{*}=\mathbf{y}_{i}^{*}, \mathbf{u}_{i}$ is distributed according to a multivariate 
normal.

a) Initialization: For each cluster $i$, select any $\mathbf{c}_{i}^{(0)}, \mathbf{u}_{i}^{(0)}$ and $\mathbf{y}_{i}^{*(0)}$ compatible with the observed data $\mathbf{y}_{i}$

b) Iteration $r$ : for each cluster $i$

1. Sampling of the latent responses: Use a Gibbs sampler for truncated normal mixtures to draw $\mathbf{c}_{i}^{(r)}$ and $\mathbf{y}_{i}^{*(r)}$ based on $\mathbf{c}_{i}^{(r-1)}, \mathbf{y}_{i}^{*(r-1)}$ and the current parameter values $\boldsymbol{\psi}^{(r-1)}$

2. Sampling random effects: Select $\mathbf{u}_{i}^{(r)}$ based on the conditional distribution of the random effects given $\mathbf{C}_{i}=\mathbf{c}_{i}^{(r)}, \mathbf{Y}_{i}^{*}=\mathbf{y}_{i}^{*(r)}$ and the current parameter values $\boldsymbol{\psi}^{(r-1)}$

\section{Sampling from a truncated homoschedastic normal mixture}

A generic Gibbs sampler is constructed as follows. Let $\mathbf{Y}^{*}=\left[\begin{array}{c}\mathbf{Y}_{1}^{*} \\ \mathbf{Y}_{2}^{*}\end{array}\right]$ denote a random vector, where $\mathbf{Y}_{1}^{*}$ and $\mathbf{Y}_{2}^{*}$ are respectively $p \times 1$ and $q \times 1$ random vectors. For $m \geq 1$, let $\mathbf{C}$ denote a $0-1 m \times 1$ random vector according to a multinomial distribution with parameters $(1, \mathbf{p})$ for some probability vector $\mathbf{p}$. Given $C_{k}=1, \mathbf{Y}^{*}$ has a multivariate normal distribution with parameters $\boldsymbol{\mu}_{k}$ and $\mathbf{D}$. Finally define $\mathbf{Y}=\left[\begin{array}{c}\mathbf{Y}_{1} \\ \mathbf{Y}_{2}\end{array}\right]$, where $\mathbf{Y}_{1}=\mathbf{Y}_{1}^{*}$ and $Y_{2 i}=I\left\{Y_{2 i}^{*}>0\right\}$ for $i=1, \ldots, q$.

$$
\operatorname{Pr}\left(\mathbf{C}^{(r+1)}=\mathbf{c}^{(r+1)}\right)=\operatorname{Pr}\left(\mathbf{C}=\mathbf{c}^{(r+1)} \mid \mathbf{Y}=\mathbf{y}, \mathbf{Y}_{2}^{*}=\mathbf{y}_{2}^{*(r+1,1)}\right)
$$


where $\mathbf{y}_{2}^{*(r+1,1)}=\mathbf{y}_{2}^{*(r)}$.

For $i=1, \ldots, q$

$$
\operatorname{Pr}\left(Y_{2 i}^{*(r+1)}=y_{2 i}^{*(r+1)}\right)=\operatorname{Pr}\left(Y_{2 i}^{*}=y_{2 i}^{*(r+1)} \mid \mathbf{Y}=\mathbf{y}, \mathbf{Y}_{2(i)}^{*}=\mathbf{y}_{2(i)}^{*(r+1, i+1)} \text { and } \mathbf{C}=\mathbf{c}^{(r+1)}\right)
$$

where $\mathbf{y}_{2(i)}^{*(r+1, i+1)}=\mathbf{y}_{2(i)}^{*(r+1,(i-1)+1)}$.

At the $r$-th iteration, the couple $\left(\mathbf{C}^{(r)}, \mathbf{Y}_{2}^{*(r)}\right)$ is generated. When $r$ is large, $\left(\mathbf{C}^{(r)}, \mathbf{Y}_{2}^{*(r)}\right)$ converges to the desired conditional distribution.

We next provide more detailed expressions for the conditional probabilities. Consider $\mathbf{y}$ and $\mathbf{y}_{2}^{*}$ such that $\{\mathbf{Y}=\mathbf{y}\} \cap\left\{\mathbf{Y}_{2}^{*}=\mathbf{y}_{2}^{*}\right\} \neq \varnothing$, and let $\mathbf{y}^{*}=\left[\begin{array}{c}\mathbf{y}_{1} \\ \mathbf{y}_{2}^{*}\end{array}\right]$.

$\operatorname{Pr}\left(\mathbf{C}=\mathbf{c} \mid \mathbf{Y}=\mathbf{y}, \mathbf{Y}_{2}^{*}=\mathbf{y}_{2}^{*}\right)=\frac{\operatorname{Pr}\left(\mathbf{C}=\mathbf{c} \text { and } \mathbf{Y}^{*}=\mathbf{y}^{*}\right)}{\operatorname{Pr}\left(\mathbf{Y}^{*}=\mathbf{y}^{*}\right)}=\frac{\sum_{k=1}^{m} c_{k} p_{k} \operatorname{Pr}\left(\mathbf{Y}^{*}=\mathbf{y}^{*} \mid C_{k}=1\right)}{\sum_{k=1}^{m} p_{k} \operatorname{Pr}\left(\mathbf{Y}^{*}=\mathbf{y}^{*} \mid C_{k}=1\right)}$

where $\operatorname{Pr}\left(\mathbf{Y}^{*}=\mathbf{y}^{*} \mid C_{k}=1\right)$ is the multivariate normal density with mean $\boldsymbol{\mu}_{k}$ and covariance matrix $\boldsymbol{\Sigma}_{k}$.

For $i=1, \ldots, q$, let $\mu_{2 i}\left(\mathbf{y}_{1}^{*}, \mathbf{y}_{2(i)}^{*}, \mathbf{c}\right)$ and $\sigma_{2 i}\left(\mathbf{y}_{1}^{*}, \mathbf{y}_{2(i)}^{*}, \mathbf{c}\right)$ respectively denote the conditional mean and variance of $Y_{2 i}^{*}$ given $\mathbf{Y}_{1}^{*}=\mathbf{y}_{1}^{*}, \quad \mathbf{Y}_{2(i)}^{*}=\mathbf{y}_{2(i)}^{*}$, and $\mathbf{C}=\mathbf{c}$.

$$
\begin{aligned}
& \operatorname{Pr}\left(Y_{2 i}^{*}=y_{2 i}^{*} \mid \mathbf{Y}=\mathbf{y}, \mathbf{Y}_{2(i)}^{*}=\mathbf{y}_{2(i)}^{*} \text { and } \mathbf{C}=\mathbf{c}\right)= \\
& I\left\{y_{2 i}^{*}>0\right\} \frac{\Phi\left(\frac{\mathbf{y}_{2 i}^{*}-\mu_{2 i}}{\sigma_{2 i}}\right)-\Phi\left(-\frac{\mu_{2 i}}{\sigma_{2 i}}\right)}{1-\Phi\left(-\frac{\mu_{2 i}}{\sigma_{2 i}}\right)}+I\left\{y_{2 i}^{*} \leq 0\right\} \frac{\Phi\left(\frac{\mathbf{y}_{2 i}^{*}-\mu_{2 i}}{\sigma_{2 i}}\right)}{\Phi\left(-\frac{\mu_{2 i}}{\sigma_{2 i}}\right)}
\end{aligned}
$$




\section{Sampling of random effects}

Conditional on $c_{i k}=1,\left(\mathbf{u}_{i}, \mathbf{Y}_{i}^{*}\right)$ has a multivariate normal distribution as follows

$$
\left(\begin{array}{c}
\mathbf{Y}_{i}^{*} \\
\mathbf{u}_{i}
\end{array}\right) \sim N\left(\left(\begin{array}{c}
\mathbf{X}_{i} \boldsymbol{\beta}+\mathbf{Z}_{i} \boldsymbol{\mu}_{k} \\
\boldsymbol{\mu}_{k}
\end{array}\right),\left(\begin{array}{rr}
\mathbf{Z}_{i} \mathbf{D} \mathbf{Z}_{i}^{\top}+\boldsymbol{\Sigma}_{E_{i}} & \mathbf{Z}_{i} \mathbf{D} \\
\mathbf{D Z}_{i}^{\top} & \mathbf{D}
\end{array}\right)\right)
$$

Therefore

$$
\mathbf{u}_{i} \mid\left\{\mathbf{Y}_{i}^{*}=\mathbf{y}_{i}^{*}, \text { and } c_{i k}=1\right\} \sim N\left(\boldsymbol{\mu}_{k}+\boldsymbol{\Sigma}_{B_{i}}\left(\mathbf{y}_{i}^{*}-\mathbf{X}_{i} \boldsymbol{\beta}-\mathbf{Z}_{i} \boldsymbol{\mu}_{k}\right), \mathbf{D}-\boldsymbol{\Sigma}_{B_{i}} \mathbf{Z}_{i} \mathbf{D}\right)
$$

where

$$
\boldsymbol{\Sigma}_{E_{i}}=\boldsymbol{\Sigma}_{e} \otimes \boldsymbol{I}_{n_{i}}=\left[\begin{array}{ccc}
\boldsymbol{\Sigma}_{e} & \ldots & 0 \\
\vdots & \ddots & \vdots \\
& & \\
0 & \ldots & \boldsymbol{\Sigma}_{e}
\end{array}\right]
$$

and

$$
\boldsymbol{\Sigma}_{B_{i}}=\boldsymbol{\Sigma} \mathbf{Z}_{i}^{\top}\left(\mathbf{Z}_{i} \boldsymbol{\Sigma} \mathbf{Z}_{i}^{\top}+\boldsymbol{\Sigma}_{E_{i}}\right)^{-1}
$$




\section{Chapter 6}

\section{Simulations}

In this chapter, we analyse the performance of the proposed SAECM algorithm through simulations. Different scenarios were considered where data were generated according to the following correlated probit model with latent responses:

$$
\begin{aligned}
& y_{i j 1}^{*}=\beta_{01}+\beta_{11} x_{i}+u_{i 1}+\epsilon_{i j 1} \\
& y_{i j 2}^{*}=\beta_{02}+\beta_{12} x_{i}+u_{i 2}+\epsilon_{i j 2} \\
& y_{i j 1}=y_{i j 1}^{*} \\
& y_{i j 2}=I\left(\left\{y_{i j 2}^{*}>0\right\}\right)
\end{aligned}
$$

where $\epsilon_{i j 1}, \epsilon_{i j 2}$ are iid according to $N(0,1)$. As mentioned before, only $y_{i j 1}=y_{i j 1}^{*}$ (continuous response) and $y_{i j 2}$ (binary response) were observed. For all the scenarios we considered $K=100$ clusters, and the explanatory variable was set as follows:

$$
x_{i}=\left\{\begin{aligned}
0 & \text { if } i=1, \ldots, K / 2 \\
-1 & \text { if } i=K / 2+1, \ldots, K / 2+K / 4 \\
1 & \text { if } i=K / 2+K / 4+1, \ldots, K
\end{aligned}\right.
$$


As mentionned before, the parameters of the model are

- $\boldsymbol{\beta}=\left[\begin{array}{llll}\beta_{01} & \beta_{11} & \beta_{02} & \beta_{12}\end{array}\right]^{\top}$ the vector of regression coefficients

$-\boldsymbol{\Sigma}_{e}=\left[\begin{array}{cc}\sigma_{e, 11} & \sigma_{e, 12} \\ \sigma_{e, 21} & \sigma_{e, 22}\end{array}\right]$ the covariance of the random errors

- D, the common covariance matrix of mixture components of random effects

- the probability $p_{g}$ and the mean $\boldsymbol{\mu}_{g}=\left[\mu_{g 1} \mu_{g 2}\right]^{\top}$ of each mixture component of the random effects

As for $\boldsymbol{\Sigma}=\left[\begin{array}{cc}\sigma_{11} & \sigma_{12} \\ \sigma_{21} & \sigma_{22}\end{array}\right]$, the random effects covariance matrix, it is obtained from the mixture components based on Equation 4.23. The model is not identifiable without assuming a given value for $\sigma_{e, 22}$, the error variance for the binary response, e.g. $\sigma_{e, 22}=$ 1 as sugested by Gueorgieva and Agresti [20]. This is equivalent to a reparametrization of the model, where a subset of the parameters are substituted as follows:

$$
\begin{aligned}
\beta_{02} & \rightarrow \frac{\beta_{02}}{\sqrt{\sigma_{e, 22}}} \\
\beta_{12} & \rightarrow \frac{\beta_{12}}{\sqrt{\sigma_{e, 22}}} \\
\sigma_{22} & \rightarrow \frac{\sigma_{22}}{\sigma_{e, 22}} \\
\sigma_{12} & \rightarrow \frac{\sigma_{12}}{\sqrt{\sigma_{e, 22}}} \\
\sigma_{e, 12} & \rightarrow \frac{\sigma_{e, 12}}{\sqrt{\sigma_{e, 22}}} \\
\mu_{g 2} & \rightarrow \frac{\mu_{g 2}}{\sqrt{\sigma_{e, 22}}}
\end{aligned}
$$

Although no rigorous proof has been given of this fact, in keeping with [20], we assume that the new parametrization makes the model identifiable. In fact, a full discussion of the model identifiability extends well beyond the scope of this work. 
For each setting 100 independent samples were generated and used to produce ML estimates. Altough it was desirable to simulate a larger sample size (e.g. 1000 independent samples for each setting), we were limited by the slow convergence of the algorithm $^{1}$, a common shortcoming of EM algorithms.

In the simulations, a value of $\alpha=0.75$ was used as suggested by Delyon et al. [22], and convergence was detected when the estimated observed information matrix becomes positive definite ${ }^{2}$ for a sufficient number of consecutive iterations (set to 200 in the simulations) of the SAECM algorithm. In the literature different stopping criteria were proposed, e.g. based on running independent simulations in parallel with the same initial values of parameters, and detecting stationarity by comparing the different estimates [21]. However that choice would have resulted in even longer simulations.

\subsection{Random effects with a normal distribution}

We consider two scenarios with cluster sizes of $n=2,4$ and random effects according to $N\left(\mathbf{0}, \mathbf{I}_{2}\right)$, where $\mathbf{I}_{2}$ is the $2 \times 2$ identity matrix. In this case the SAECM algorithm is identical to that of Gueorguieva and Agresti [20]. Datasets were generated using the values given in Table 6.1. For a cluster size of $n=2$, the average number of iterations per dataset was 5853.84 with a standard deviation of 4377.04 . The number of iterations is significantly reduced when the cluster size increases. Indeed when $n=$ 4 , the average number of iterations per dataset was 3482.46 with a standard deviation of 2192.54 . This is nearly a $40 \%$ reduction in the average number of iterations. The average number of iterations may seem large, however it compares well with the number of iterations (10000 iterations for about 100 clusters, and cluster sizes around 10) reported by R. Gueorguieva and A. Agresti [20] for their SAECM algorithm.

\footnotetext{
${ }^{1}$ On a single processor machine, individual runs required up to 12 hours to complete

${ }^{2}$ Based on its eigenvalues
} 
It is also important to keep in mind that stochastic approximation generates one conditional sample of the complete data for each cluster in the $\mathrm{E}$ step. Therefore the E step requires much less computation than with the traditional EM or ECM algorithms where hundreds of conditional samples may be generated for each cluster. The results are presented in Table 6.2. For $\hat{\beta}_{01}$, the empirical mean, standard deviation Table 6.1: Parameters values when the random effects have a normal distribution.

\begin{tabular}{lcccccccccc}
\hline Parameters & $\beta_{01}$ & $\beta_{02}$ & $\beta_{11}$ & $\beta_{12}$ & $\sigma_{11}$ & $\sigma_{22}$ & $\sigma_{12}$ & $\sigma_{e, 11}$ & $\sigma_{e, 22}$ & $\sigma_{e, 12}$ \\
\hline Values & 1 & 1 & 1 & 1 & 1 & 1 & 0 & 1 & 1 & 0 \\
\hline
\end{tabular}

and MSE are computed as follows,

$$
\begin{aligned}
\operatorname{mean}\left(\hat{\beta}_{01}\right) & =\frac{1}{S} \sum_{s=1}^{S} \hat{\beta}_{01, s} \\
\operatorname{std}\left(\hat{\beta}_{01}\right) & =\frac{1}{S-1} \sqrt{\sum_{s=1}^{S}\left[\hat{\beta}_{01, s}-\operatorname{mean}\left(\hat{\beta}_{01}\right)\right]^{2}} \\
\operatorname{MSE}\left(\hat{\beta}_{01}\right) & =\frac{1}{S} \sum_{s=1}^{S}\left(\hat{\beta}_{01, s}-\beta_{01}\right)^{2}
\end{aligned}
$$

where $\hat{\beta}_{01, s}$ is the MLE for the $s$-th sample and $S=100$ is the number of samples. The empirical results are computed in the similar manner for the other parameters and expressions. Actual values of parameters are shown in parenthesis in Table 6.2. In the table, the parameters affecting the binary response have been rescaled by the variance of the random error for the binary response. As mentionned before, this step is necessary to obtain an identifiable model.

The results show a small MLE bias for fixed and random effects parameters. Also the standard errors are larger for parameters affecting the binary responses, than for their counterparts in the continuous responses. For example the standard error and MSE of $\frac{\hat{\beta}_{02}}{\sqrt{\hat{\sigma}_{e, 22}}}$ are both larger than these of $\hat{\beta}_{01}$. This is expected because the fraction of 
missing information is larger for the binary response than for the continuous response. For all the parameters, the MSE is reduced when the cluster size is increased from $n=2$ to $n=4$.

Table 6.2: MLE statistics for fixed effects and random effects parameters, in a correctly specified model with normal random effects, $K=100$ and $n=2,4$.

\begin{tabular}{|c|c|c|c|c|c|c|c|c|c|}
\hline & $\begin{array}{l}\hat{\beta}_{01} \\
\text { (1) }\end{array}$ & $\begin{array}{c}\frac{\hat{\beta}_{02}}{\sqrt{\hat{\sigma}_{e, 22}}} \\
(1)\end{array}$ & $\begin{array}{l}\hat{\beta}_{11} \\
(1)\end{array}$ & $\begin{array}{c}\frac{\hat{\beta}_{12}}{\sqrt{\hat{\sigma}_{e, 22}}} \\
(1)\end{array}$ & $\begin{array}{l}\hat{\sigma}_{11} \\
(1)\end{array}$ & $\begin{array}{c}\frac{\hat{\sigma}_{22}}{\hat{\sigma}_{e, 22}} \\
(1)\end{array}$ & $\begin{array}{c}\frac{\hat{\sigma}_{12}}{\sqrt{\hat{\sigma}_{e, 22}}} \\
(0)\end{array}$ & $\begin{array}{r}\hat{\sigma}_{e, 11} \\
(1)\end{array}$ & $\begin{array}{c}\frac{\hat{\sigma}_{e, 12}}{\sqrt{\hat{\sigma}_{e, 22}}} \\
(0)\end{array}$ \\
\hline \multicolumn{10}{|c|}{$\mathrm{n}=$} \\
\hline Mean & 1.0114 & 1.0187 & 1.0008 & 1.0162 & 0.9619 & 1.0471 & -0.0062 & .9757 & 0.0277 \\
\hline Std & 7 & 0.1780 & 0.1578 & 0.2427 & 0.1938 & 0.4821 & 0.2009 & 0.1429 & 0.1805 \\
\hline Bias & 114 & 0.0187 & 0.0008 & 0.0162 & -0.0412 & 0.0059 & -0.0062 & -0.0243 & 0.0277 \\
\hline & 0.1266 & 0.1781 & 0.1570 & 0.2420 & 0.1966 & 0.4820 & 0.2000 & 0.1442 & 0.1817 \\
\hline \multicolumn{10}{|c|}{$\mathrm{n}=4$} \\
\hline Mean & 1.0085 & 0.9948 & 0.9763 & 0.9742 & 0.9812 & 0.9716 & 0.0089 & 23 & 0.0063 \\
\hline Std & 0.0997 & 0.1466 & 0.1460 & 0.1989 & 0.1755 & 0.3285 & 0.1579 & 0.0869 & 0.1045 \\
\hline Bias & 0.0085 & -0.0052 & -0.0237 & -0.0258 & -0.0357 & -0.0265 & 0.0089 & -0.0077 & 0.0063 \\
\hline$\sqrt{\mathrm{MSE}}$ & 0.0996 & 0.1459 & 0.1472 & 0.1996 & 0.1756 & 0.3281 & 0.1573 & 0.0868 & 0.1041 \\
\hline
\end{tabular}

\subsection{Random effects following a normal mixture}

In this section, the random effects are gerenated according to an homoschedastic normal mixture with two components $\pi N\left(\boldsymbol{\mu}_{1}, \mathbf{D}\right)+(1-\pi) N\left(\left(\boldsymbol{\mu}_{2}, \mathbf{D}\right)\right.$ where

$$
\pi=\frac{4}{5}, \quad \boldsymbol{\mu}_{1}=\left[-\frac{5}{4}-\frac{5}{4}\right]^{\top}, \quad \boldsymbol{\mu}_{2}=\left[\begin{array}{ll}
5 & 5
\end{array}\right]^{\top}, \quad \mathbf{D}=\left[\begin{array}{ll}
d_{11} & d_{12} \\
d_{21} & d_{22}
\end{array}\right]=\left[\begin{array}{ll}
1 & 0 \\
0 & 1
\end{array}\right]
$$

Two hundred independent data samples are generated, among which the first half is fitted assuming normal random effects, while the second half is fitted assuming a 
normal mixture with two components. The other parameters are set as in Table 6.1.

\subsubsection{Misspecified model}

In the misspecified scenario, the parameters are estimated asuming normal random effects. For a cluster size of $n=2$, the algorithm converges in 8073.93 iterations on average, with a standard deviation of 4548.31 . The convergence is faster with a larger cluster size of $n=4$, where the average number of iterations per dataset is 6690.98 with a standard deviation of 3320.76. The results are shown in Table 6.3. They show a significant MLE bias and MSE for fixed and random effects parameters, except for $\hat{\beta}_{01}$. For all fixed effect parameters except $\beta_{01}$, the relative bias is between $25 \%$ and $40 \%$. For random effect parameters (i.e. $\sigma_{11}, \sigma_{22}$ and $\sigma_{12}$ ), the relative bias exceeds 40\%. Therefore the misspecification has a significant impact. However it has little impact on the parameters of the random error.

When the cluster size is increased, two different trends are observed for the MLEs of fixed effects parameters on one hand, and the MLEs of variance components (random effects and random errors) on the other hand. For fixed effects, the MSEs become larger. The opposite trend is observed for variance components, i.e. their MSEs become smaller. The impact of a larger cluster size on the MLE bias is more complex to analyse. It is not possible to separate fixed effects and random effects parameters based on the direction of change to their MLE biases.

\subsubsection{Correct model}

One hundred independent datasets are next generated and fitted using the correct random effects model, i.e. a normal mixture with two components. In the interest of time, the maximum number of iterations was fixed at 50000 and it was assumed that the SAECM did not converge when this number was reached. According to 
Table 6.3: MLE statistics of fixed effets and random effects, in a misspecified model with random effects following a two-components normal mixture but fitted assuming normal random effects, with $K=100$ and $n=2,4$.

\begin{tabular}{|c|c|c|c|c|c|c|c|c|c|}
\hline & $\begin{array}{l}\hat{\beta}_{01} \\
(1) \\
\end{array}$ & $\begin{array}{c}\frac{\hat{\beta}_{02}}{\sqrt{\hat{\sigma}_{e, 22}}} \\
(1)\end{array}$ & $\begin{array}{l}\hat{\beta}_{11} \\
(1)\end{array}$ & $\begin{array}{c}\frac{\hat{\beta}_{12}}{\sqrt{\hat{\sigma}_{e, 22}}} \\
(1)\end{array}$ & $\begin{array}{c}\hat{\sigma}_{11} \\
(29 / 4) \\
\end{array}$ & $\begin{array}{c}\hat{\sigma}_{22} \\
\hat{\sigma}_{e, 22} \\
(29 / 4)\end{array}$ & $\begin{array}{c}\frac{\hat{\sigma}_{12}}{\sqrt{\hat{\sigma}_{e, 22}}} \\
(25 / 4)\end{array}$ & $\begin{array}{c}\hat{\boldsymbol{\sigma}}_{e, 11} \\
(1) \\
\end{array}$ & $\begin{array}{c}\frac{\hat{\sigma}_{e, 12}}{\sqrt{\hat{\sigma}_{e, 22}}} \\
(0)\end{array}$ \\
\hline \multicolumn{10}{|c|}{$\mathrm{n}=2$} \\
\hline Mean & 1.0022 & 0.6130 & 0.7232 & 0.7326 & 4.8879 & 2.1615 & 1.9647 & 0.9983 & 0.0027 \\
\hline Std & 0.2572 & 0.4076 & 0.3177 & 0.3703 & 3.0987 & 1.5307 & 1.7151 & 0.1532 & 0.1654 \\
\hline Bias & 0.0022 & -0.3870 & -0.2768 & -0.2674 & -2.3621 & -5.0885 & -4.2853 & -0.0017 & 0.0027 \\
\hline$\sqrt{\mathrm{MS}}$ & 0.2559 & 0.5606 & 0.4201 & 0.4553 & 3.8840 & 5.3115 & 4.6125 & 0.1524 & 0.1646 \\
\hline \multicolumn{10}{|c|}{$\mathrm{n}=4$} \\
\hline Mean & 1.0030 & 0.3802 & 0.5057 & 0.4908 & 7.0085 & 2.8234 & 3.2292 & 1.0061 & -0.0061 \\
\hline Std & 0.2709 & 0.2030 & 0.2054 & 0.1982 & 1.0053 & 1.0096 & 0.8986 & 0.0818 & 0.0968 \\
\hline Bias & 0.0030 & -0.6198 & -0.4943 & -0.5092 & -0.2415 & -4.4266 & -3.0208 & 0.0061 & -0.0061 \\
\hline$\sqrt{\mathrm{MSE}}$ & 0.2695 & 0.6518 & 0.5349 & 0.5461 & 1.0290 & 4.5391 & 3.1503 & 0.0817 & 0.0965 \\
\hline
\end{tabular}

this convention, the SAECM did not converge for seven datasets. Results for those datasets are ignored. For a clsuter size of $n=2$, the SAECM algorithm converges in 19589.69 iterations on average ${ }^{3}$, with a standard deviation of 11066.36 . For a cluster size of $n=4$, the average number of iterations is slightly reduced to 14146.03 with a standard deviation of 10968.31. The MLE statistics are presented in Table 6.4 and Table 6.5. Overall, the SAECM algorithm produces MLEs with small biases as expected, both for fixed effects parameters, and random effects parameters. The standard errors and MSEs are significantly smaller (by a factor of two or three) than in the misspecified case except for $\beta_{01}$, where they are actually larger. Mixture components are also estimated with small biases.

For most parameters and expressions, the MSE is reduced when the cluster size increases. The only exceptions are the intercept parameters $\left(\beta_{01}\right.$ and $\left.\beta_{02} / \sqrt{\sigma_{e, 22}}\right)$.

\footnotetext{
${ }^{3}$ When the SAECM algorithm converges
} 
Table 6.4: MLE statistics of fixed effets and random effects, in a correctly specified model with random effects following a two-components normal mixture, with $K=100$ and $n=2,4$.

\begin{tabular}{lccccccccc}
\hline & $\hat{\beta}_{01}$ & $\begin{array}{c}\hat{\beta}_{02} \\
\sqrt{\hat{\sigma}_{e, 22}} \\
(1)\end{array}$ & $\begin{array}{c}\hat{\beta}_{11} \\
(1)\end{array}$ & $\begin{array}{c}\frac{\hat{\beta}_{12}}{\sqrt{\hat{\sigma}_{e, 22}}} \\
(1)\end{array}$ & $\begin{array}{c}\hat{\sigma}_{11} \\
(29 / 4)\end{array}$ & $\begin{array}{c}\frac{\hat{\sigma}_{22}}{\hat{\sigma}_{e, 22}} \\
(29 / 4)\end{array}$ & $\begin{array}{c}\frac{\hat{\sigma}_{12}}{\sqrt{\hat{\sigma}_{e, 22}}} \\
(25 / 4)\end{array}$ & $\begin{array}{c}\hat{\sigma}_{e, 11} \\
(1)\end{array}$ & $\begin{array}{c}\frac{\hat{\sigma}_{e, 12}}{\sqrt{\hat{\sigma}_{e, 22}}} \\
(0)\end{array}$ \\
\hline Mean & 1.0065 & 1.0025 & 0.9889 & 1.0456 & 6.9882 & 7.4978 & 6.1590 & 1.0114 & -0.0029 \\
Std & 0.2733 & 0.2827 & 0.1836 & 0.3191 & 1.4212 & 2.3881 & 1.5103 & 0.1457 & 0.1705 \\
Bias & 0.0065 & 0.0025 & -0.0111 & 0.0456 & -0.2618 & 0.2478 & -0.0910 & 0.0114 & -0.0029 \\
$\sqrt{\text { MSE }}$ & 0.2718 & 0.2811 & 0.1829 & 0.3205 & 1.4372 & 2.3874 & 1.5044 & 0.1453 & 0.1696 \\
\hline & & & \multicolumn{6}{c}{$\mathrm{n}=4$} \\
\hline Mean & 1.0350 & 1.0354 & 0.9968 & 1.0090 & 7.3132 & 7.2837 & 6.3397 & 1.0131 & -0.0054 \\
Std & 0.2680 & 0.2985 & 0.1861 & 0.2149 & 1.1450 & 1.6671 & 1.1909 & 0.0818 & 0.1023 \\
Bias & 0.0350 & 0.0354 & -0.0032 & 0.0090 & 0.0632 & 0.0337 & 0.0897 & 0.0131 & -0.0054 \\
$\sqrt{\text { MSE }}$ & 0.2689 & 0.2991 & 0.1852 & 0.2140 & 1.1410 & 1.6591 & 1.1883 & 0.0824 & 0.1019 \\
\hline
\end{tabular}

Table 6.5: MLE statistics for mixture components, in a correctly specified model with random effects following a two-components normal mixture, with $K=100$ and $n=2,4$.

\begin{tabular}{|c|c|c|c|c|c|c|c|c|}
\hline & $\begin{array}{c}\hat{\pi} \\
(4 / 5) \\
\end{array}$ & $\begin{array}{c}\hat{\mu}_{11}+\hat{\beta}_{01} \\
(-1 / 4) \\
\end{array}$ & $\begin{array}{c}\frac{\hat{\mu}_{12}+\hat{\beta}_{02}}{\sqrt{\hat{\sigma}_{e, 22}}} \\
(-1 / 4) \\
\end{array}$ & $\begin{array}{c}\hat{\mu}_{21}+\hat{\beta}_{01} \\
(6) \\
\end{array}$ & $\begin{array}{c}\frac{\hat{\mu}_{22}+\hat{\beta}_{02}}{\sqrt{\hat{\sigma}_{e, 22}}} \\
(6)\end{array}$ & $\begin{array}{l}\hat{d}_{11} \\
(1)\end{array}$ & $\begin{array}{c}\frac{\hat{d}_{22}}{\hat{\sigma}_{e, 22}} \\
(1) \\
\end{array}$ & $\begin{array}{c}\frac{\hat{d}_{21}}{\sqrt{\hat{\sigma}_{e, 22}}} \\
(0)\end{array}$ \\
\hline \multicolumn{9}{|c|}{$\mathrm{n}=2$} \\
\hline Mean & 0.791 & -0.238 & -0.258 & 5.906 & 6.002 & 0.940 & 1.143 & 0.007 \\
\hline Std & 0.073 & 0.161 & 0.188 & 0.766 & 1.010 & 0.227 & 0.520 & 0.199 \\
\hline Bias & -0.009 & 0.012 & -0.008 & -0.094 & 0.002 & -0.060 & 0.143 & 0.007 \\
\hline$\sqrt{\mathrm{MSE}}$ & 0.073 & 0.161 & 0.187 & 0.767 & 1.004 & 0.234 & 0.536 & 0.198 \\
\hline \multicolumn{9}{|c|}{$\mathrm{n}=4$} \\
\hline Mean & 0.794 & -0.254 & -0.248 & 6.012 & 5.976 & 0.954 & 0.961 & 0.022 \\
\hline Std & 0.042 & 0.127 & 0.146 & 0.266 & 0.411 & 0.162 & 0.322 & 0.188 \\
\hline Bias & -0.006 & -0.004 & 0.002 & 0.012 & -0.024 & -0.046 & -0.039 & 0.022 \\
\hline$\sqrt{\mathrm{MSE}}$ & 0.042 & 0.126 & 0.146 & 0.265 & 0.410 & 0.168 & 0.323 & 0.188 \\
\hline
\end{tabular}




\subsection{Discussion}

The results show that misspecification results in significant MLE bias when random effects follow a normal mixture with two widely different components, while the data is fitted assuming they are normal. In this case, the proposed SAECM algorithm provides MLEs with significantly less bias, and with smaller standard errors. However it requires more iterations to converge and each iteration requires more computations. 


\section{Chapter 7}

\section{Conclusion}

According to the results presented in this work, misspecified random effects have a significant impact of the efficiency of MLEs of fixed and random effects parameters, in correlated probit models with continuous and binary responses. It is important to note that this impact is particularly observed in the MLEs of the variance components. Hence these results further emphasize the need to account for random effect misspecification in GLMMs, as highlighted by previous studies considering very large random effects $[15,16]$. The proposed SAECM algorithm computes accurate MLEs, when the random effects follow a normal mixture, according to the heterogeneity model. However like other EM algorithms it converges slowly and even more so due to the underlying mixture model. This is not only due to the linear rate of convergence of EM algorithms in general, but also due to the fact that mixture models often have numerous local extrema of the log-likelihood function, and the question of their

identifiability is not trivial. However these drawbacks are balanced by the numerical stability and monotone convergence of the algorithm, which ensure convergence to a global optimum of the observed log-likelihood under certain conditions [19,22].

In the future, it is desirable to assess the performance of the SAECM algorithm through more extensive simulations based on more complex correlated probit models with a large number of nested random effects. Such models are currently beyond the 
reach of the best Adaptive Gaussian Quadrature methods and are the raison d'être of Monte Carlo EM methods.

Another area of research is that of the estimation of the number of mixture components which we have assumed known in this work.

It is also of interest to investigate faster algorithms for fitting correlated probit models with a mixture distribution of random effects. In the past faster EM algorithms were obtained using the technique of Parameter Extension (PX) proposed by Liu et al. [26]. Unfortunately that method was not successfully applied to mixture models. [26]. An alternative is to use Monte Carlo Newton Raphson algorithms that generally have a quadratic rate of convergence and are theoretically faster than EM algorithms. In that case special attention must be paid to the numerical stability of the resulting algorithms as this has been the major limitation of Newton-Raphson algorithms. Finally the question of model identifiability is fundamental because it conditions our ability to consistently estimate model parameters based on the observed data. Two related questions concern the existence and uniqueness of MLEs in correlated probit models when random effects have a mixture normal distribution. Answers to these questions will inform us regarding the potential divergence of ML algorithms. 


\section{List of References}

[1] C. McCulloch and R. Searle, Generalized, Linear and Mixed Models. New York: John and Wiley and Sons, 2000.

[2] K. Liang and S. Zeger, "Longitudinal data analysis using generalized linear models," Biometrika, vol. 73, pp. 13-22, 1986.

[3] J. Lindsey and P. Lambert, "On the appropriateness of marginal models for repeated measurements in clinical trials," Statistics in medecine, vol. 17, p. 447469, 1998.

[4] Y. Lee and J. Nelder, "Conditional and marginal models: Another view," Statistical Science, vol. 19, pp. 219-228, 2004.

[5] G. Verbeke and E. Lesaffre, "A linear mixed-effects model with heterogeneity in the random-effects population," JASA, vol. 91, pp. 217-221, 1996.

[6] H. White, "Maximum likelihood estimation of misspecified models," International Statistical Review, vol. 50, pp. 1-25, 1982.

[7] A. Agresti, Categorical Data Analysis. Hoboken, New Jersey: John Wiley and Sons, 2002.

[8] J. Neuhaus, W. Hauck, and J. Kalbfleisch, "A comparison of cluster-specific and population-averaged approaches for analyzing correlated binary data," International Statistical Review, vol. 59, pp. 25-35, 1991.

[9] J. Neuhaus, W. Hauck, and J. Kalbfleisch, "The effects of mixture distribution misspecification when fitting mixed-effects logistic models," Biometrika, vol. 79, p. $755762,1992$.

[10] J. Neuhaus, W. Hauck, and J. Kalbfleisch, "Conditions for consistent estimation in mixed-effects models for binary matched-pairs data," Canadian Journal of Statistics, vol. 22, p. 139148, 1994. 
[11] P. Heagerty and B. Kurland, "Misspecified maximum likelihood estimates and generalised linear mixed models," Biometrika, vol. 88, pp. 973-985, 2001.

[12] G. Verbeke and E. Lesaffre, "The effect of misspecifying the random-effects distribution in linear mixed models for longitudinal data," Computational Statistics and Data Analysis, vol. 53, pp. 541-556, 1997.

[13] C. Proust and H. Jacqmin-Gadda, "Estimation of linear mixed models with a mixture of distribution for the random effects," Computer Methods and Programs in Biomedicine, vol. 78, p. 165173, 2005.

[14] A. Agresti, P. Ohman, and B. Caffo, "Examples in which misspecification of a random effects distribution reduces efficiency," Computational Statistics and Data Analysis, vol. 47, pp. 639-653, 2004.

[15] S. Litiere, A. Alonso, and G. Molenberghs, "Type i and type ii error under random-effects misspecification in generalized linear mixed models," Biometrics, vol. 63, pp. 1038-1044, 2006.

[16] S. Litiere, A. Alonso, and G. Molenberghs, "Generalized linear mixed models with non-gaussian random effects." Technical report 0657 available at http://www.stat.ucl.ac.be/ISpub/tr/2006/TR0657.pdf, 2006.

[17] M. Aitkin, "A general maximum likelihood analysis of variance components in generalized linear models," Biometrics, vol. 55, p. 117128, 1999.

[18] D. Follman and D. Lambert, "Identifiability of finite mixtures of logistic regression models," J. Statist. Plann. Inference, vol. 27, p. 375381, 1991.

[19] A. Dempster, N. Laird, and D. Rubin, "Maximum likelihood from incomplete data via the em algorithm," Journal of the Royal Statistical Society. Series B, vol. 39, pp. 1-38, 1977.

[20] R. Gueorguieva and A. Agresti, "A correlated probit model for joint modeling of clustered binary binary and continuous responses," Journal of the American Statistical Association, vol. 96, pp. 1102-1112, 2001.

[21] J. Chan and A. Kuk, "Maximum likelihood estimation for probit-linear mixed models with correlated random effects," Biometrics, vol. 53, pp. 86-97, 1997.

[22] B. Delyon, M. Lavielle, and E. Moulines, "On a stochastic approximation version of the em algorithmn," The Annals of Statistics, vol. 27, pp. 94-128, 1999. 
[23] T. Louis, "Finding the observed information matrix when using the em algorithm," Journal of the Royal Statistical Society B, vol. 44, pp. 226-233, 1982.

[24] X. Meng and D. Rubin, "Maximum likelihood estimation via the ecm algorithm. a general framework," Biometrika, vol. 80, pp. 267-278, 1993.

[25] T. Kollo, Advanced Multivariate Statistics with Matrices. New York: Springer, 2005.

[26] C. Liu, D. Rubin, and Y. Wu, "Expansion to accelerate em: The px-em algorithm," Biometrika, vol. 85, pp. 755-770, 1998. 


\section{Appendix A}

\section{Matrix theory}

\section{A.1 Partitioned and patterned matrices}

Definition 2 (partitioned matrix) A $p \times q$ matrix $\mathbf{A}$ is called a partitioned matrix (or block matrix) if it consists of uv submatrices $\mathbf{A}_{i j}$ of size $p_{i} \times q_{j}$ so that

$$
\mathbf{A}=\left[\begin{array}{llll}
\mathbf{A}_{11} & \mathbf{A}_{12} & \ldots & \mathbf{A}_{1 v} \\
\vdots & \vdots & \ddots & \vdots \\
& & & \\
\mathbf{A}_{u 1} & \mathbf{A}_{u 2} & \ldots & \mathbf{A}_{u v}
\end{array}\right], \sum_{i=1}^{u} p_{i}=p ; \quad \sum_{j=1}^{v} q_{j}=q
$$

The partitioned matrix is denoted by $\mathbf{A}=\left[\mathbf{A}_{i j}\right]_{1 \leq i \leq u, 1 \leq j \leq v}$. For $(i, j) \in\{1, \ldots, u\} \times$ $\{1, \ldots, v\}$ and $(k, l) \in\left\{1, \ldots, p_{i}\right\} \times\left\{1, \ldots, q_{j}\right\}$ let $a_{(i, j)(k, l)}$ denote the entry $(k, l)$ in the block $\mathbf{A}_{i j}$.

Definition 3 (commutation matrix) The commutation matrix $\mathbf{K}_{p, q}$ is the $(p q) \times$ ( $p q$ ) matrix consisting of $q \times p$ blocks such that

$$
\left(\mathbf{K}_{p, q}\right)_{(i, j)(g, h)}= \begin{cases}1 & \text { if } g=j \text { and } h=i \\ 0 & \text { else }\end{cases}
$$




\section{A.2 Vectorized matrices}

For $\mathbf{X} \in \mathbb{R}^{p \times q}$, let $\mathbf{X}_{i, \text { : }}$ denote the $i$-th row, and $\mathbf{X}_{i, i}$ the $i$-th column of $\mathbf{X}$. Then the vectorization operator is defined as follows

$$
\operatorname{vec}(\mathbf{X})=\left[\begin{array}{lll}
\mathbf{X}_{1,:} & \ldots & \mathbf{X}_{p,}
\end{array}\right]^{\top}
$$

\section{A.3 Differentiation}

Define the partial differential operator

$$
\frac{\partial}{\partial \mathbf{X}}=\left[\frac{\partial}{\partial X_{i j}}\right]_{1 \leq i \leq p, 1 \leq j \leq q}
$$

It maps a matrix $\mathbf{Y}$ to $\frac{\partial}{\partial \mathbf{X}} \otimes \mathbf{Y}$.

Define the matrix derivative $\frac{d \mathbf{Y}}{d \mathbf{X}}$ as follows

$$
\frac{d \mathbf{Y}}{d \mathbf{X}}=\frac{\partial}{\partial \operatorname{vec}(\mathbf{X})} \operatorname{vec}(\mathbf{Y})^{\top}=\frac{\partial}{\partial \operatorname{vec}(\mathbf{X})} \otimes \operatorname{vec}(\mathbf{Y})^{\top}
$$

There are alternative definitions of matrix derivatives. However the above form preserves more properties of traditional derivatives, e.g. the chain rule.

From [25].

Define

$$
\frac{\partial^{2} \mathbf{Y}}{\partial \mathbf{X} \partial \mathbf{X}^{\top}}=\frac{\partial}{\partial \mathbf{X}}\left\{\frac{\partial \mathbf{Y}}{\partial \mathbf{X}}\right\}^{\top}
$$




\begin{tabular}{|c|c|}
\hline $\mathbf{Y}=f(\mathbf{X}), \mathbf{X} \in \mathbb{R}^{p \times q}$ & $\frac{d \mathbf{Y}}{d \mathbf{X}}$ \\
\hline $\mathbf{x}$ & $\mathbf{I}_{p q}$ \\
\hline $\mathbf{X}^{\top}$ & $\mathbf{K}_{q, p}$ \\
\hline $\mathrm{X}^{-1}$ & $-\mathbf{X}^{-1} \otimes\left(\mathbf{X}^{\top}\right)^{-1}$ \\
\hline$|\mathbf{X}|, \mathbf{X} \in \mathbb{R}^{p \times p}$ & $|\mathbf{X}| \operatorname{vec}\left(\left\{\mathbf{X}^{-1}\right\}^{\top}\right)$ \\
\hline $\mathbf{A}^{\top} \operatorname{vec}(\mathbf{X})$ & $\mathbf{A}$ \\
\hline $\operatorname{tr}\left(\mathbf{A}^{\top} \mathbf{X}\right)$ & $\operatorname{vec}(\mathbf{A})$ \\
\hline $\mathbf{Z}=\mathbf{Z}(\mathbf{Y}), \mathbf{Y}=\mathbf{Y}(\mathbf{X})$ & $\frac{d \mathbf{Z}}{d \mathbf{X}}=\frac{d \mathbf{Y}}{d \mathbf{X}} \frac{d \mathbf{Z}}{d \mathbf{Y}}$ \\
\hline $\mathbf{Y}=\mathbf{A X B}$ & $\frac{d \mathbf{Y}}{d \mathbf{X}}=\mathbf{B} \otimes \mathbf{A}^{\top}$ \\
\hline $\mathbf{Z}=\mathbf{A Y B}$ & $\frac{d \mathbf{Z}}{d \mathbf{X}}=\frac{d \mathbf{Y}}{d \mathbf{X}}\left(\mathbf{B} \otimes \mathbf{A}^{\top}\right)$ \\
\hline $\mathbf{W}=\mathbf{Y Z Z}, \mathbf{Z} \in \mathbb{R}^{s \times t}$ & $\frac{d \mathbf{W}}{d \mathbf{X}}=\frac{d \mathbf{Y}}{d \mathbf{X}}\left(\mathbf{Z} \otimes \mathbf{I}_{r}^{\top}\right)+\frac{d \mathbf{Z}}{d \mathbf{X}}\left(\mathbf{I}_{t} \otimes \mathbf{Y}^{\top}\right)$ \\
\hline $\mathbf{W}=\mathbf{Y} \otimes \mathbf{Z}, \mathbf{Z} \in \mathbb{R}^{m \times n}$ & $\frac{d \mathbf{W}}{d \mathbf{X}}=\left[\frac{d \mathbf{Y}}{d \mathbf{X}} \otimes \operatorname{vec}(\mathbf{Z})^{\top}+\operatorname{vec}(\mathbf{Z})^{\top} \otimes \frac{d \mathbf{Y}}{d \mathbf{X}}\right]\left(\mathbf{I}_{s} \otimes \mathbf{K}_{r, n} \otimes \mathbf{I}_{m}\right)$ \\
\hline
\end{tabular}


Consider $\mathbf{X} \in \mathbb{R}^{p \times 1}, \mathbf{Y} \in \mathbb{k}^{r \times 1}$ and $z=z(\mathbf{X}) \in \mathbb{R} \mathbf{R}$, such that $\mathbf{X}=\mathbf{M Y}$ for $\mathbf{M} \in \mathbb{\mathbb { R } ^ { p \times r }}$

$$
\begin{aligned}
\frac{\partial z}{\partial \mathbf{Y}} & =\frac{\partial \mathbf{X}}{\partial \mathbf{Y}} \frac{\partial z}{\partial \mathbf{X}} \\
& =\frac{\partial\left(\mathbf{M Y} \mathbf{I}_{1}\right)}{\partial \mathbf{Y}} \frac{\partial z}{\partial \mathbf{X}} \\
& =\mathbf{M}^{\top} \frac{\partial z}{\partial \mathbf{X}} \\
\frac{\partial}{\partial \mathbf{Y}}\left\{\frac{\partial z}{\partial \mathbf{Y}}\right\}^{\top} & =\frac{\partial \mathbf{X}}{\partial \mathbf{Y}} \frac{\partial}{\partial \mathbf{X}}\left\{\frac{\partial z}{\partial \mathbf{Y}}\right\}^{\top} \\
& =\frac{\partial \mathbf{X}}{\partial \mathbf{Y}} \frac{\partial}{\partial \mathbf{X}}\left(\mathbf{I}_{1}\left\{\frac{\partial z}{\partial \mathbf{X}}\right\}^{\top} \mathbf{M}\right) \\
& =\mathbf{M}^{\top}\left(\frac{\partial}{\partial \mathbf{X}}\left\{\frac{\partial z}{\partial \mathbf{X}}\right\}^{\top}\right)^{\mathbf{M}} \\
& =\mathbf{M}^{\top} \frac{\partial^{2} z}{\partial \mathbf{X} \partial \mathbf{X}^{\top}} \mathbf{M}
\end{aligned}
$$




\section{Appendix B}

\section{Complete data information matrix}

Our goal is obtain expressions for the complete data log-likelihood when the random effects have a normal distribution. The log-likelihood has the following expression.

$$
\log L_{c}\left(\boldsymbol{\psi} ; \mathbf{u}, \mathbf{y}^{*}\right)=-\frac{1}{2} \sum_{i=1}^{K} \sum_{j=1}^{n_{2}} \log \left|\boldsymbol{\Sigma}_{e}\right|-\frac{1}{2} \sum_{i=1}^{K} \sum_{j=1}^{n_{i}} \boldsymbol{\epsilon}_{i j}^{\top} \boldsymbol{\Sigma}_{e}^{-1} \boldsymbol{\epsilon}_{i j}-\frac{1}{2} \sum_{i=1}^{K} \log |\boldsymbol{\Sigma}|-\frac{1}{2} \sum_{i=1}^{K} \mathbf{u}_{i}^{\top} \boldsymbol{\Sigma}^{-1} \mathbf{u}_{i}
$$

where

$$
\boldsymbol{\epsilon}_{i j}(\boldsymbol{\beta}):=\mathbf{y}_{i j}^{*}-\mathbf{x}_{i j} \boldsymbol{\beta}-\mathbf{z}_{i j} \mathbf{u}_{i}
$$

For convenience define

$$
A=\log \left|\boldsymbol{\Sigma}_{e}\right|, \quad B_{i j}=\boldsymbol{\epsilon}_{i j}^{\top} \boldsymbol{\Sigma}_{e}^{-1} \boldsymbol{\epsilon}_{i j}, \quad C=\log |\boldsymbol{\Sigma}|, \quad D_{i}=\mathbf{b}_{i}^{\top} \boldsymbol{\Sigma}^{-1} \mathbf{b}_{i}
$$

The matrices $\boldsymbol{\Sigma}_{e}$ is an $r \times r$ matrix, $\boldsymbol{\beta}$ is a $q \times 1$ matrix, $\mathbf{u}_{i}$ is an $s \times 1$ matrix, and $\boldsymbol{\Sigma}$ is an $s \times s$ matrix. 


$$
\begin{aligned}
\frac{\partial A}{\partial \boldsymbol{\Sigma}_{e}} & =\frac{\partial \log \left|\boldsymbol{\Sigma}_{e}\right|}{\partial \boldsymbol{\Sigma}_{e}} \\
& =\left(\frac{\partial \log \left|\boldsymbol{\Sigma}_{e}\right|}{\partial\left|\boldsymbol{\Sigma}_{e}\right|}\right)\left(\frac{\partial\left|\boldsymbol{\Sigma}_{e}\right|}{\partial \boldsymbol{\Sigma}_{e}}\right) \\
& =\left(\left|\boldsymbol{\Sigma}_{e}\right|^{-1}\right)\left(\left|\boldsymbol{\Sigma}_{e}\right| \operatorname{vec}\left(\boldsymbol{\Sigma}_{e}^{-1}\right)^{\top}\right) \\
& =\operatorname{vec}\left(\left\{\boldsymbol{\Sigma}_{e}^{-1}\right\}^{\top}\right) \\
& \frac{\partial A}{\partial \boldsymbol{\Sigma}_{e}}=\operatorname{vec}\left(\left\{\boldsymbol{\Sigma}_{e}^{-1}\right\}^{\top}\right)
\end{aligned}
$$

In a similar manner

$$
\begin{gathered}
\frac{\partial C}{\partial \boldsymbol{\Sigma}^{\top}}=\operatorname{vec}\left(\left\{\boldsymbol{\Sigma}^{-1}\right\}^{\top}\right) \\
\frac{\partial D_{i}}{\partial \boldsymbol{\Sigma}}=\frac{\partial}{\partial \boldsymbol{\Sigma}}\left(\mathbf{u}_{i}^{\top} \boldsymbol{\Sigma}^{-1} \mathbf{u}_{i}\right) \\
=\left(\frac{\partial \boldsymbol{\Sigma}^{-1}}{\partial \boldsymbol{\Sigma}}\right)\left(\mathbf{u}_{i} \otimes \mathbf{u}_{i}\right) \\
=\left[-\boldsymbol{\Sigma}^{-1} \otimes\left(\boldsymbol{\Sigma}^{\top}\right)^{-1}\right]\left(\mathbf{u}_{i} \otimes \mathbf{u}_{i}\right)
\end{gathered}
$$

Hence

$$
\begin{aligned}
\frac{\partial D_{i}}{\partial \boldsymbol{\Sigma}} & =-\left[\boldsymbol{\Sigma}^{-1} \otimes\left(\boldsymbol{\Sigma}^{\top}\right)^{-1}\right]\left(\mathbf{u}_{i} \otimes \mathbf{u}_{i}\right) \\
\frac{\partial B_{i j}}{\partial \boldsymbol{\Sigma}_{e}} & =\frac{\partial}{\partial \boldsymbol{\Sigma}_{e}}\left\{\boldsymbol{\epsilon}_{i j}^{\top} \boldsymbol{\Sigma}_{e}^{-1} \boldsymbol{\epsilon}_{i j}\right\} \\
& =\frac{\partial \boldsymbol{\Sigma}_{e}^{-1}}{\partial \boldsymbol{\Sigma}_{e}}\left[\boldsymbol{\epsilon}_{i j} \otimes\left(\boldsymbol{\epsilon}_{i j}^{\top}\right)^{\top}\right] \\
& =\left(\frac{\partial \boldsymbol{\Sigma}_{e}^{-1}}{\partial \boldsymbol{\Sigma}_{e}}\right)\left(\boldsymbol{\epsilon}_{i j} \otimes \boldsymbol{\epsilon}_{i j}\right) \\
& =-\left[\boldsymbol{\Sigma}_{e}^{-1} \otimes\left(\boldsymbol{\Sigma}_{e}^{\top}\right)^{-1}\right]\left(\boldsymbol{\epsilon}_{i j} \otimes \boldsymbol{\epsilon}_{i j}\right)
\end{aligned}
$$


Hence

$$
\frac{\partial B_{i j}}{\partial \boldsymbol{\Sigma}_{e}}=-\left[\boldsymbol{\Sigma}_{e}^{-1} \otimes\left(\boldsymbol{\Sigma}_{e}^{\top}\right)^{-1}\right]\left(\boldsymbol{\epsilon}_{i j} \otimes \boldsymbol{\epsilon}_{i j}\right)
$$

$$
\begin{aligned}
\frac{\partial B_{i j}}{\partial \boldsymbol{\beta}} & =\frac{\partial}{\partial \boldsymbol{\beta}}\left\{\boldsymbol{\epsilon}_{i j}^{\top} \boldsymbol{\Sigma}_{e}^{-1} \boldsymbol{\epsilon}_{i j}\right\} \\
& =\left\{\frac{\partial \boldsymbol{\epsilon}_{i j}^{\top}}{\partial \boldsymbol{\beta}}\right\}\left[\left(\boldsymbol{\Sigma}_{e}^{-1} \boldsymbol{\epsilon}_{i j}\right) \otimes \mathbf{I}_{\mathbf{1}}\right]+\left\{\frac{\partial}{\partial \boldsymbol{\beta}}\left(\boldsymbol{\Sigma}_{e}^{-1} \boldsymbol{\epsilon}_{i j}\right)\right\}\left[\mathbf{I}_{1} \otimes\left(\boldsymbol{\epsilon}_{i j}^{\top}\right)^{\top}\right] \\
& =\left\{\frac{\partial \boldsymbol{\epsilon}_{i j}^{\top}}{\partial \boldsymbol{\beta}}\right\}\left(\boldsymbol{\Sigma}_{e}^{-1} \boldsymbol{\epsilon}_{i j}\right)+\left\{\frac{\partial}{\partial \boldsymbol{\beta}}\left(\boldsymbol{\Sigma}_{e}^{-1} \boldsymbol{\epsilon}_{i j} \mathbf{I}_{1}\right)\right\} \boldsymbol{\epsilon}_{i j} \\
& =\left\{\frac{\partial \boldsymbol{\epsilon}_{i j}^{\top}}{\partial \boldsymbol{\beta}}\right\} \boldsymbol{\Sigma}_{e}^{-1} \boldsymbol{\epsilon}_{i j}+\left\{\frac{\partial \boldsymbol{\epsilon}_{i j}}{\partial \boldsymbol{\beta}}\right\}\left(\boldsymbol{\Sigma}_{e}^{-1}\right)^{\top} \boldsymbol{\epsilon}_{i j}
\end{aligned}
$$

As a result

$$
\frac{\partial B_{i j}}{\partial \boldsymbol{\beta}}=\left\{\frac{\partial \boldsymbol{\epsilon}_{i j}^{\top}}{\partial \boldsymbol{\beta}}\right\} \boldsymbol{\Sigma}_{e}^{-1} \boldsymbol{\epsilon}_{i j}+\left\{\frac{\partial \boldsymbol{\epsilon}_{i j}}{\partial \boldsymbol{\beta}}\right\}\left(\boldsymbol{\Sigma}_{\boldsymbol{e}}^{-1}\right)^{\top} \boldsymbol{\epsilon}_{i j}
$$

We have $\boldsymbol{\epsilon}_{i j}=\mathbf{y}_{i_{-j}}^{*}-\mathbf{x}_{i_{-} j} \boldsymbol{\beta}-\mathbf{z}_{i_{j}} \mathbf{i}$.

$$
\begin{aligned}
\frac{\partial \boldsymbol{\epsilon}_{i j}}{\partial \boldsymbol{\beta}} & =-\frac{\partial\left(\mathbf{x}_{i-j} \boldsymbol{\beta}\right)}{\partial \boldsymbol{\beta}} \\
& =-\left(\frac{\partial \boldsymbol{\beta}}{\partial \boldsymbol{\beta}}\right)\left(\mathbf{I}_{1} \otimes \mathbf{x}_{i_{-j}}^{\top}\right) \\
& =-\mathbf{x}_{i_{-j}}^{\top}
\end{aligned}
$$

Hence

$$
\frac{\partial \boldsymbol{\epsilon}_{i j}}{\partial \boldsymbol{\beta}}=-\mathbf{x}_{i-j}^{\top}
$$

Also

$$
\begin{aligned}
\frac{\partial \boldsymbol{\epsilon}_{i j}^{\top}}{\partial \boldsymbol{\beta}} & =-\frac{\partial\left(\boldsymbol{\beta}^{\top} \mathbf{x}_{i-j}^{\top}\right)}{\partial \boldsymbol{\beta}} \\
& =-\frac{\partial \boldsymbol{\beta}^{\top}}{\partial \boldsymbol{\beta}} \frac{\partial\left(\boldsymbol{\beta}^{\top} \mathbf{x}_{i-j}^{\top}\right)}{\partial\left(\boldsymbol{\beta}^{\top}\right)} \\
& =-\mathbf{x}_{i-j}^{\top}
\end{aligned}
$$


Hence

$$
\frac{\partial \boldsymbol{\epsilon}_{i j}^{\top}}{\partial \boldsymbol{\beta}}=-\mathbf{x}_{i-j}^{\top}
$$

As a result

$$
\frac{\partial B_{i j}}{\partial \boldsymbol{\beta}}=-\mathbf{x}_{i_{-j}}^{\top}\left[\boldsymbol{\Sigma}_{e}^{-1}+\left(\boldsymbol{\Sigma}_{e}^{-1}\right)^{\top}\right] \boldsymbol{\epsilon}_{i j}
$$

Therefore the score equations are as follows:

$$
\begin{aligned}
& \frac{\partial \log L_{c}}{\partial \boldsymbol{\beta}}=\sum_{i=1}^{K} \sum_{j=1}^{n_{i}} \mathbf{x}_{i j}^{\top} \boldsymbol{\Sigma}_{e}^{-1} \boldsymbol{\epsilon}_{i j} \\
& \frac{\partial \log L_{c}}{\partial \boldsymbol{\Sigma}}=-\frac{1}{2} \sum_{i=1}^{n}\left\{\operatorname{vec}\left(\left\{\boldsymbol{\Sigma}^{-1}\right\}^{\top}\right)-\left(\boldsymbol{\Sigma}^{-1} \otimes \boldsymbol{\Sigma}^{-1}\right)\left(\mathbf{u}_{i} \otimes \mathbf{u}_{i}\right)\right\} \\
& \frac{\partial \log L_{c}}{\partial \boldsymbol{\Sigma}_{e}}=-\frac{1}{2} \sum_{i=1}^{K} \sum_{j=1}^{n_{i}}\left\{\operatorname{vec}\left(\left\{\boldsymbol{\Sigma}_{e}^{-1}\right\}^{\top}\right)-\left(\boldsymbol{\Sigma}_{e}^{-1} \otimes \boldsymbol{\Sigma}_{e}^{-1}\right)\left(\boldsymbol{\epsilon}_{i j} \otimes \boldsymbol{\epsilon}_{i j}\right)\right\}
\end{aligned}
$$

The matrix $\operatorname{vec}\left(\boldsymbol{\Sigma}_{e}^{-1}\right)$ is an $r^{2} \times 1$ matrix

$$
\begin{aligned}
& \frac{\partial^{2} A}{\partial \boldsymbol{\Sigma}_{e} \partial \boldsymbol{\Sigma}_{e}^{\top}}=\frac{\partial}{\partial \boldsymbol{\Sigma}_{e}}\left(\frac{\partial A}{\partial \boldsymbol{\Sigma}}\right)^{\top} \\
&=-\boldsymbol{\Sigma}_{e}^{-1} \otimes\left(\boldsymbol{\Sigma}_{e}^{\top}\right)^{-1} \\
& \frac{\partial^{2} A}{\partial \boldsymbol{\Sigma}_{e} \partial \boldsymbol{\Sigma}_{e}^{\top}}=-\boldsymbol{\Sigma}_{e}^{-1} \otimes\left(\boldsymbol{\Sigma}_{e}^{\top}\right)^{-1}=-\boldsymbol{\Sigma}_{e}^{-1} \otimes \boldsymbol{\Sigma}_{e}^{-1}
\end{aligned}
$$

In a similar manner

$$
\frac{\partial^{2} C}{\partial \boldsymbol{\Sigma} \partial \boldsymbol{\Sigma}^{\top}}=-\boldsymbol{\Sigma}^{-1} \otimes \boldsymbol{\Sigma}^{-1}
$$




$$
\begin{aligned}
\frac{\partial^{2} D_{i}}{\partial \boldsymbol{\Sigma} \partial \boldsymbol{\Sigma}^{\top}} & =\frac{\partial}{\partial \boldsymbol{\Sigma}}\left(\frac{\partial D_{i}}{\partial \boldsymbol{\Sigma}}\right)^{\top} \\
& =\frac{\partial}{\partial \boldsymbol{\Sigma}}\left\{-\left[\boldsymbol{\Sigma}^{-1} \otimes\left(\boldsymbol{\Sigma}^{\top}\right)^{-1}\right]\left(\mathbf{u}_{i} \otimes \mathbf{u}_{i}\right)\right\}^{\top} \\
& =-\frac{\partial}{\partial \boldsymbol{\Sigma}}\left\{\left(\mathbf{u}_{i} \otimes \mathbf{u}_{i}\right)^{\top}\left[\boldsymbol{\Sigma}^{-1} \otimes\left(\boldsymbol{\Sigma}^{\top}\right)^{-1}\right]^{\top}\right\} \\
& =-\frac{\partial}{\partial \boldsymbol{\Sigma}}\left\{\left(\mathbf{u}_{i} \otimes \mathbf{u}_{i}\right)^{\top}\left[\left(\boldsymbol{\Sigma}^{\top}\right)^{-1} \otimes \boldsymbol{\Sigma}^{-1}\right] \mathbf{I}_{s^{2}}\right\} \\
& =-\left(\frac{\partial}{\partial \boldsymbol{\Sigma}}\left[\left(\boldsymbol{\Sigma}^{\top}\right)^{-1} \otimes \boldsymbol{\Sigma}^{-1}\right]\right)\left[\mathbf{I}_{s^{2}} \otimes\left(\mathbf{u}_{i} \otimes \mathbf{u}_{i}\right)\right] \\
\frac{\partial^{2} D_{i}}{\partial \boldsymbol{\Sigma} \partial \boldsymbol{\Sigma}^{\top}} & =-\left(\frac{\partial}{\partial \boldsymbol{\Sigma}}\left[\left(\boldsymbol{\Sigma}^{\top}\right)^{-1} \otimes \boldsymbol{\Sigma}^{-1}\right]\right)\left(\mathbf{I}_{s^{2}} \otimes \mathbf{u}_{i} \otimes \mathbf{u}_{i}\right)
\end{aligned}
$$

However

$$
\begin{aligned}
& \frac{\partial}{\partial \boldsymbol{\Sigma}}\left[\left(\boldsymbol{\Sigma}^{\top}\right)^{-1} \otimes \boldsymbol{\Sigma}^{-1}\right]= \\
& {\left[\frac{\partial\left(\boldsymbol{\Sigma}^{\top}\right)^{-1}}{\partial \boldsymbol{\Sigma}} \otimes\left\{\operatorname{vec}\left(\boldsymbol{\Sigma}^{-1}\right)\right\}^{\top}+\left\{\operatorname{vec}\left(\left(\boldsymbol{\Sigma}^{\top}\right)^{-1}\right)\right\}^{\top} \otimes \frac{\partial \boldsymbol{\Sigma}^{-1}}{\partial \boldsymbol{\Sigma}}\right]\left(\mathbf{I}_{s} \otimes \mathbf{K}_{s, \boldsymbol{s}} \otimes \mathbf{I}_{s}\right)(\text { B.46) }}
\end{aligned}
$$

Therefore

$$
\frac{\partial}{\partial \boldsymbol{\Sigma}}\left[\boldsymbol{\Sigma}^{-1} \otimes\left(\boldsymbol{\Sigma}^{\top}\right)^{-1}\right]=\left[\frac{\partial\left(\boldsymbol{\Sigma}^{\top}\right)^{-1}}{\partial \boldsymbol{\Sigma}} \otimes\left\{\operatorname{vec}\left(\boldsymbol{\Sigma}^{-1}\right)\right\}^{\top}+\left\{\operatorname{vec}\left(\left(\boldsymbol{\Sigma}^{\top}\right)^{-1}\right)\right\}^{\top} \otimes \frac{\partial \boldsymbol{\Sigma}^{-1}}{\partial \boldsymbol{\Sigma}}\right]\left(\mathbf{I}_{s} \otimes \mathbf{K}_{s, s} \otimes \mathbf{I}_{s}\right)
$$

Also

$$
\begin{aligned}
\frac{\partial \boldsymbol{\Sigma}^{-1}}{\partial \boldsymbol{\Sigma}} & =-\boldsymbol{\Sigma}^{-1} \otimes\left(\boldsymbol{\Sigma}^{\top}\right)^{-1} \\
\frac{\partial\left(\boldsymbol{\Sigma}^{\top}\right)^{-1}}{\partial \boldsymbol{\Sigma}} & =\left(\frac{\partial \boldsymbol{\Sigma}^{\top}}{\partial \boldsymbol{\Sigma}}\right)\left[\frac{\partial\left(\boldsymbol{\Sigma}^{\top}\right)^{-1}}{\partial \boldsymbol{\Sigma}^{\top}}\right] \\
& =-\mathbf{K}_{s, s}\left[\left(\boldsymbol{\Sigma}^{\top}\right)^{-1} \otimes\left(\left(\boldsymbol{\Sigma}^{\top}\right)^{\top}\right)^{-1}\right] \\
& =-\mathbf{K}_{s, s}\left[\left(\boldsymbol{\Sigma}^{\top}\right)^{-1} \otimes \boldsymbol{\Sigma}^{-1}\right]
\end{aligned}
$$




$$
\begin{aligned}
\frac{\partial}{\partial \boldsymbol{\Sigma}}\left[\boldsymbol{\Sigma}^{-1} \otimes\left(\boldsymbol{\Sigma}^{\top}\right)^{-1}\right]= & -\left[\left\{\mathbf{K}_{s, s}\left[\left(\boldsymbol{\Sigma}^{\top}\right)^{-1} \otimes \boldsymbol{\Sigma}^{-1}\right]\right\} \otimes\left\{\operatorname{vec}\left(\boldsymbol{\Sigma}^{-1}\right)\right\}^{\top}+\right. \\
& \left.\left\{\operatorname{vec}\left(\left(\boldsymbol{\Sigma}^{\top}\right)^{-1}\right)\right\}^{\top} \otimes\left(\boldsymbol{\Sigma}^{-1} \otimes\left(\boldsymbol{\Sigma}^{\top}\right)^{-1}\right)\right]\left(\mathbf{I}_{s} \otimes \mathbf{K}_{s, s} \otimes \mathbf{I}_{s}\right)
\end{aligned}
$$

As a result

$$
\begin{aligned}
\frac{\partial^{2} D_{i}}{\partial \boldsymbol{\Sigma} \partial \boldsymbol{\Sigma}^{\top}}= & {\left[\left\{\mathbf{K}_{s, s}\left[\left(\boldsymbol{\Sigma}^{\top}\right)^{-1} \otimes \boldsymbol{\Sigma}^{-1}\right]\right\} \otimes\left\{\operatorname{vec}\left(\boldsymbol{\Sigma}^{-1}\right)\right\}^{\top}+\right.} \\
& \left.\left\{\operatorname{vec}\left(\left(\boldsymbol{\Sigma}^{\top}\right)^{-1}\right)\right\}^{\top} \otimes\left(\boldsymbol{\Sigma}^{-1} \otimes\left(\boldsymbol{\Sigma}^{\top}\right)^{-1}\right)\right]\left(\mathbf{I}_{s} \otimes \mathbf{K}_{s, s} \otimes \mathbf{I}_{s}\right)\left(\mathbf{I}_{s^{2}} \otimes \mathbf{u}_{i} \otimes \mathbf{u}_{i}\right)
\end{aligned}
$$

$$
\begin{aligned}
\frac{\partial^{2} B_{i j}}{\partial \boldsymbol{\beta} \partial \boldsymbol{\beta}^{\top}} & =\frac{\partial}{\partial \boldsymbol{\beta}}\left\{-\mathbf{x}_{i_{-} j}^{\top}\left[\boldsymbol{\Sigma}_{e}^{-1}+\left(\boldsymbol{\Sigma}_{e}^{-1}\right)^{\top}\right] \boldsymbol{\epsilon}_{i j}\right\}^{\top} \\
& =-\left\{\frac{\partial \boldsymbol{\epsilon}_{i j}}{\partial \boldsymbol{\beta}}\right\}\left\{\mathbf{I}_{1} \otimes\left(\mathbf{x}_{i_{-j}}^{\top}\left[\boldsymbol{\Sigma}_{e}^{-1}+\left(\boldsymbol{\Sigma}_{e}^{-1}\right)^{\top}\right]\right)^{\top}\right\} \\
& =\mathbf{x}_{i_{-j}}^{\top}\left[\boldsymbol{\Sigma}_{e}^{-1}+\left(\boldsymbol{\Sigma}_{e}^{-1}\right)^{\top}\right] \mathbf{x}_{i_{-j}}
\end{aligned}
$$$$
\frac{\partial^{2} B_{i j}}{\partial \boldsymbol{\beta} \partial \boldsymbol{\beta}^{\top}}=\mathbf{x}_{i_{-j}}^{\top}\left[\boldsymbol{\Sigma}_{e}^{-1}+\left(\boldsymbol{\Sigma}_{e}^{-1}\right)^{\top}\right] \mathbf{x}_{i_{-j}}=2 \mathbf{x}_{i_{-j}}^{\top} \boldsymbol{\Sigma}_{e}^{-1} \mathbf{x}_{i_{-j}}
$$

$$
\begin{aligned}
\frac{\partial^{2} B_{i j}}{\partial \boldsymbol{\Sigma}_{e} \partial \boldsymbol{\Sigma}_{e}^{\top}} & =\frac{\partial}{\partial \boldsymbol{\Sigma}_{e}}\left\{-\mathbf{I}_{r^{2}}\left[\boldsymbol{\Sigma}_{e}^{-1} \otimes\left(\boldsymbol{\Sigma}_{e}^{\top}\right)^{-1}\right]\left(\boldsymbol{\epsilon}_{i j} \otimes \boldsymbol{\epsilon}_{i j}\right)\right\}^{\top} \\
& =-\left\{\frac{\partial}{\partial \boldsymbol{\Sigma}_{e}}\left[\boldsymbol{\Sigma}_{e}^{-1} \otimes\left(\boldsymbol{\Sigma}_{e}^{\top}\right)^{-1}\right]\right\}\left(\boldsymbol{\epsilon}_{i j} \otimes \boldsymbol{\epsilon}_{i j} \otimes \mathbf{I}_{r^{2}}\right)
\end{aligned}
$$


We have

$$
\begin{aligned}
\frac{\partial}{\partial \boldsymbol{\Sigma}_{e}}\left[\boldsymbol{\Sigma}_{e}^{-1} \otimes\left(\boldsymbol{\Sigma}_{e}^{\top}\right)^{-1}\right]= & -\left[\left\{\mathbf{K}_{r, r}\left[\left(\boldsymbol{\Sigma}_{e}^{\top}\right)^{-1} \otimes \boldsymbol{\Sigma}_{e}^{-1}\right]\right\} \otimes\left\{\operatorname{vec}\left(\boldsymbol{\Sigma}_{e}^{-1}\right)\right\}^{\top}+\right. \\
& \left.\left\{\operatorname{vec}\left(\left(\boldsymbol{\Sigma}_{e}^{\top}\right)^{-1}\right)\right\}^{\top} \otimes\left(\boldsymbol{\Sigma}_{e}^{-1} \otimes\left(\boldsymbol{\Sigma}_{e}^{\top}\right)^{-1}\right)\right]\left(\mathbf{I}_{r} \otimes \mathbf{K}_{r, r} \otimes \mathbf{I}_{r}\right)
\end{aligned}
$$

Therefore

$$
\begin{aligned}
\frac{\partial^{2} B_{i j}}{\partial \boldsymbol{\Sigma}_{e} \partial \boldsymbol{\Sigma}_{e}^{\top}}= & -\left[\left\{\mathbf{K}_{r, r}\left[\left(\boldsymbol{\Sigma}_{e}^{\top}\right)^{-1} \otimes \boldsymbol{\Sigma}_{e}^{-1}\right]\right\} \otimes\left\{\operatorname{vec}\left(\boldsymbol{\Sigma}_{e}^{-1}\right)\right\}^{\top}+\right. \\
& \left.\left\{\operatorname{vec}\left(\left(\boldsymbol{\Sigma}_{e}^{\top}\right)^{-1}\right)\right\}^{\top} \otimes\left(\boldsymbol{\Sigma}_{e}^{-1} \otimes\left(\boldsymbol{\Sigma}_{e}^{\top}\right)^{-1}\right)\right]\left(\mathbf{I}_{r} \otimes \mathbf{K}_{r, r} \otimes \mathbf{I}_{r}\right)\left(\boldsymbol{\epsilon}_{i j} \otimes \boldsymbol{\epsilon}_{i j} \otimes \mathbf{I}_{r^{2}}\right)
\end{aligned}
$$

$$
\begin{aligned}
\frac{\partial^{2} B_{i j}}{\partial \boldsymbol{\Sigma}_{e} \partial \boldsymbol{\beta}^{\top}} & =\frac{\partial}{\partial \boldsymbol{\Sigma}_{e}}\left\{\frac{\partial B_{i j}}{\partial \boldsymbol{\beta}}\right\}^{\top} \\
& =\frac{\partial}{\partial \boldsymbol{\Sigma}_{e}}\left\{-\mathbf{x}_{i_{-}}^{\top}\left[\boldsymbol{\Sigma}_{e}^{-1}+\left(\boldsymbol{\Sigma}_{e}^{-1}\right)^{\top}\right] \boldsymbol{\epsilon}_{i j}\right\}^{\top} \\
& =-\left\{\frac{\partial}{\partial \boldsymbol{\Sigma}_{e}}\left[\left(\boldsymbol{\Sigma}_{e}^{-1}\right)^{\top}+\boldsymbol{\Sigma}_{e}^{-1}\right]\right\}\left(\mathbf{x}_{i-j}^{\top} \otimes \boldsymbol{\epsilon}_{i j}\right) \\
& =-\left[\frac{\partial \boldsymbol{\Sigma}_{e}^{-1}}{\partial \boldsymbol{\Sigma}_{e}} \frac{\left(\boldsymbol{\Sigma}_{e}^{-1}\right)^{\top}}{\partial \boldsymbol{\Sigma}_{e}^{-1}}+\frac{\partial \boldsymbol{\Sigma}_{e}^{-1}}{\partial \boldsymbol{\Sigma}_{e}}\right]\left(\mathbf{x}_{i-j}^{\top} \otimes \boldsymbol{\epsilon}_{i j}\right) \\
& =\left[\boldsymbol{\Sigma}_{e}^{-1} \otimes\left(\boldsymbol{\Sigma}_{e}^{\top}\right)^{-1}\right]\left(\mathbf{K}_{r, r}+\mathbf{I}_{r^{2}}\right)\left(\mathbf{x}_{i-j}^{\top} \otimes \boldsymbol{\epsilon}_{i j}\right)
\end{aligned}
$$

Hence

$$
\frac{\partial^{2} B_{i j}}{\partial \boldsymbol{\Sigma}_{e} \partial \boldsymbol{\beta}^{\top}}=\left[\boldsymbol{\Sigma}_{e}^{-1} \otimes\left(\boldsymbol{\Sigma}_{e}^{\top}\right)^{-1}\right]\left(\mathbf{K}_{r, r}+\mathbf{I}_{r^{2}}\right)\left(\mathbf{x}_{i_{-j}}^{\top} \otimes \boldsymbol{\epsilon}_{i j}\right)
$$


The equations for the observed information matrix are as follows.

$$
\begin{aligned}
& \frac{\partial^{2} \log L_{c}}{\partial \boldsymbol{\Sigma} \partial \boldsymbol{\Sigma}^{\top}}=\frac{1}{2} \sum_{i=1}^{K}\left\{\boldsymbol{\Sigma}^{-1} \otimes \boldsymbol{\Sigma}^{-1}-\right. \\
& {\left[\left\{\mathbf{K}_{s, s}\left(\boldsymbol{\Sigma}^{-1} \otimes \boldsymbol{\Sigma}^{-1}\right)\right\} \otimes\left\{\operatorname{vec}\left(\boldsymbol{\Sigma}^{-1}\right)\right\}^{\top}+\left\{\operatorname{vec}\left(\boldsymbol{\Sigma}^{-1}\right)\right\}^{\top} \otimes \boldsymbol{\Sigma}^{-1} \otimes \boldsymbol{\Sigma}^{-1}\right]\left(\mathbf{I}_{s} \otimes \mathbf{K}_{s, s} \otimes \mathbf{I}_{s}\right)} \\
& \left.\left[\operatorname{vec}\left(\mathbf{b}_{i} \mathbf{b}_{i}^{\top}\right) \otimes \mathbf{I}_{s^{2}}\right]\right\} \\
& \quad \frac{\partial^{2} \log L_{c}}{\partial \boldsymbol{\beta} \partial \boldsymbol{\beta}^{\top}}=-\sum_{i=1}^{K} \sum_{j=1}^{n_{i}} \mathbf{x}_{i j}^{\top} \boldsymbol{\Sigma}_{e}^{-1} \mathbf{x}_{i j} \\
& \frac{\partial^{2} \log L_{c}}{\partial \boldsymbol{\Sigma}_{e} \partial \boldsymbol{\Sigma}_{e}^{\top}}=\frac{1}{2} \sum_{i=1}^{K} \sum_{j=1}^{n_{i}}\left\{\boldsymbol{\Sigma}_{e}^{-1} \otimes \boldsymbol{\Sigma}_{e}^{-1}-\right. \\
& {\left[\left\{\mathbf{K}_{r, r}\left(\boldsymbol{\Sigma}_{e}^{-1} \otimes \boldsymbol{\Sigma}_{e}^{-1}\right)\right\} \otimes\left\{\operatorname{vec}\left(\boldsymbol{\Sigma}_{e}^{-1}\right)\right\}^{\top}+\left\{\operatorname{vec}\left(\boldsymbol{\Sigma}_{e}^{-1}\right)\right\}^{\top} \otimes\left(\boldsymbol{\Sigma}_{e}^{-1} \otimes \boldsymbol{\Sigma}_{e}^{-1}\right)\right]\left(\mathbf{I}_{r} \otimes \mathbf{K}_{r, r} \otimes \mathbf{I}_{r}\right)} \\
& \left.\left[\operatorname{vec}\left(\boldsymbol{e}_{i j} \boldsymbol{e}_{i j}^{\top}\right) \otimes \mathbf{I}_{r^{2}}\right]\right\}
\end{aligned}
$$

$$
\frac{\partial^{2} \log L_{c}}{\partial \boldsymbol{\Sigma}_{e} \partial \boldsymbol{\beta}_{e}^{\top}}=-\frac{1}{2}\left(\boldsymbol{\Sigma}_{e}^{-1} \otimes \boldsymbol{\Sigma}_{e}^{-1}\right)\left(\mathbf{K}_{r, r}+\mathbf{I}_{r^{2}}\right)\left(\sum_{i=1}^{K} \sum_{j=1}^{n_{i}} \mathbf{x}_{i j}^{\top} \otimes \boldsymbol{\epsilon}_{i j}\right)
$$




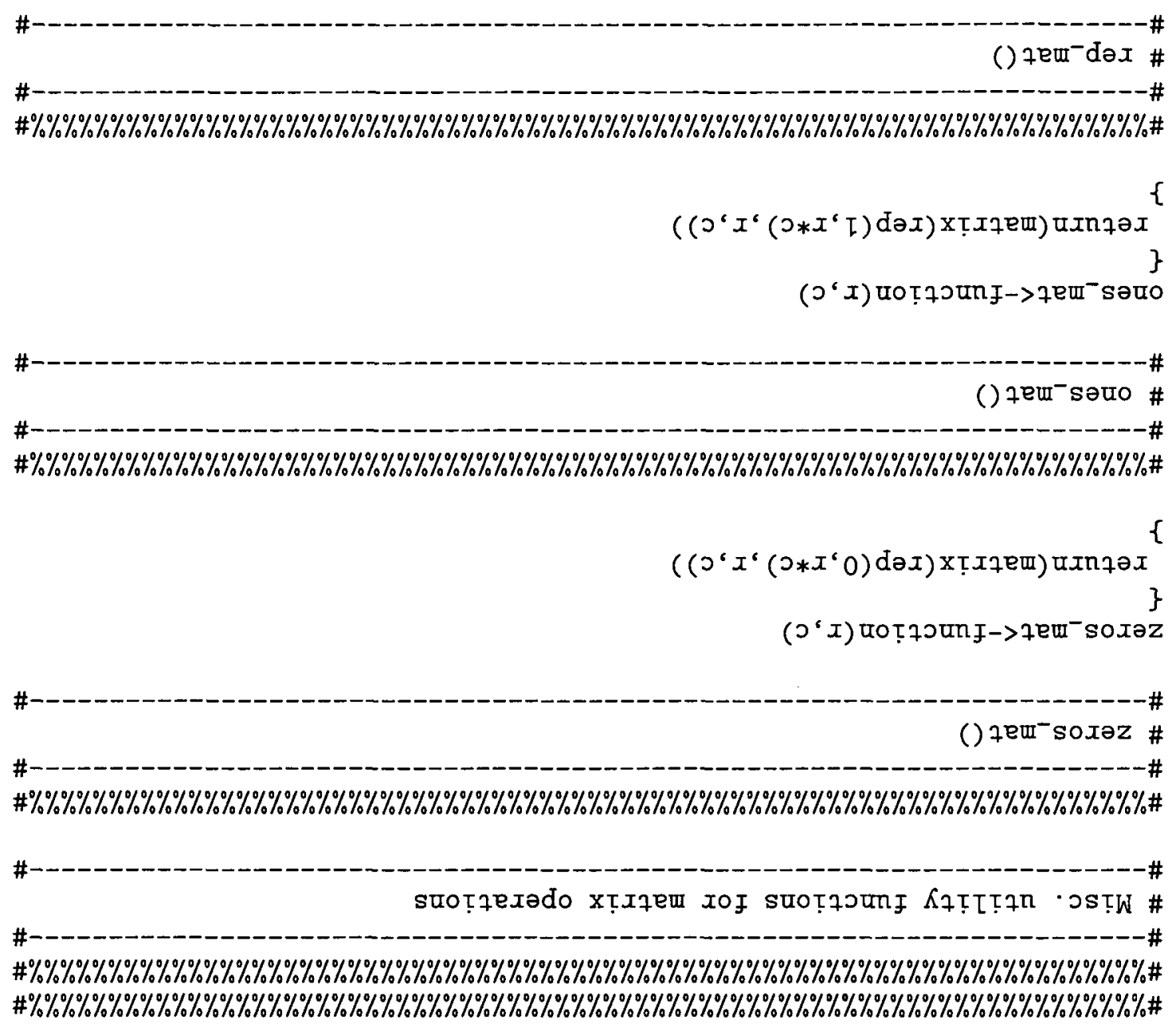

әроว $\boldsymbol{y}$

O x!puəddV 
\#-------\#\%\%\%\%\%\%\%\%\%\%\%\%\%\%\%\%\%\%\%\%\%\%\%\%\%\%\%\%\%\%\%\%\%\%\%\%\%\%\%\%\%\%\%\%\%\%\%\%\%\%\%\%\%\%\%\%\%\%\%\%\%\%\%\%\%\%\%\%\#

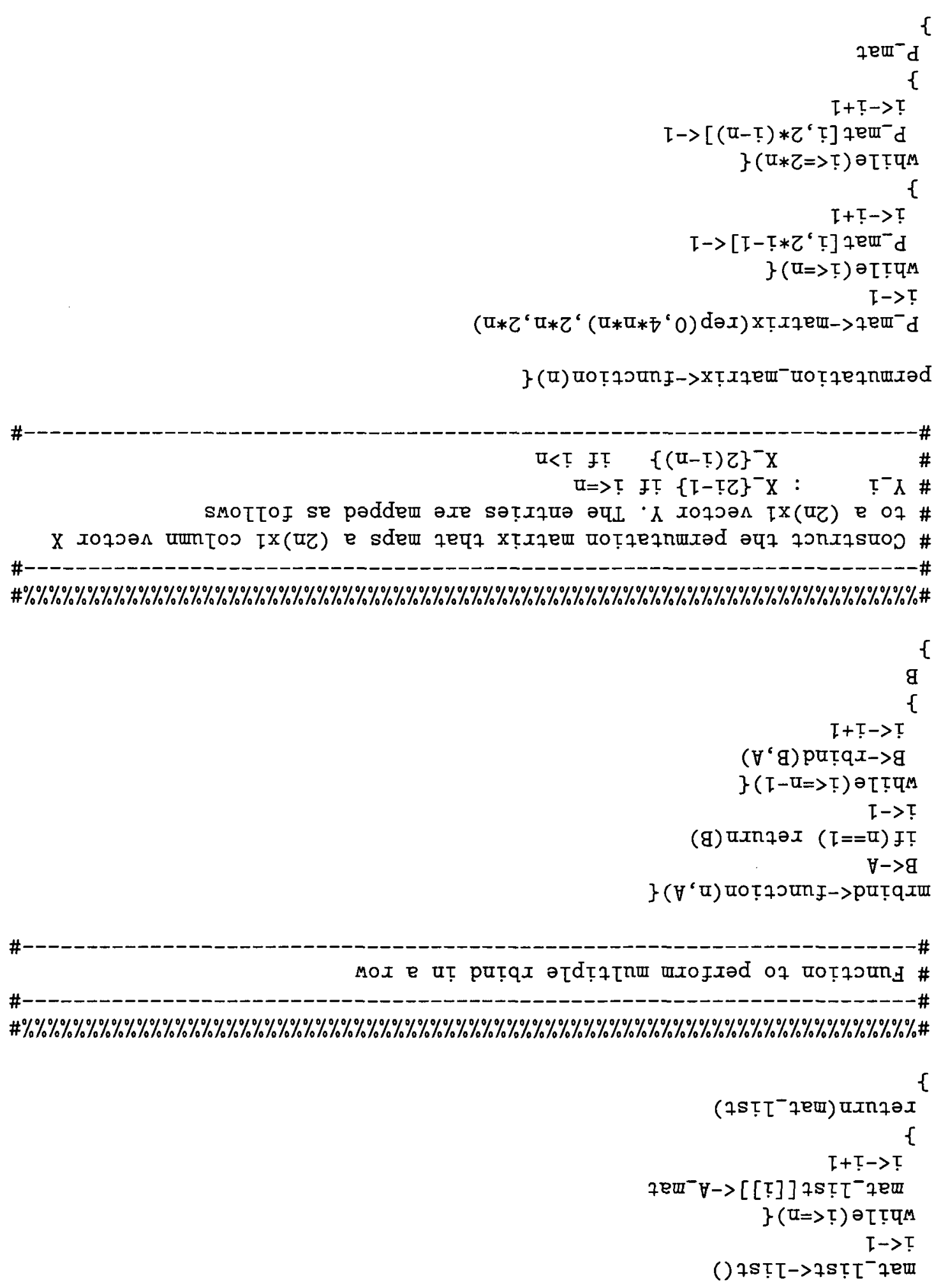


\# Apply permutation to mu_list

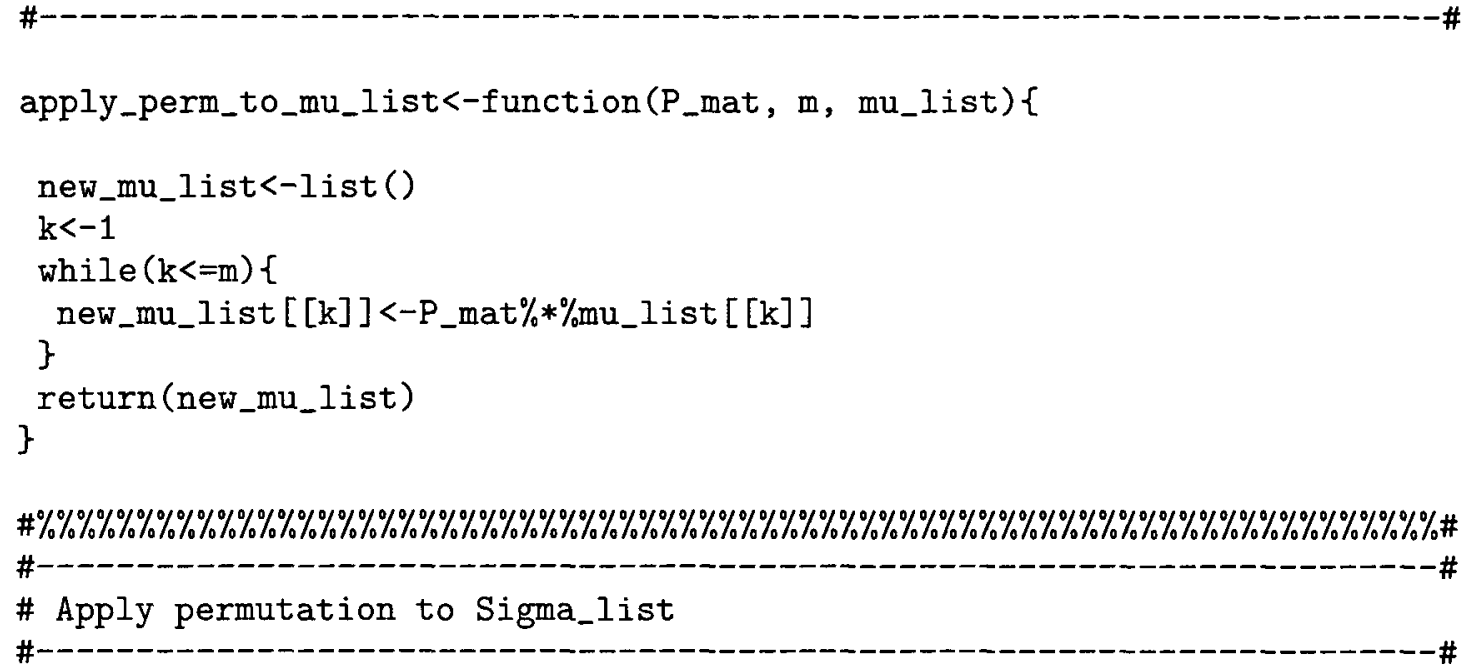

apply_perm_to_sigma_list<-function(P_mat, $m$, Sigma_list) \{

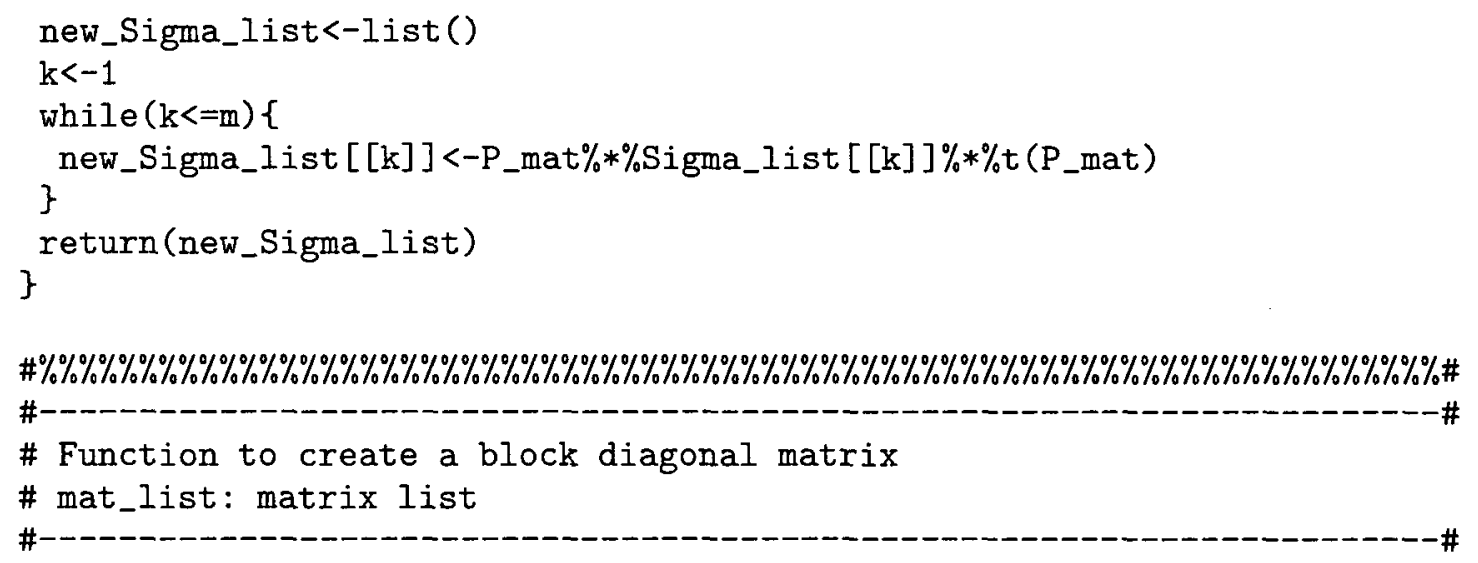




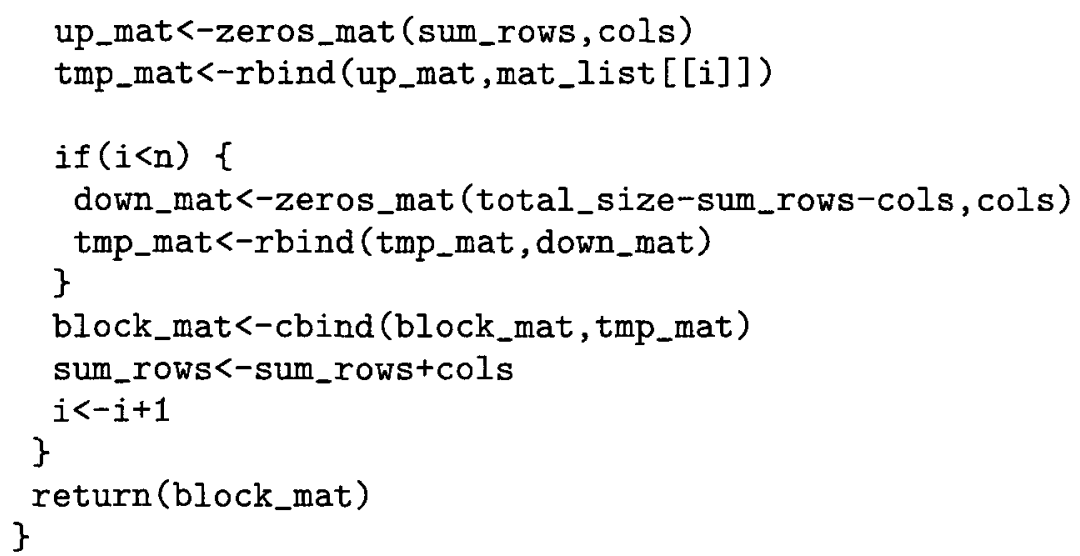




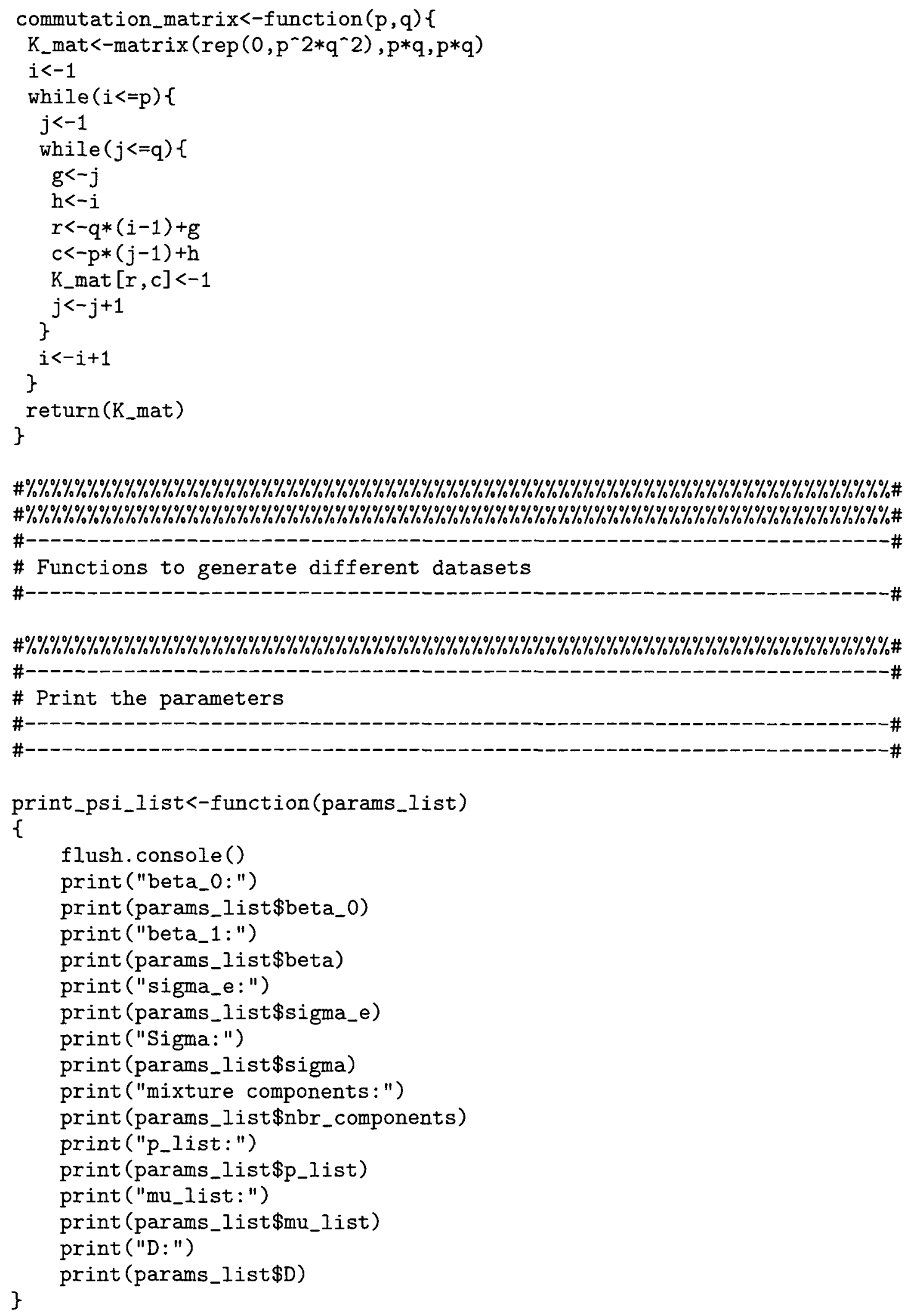




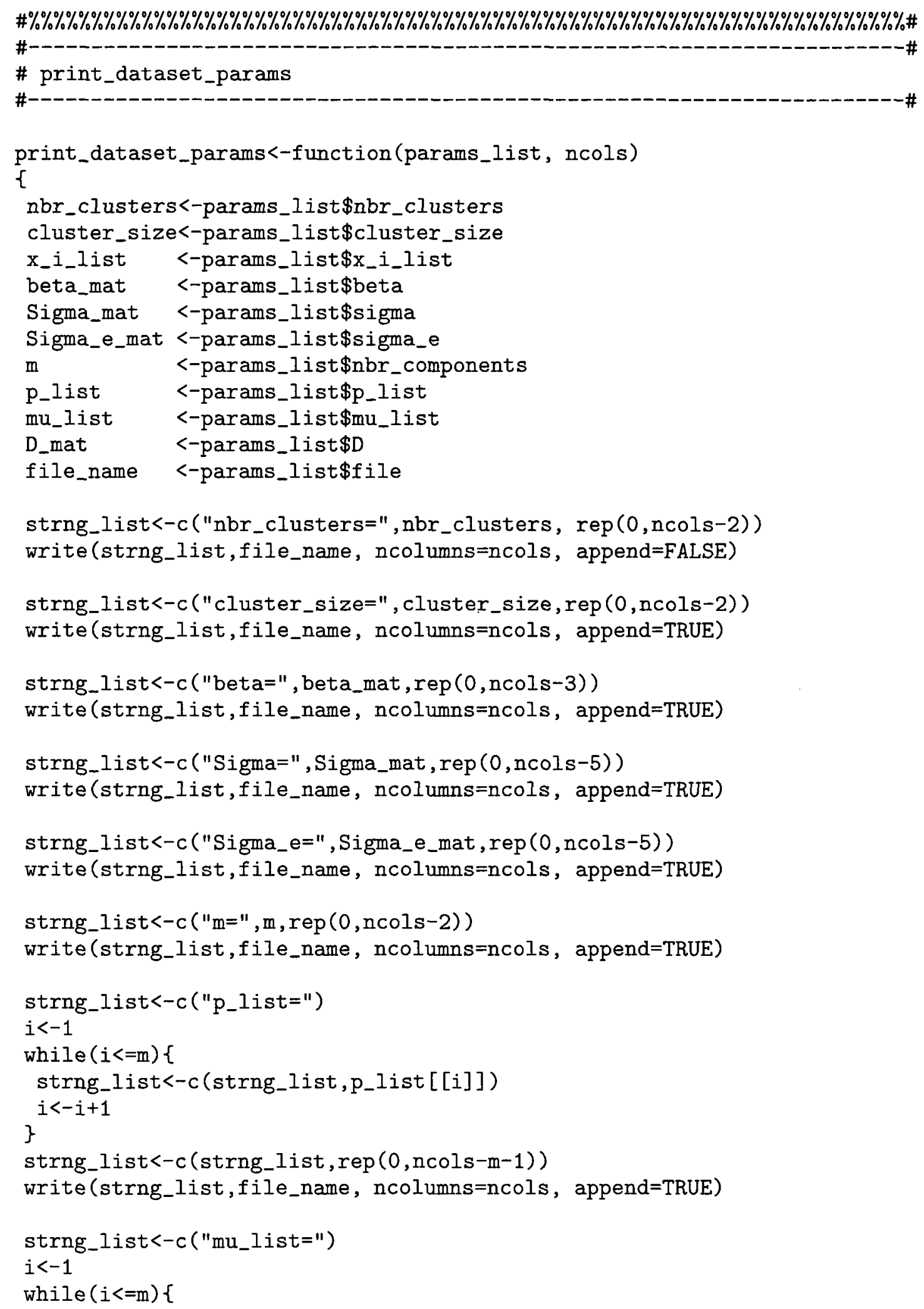




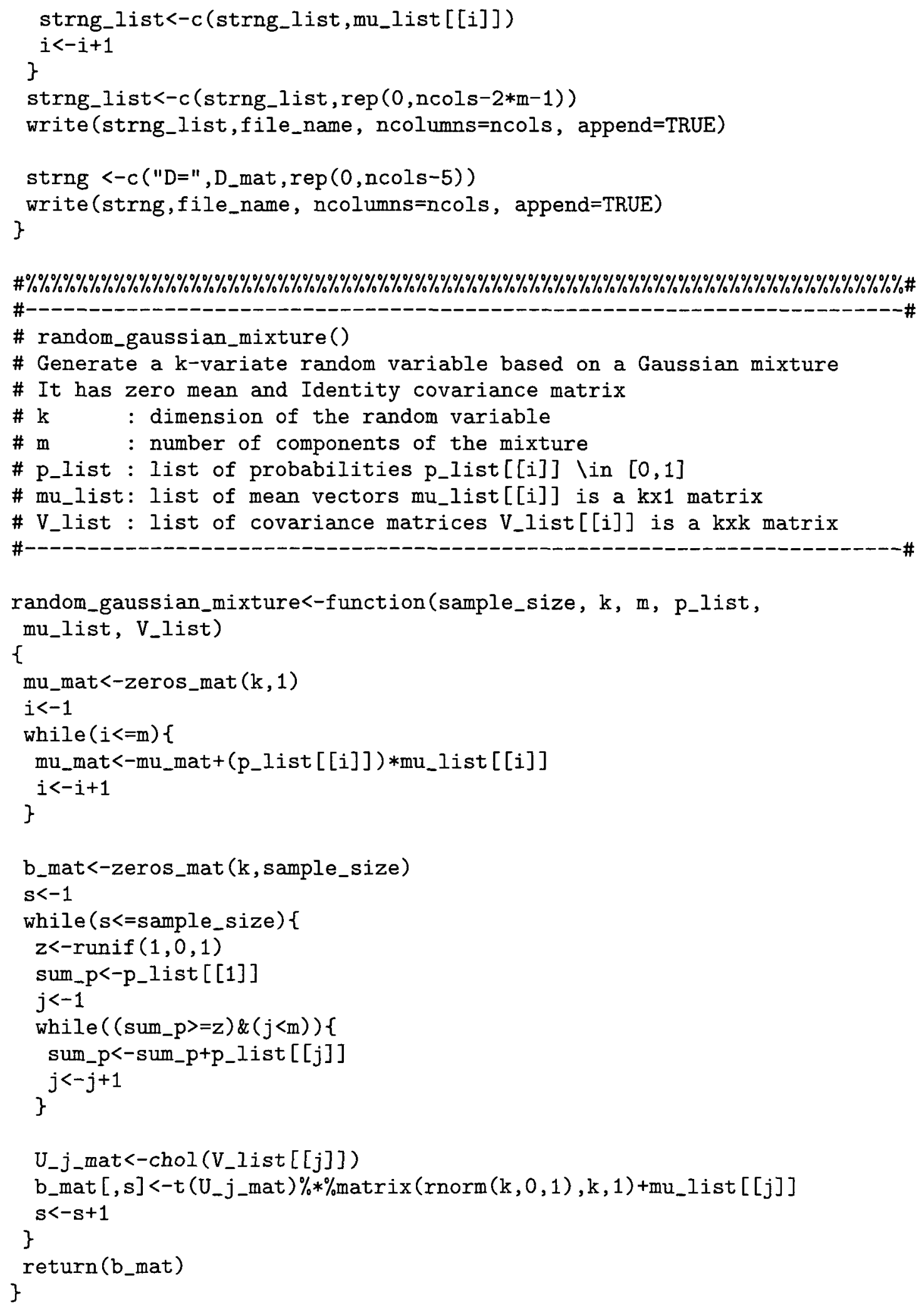




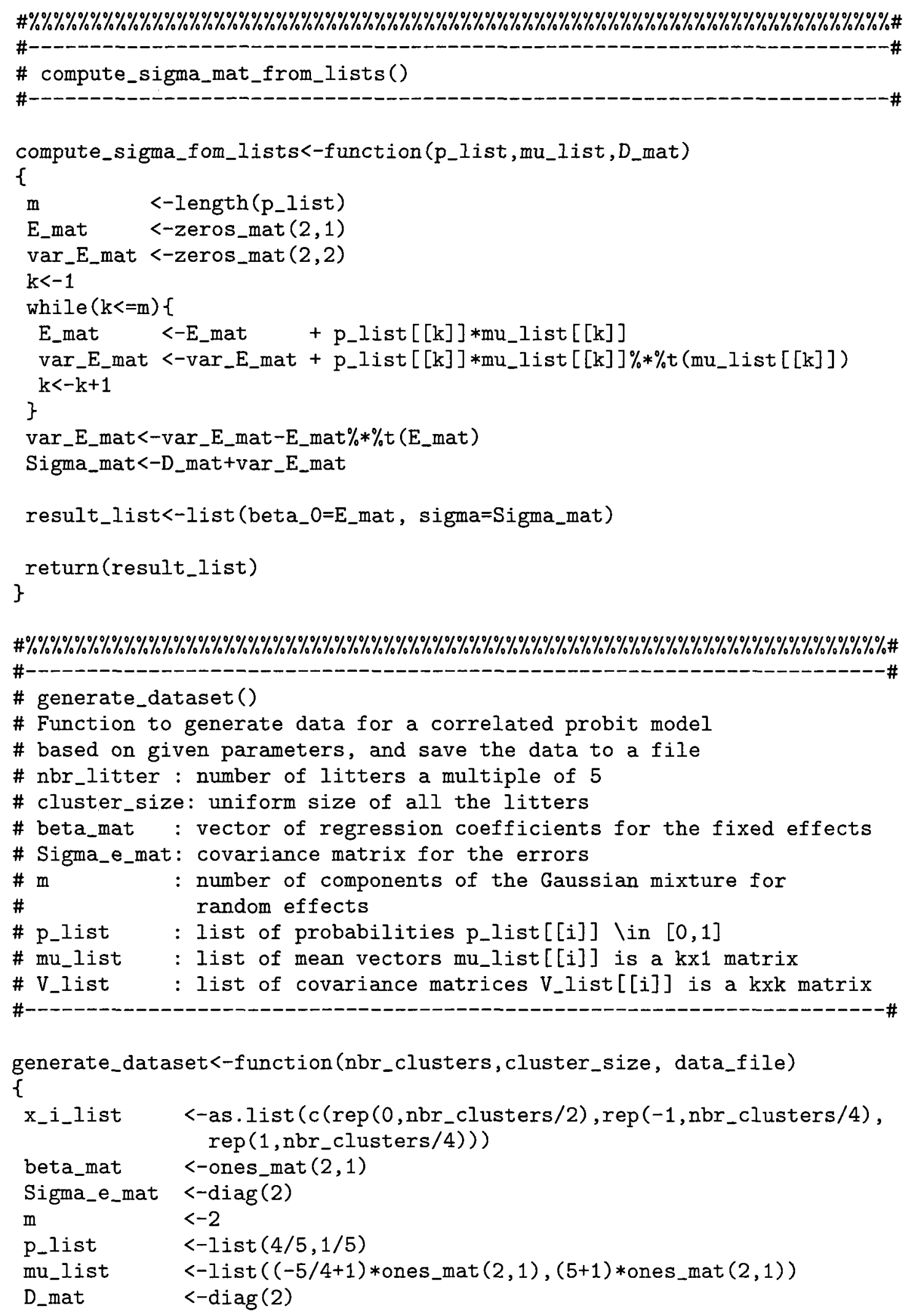




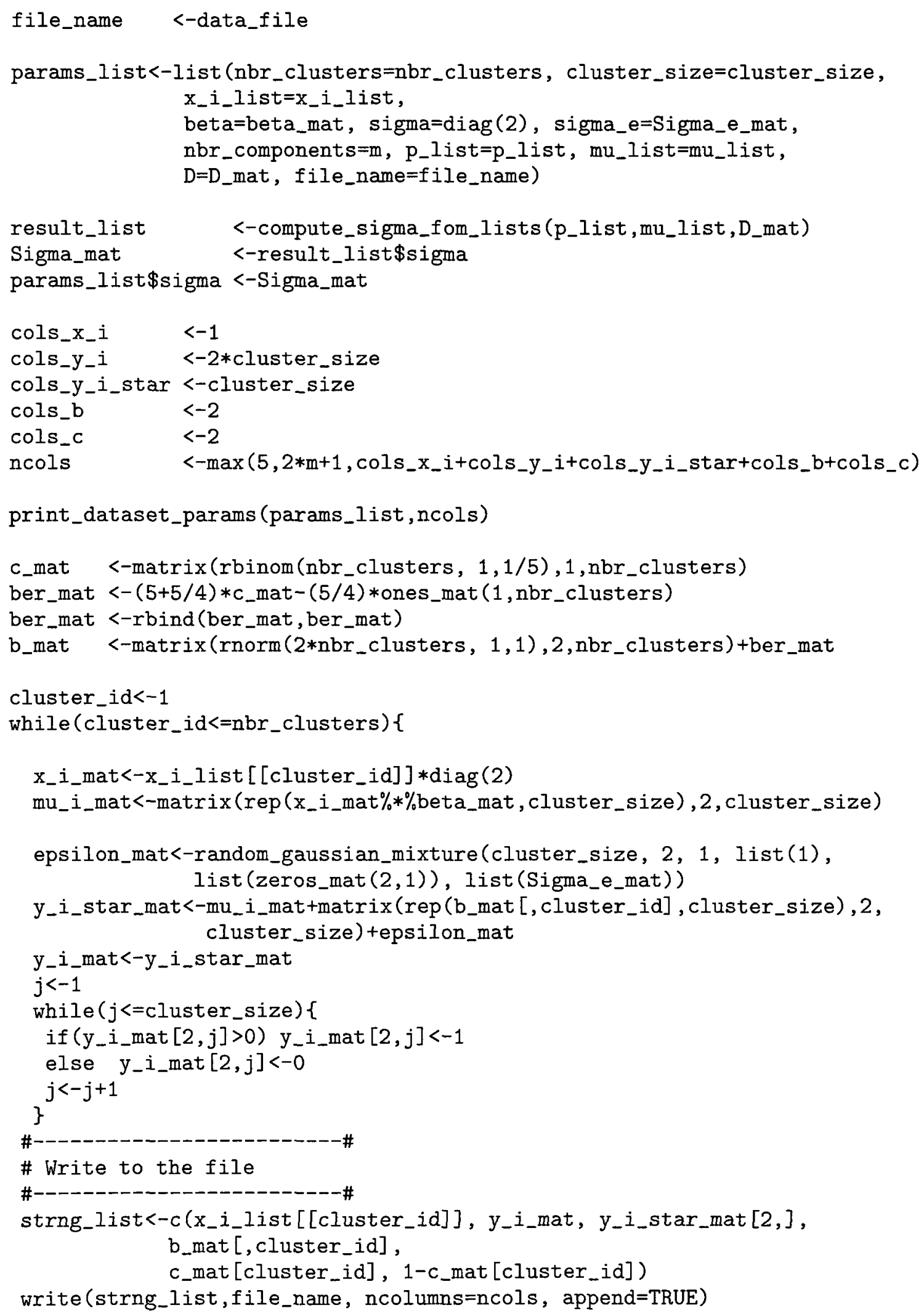




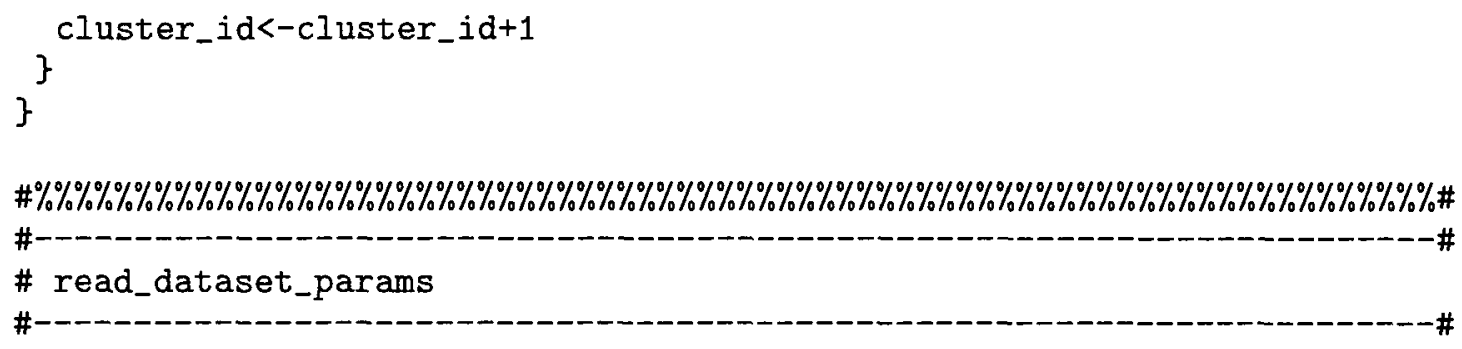

read_dataset_params<-function(file_name)

\{

file_data<-read.table (file_name, header=FALSE)

ncols<-dim(file_data $[1]),[2]$

nbr_clusters<-as.double (file_data $[1],[2]$ )

cluster_size<-as.double(file_data[2,] [2])

beta_mat<-matrix (as.double (file_data $[3],[2: 3]), 2,1$ )

Sigma_mat<-matrix(as.double(file_data $[4],[2: 5]), 2,2)$

Sigma_e_mat<-matrix (as.double (file_data $[5],[2: 5]), 2,2$ )

$m<-a s . i n t e g e r\left(f i l e \_d a t a[6],[2]\right)$

p_list<-list()

$i<-1$

while $(i<=m)\{$

p_list $[[i]]<-a s . d o u b l e\left(f i l e \_d a t a[7],[i+1]\right)$

$i<-i+1$

\}

mu_list $<-$ list ()

$i<-1$

while $(i<=m)\{$

mu_list $[[i]]<-\operatorname{matrix}($ as.double (file_data $[8],[(2 * i):(2 * i+1)]), 2,1)$ $i<-i+1$

\}

D_mat <-matrix(as.double (file_data $[9],[(2+4 *(1-1)):(1+4 * 1)]), 2,2)$

params_list <-list (nbr_clusters=nbr_clusters, cluster_size=cluster_size, beta=beta_mat, sigma $=$ Sigma_mat, sigma_e=Sigma_e_mat, nbr_components $=m$, p_list=p_list, mu_list=mu_list, $\mathrm{D}=\mathrm{D} \_$mat)

return(params_list)

\} 


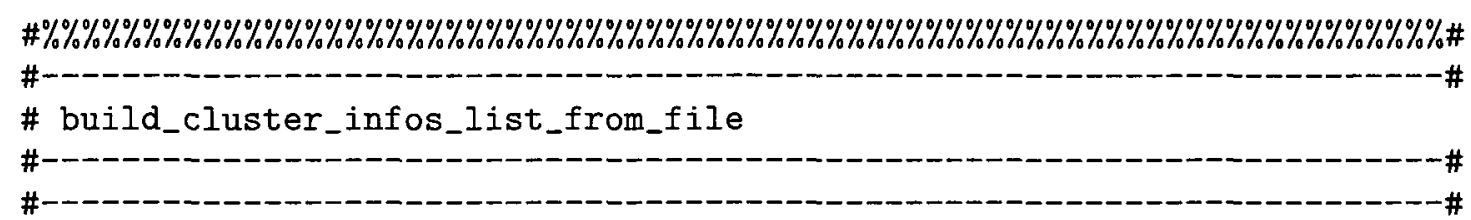

build_cluster_infos_list_from_file<-function(file_name)

\{

file_data <-read.table(file_name, header=FALSE, as.is=1)

params_list <-read_dataset_params (file_name)

m <-params_list\$nbr_components

cluster_size <-params_list\$cluster_size

nbr_clusters <-params_list\$nbr_clusters

total_obs <-nbr_clusters*cluster_size

id_list <-as.list(c(1:nbr_clusters))

$\mathrm{x}_{-} i_{-}$list $<-l i s t()$

size_list <-as.list(rep(cluster_size, nbr_clusters))

$\begin{array}{ll}\text { y_i_list } & <- \text { list }() \\ \text { y_i_star_list } & <- \text { list }() \\ \text { b_i_list } & <-l i s t() \\ \text { c_i_list } & <-l i s t()\end{array}$

s1_list<-list()

s2_list<-list ()

s3_list<-list()

s4_list<-list ()

s5_list<-list ()

s6_list<-list()

s7_list<-list ()

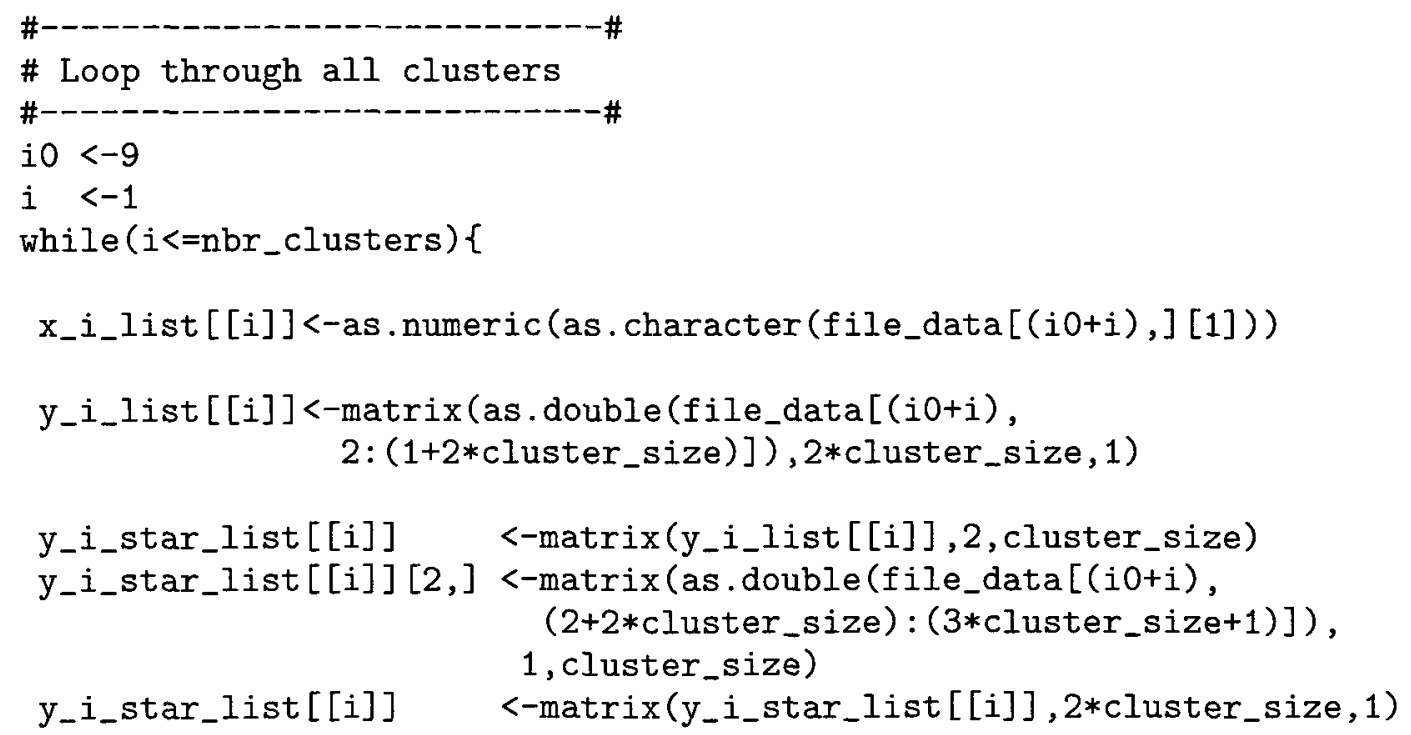




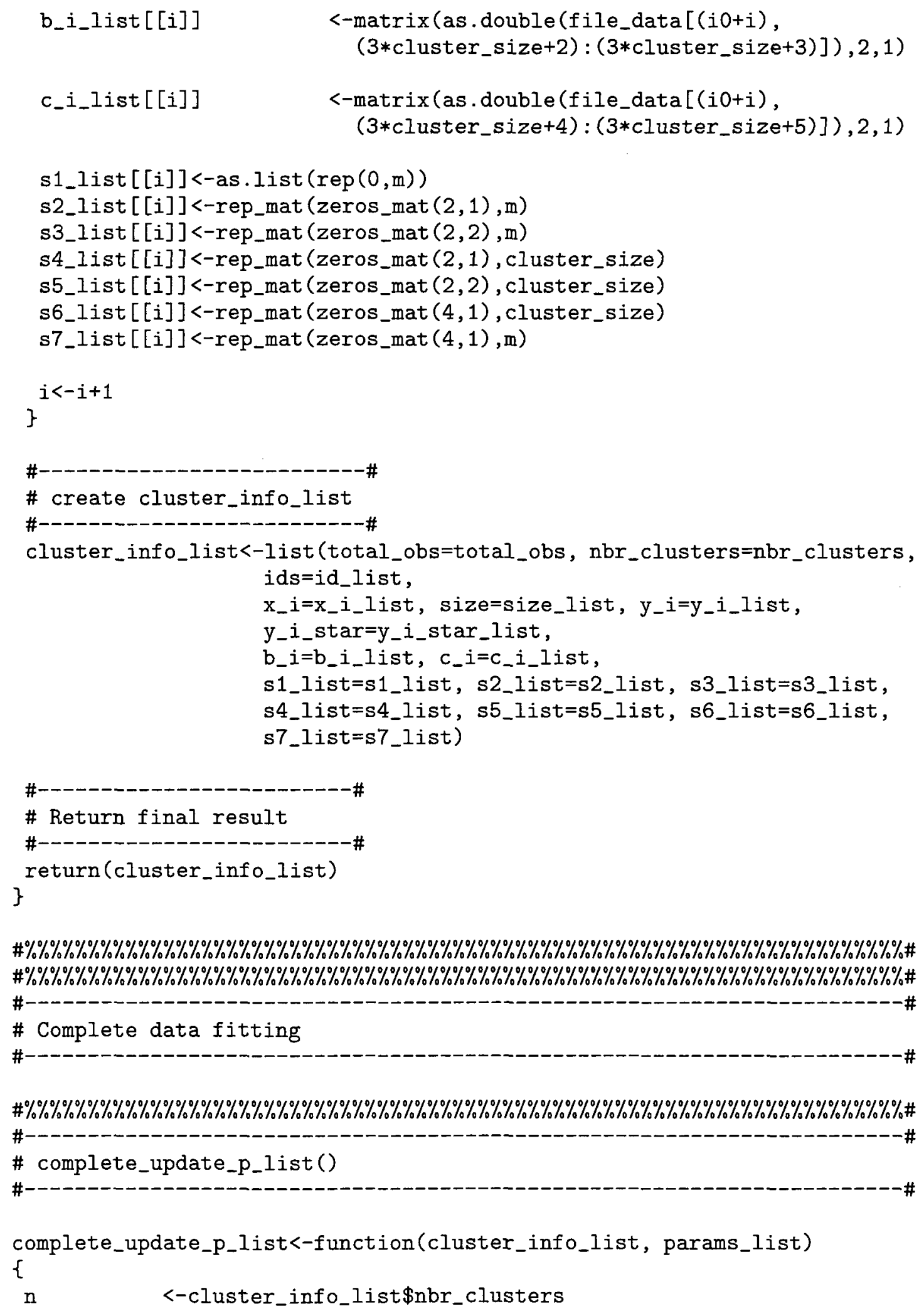




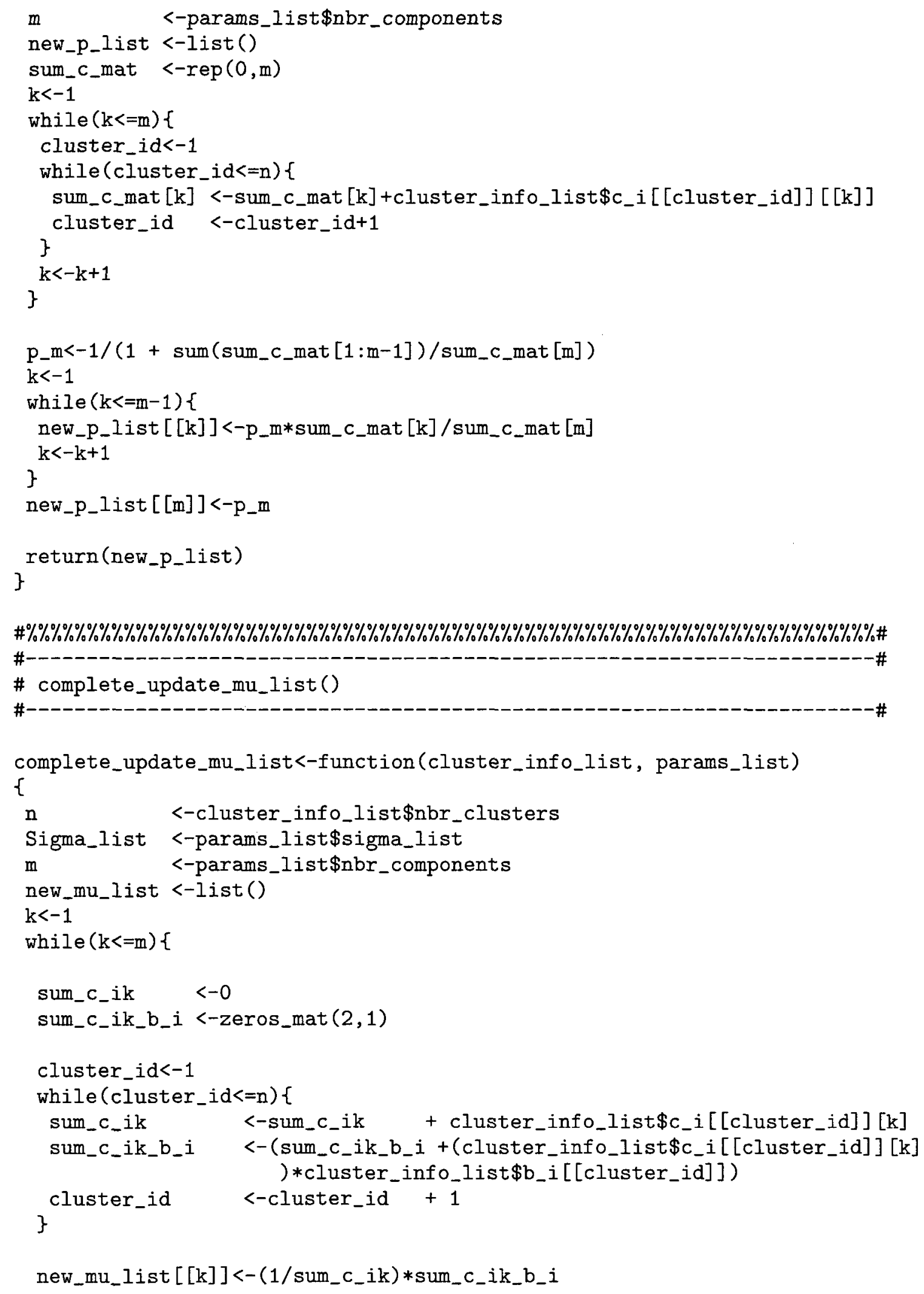




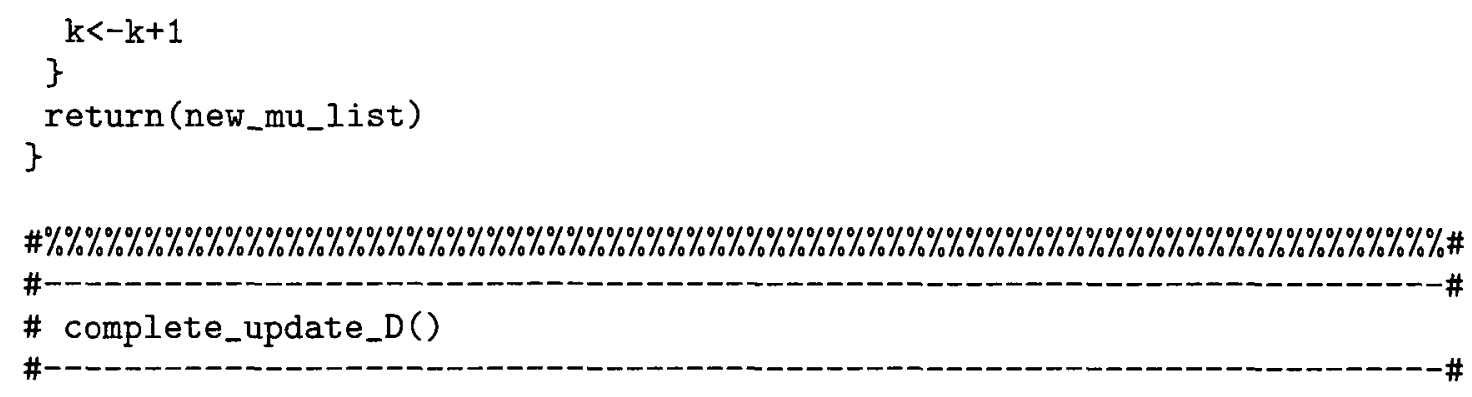

complete_update_D<-function(cluster_info_list, params_list)

\{

n <-cluster_info_list\$nbr_clusters

m <-params_list\$nbr_components

mu_list <-params_list $\$ m u_{-}$list

new_D <-zeros_mat $(2,2)$

total_c_ik $<-0$

$\mathrm{k}<-1$

while $(k<=m)\{$

mu_k_mat<-mu_list $[[\mathrm{k}]]$

sum_c_ik $\quad<-0$

sum_c_ik_b_i <-zeros_mat $(2,1)$

sum_c_ik_b_i_t_b_i <-zeros_mat $(2,2)$

cluster_id<-1

while (cluster_id $<=n$ ) \{

$c_{-} i k \quad<-c l u s t e r_{-}$info_list $\$ c_{-} i[[$ cluster_id] $][[\mathrm{k}]]$

sum_c_ik <-sum_c_ik $+c_{-} i k$

sum $c_{-} i k_{-} b_{-} i \quad<-\left(s_{-} m_{-} c_{-} i k_{-} b_{-} i+\right.$

$c_{-} i k * c l u s t e r_{-} i n f o_{-} l i s t \$ b_{-} i[[$ cluster_id] $])$

sum_c_ik_b_i_t_b_i <-sum_c_ik_b_i_t_b_i + c_ik*cluster_info_list\$b_i[[cluster_id] $] \% * \%$ t

cluster_info_list\$b_i[[cluster_id] $]$ ))

\}

cluster_id <-cluster_id+1

total_c_ik <-total_c_ik + sum_c_ik

new_ $D<-n e w_{-} D+s u m_{-} c_{-} i k_{-} b_{-} i_{-} t_{-} b_{-} i+s u m_{-} c_{-} i k * m u_{-} k_{-} m a t \% * \%$ (mu_k_mat) -(

$k<-k+1$ sum_c_ik_b_i\%*\%t(mu_k_mat) + mu_k_mat $\left.\% * \% t\left(s u m_{-} c_{-} i k_{-} b_{-} i\right)\right)$

\}

new_D $<-(1 /$ total_c_ik) $*$ new_D

return(new_D)

\} 


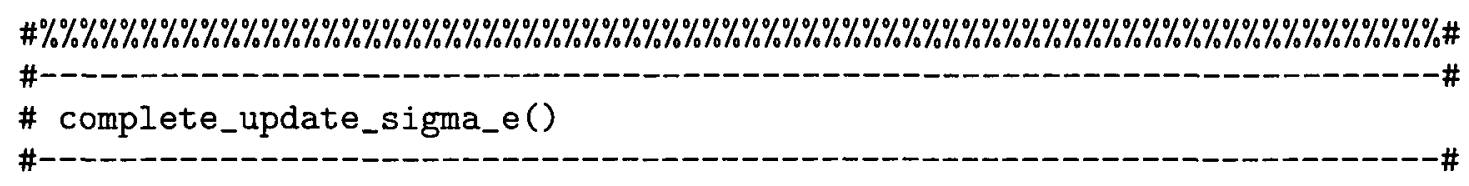

complete_update_sigma_e<-function(cluster_info_list, params_list)

\{

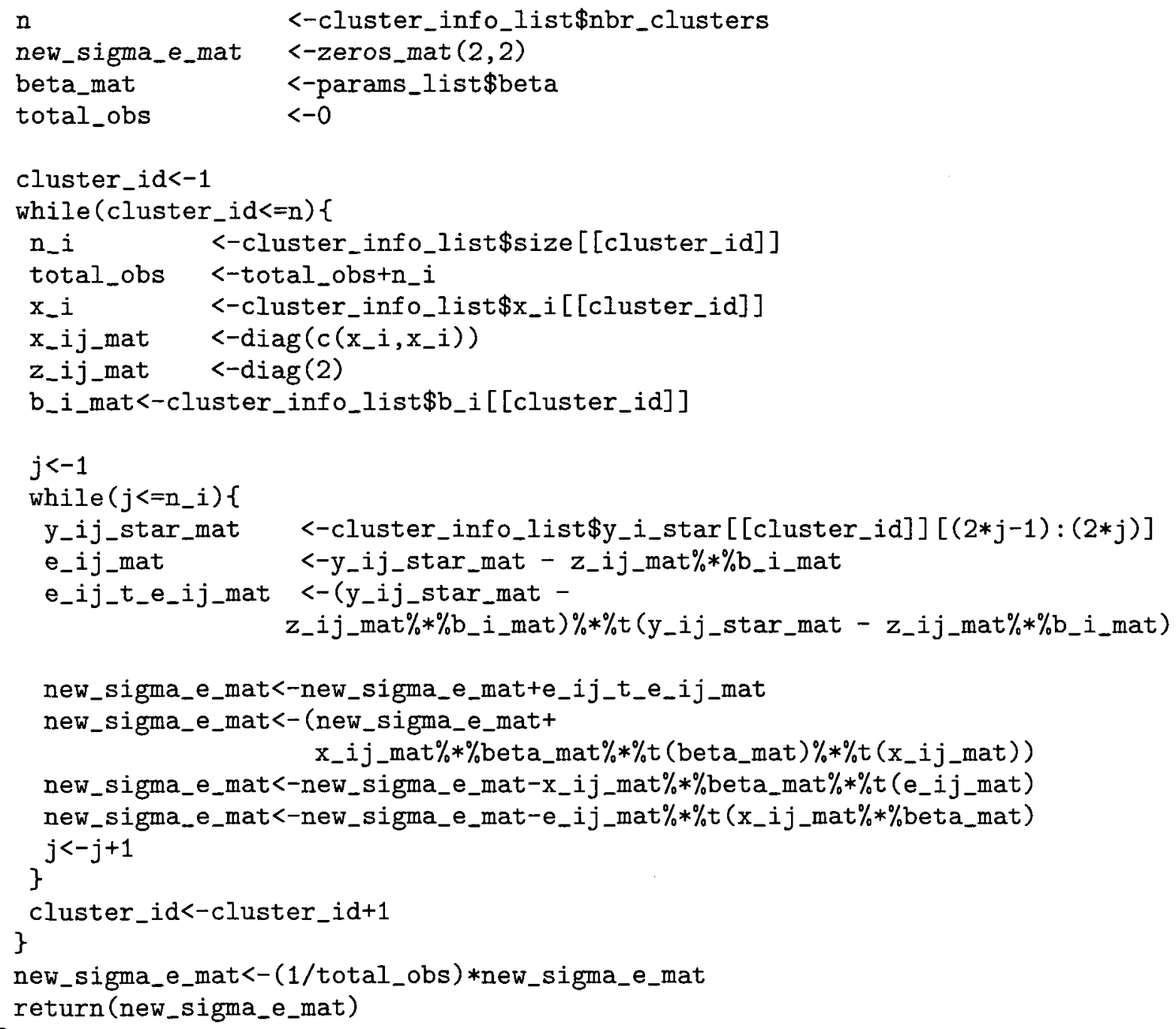




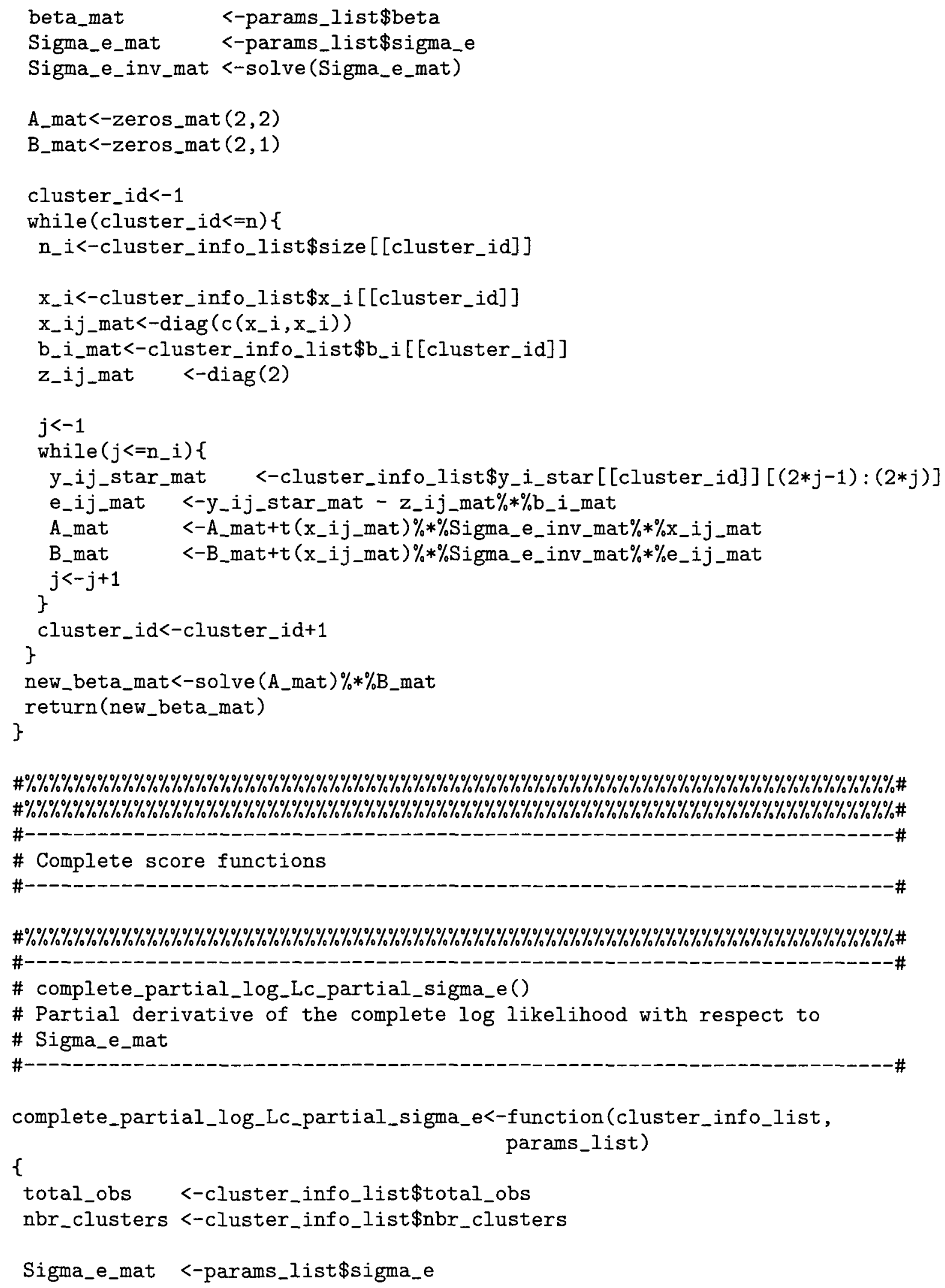




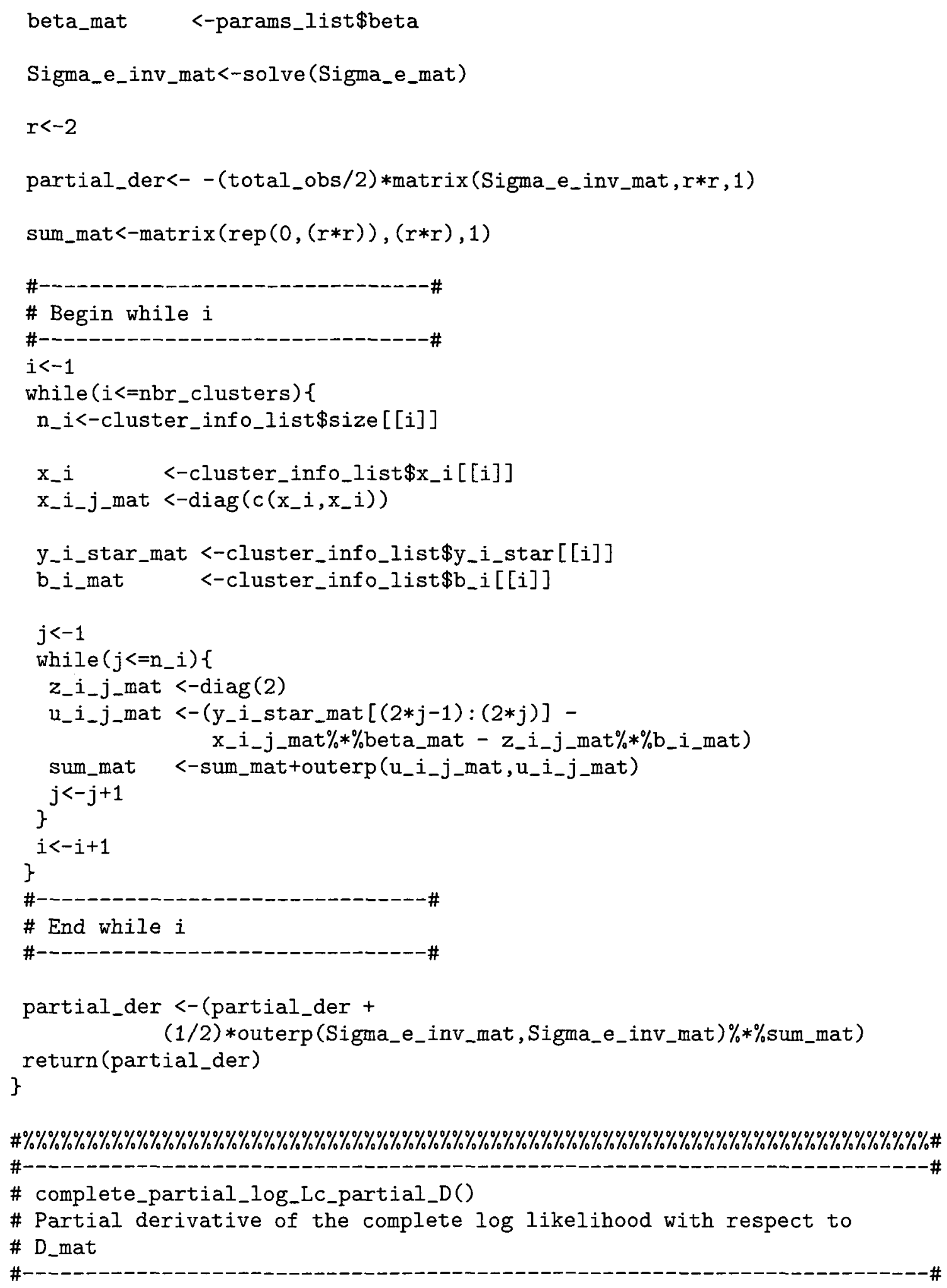




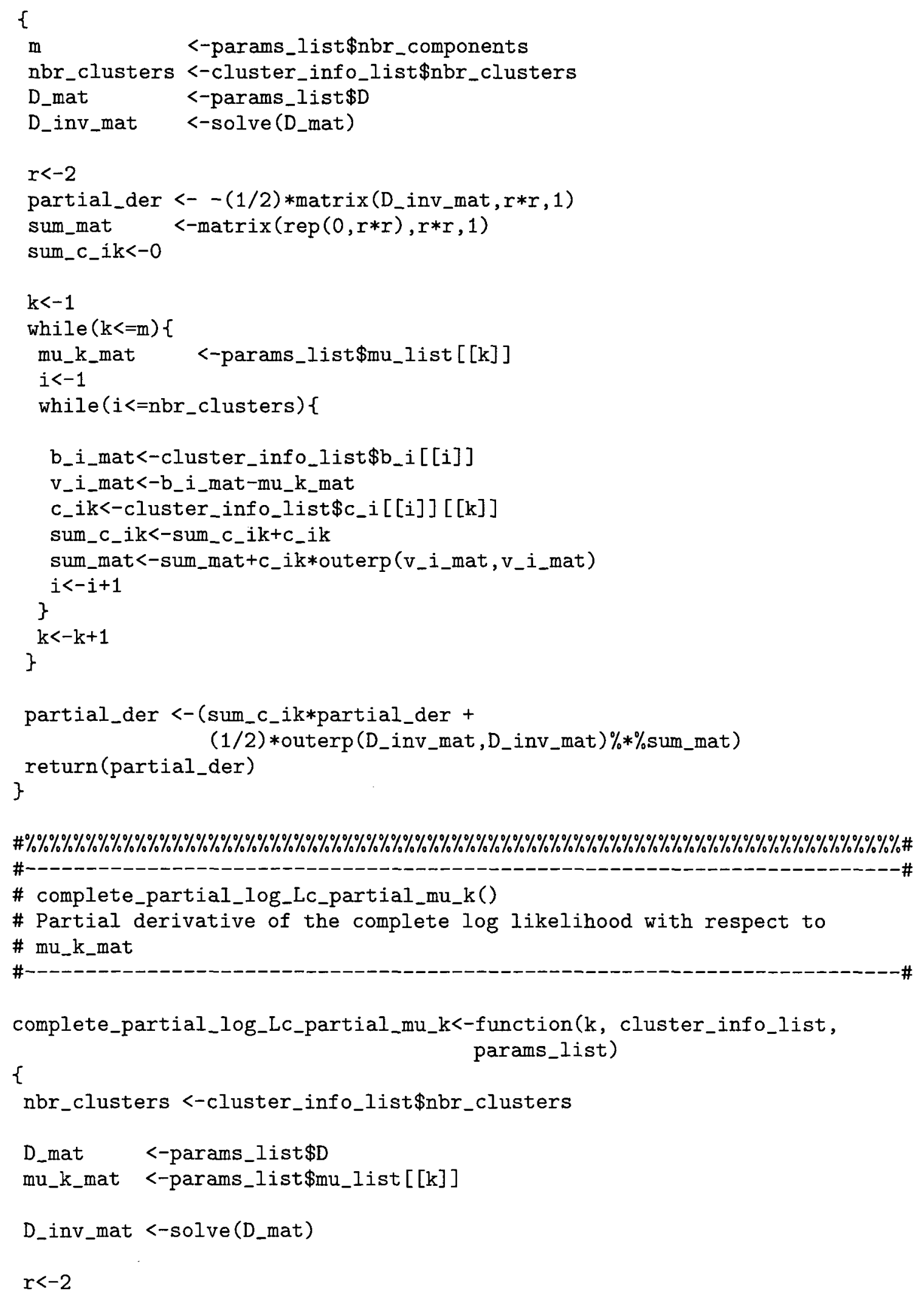




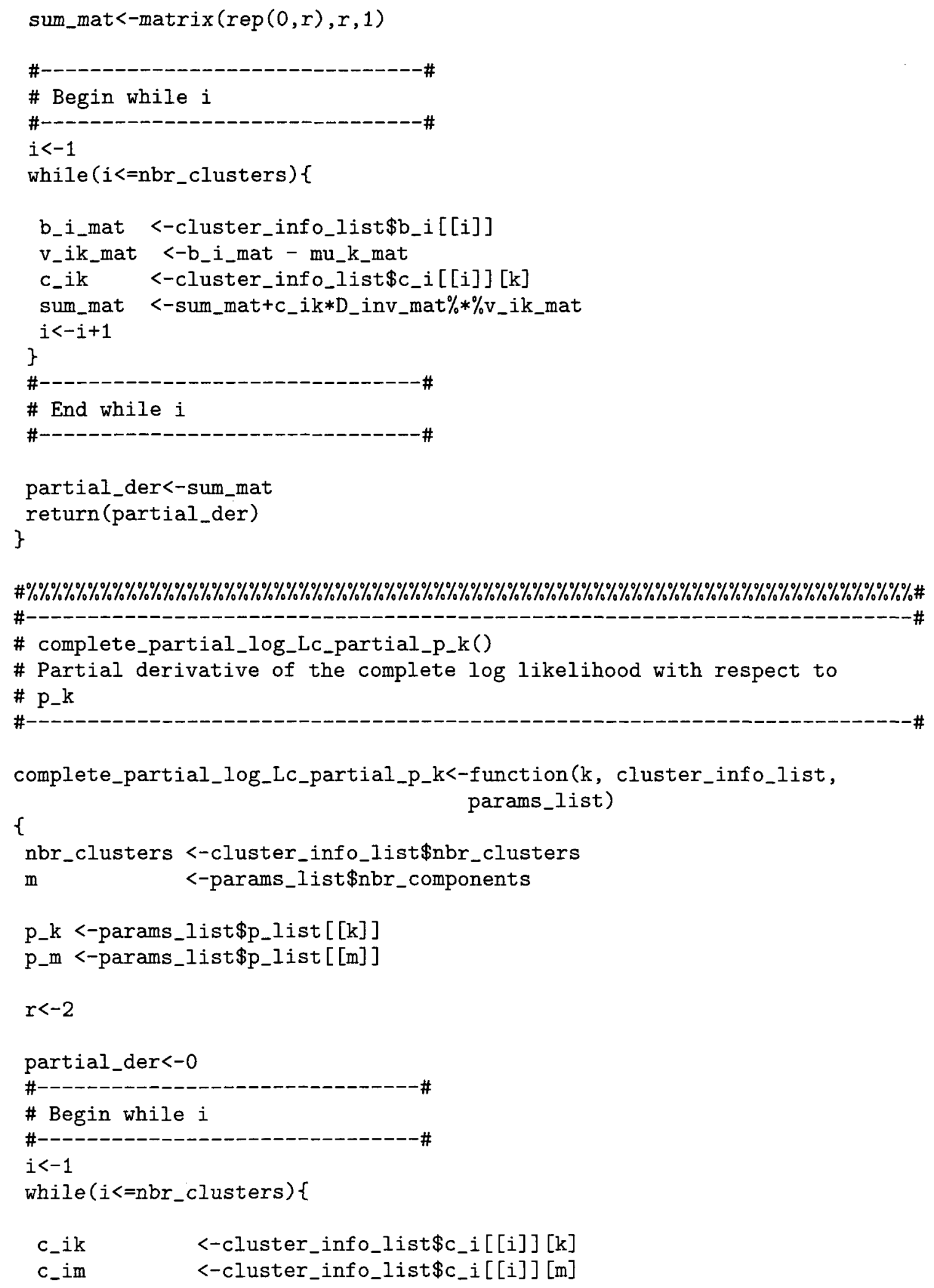



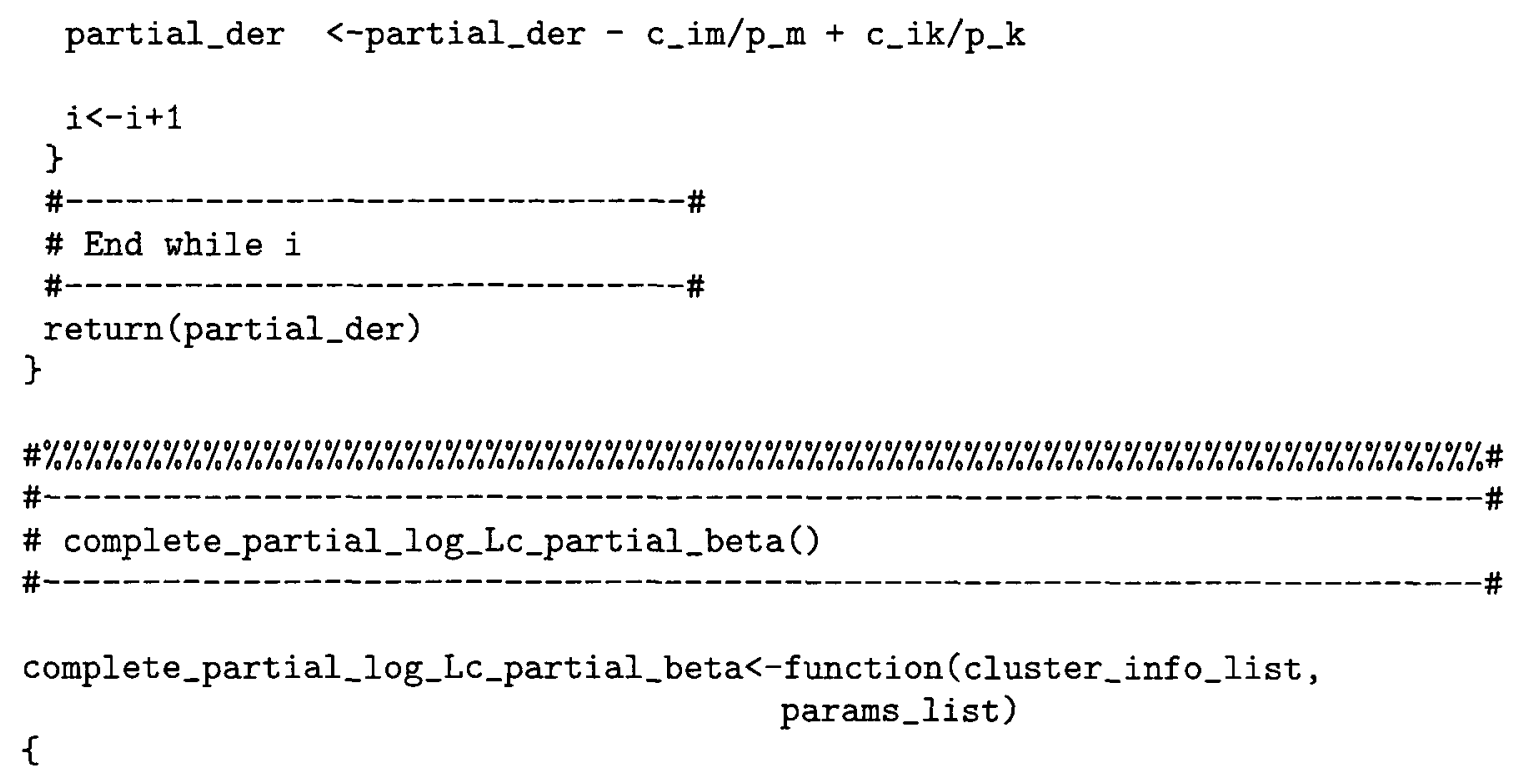


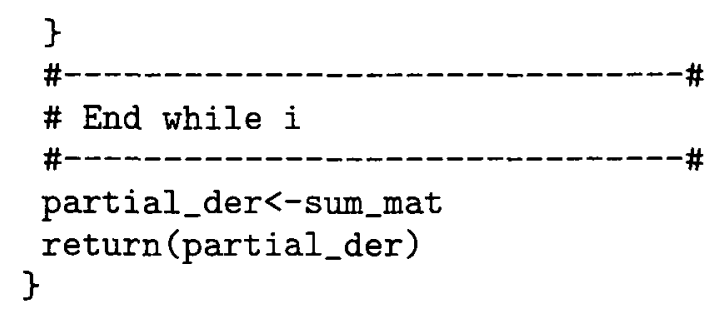

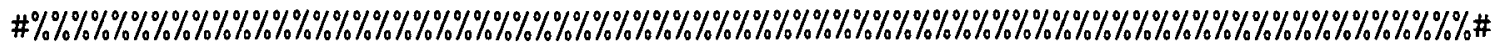
\#\% \% \% \% \% \% \% \% \% \% \% \% \% \% \% \% \% \% \% \% \% \% \% \% \% \% \% \% \% \% \% \% \% \% \% \% \% \% \% \% \% \% \% \% \% \% \% \% \% \% \% \% \% \% \% \% \% \% \% \% \% \% \% \% \% \% \% \% \% \% \% \% \# 


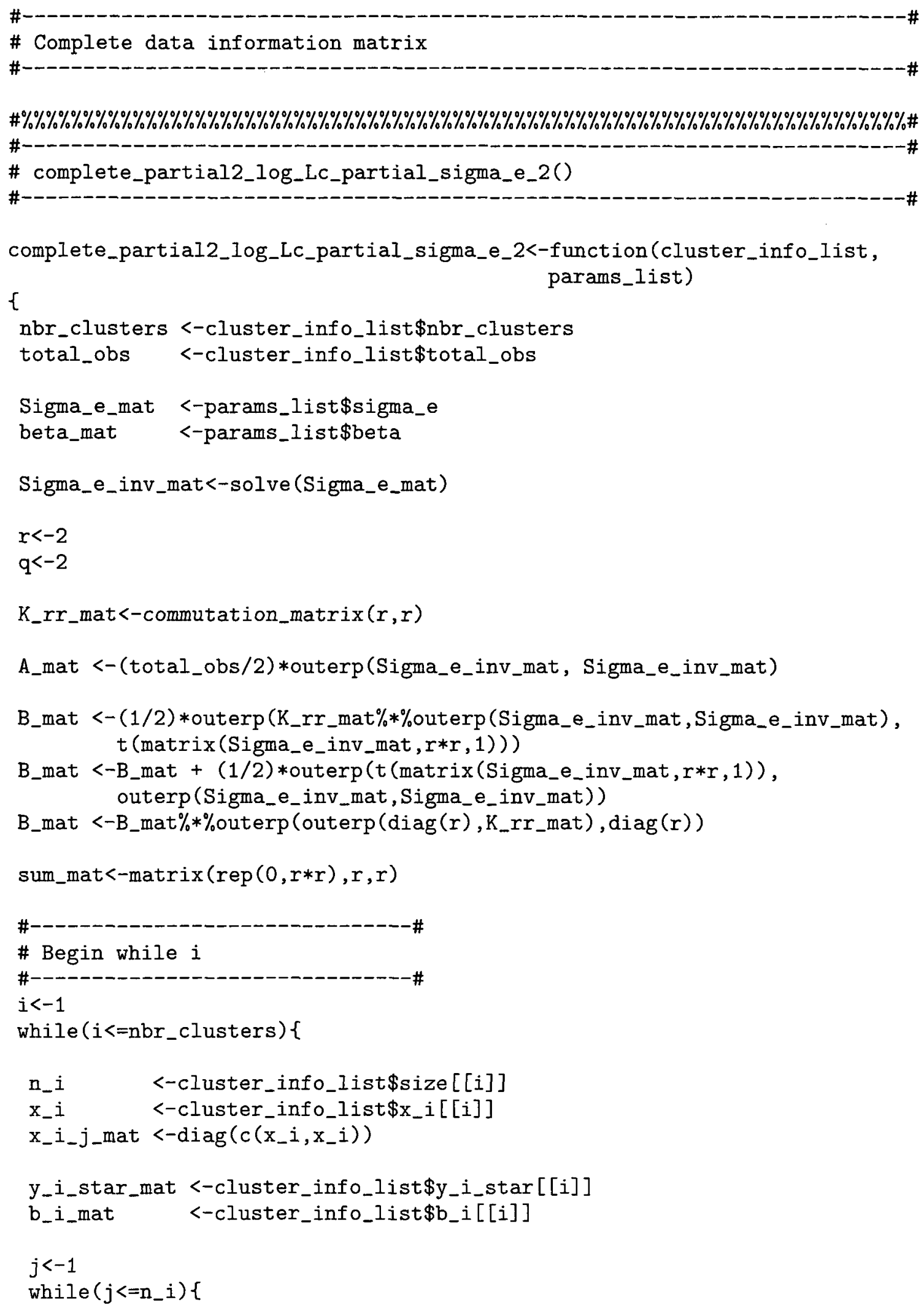




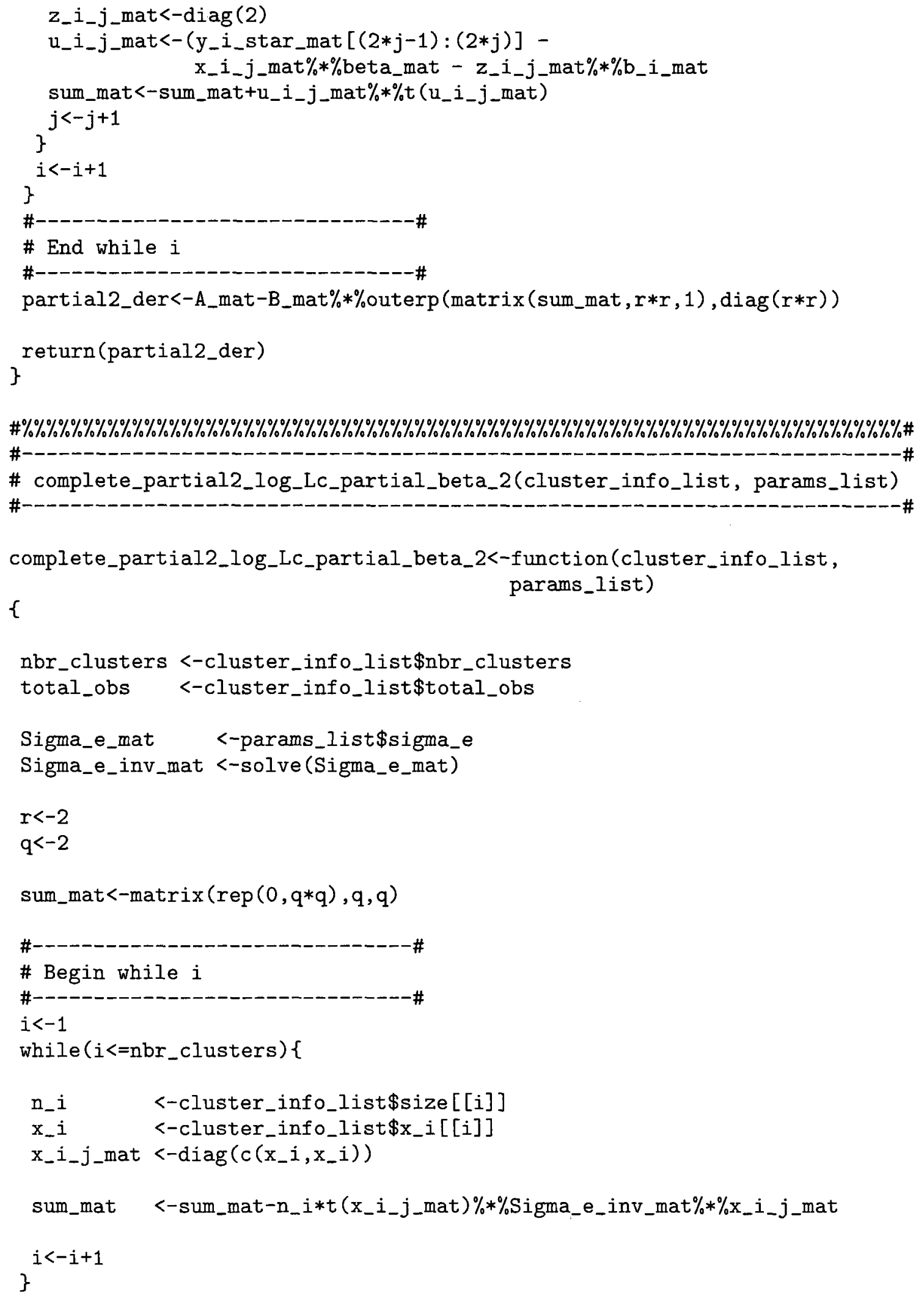




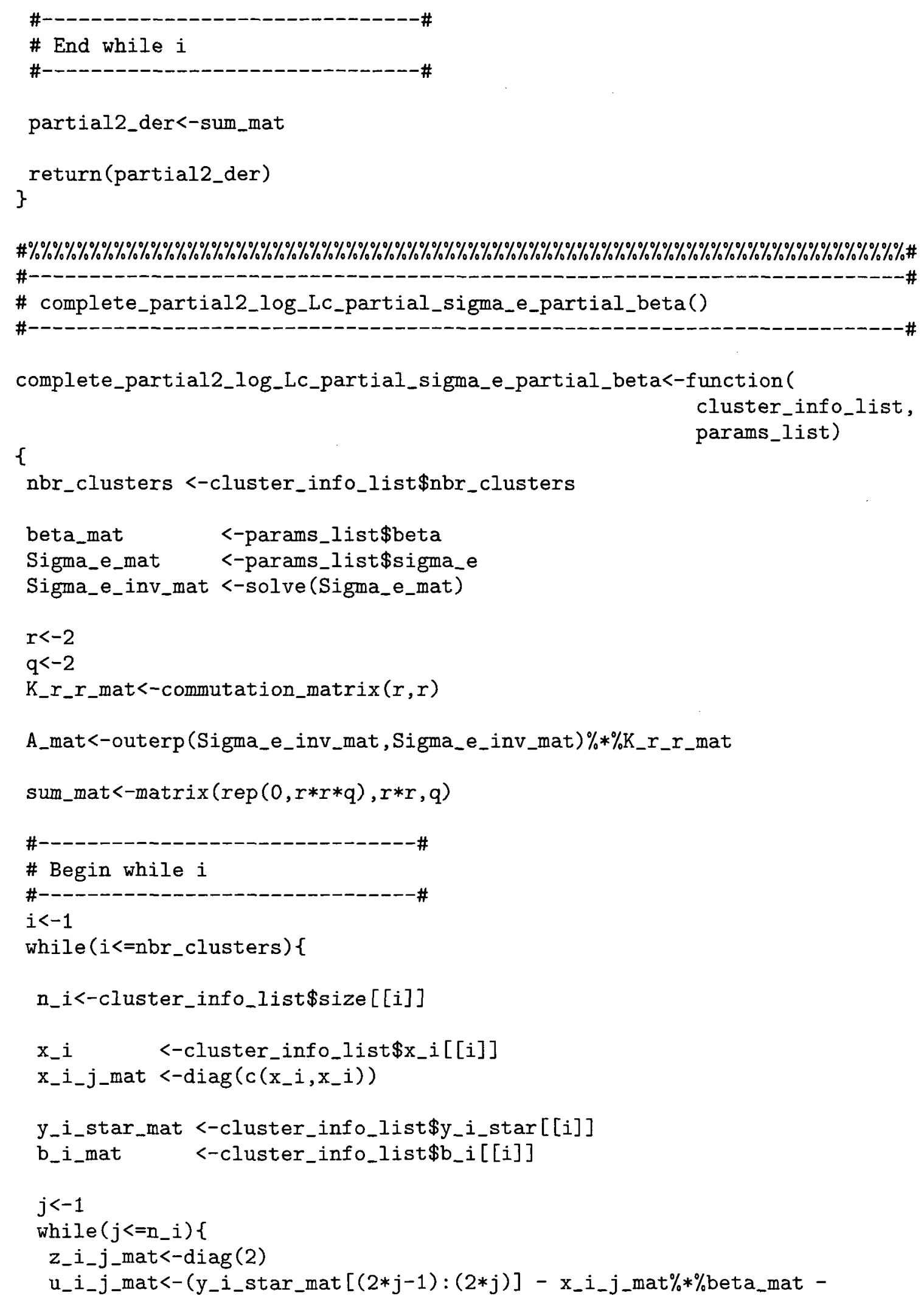




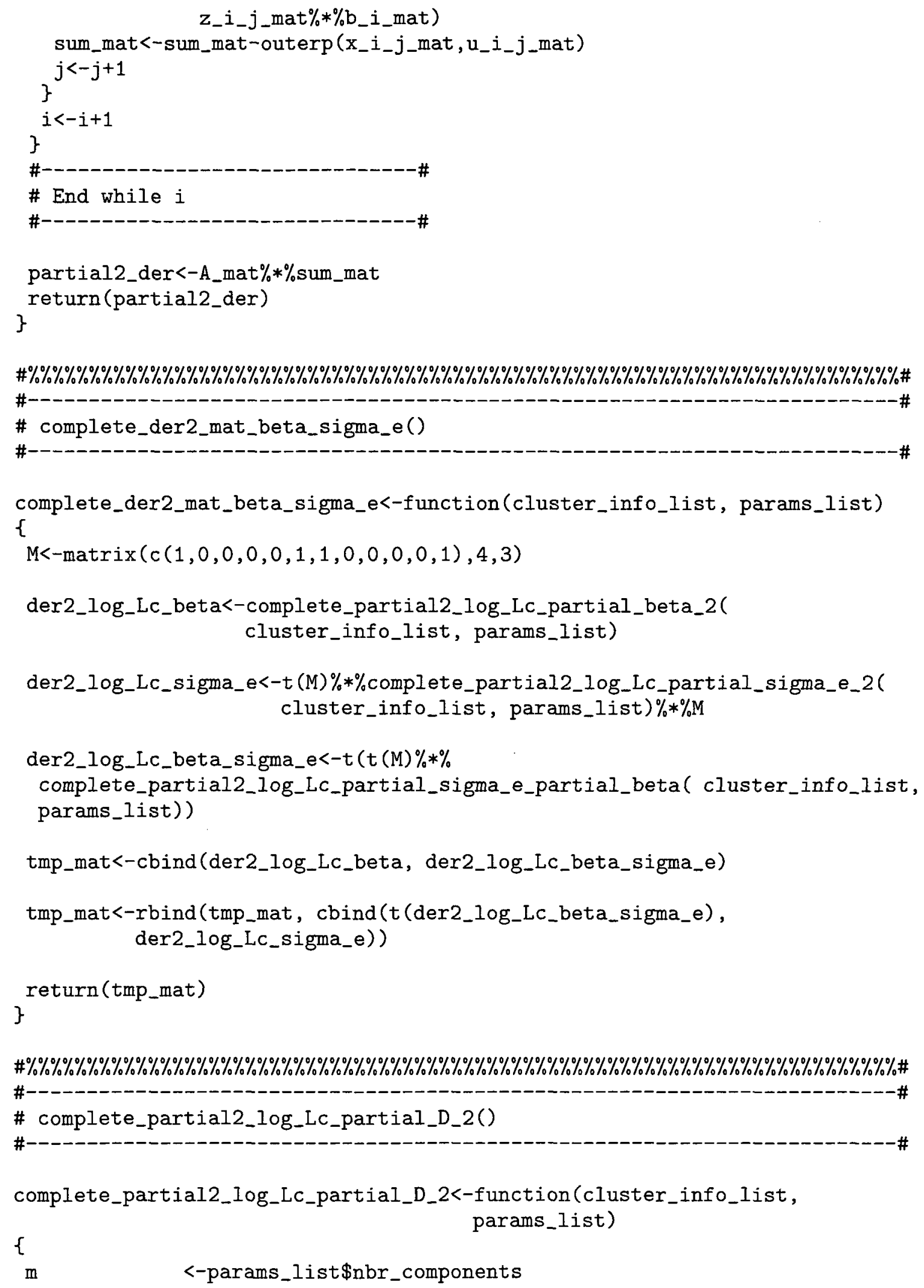




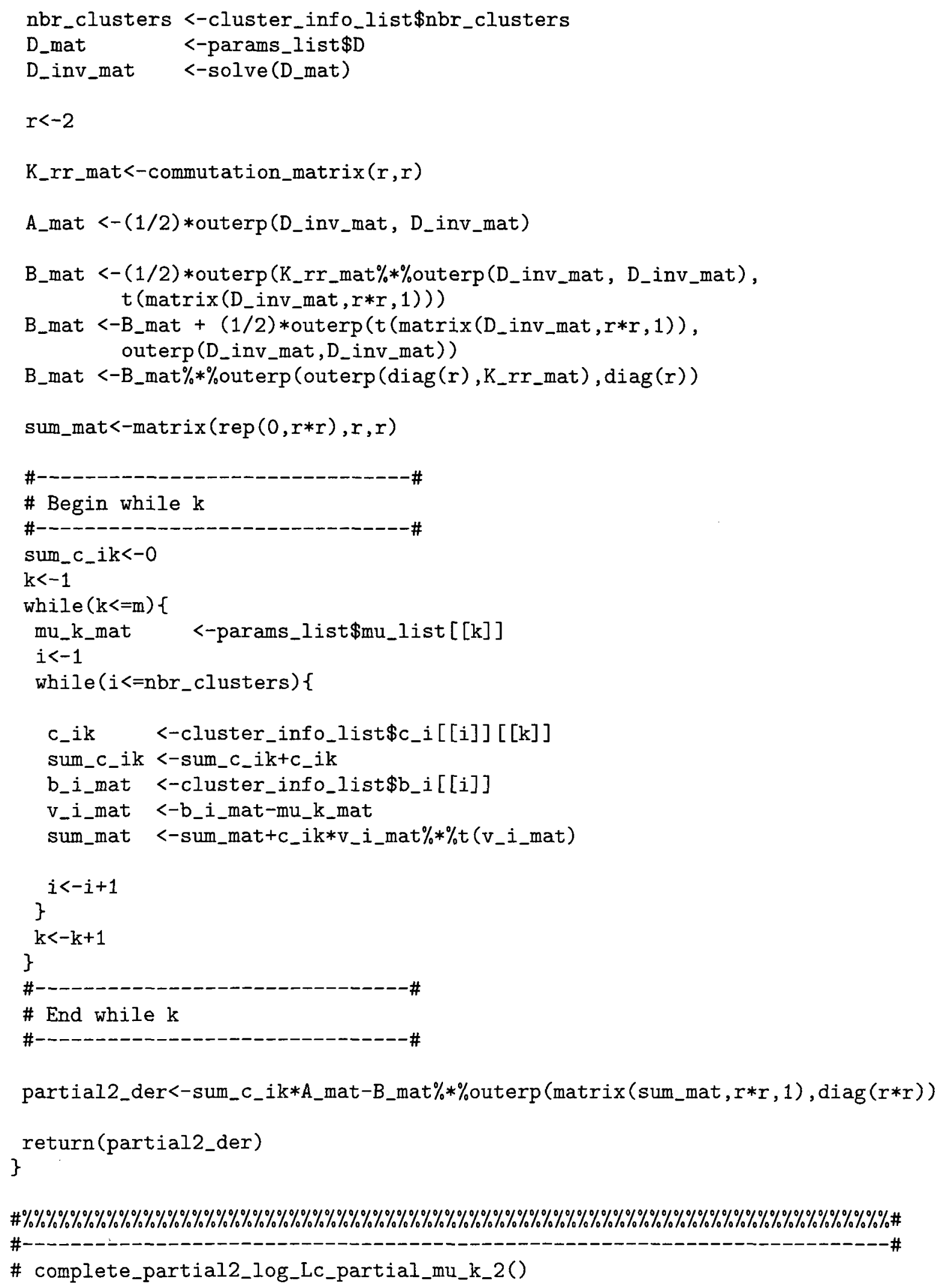




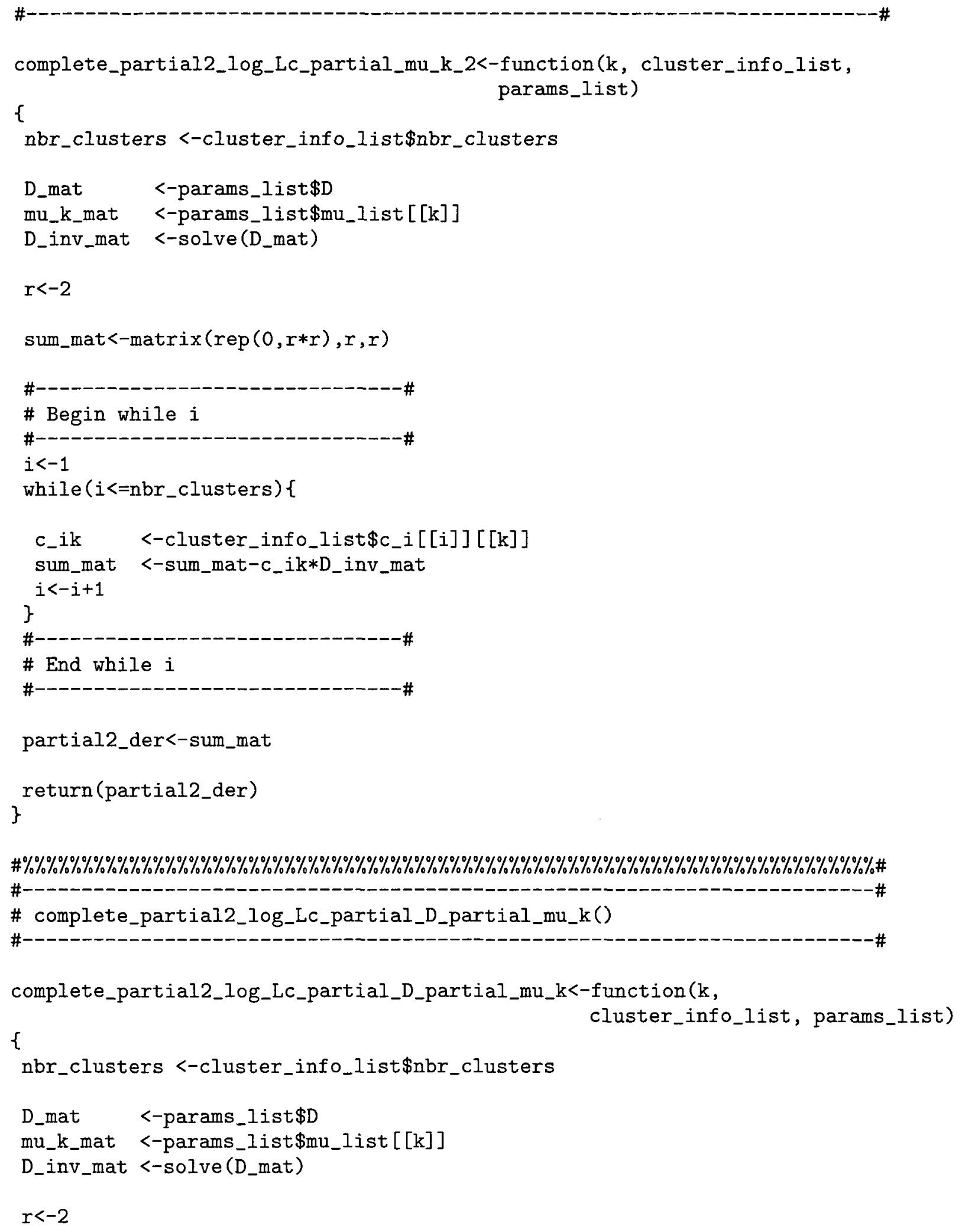




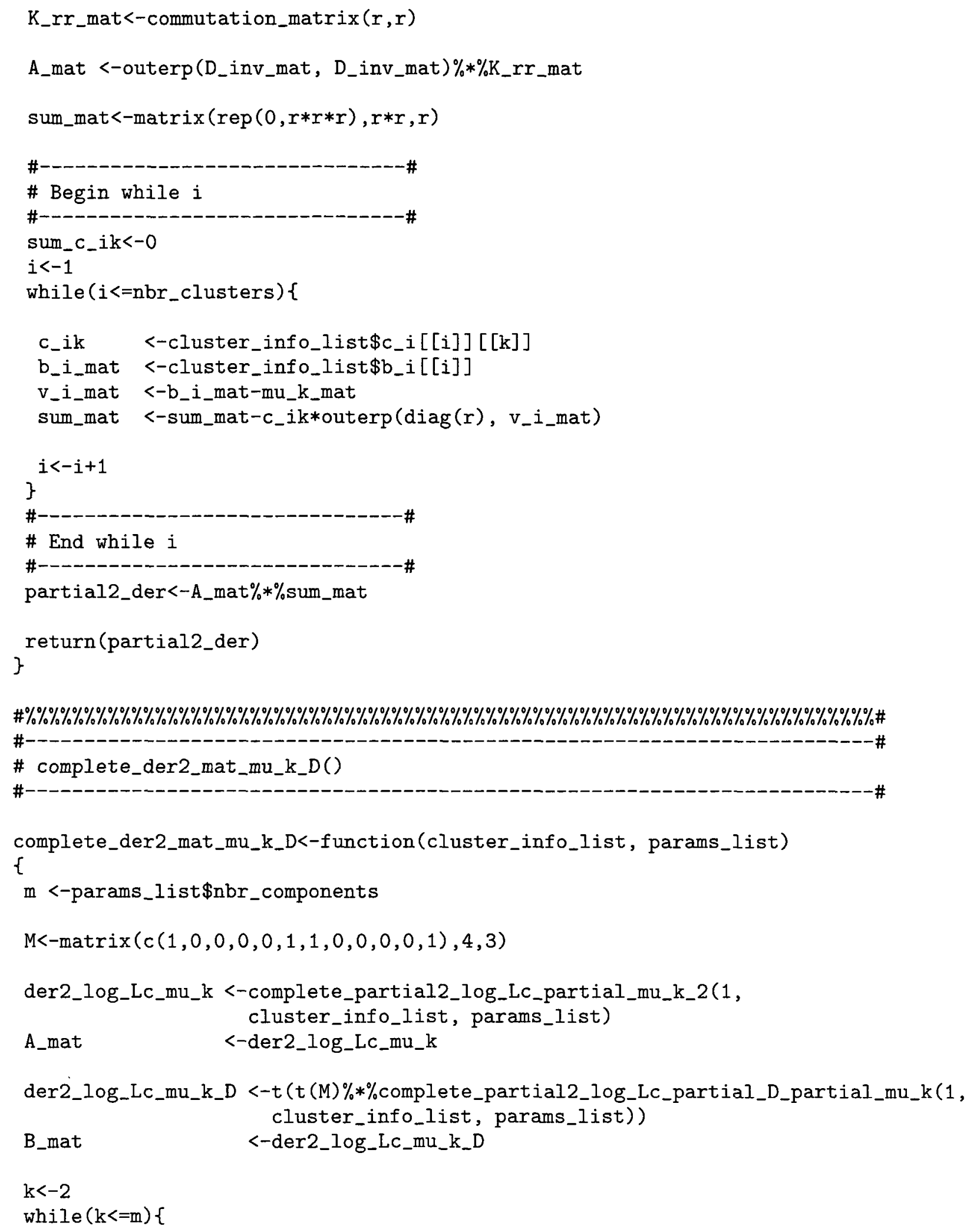




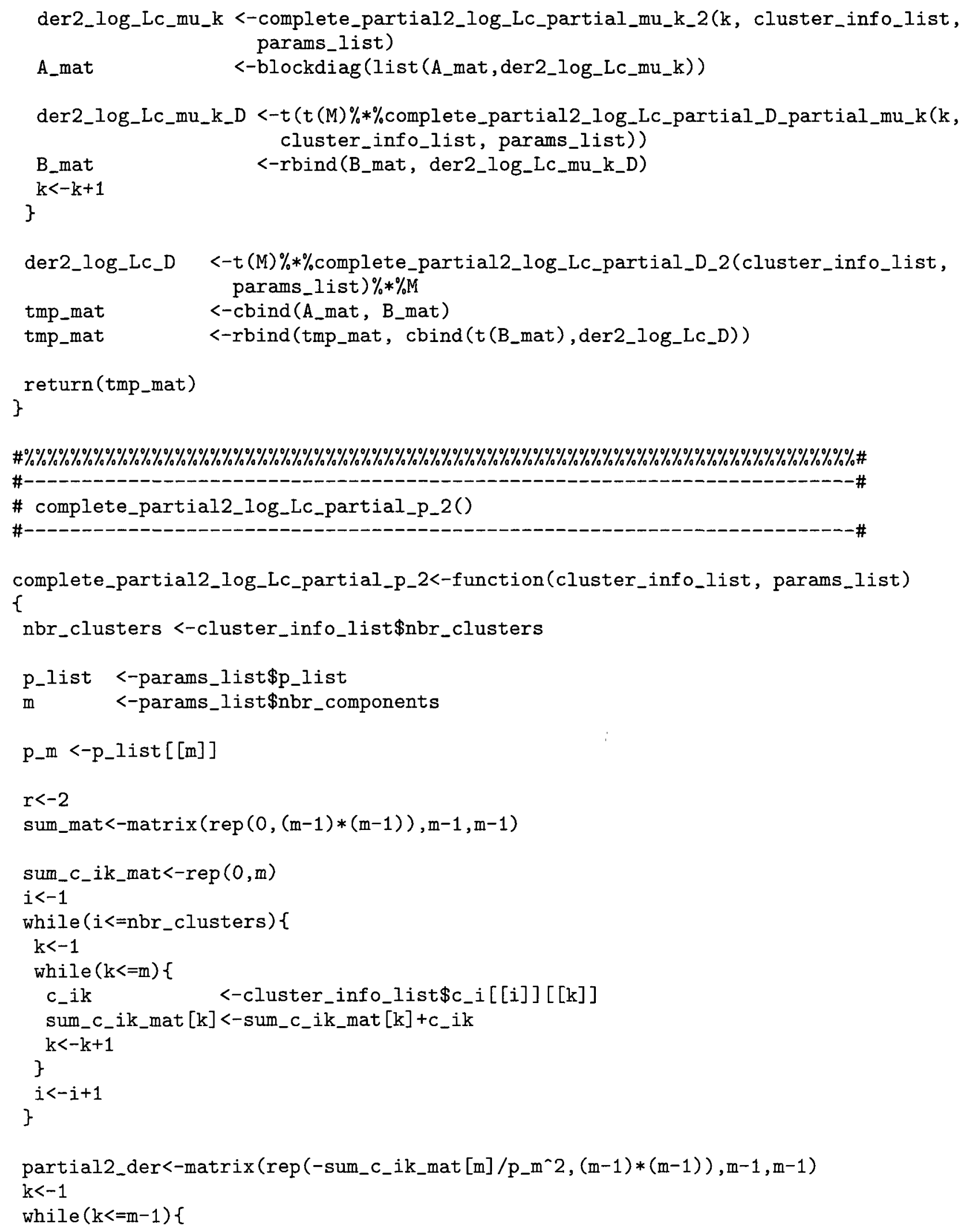




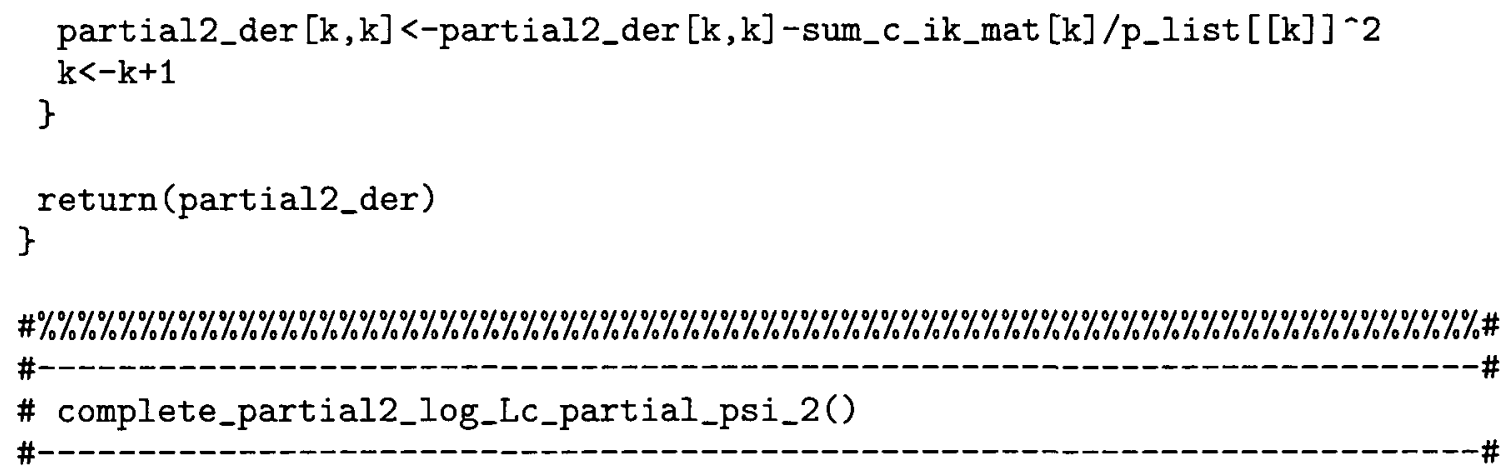




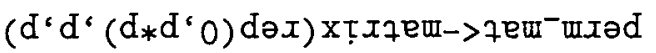

$$
\begin{aligned}
& \}(I<Y) F ?
\end{aligned}
$$

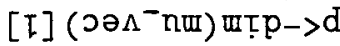

\} (7еس'

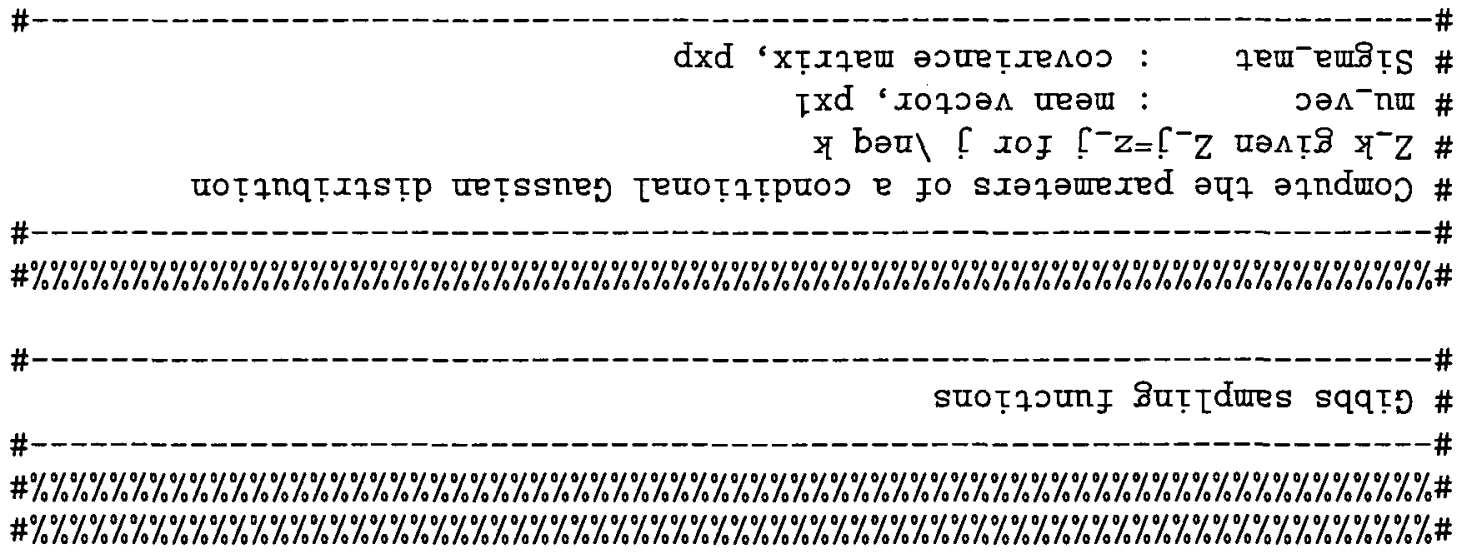

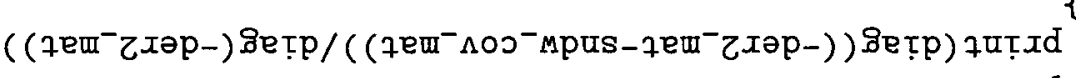

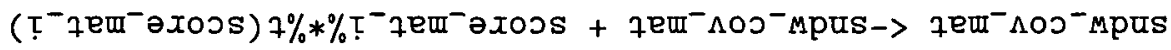

(7stit'surexed '7stT'ofut'xә7snto

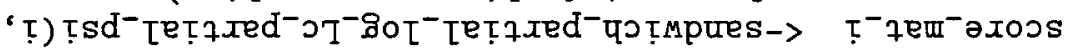

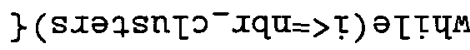

$Z->$ !

( $T^{-}$7eul(7stT' surexed ' 75 t T' Ofut'xә7snto

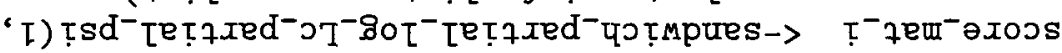

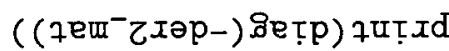
(7st? I- surexed

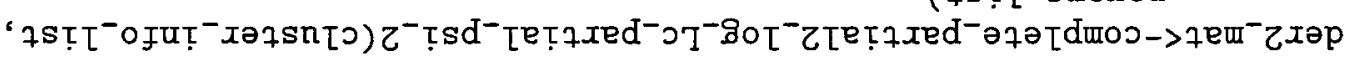

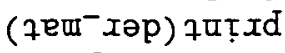
(7st? $\tau^{-}$surexed

' $72 t \tau^{-}$-

( $75 T \tau^{-}$surexed) $q$ quT $x d$

eس.

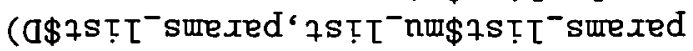

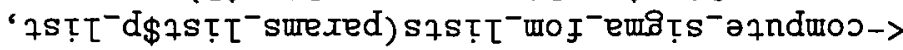

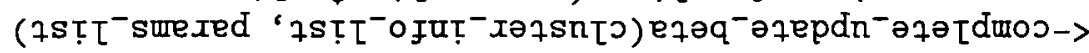

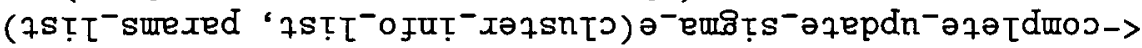

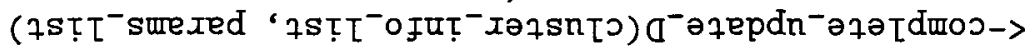

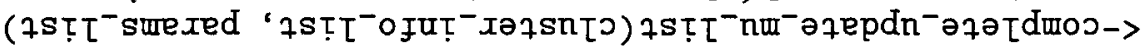




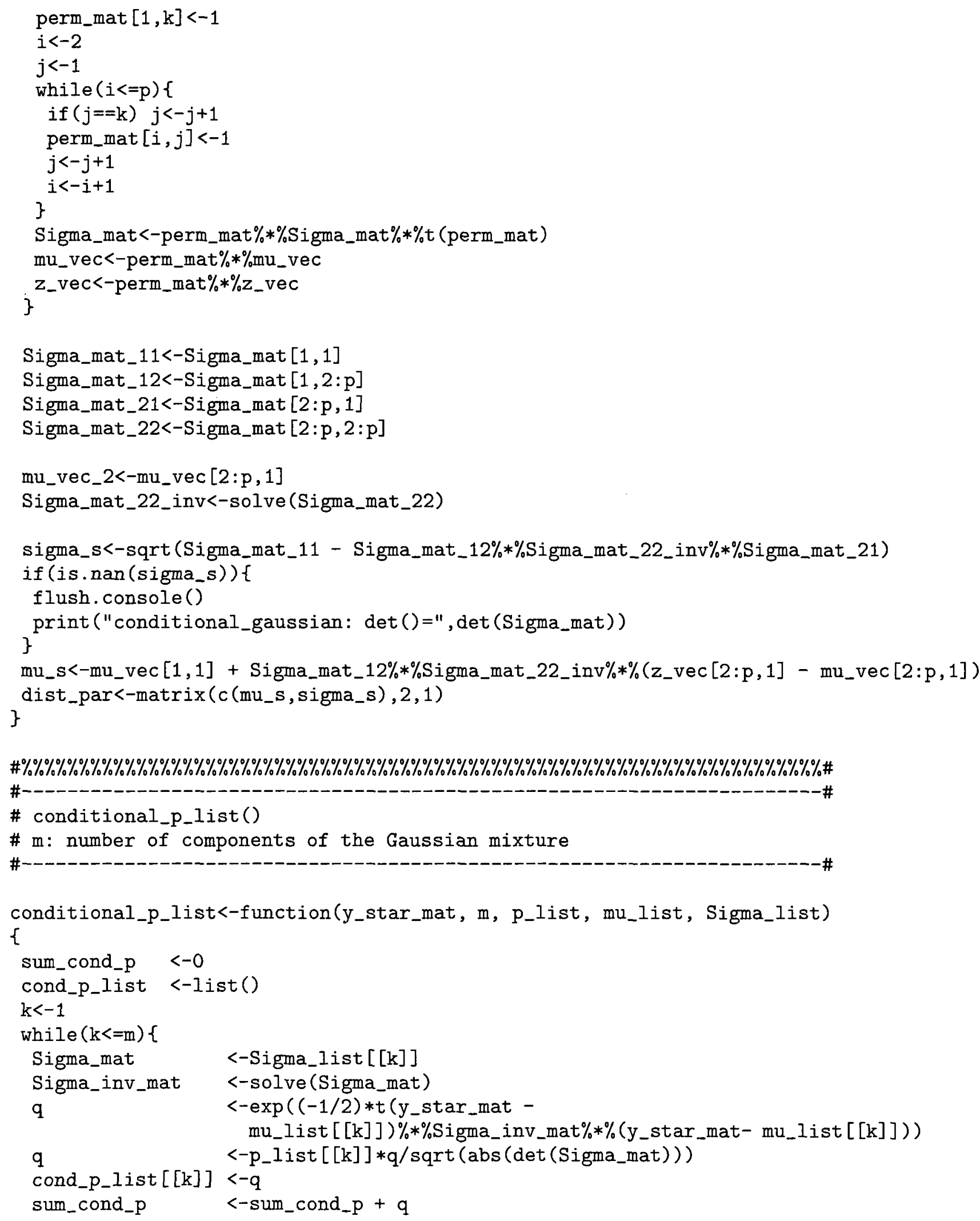




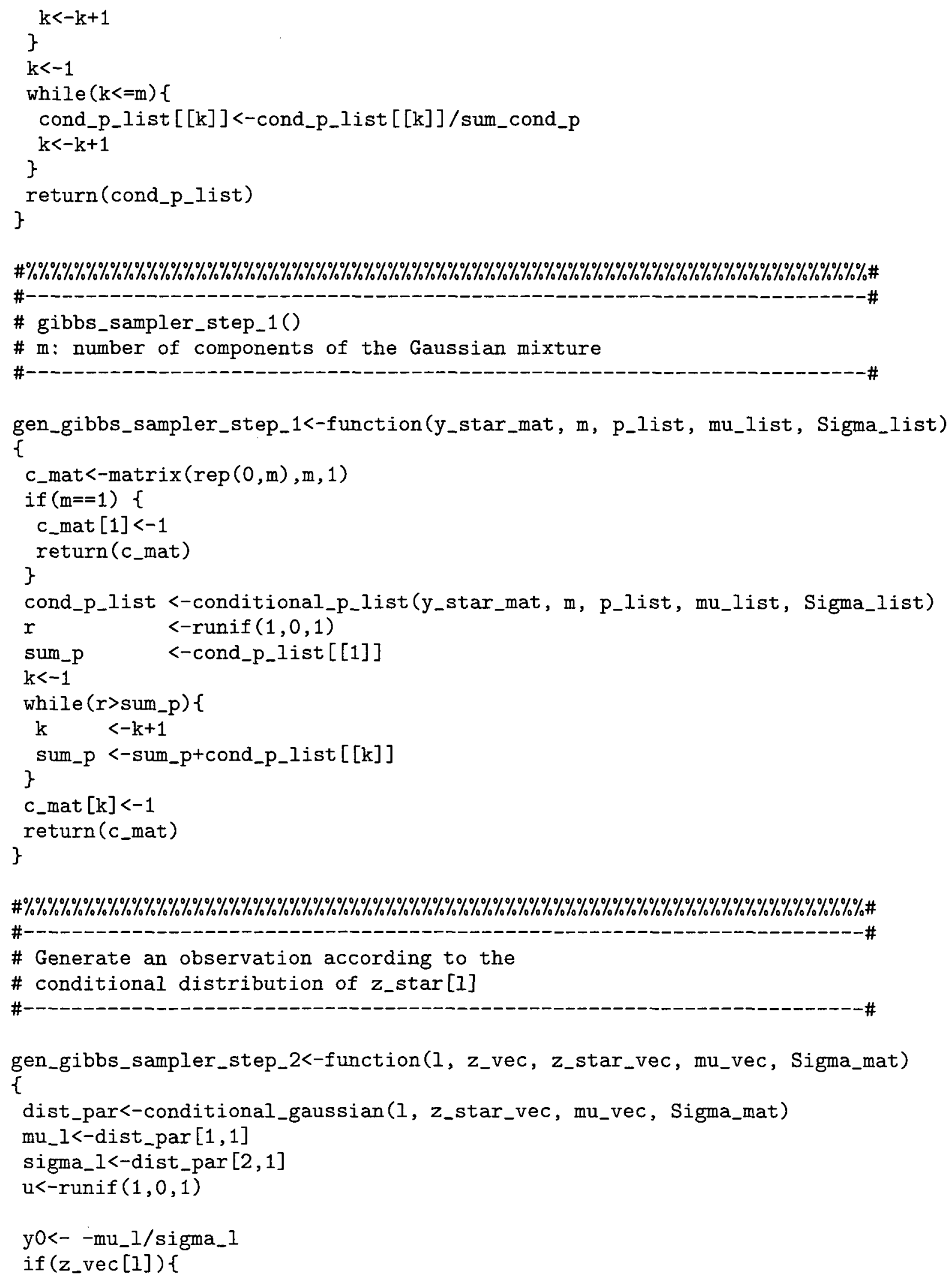



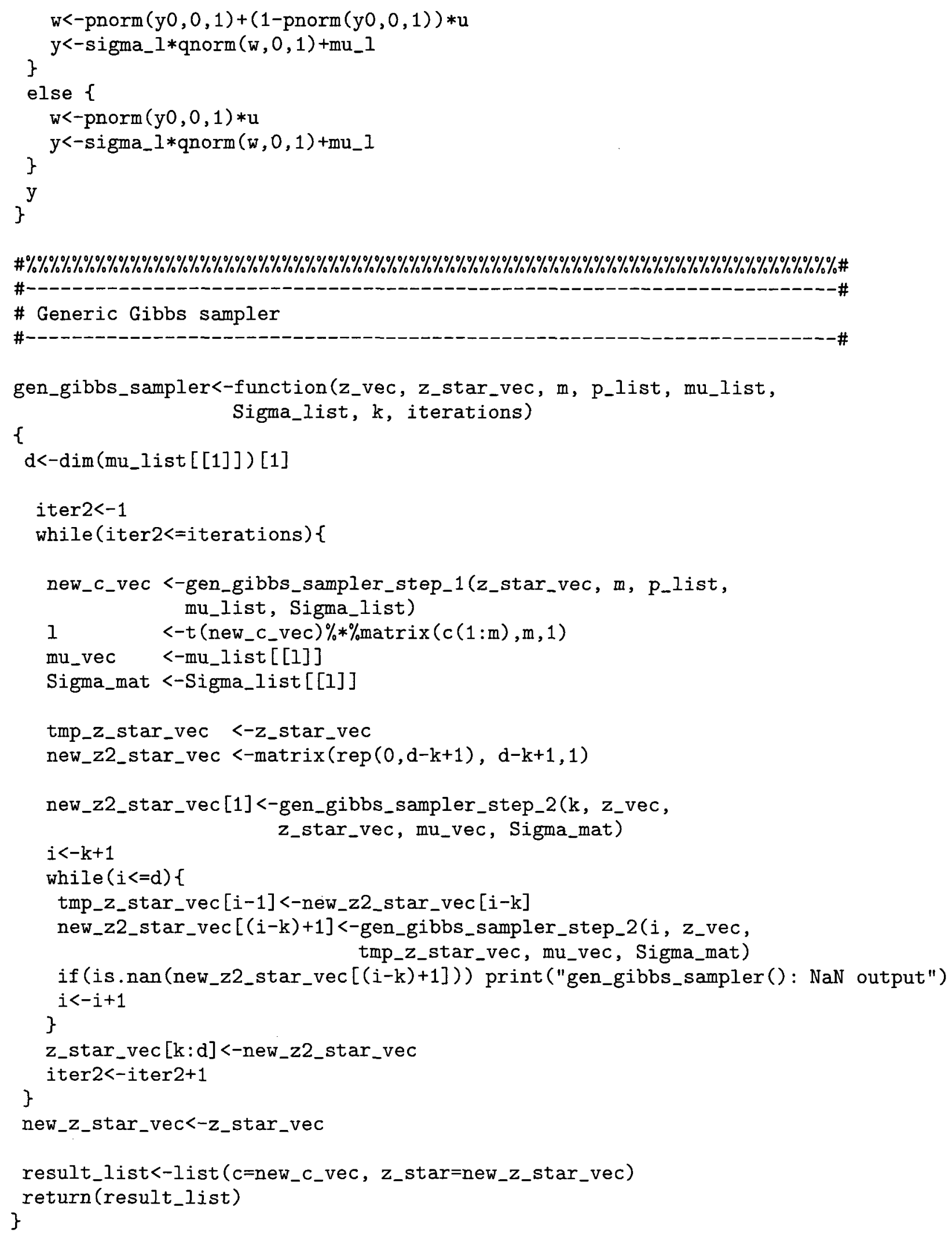

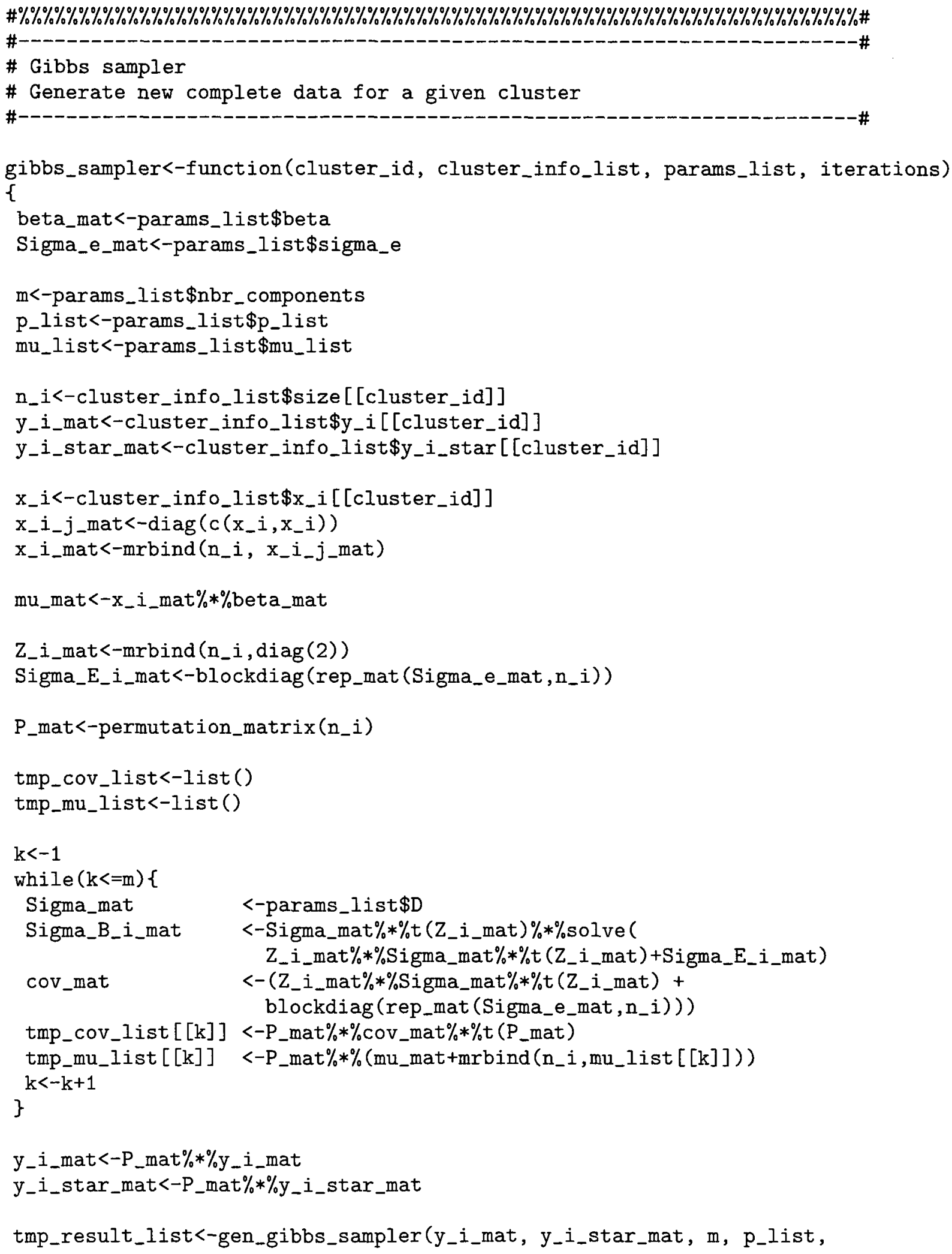
tmp_mu_list, tmp_cov_list, $n_{-} i+1$, iterations)

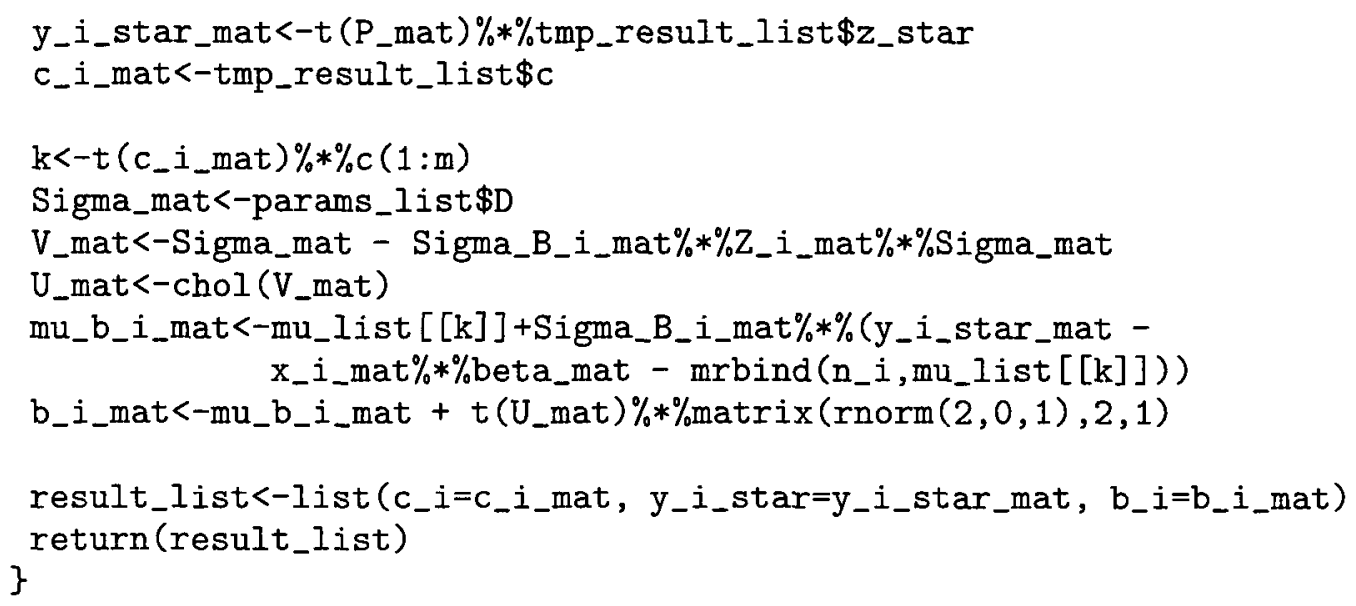




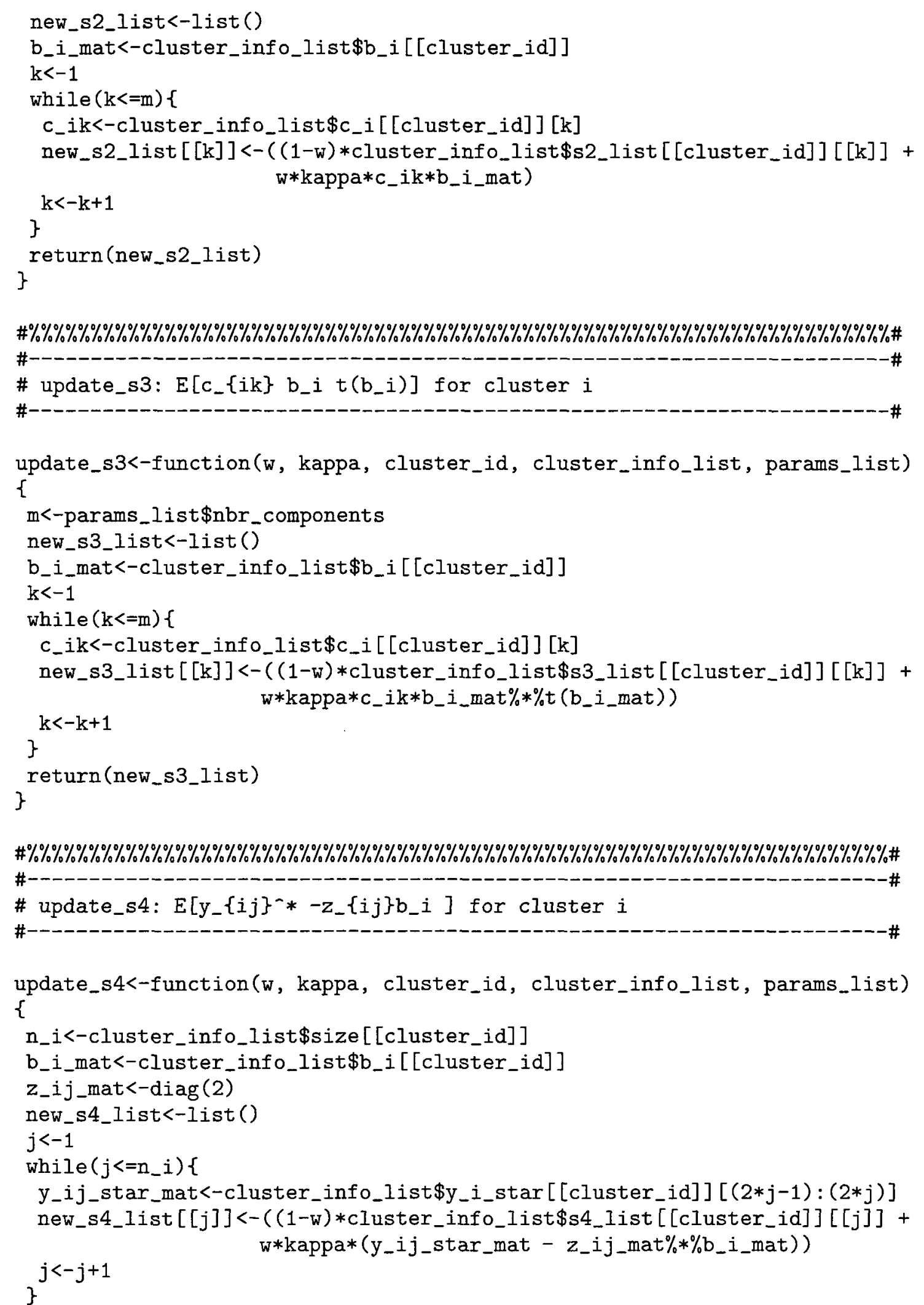




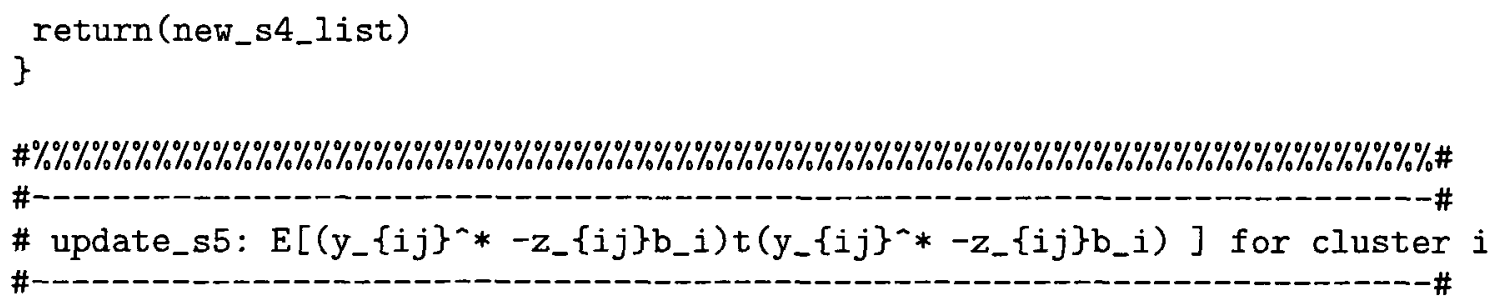




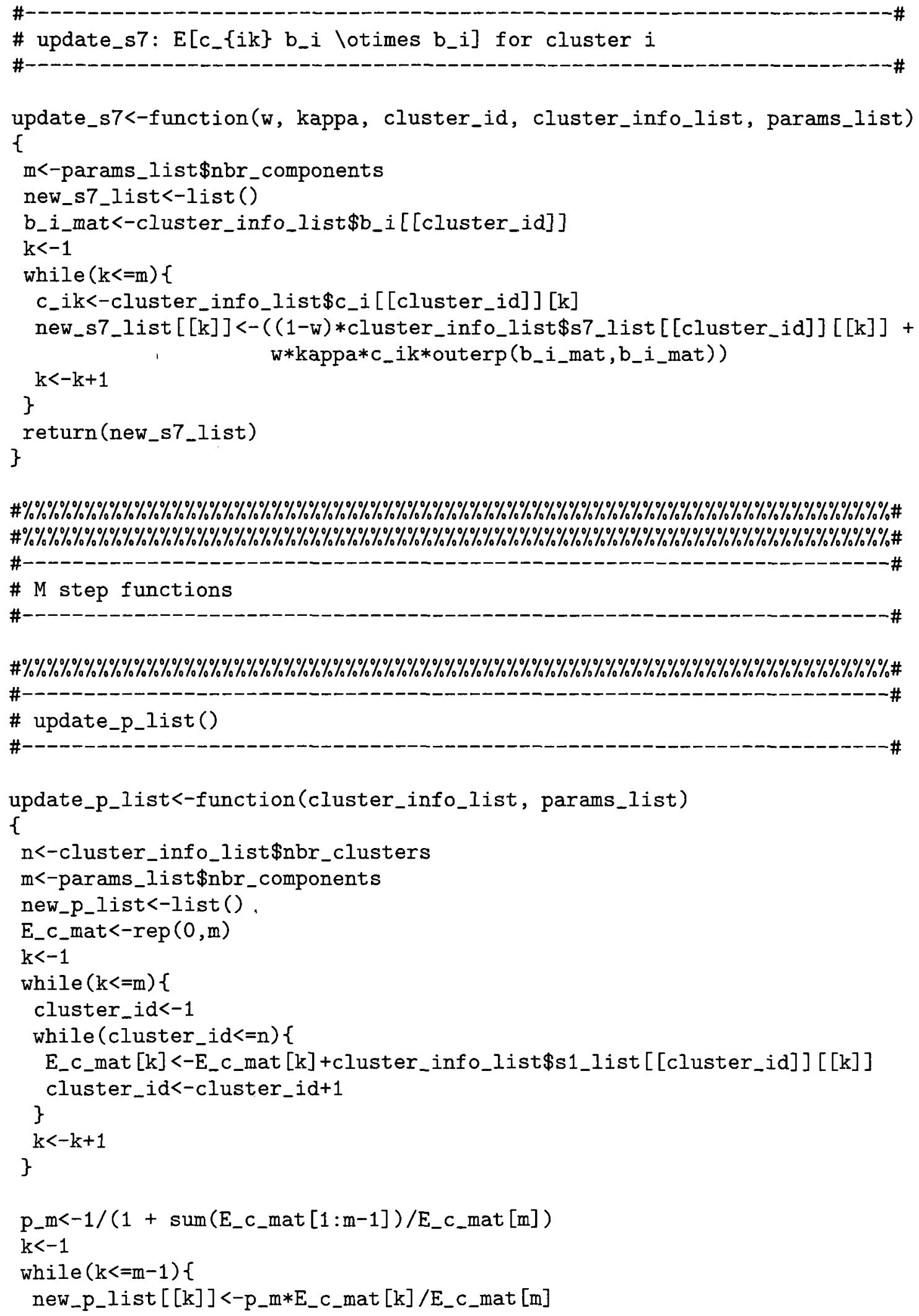




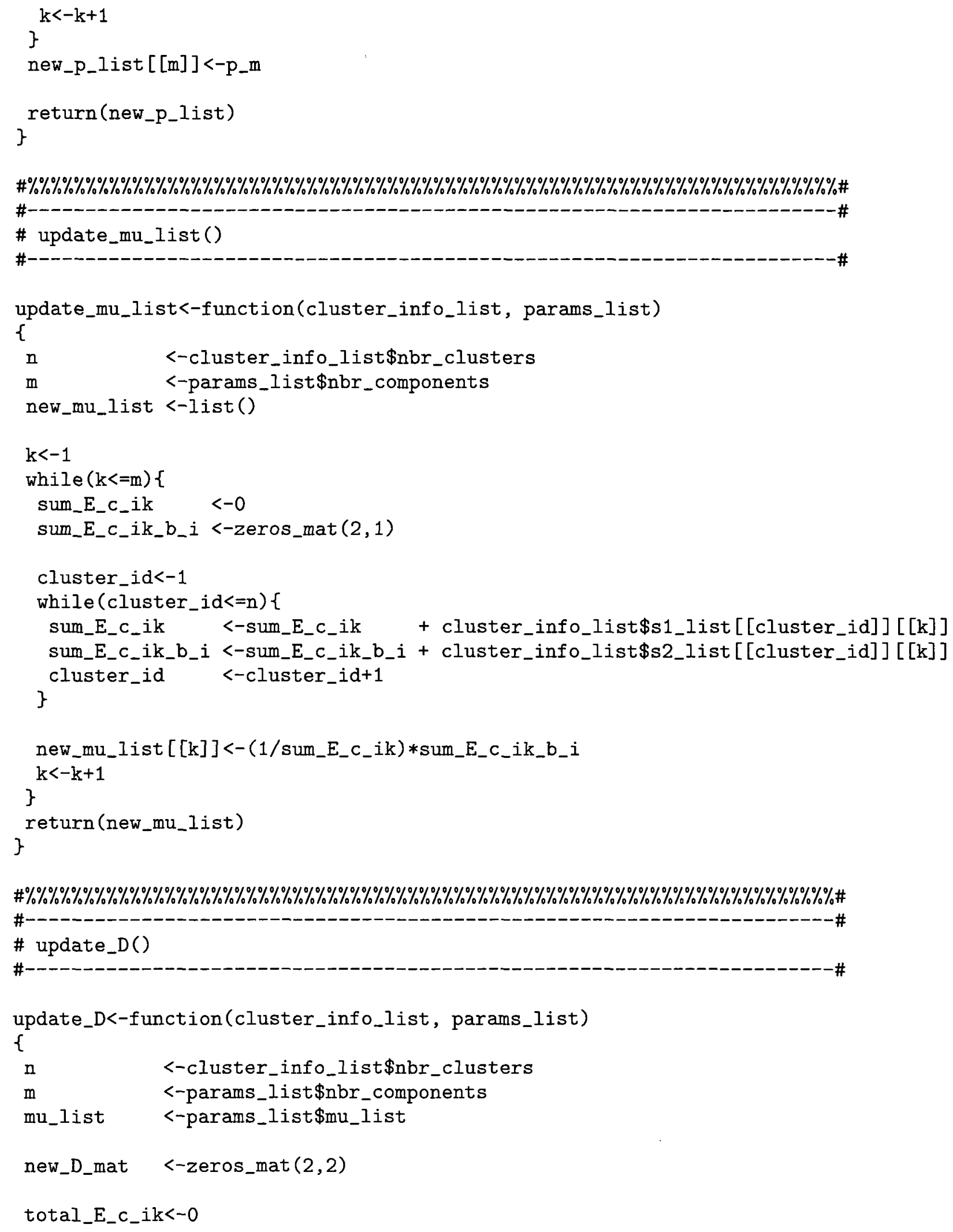




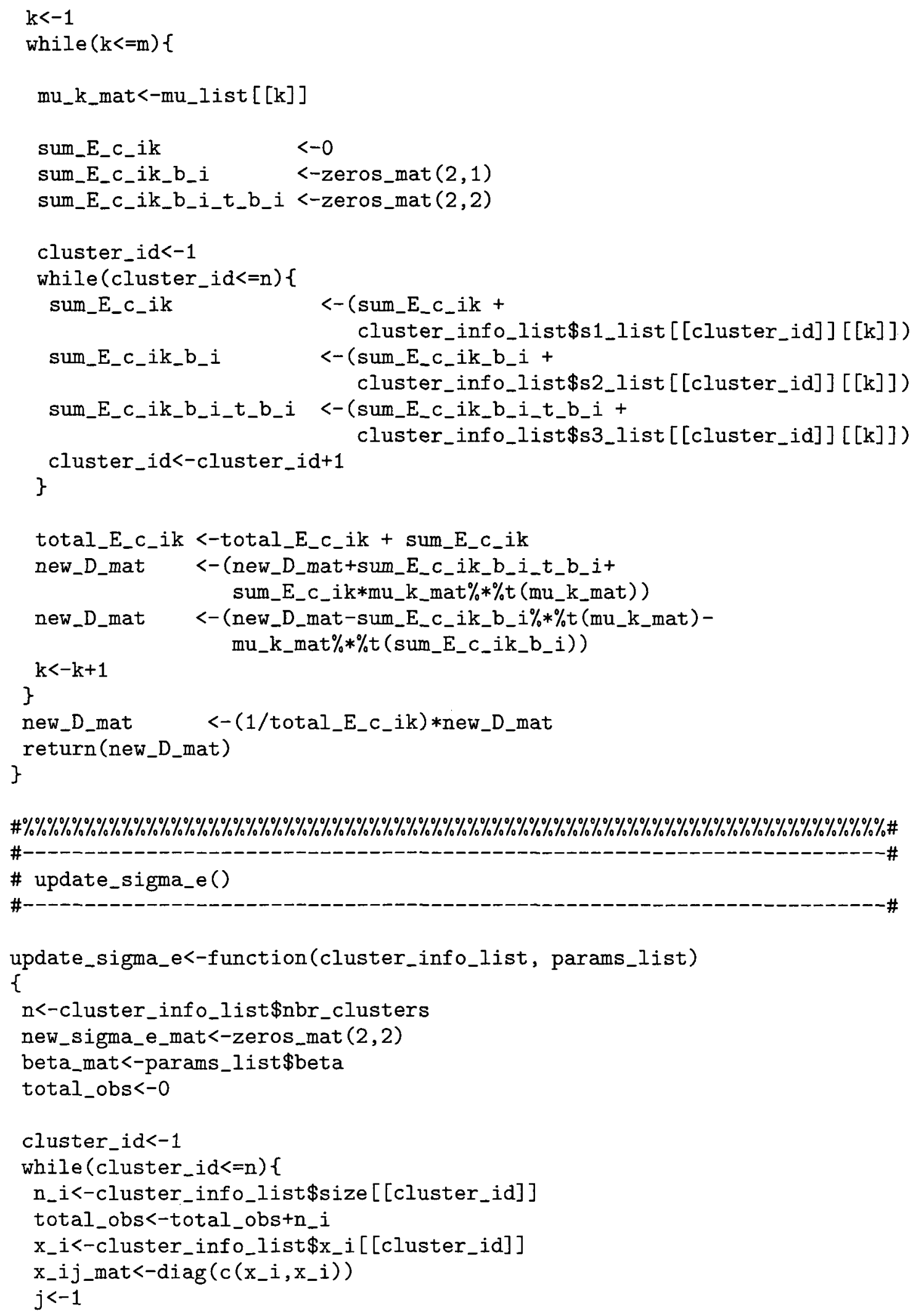




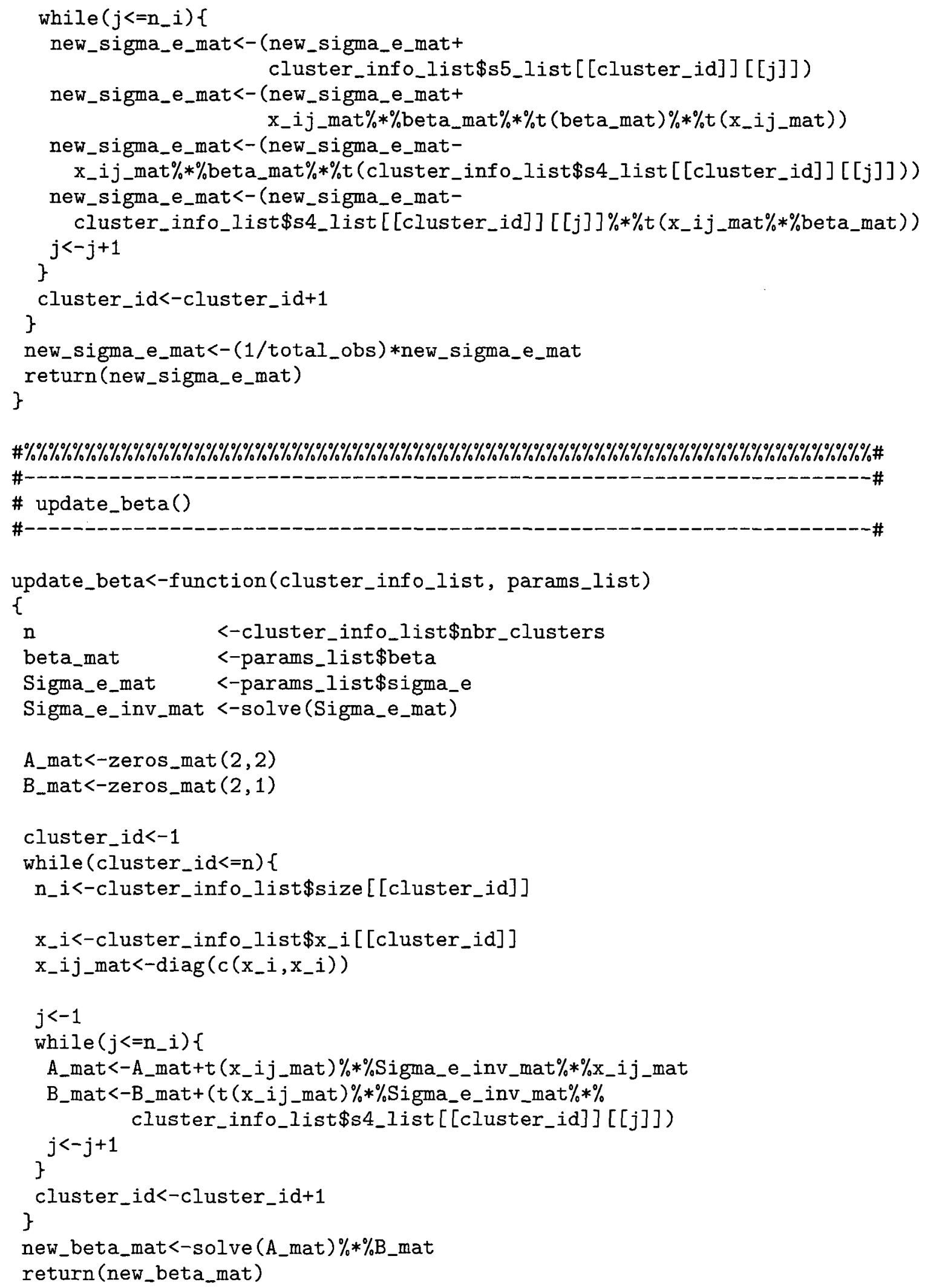



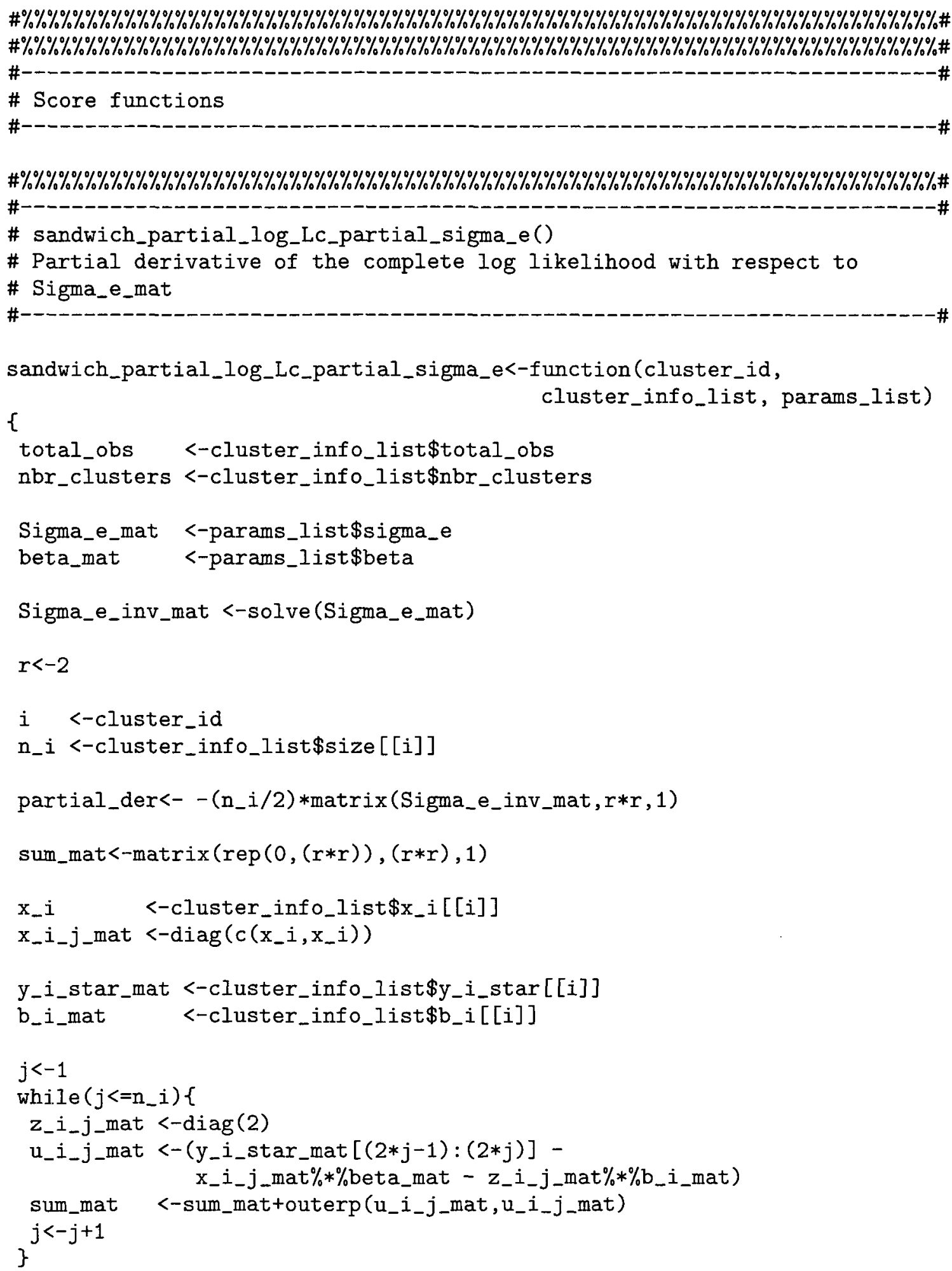


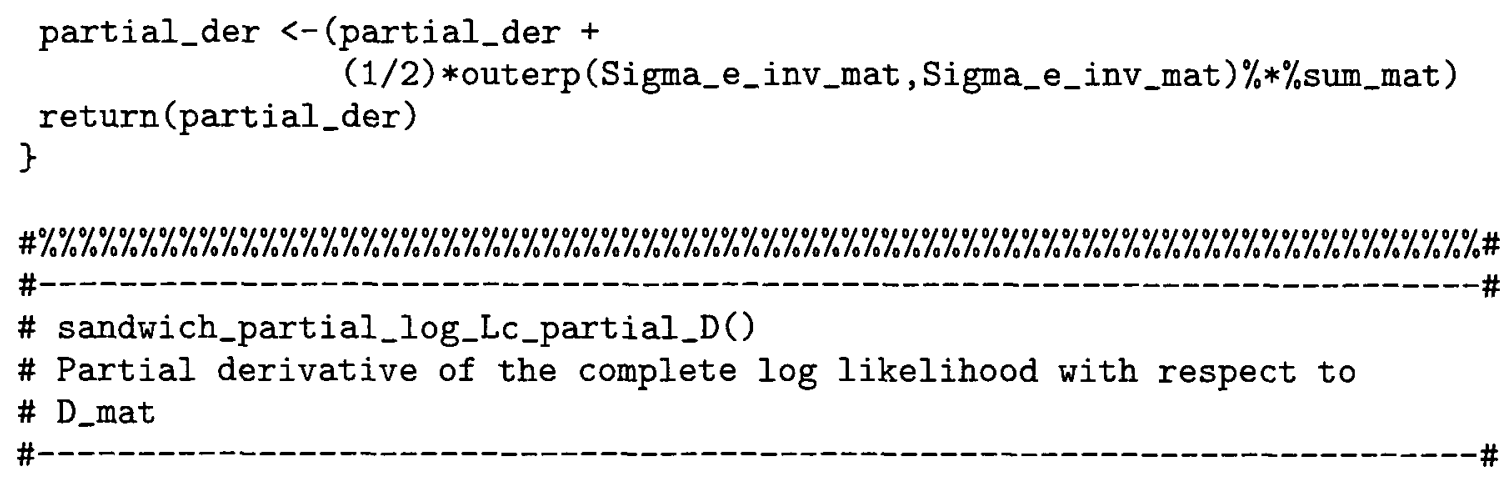




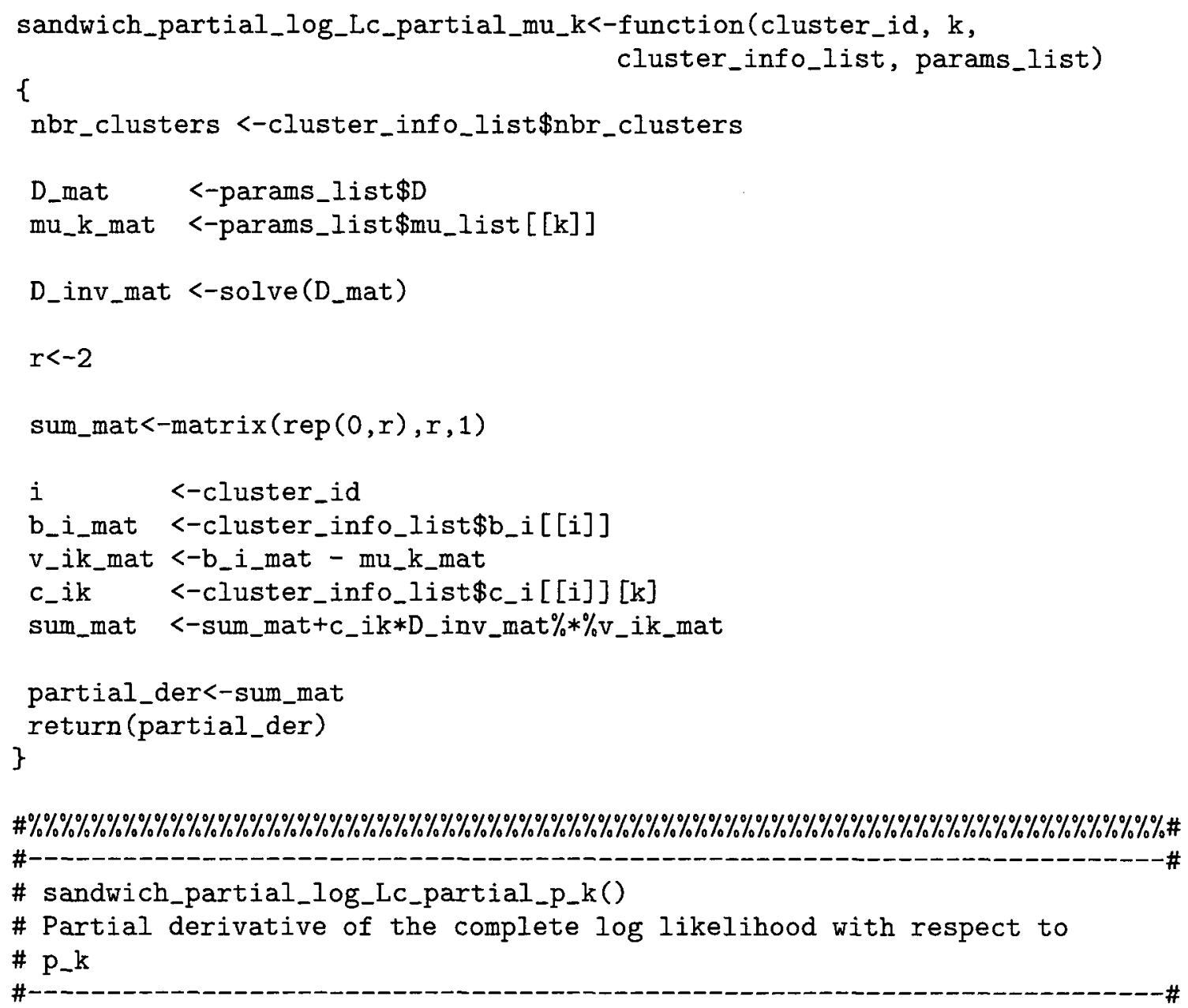




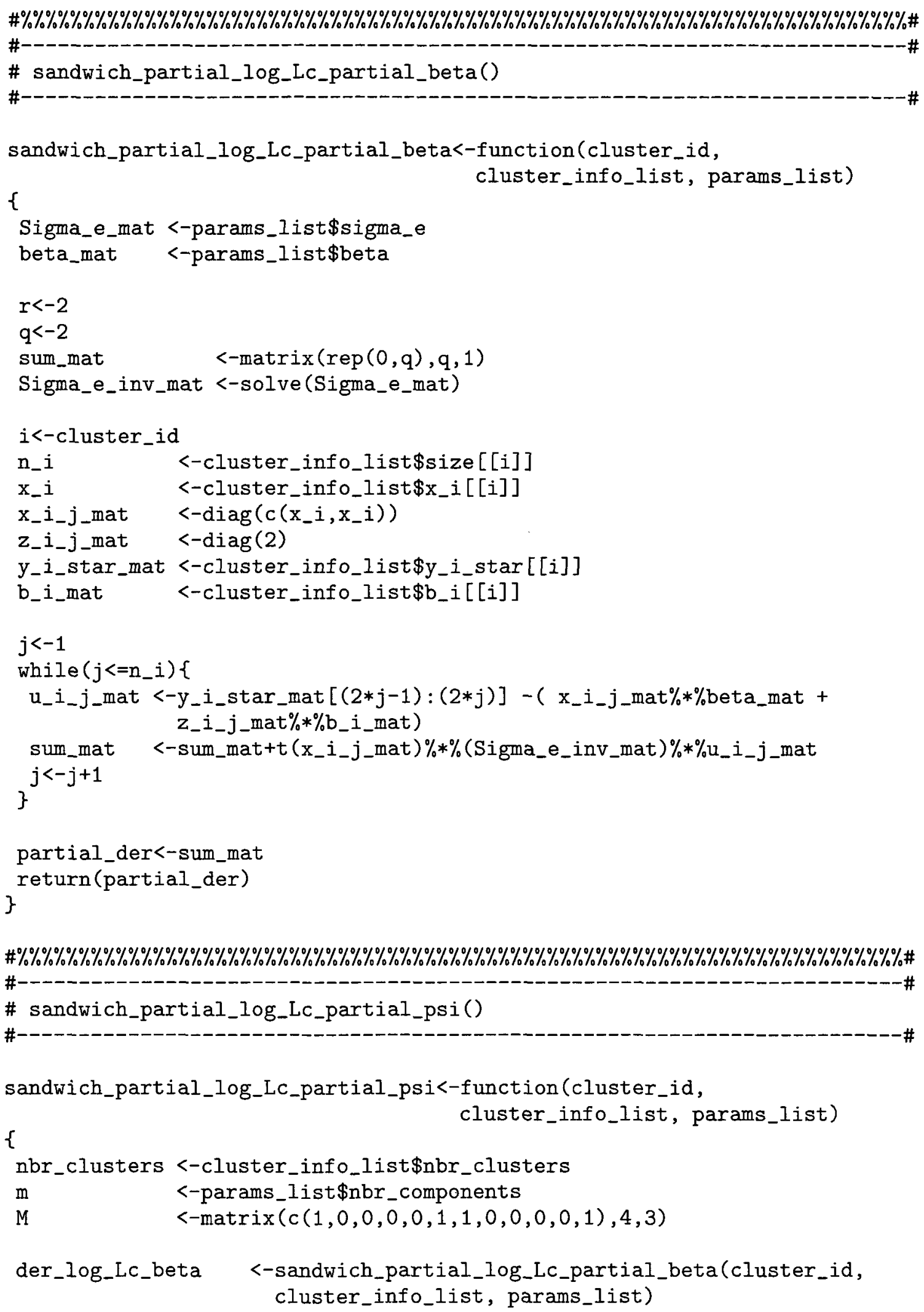




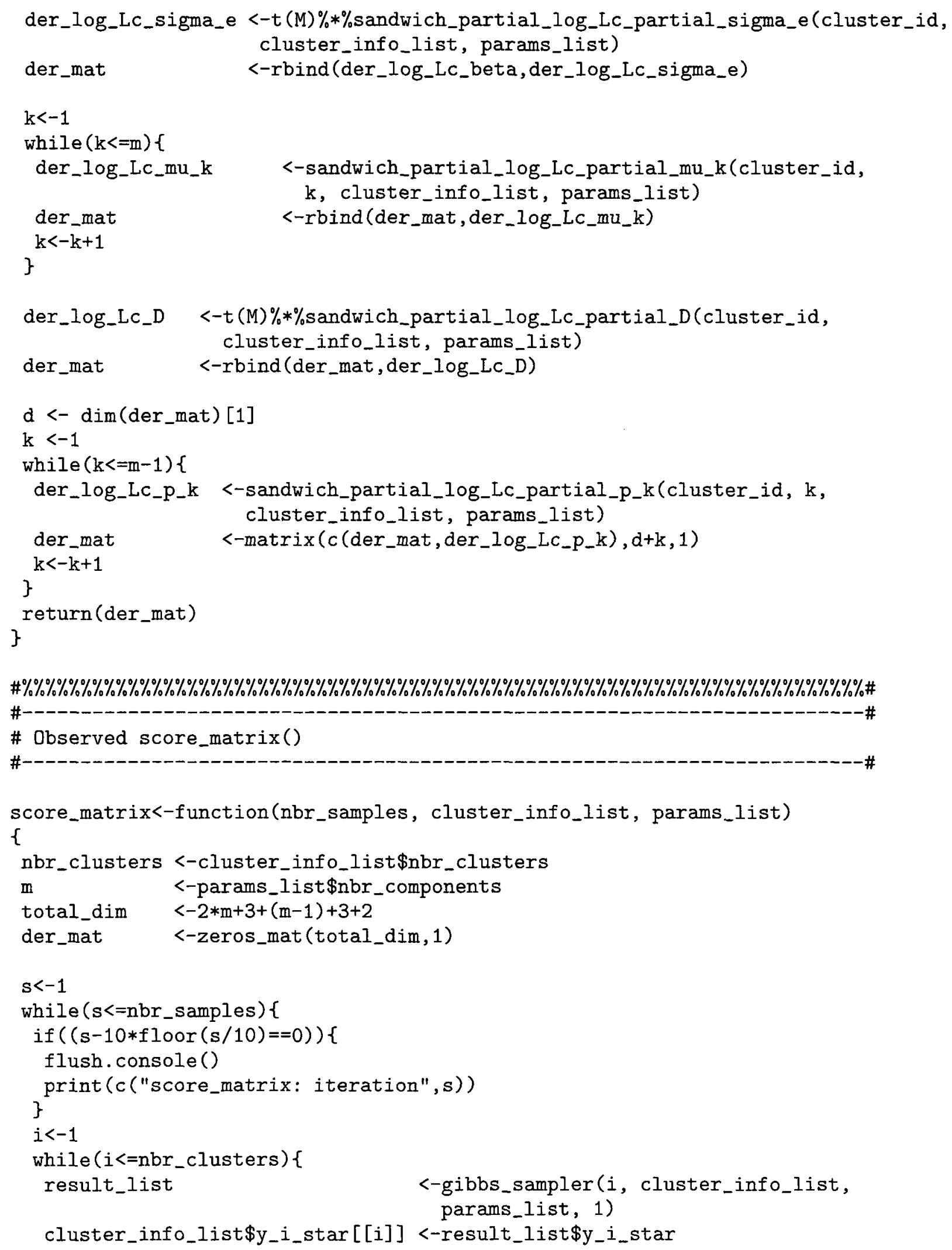




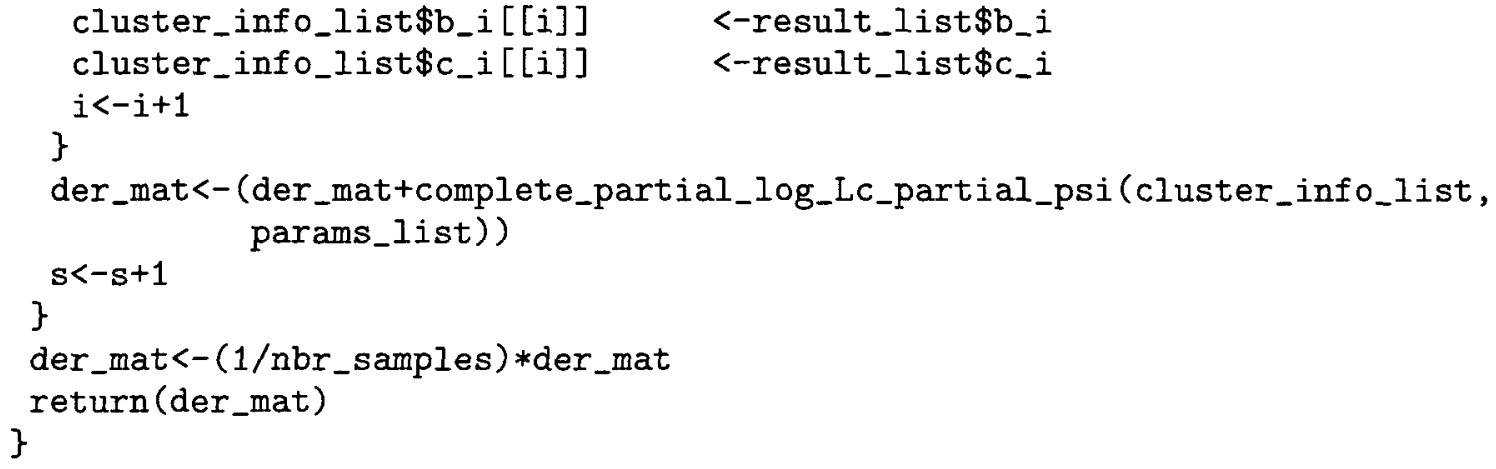




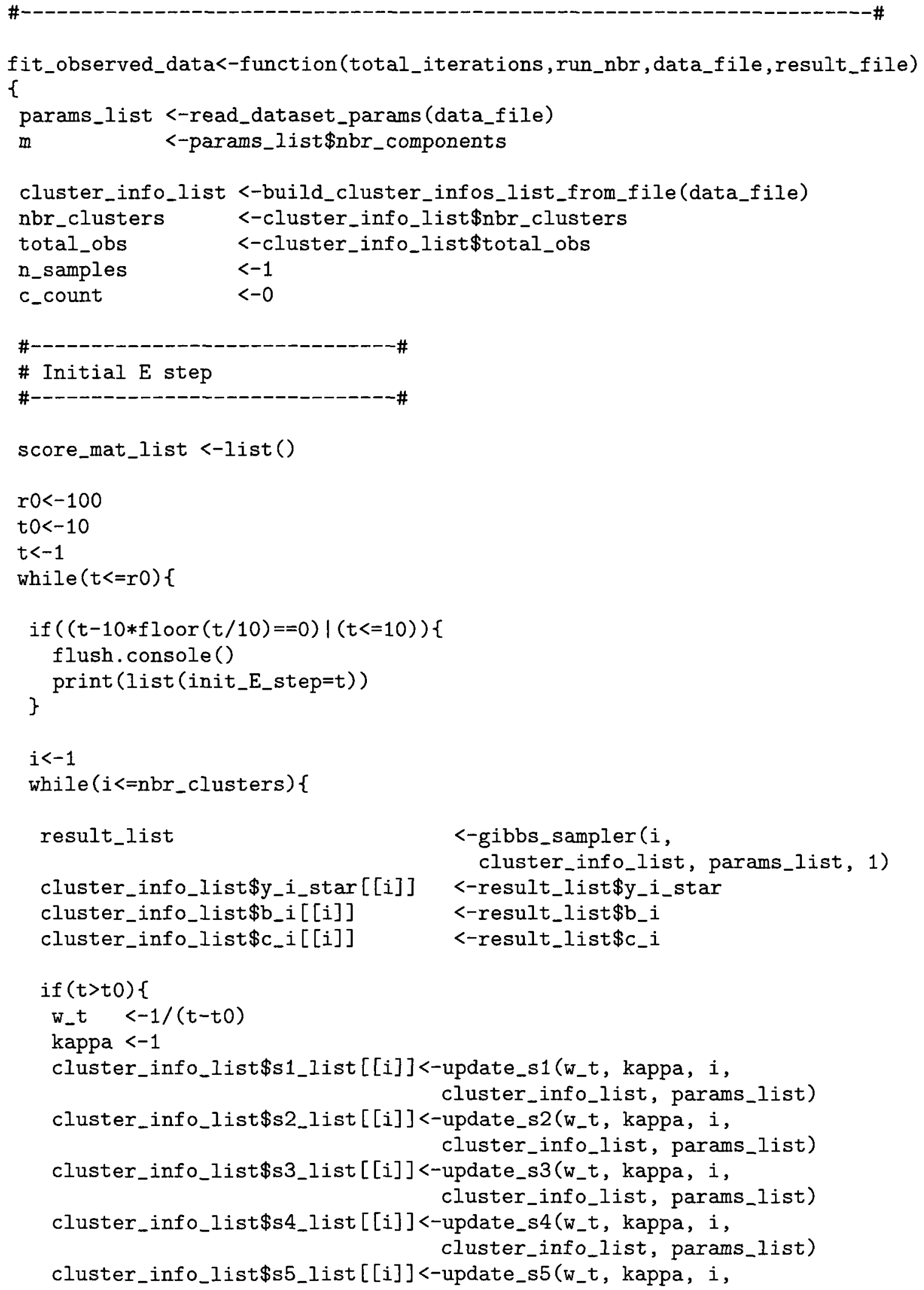




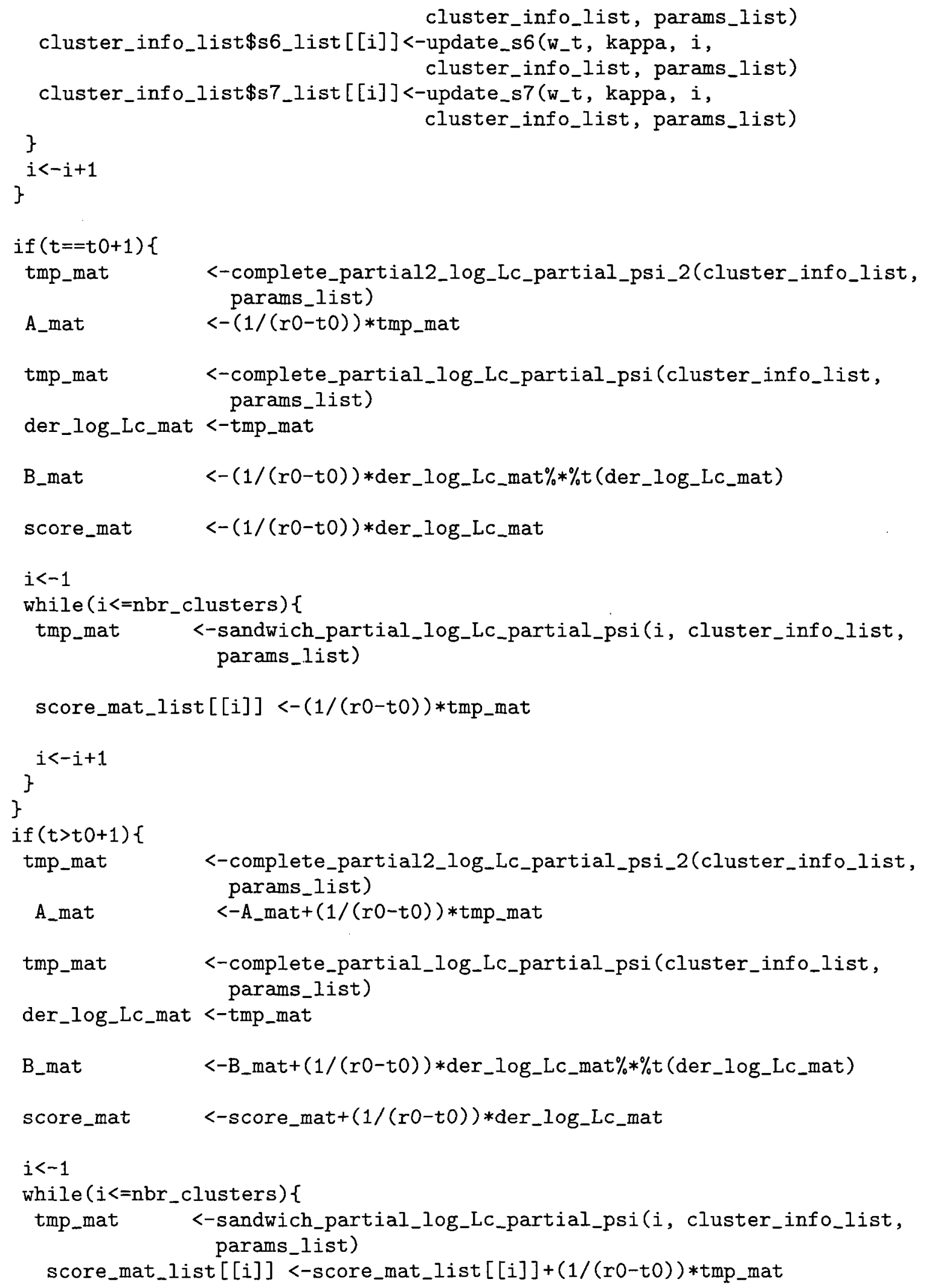




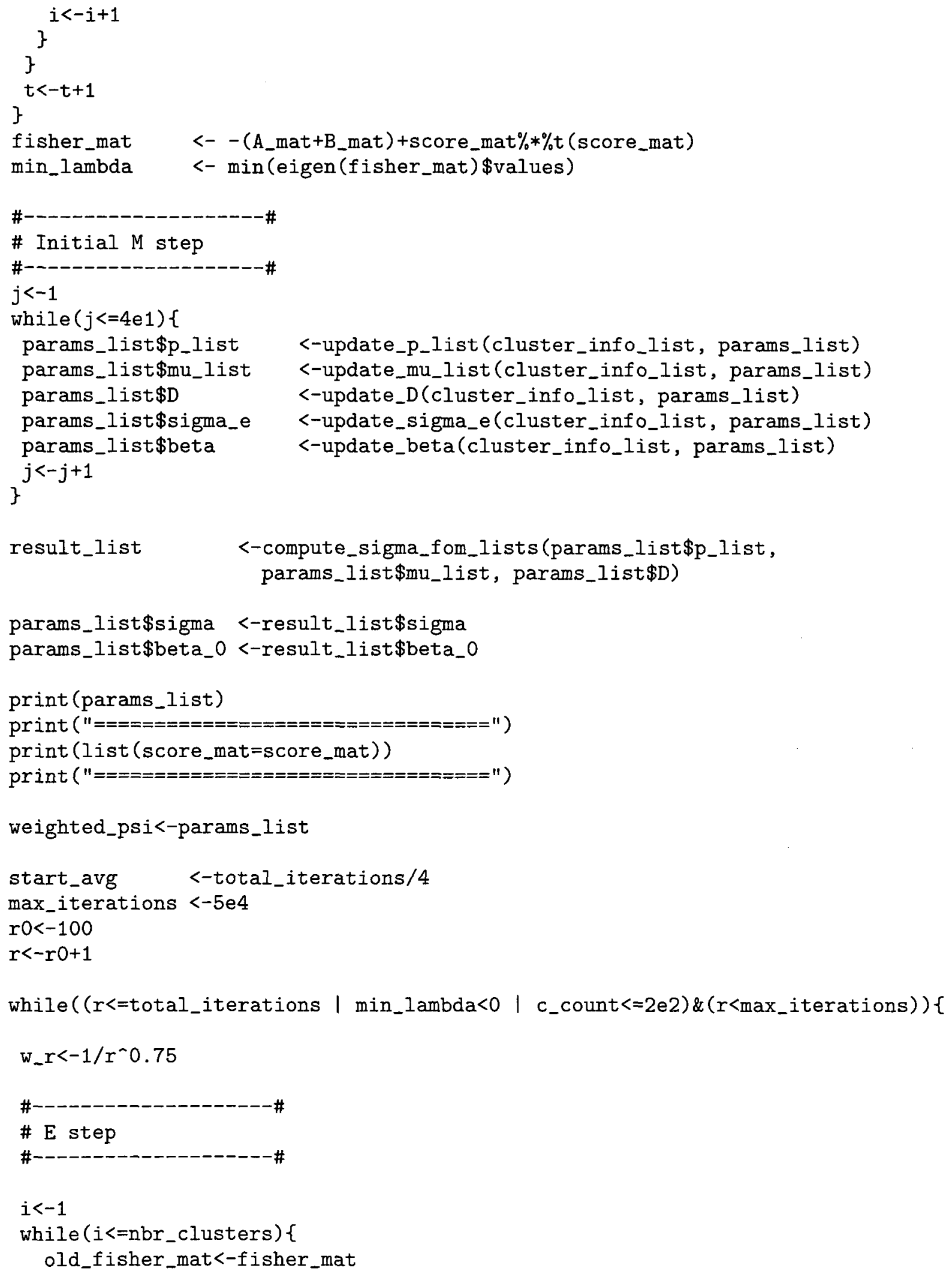




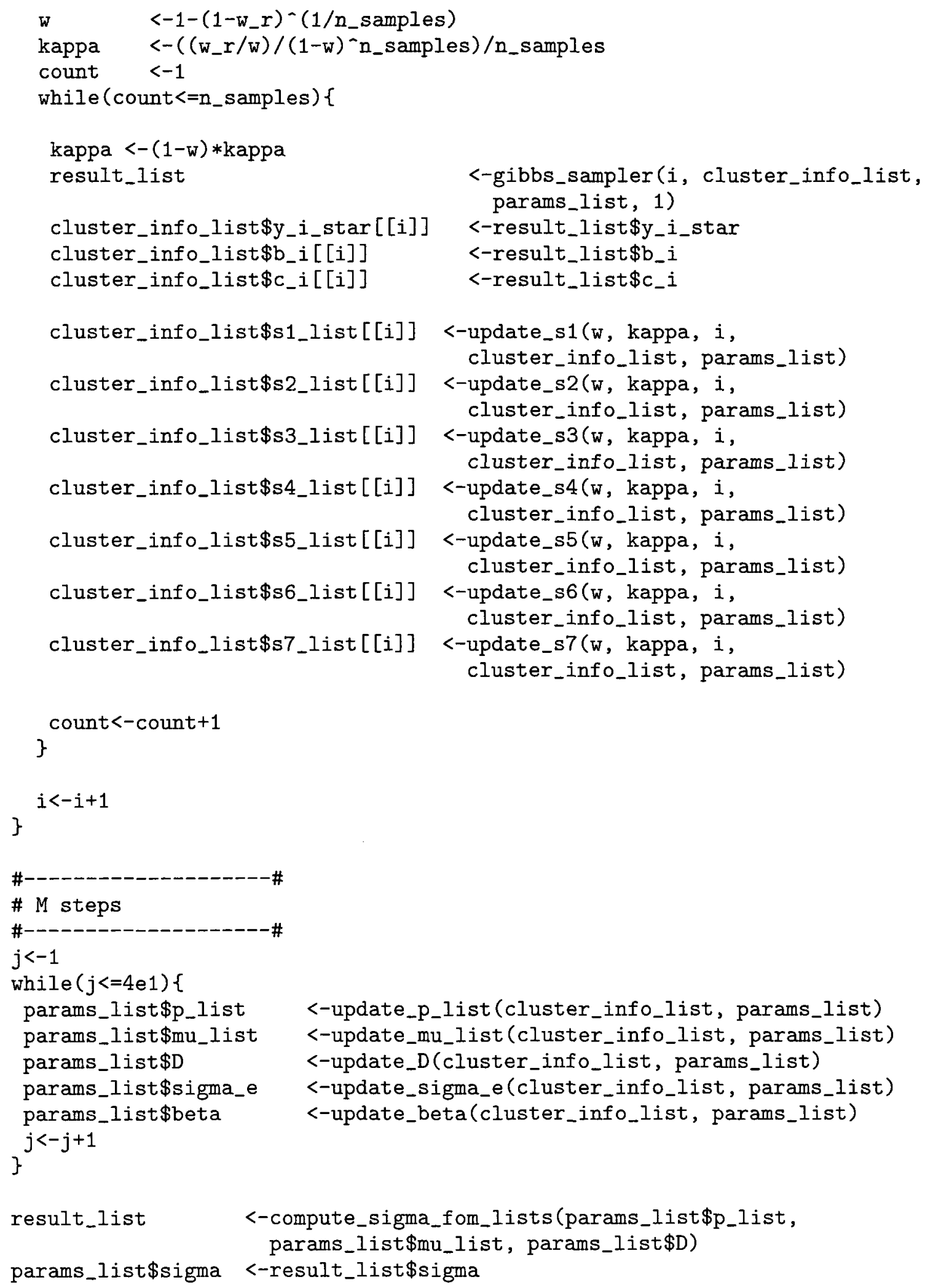




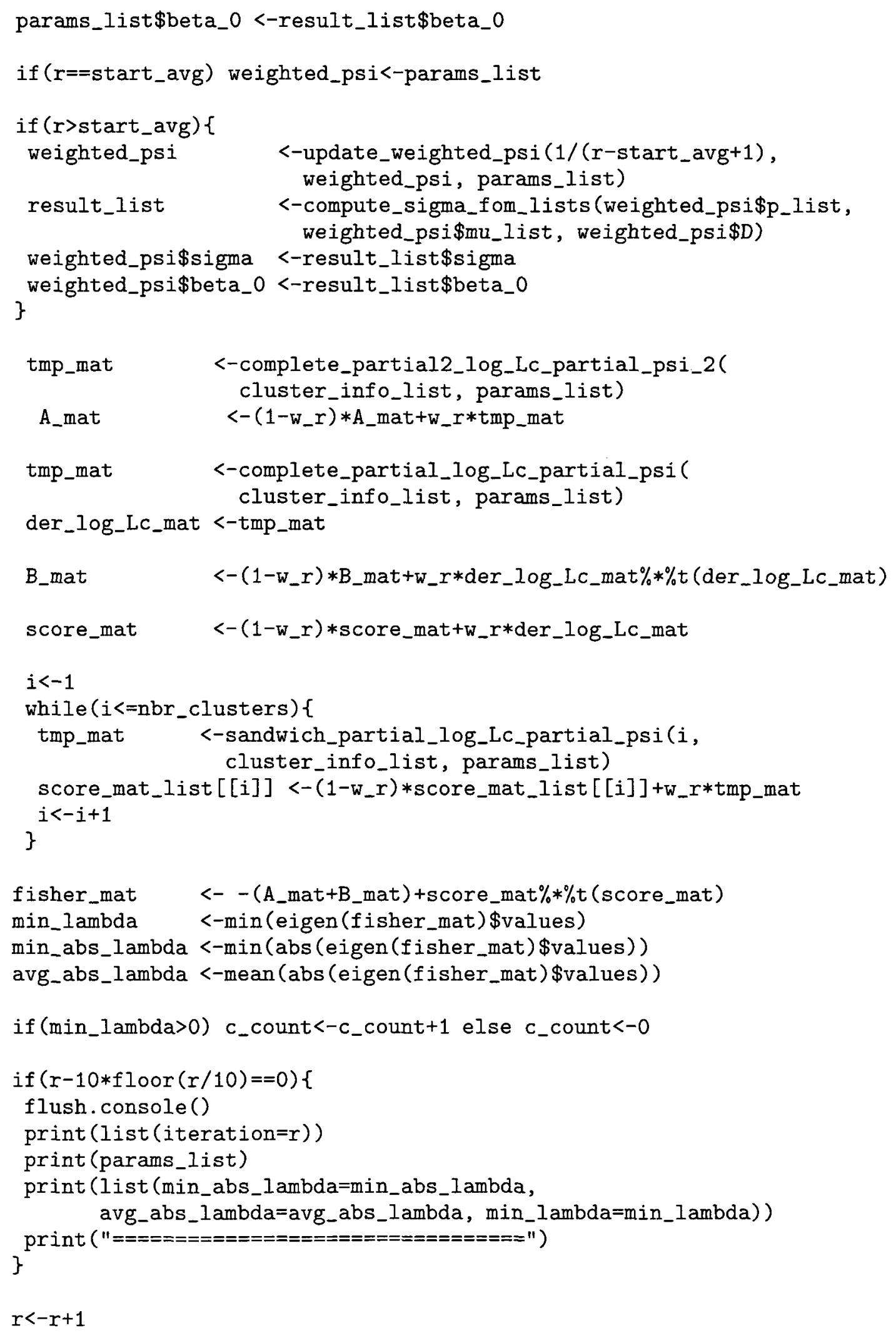




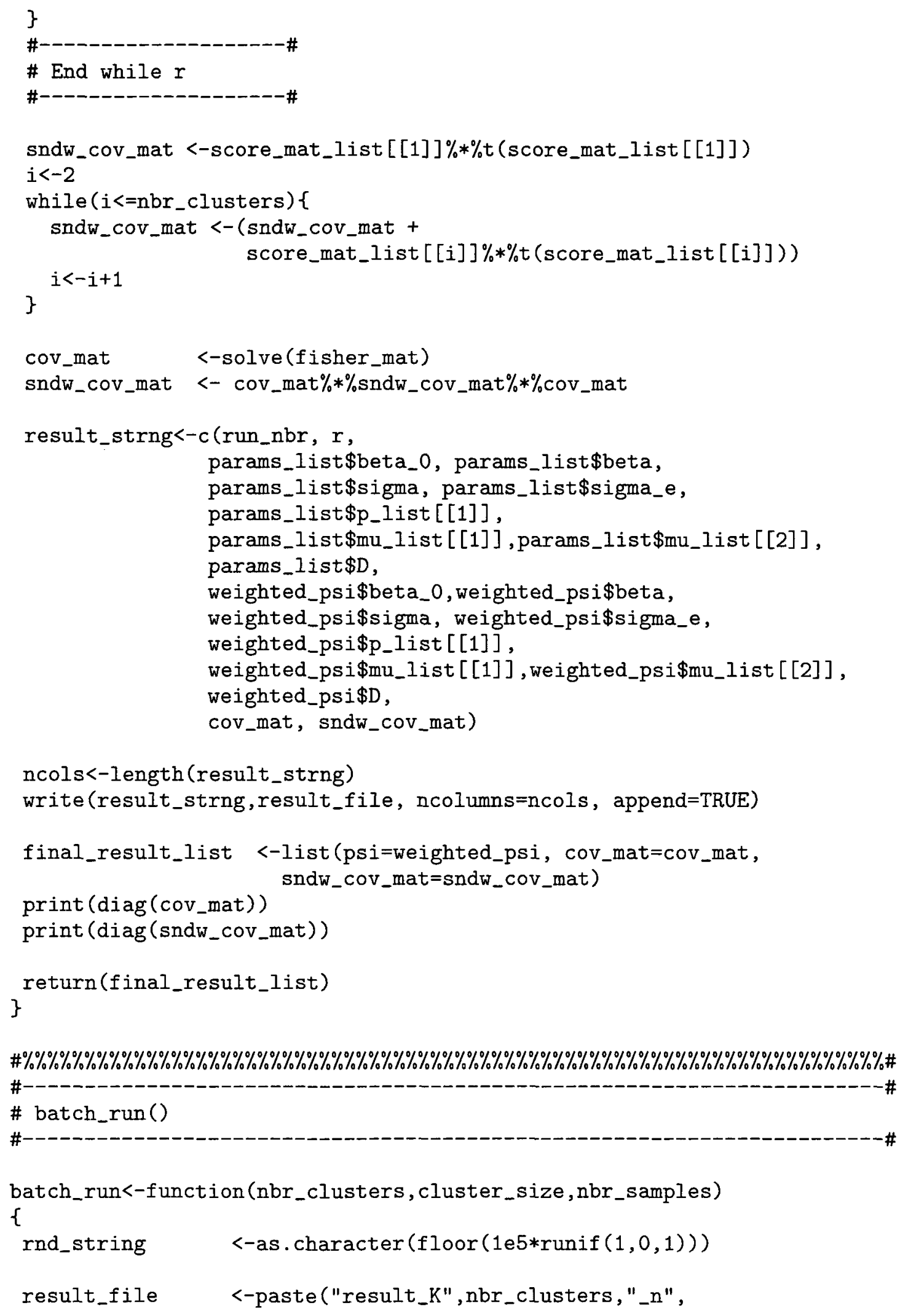




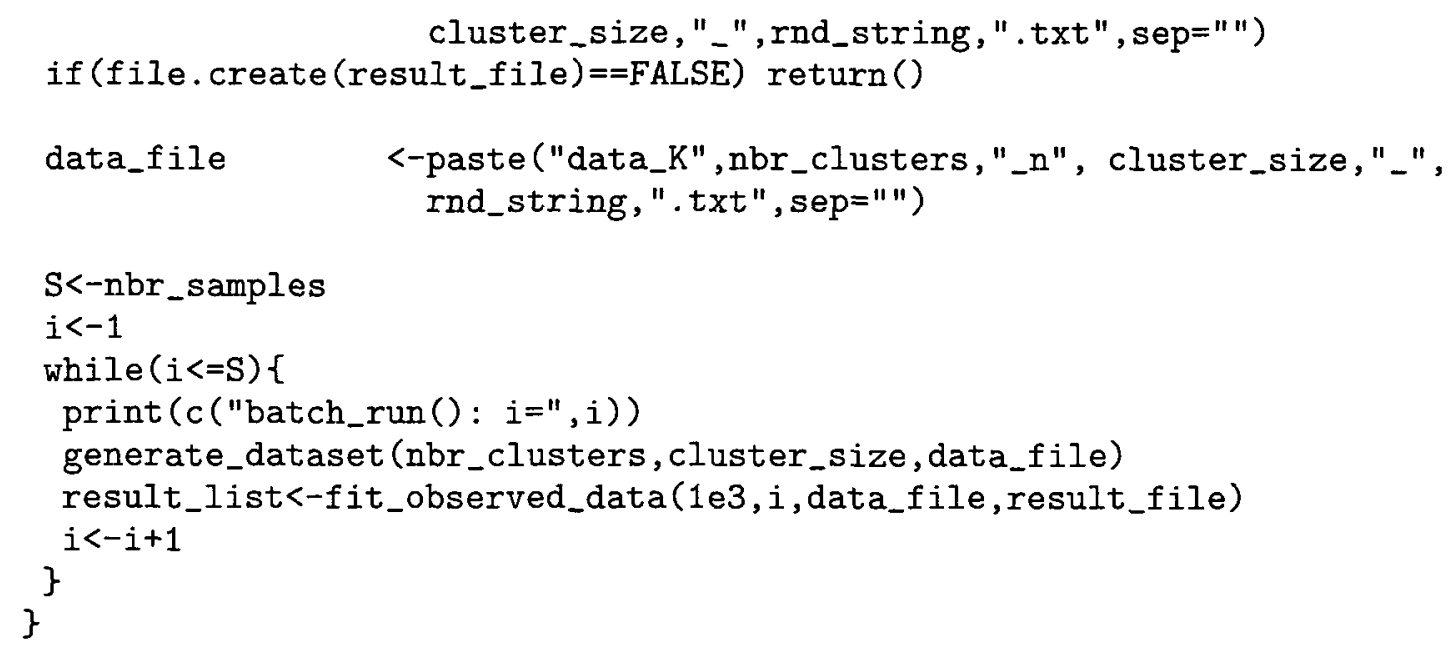

